\title{
PHYSICS OF LIMITING PHENOMENA IN SUPERCONDUCTING MICROWAVE RESONATORS: VORTEX DISSIPATION, ULTIMATE QUENCH AND QUALITY FACTOR DEGRADATION MECHANISMS
}

BY

\section{MATTIA CHECCHIN}

Submitted in partial fulfillment of the requirements for the degree of Doctor of Philosophy in Physics in the Graduate College of the Illinois Institute of Technology

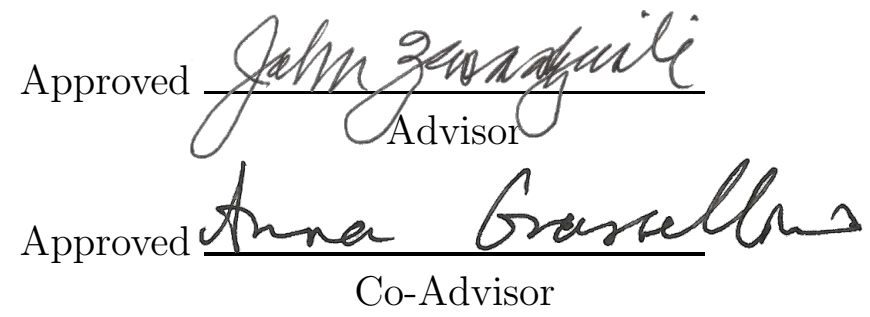

Chicago, Illinois

December 2016 
(c) Copyright by

MATTIA CHECCHIN

December 2016 


\section{ACKNOWLEDGMENT}

Firstly, I would like to express my sincere gratitude to my supervisor Dr. Anna Grassellino for her enormous support, guidance, motivation, inspiration and passion on the research. Without her as mentor, I would not be here writing this thesis and I would never had the possibility to perform high level research in the field I like the most. Thank you.

My sincere acknowledgment goes to my advisor Prof. John F. Zasadzinski. He always supported me during the whole Ph.D. and he always trusted and supported my research, giving me precious suggestions to understand the fundamental phenomena observed.

I would like to thank Dr. Alexander Romanenko for the continuous support of my Ph.D. study and related research, for his patience, deep knowledge and for bearing my long and boring disquisitions on the multiple topics described in this dissertation. His guidance helped me in all the time of research and writing of this thesis.

Besides my advisor and supervisors, I would like express my appreciation to Prof. Hasan Padamsee for his precious suggestions and comments, Prof. Yurii Shyl-nov for his essential support on the vortex resistance calculations, Prof. Alexander Gurevich for his important feedback on some of the work performed in this disserta-tion and Prof. Carlo U. Segre for his precious support during the initial stages of my Ph.D.

I want also to extend my deeply gratitude to the Technical Division Head Dr. Sergey A. Belomestnykh and the SRF Department Head Dr. Vyacheslav P. Yakovlev, and to the whole Cavity Performance and Testing group at the Fermi National Accelerator Laboratory, especially to: Dr. Sam Posen, Dr. Oleksandr Mel-

nychuk, Dr. Dmitri A. Sergatskov, Dr. Yulia Trenikhina, Dr. Sebastian Aderhold and 
Dr. Saravan Chandrasekaran, for their support and advises throughout the whole Ph.D. research.

A special thank you goes to all the new friends that I had the fortune to meet in this new experience in US. Antonio, Nicoló, Paolo, Alessio, Giuseppe, Giulia, Omar and all the others, day by day you made me feel at home.

My deepest and sincere gratitude goes to my partner Martina, she was always beside me during the whole journey that transformed us in what we are know. She gave me the strength to keep going and doing my best in life, work and study. I am fortunate that we grew up together.

Last but not the least, I would like to thank my parents and my sister Linda for supporting me throughout my entire academic path and my life in general. Without their support I would not had the courage to start my adventure in the US.

Thank you all. 
TABLE OF CONTENTS

Page

ACKNOWLEDGEMENT ....................... iii

LIST OF TABLES . . . . . . . . . . . . . . . . . . vii

LIST OF FIGURES . . . . . . . . . . . . . . . . . . . . . xiii

LIST OF SYMBOLS . . . . . . . . . . . . . . . . xiv

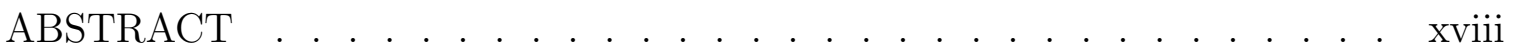

CHAPTER

1. INTRODUCTION . . . . . . . . . . . . . . . . 1

1.1. Why Superconducting Radio-Frequency Accelerators? . . . 1

1.2. Performance Limitation Impact on the Cost of Accelerators 3

1.3. Thesis Organization . . . . . . . . . . . . 5

2. ELEMENTS OF SUPERCONDUCTIVITY . . . . . . . . 7

2.1. Historical Overview . . . . . . . . . . . . . . . . . . . . . . 7

2.2. Ginzburg-Landau Theory . . . . . . . . . . . . . . . . . 12

2.3. Magnetic Flux Structures in Superconductors . . . . . . . 14

2.4. Magnetic Flux Dynamics . . . . . . . . . . . . . . 21

2.5. Mattis-Bardeen Surface Impedance . . . . . . . . . . . . 25

3. SUPERCONDUCTING ACCELERATING CAVITIES . . . . . 29

3.1. Accelerating Cavities . . . . . . . . . . . . . . . . . . . 29

3.2. Radio-Frequency Measurements . . . . . . . . . . . . . . 39

3.3. Superconducting Accelerating Cavities Performance . . . . 52

4. VORTEX SURFACE IMPEDANCE . . . . . . . . . . . 56

4.1. Chapter Overview . . . . . . . . . . . . . . . . 56

4.2. Single Vortex Resistivity . . . . . . . . . . . . . . 58

4.3. Multiple Vortices Surface Impedance . . . . . . . . . . 65

4.4. Model vs Experimental Data . . . . . . . . . . . . . 76

4.5. Summary . . . . . . . . . . . . . . . . . . . . . . . . . . 79

5. ACCELERATING GRADIENT LIMITATIONS . . . . . . . . 82

5.1. Chapter Overview . . . . . . . . . . . . . . . . . . . 82

5.2. Description of the Quench Phenomenon . . . . . . . . 83

5.3. Lower Critical Field Numerical Calculation . . . . . . . 87 
5.4. Superheating Field Numerical Calculation . . . . . . . . . 94

5.5. Experimental Data and Numerical Calculations . . . . . . 98

5.6. Summary . . . . . . . . . . . . . . . . 106

6. ENHANCEMENT OF THE ACCELERATING GRADIENT . . 108

6.1. Chapter Overview . . . . . . . . . . . . . . . . . 108

6.2. The Classic Bean Livingston Barrier . . . . . . . . . . . 109

6.3. The Dirty Layer Effect on Vortex Nucleation . . . . . . . 118

6.4. Vortex Penetration Delay . . . . . . . . . . . . . . . . . 128

6.5. Nitrogen Infusion for High $Q_{0}$ at High Gradients . . . . . . 131

6.6. Summary . . . . . . . . . . . . . . . . . 135

7. QUALITY FACTOR DEGRADATION DUE TO QUENCH . 137

7.1. Chapter Overview . . . . . . . . . . . . . . . . . 137

7.2. Experimental Set-up . . . . . . . . . . . . . . . . . . 138

7.3. Experimental Results . . . . . . . . . . . . . . . . . . . 140

7.4. Magnetic Field Redistribution During Quench . . . . . . . 146

7.5. Quality Factor Recovery Mechanism . . . . . . . . . . . . 149

7.6. Magnetic Flux Migration . . . . . . . . . . . . . . . . 151

7.7. Summary . . . . . . . . . . . . . . . . . 157

8. CONCLUSIONS . . . . . . . . . . . . . . . . . . . . . 159

8.1. Quality Factor Limitations due to Pinned Vortices . . . . . 159

8.2. Accelerating Gradient Limitation and Enhancement . . . . 160

8.3. Quality Factor Degradation by Quench . . . . . . . . . . 162

APPENDIX .......................... . . . 165

A. DIMENSIONLESS GINZBURG-LANDAU EQUATIONS . . . . 165

B. VORTEX SURFACE RESISTANCE NUMERICAL CODE . . . 168

C. SHOOTING METHOD . . . . . . . . . . . . . . . . . . . . 172

D. $H_{C 1}$ AND $H_{S H}$ NUMERICAL CODES . . . . . . . . . . . . . 175

D.1. Lower Critical Field Numerical Code . . . . . . . . . . . 176

D.2. Superheating Field Numerical Code . . . . . . . . . . . . 178

BIBLIOGRAPHY . . . . . . . . . . . . . . . . . . . . . . . . . . . . . 180 


\section{LIST OF TABLES}

Table

Page

4.1 Parameters used in the simulations for niobium. . . . . . . . . . . 68

4.2 Pinning point positions of the green points in Figure 4.5. For all the points $U_{0}=1.1 \mathrm{MeV} / \mathrm{m}$ and $l=70 \mathrm{~nm}$. . . . . . . . . . . . . . .

5.1 Cavities studied. The values $\sigma_{B_{p}}, \sigma_{l}$ and $\sigma_{\kappa}$ are the errors associated to $B_{p}, l$ and $\kappa$ respectively. . . . . . . . . . . . . . . . . . . .

7.1 Cavities studied with respective thermal treatments and quench fields. Doped cavities were treated with 25 mTorr of $\mathrm{N}_{2}$ and with a post treatment chemistry $(\mathrm{EP})$ of $5 \mu \mathrm{m}$. . . . . . . . . . . . . . . . 


\section{LIST OF FIGURES}

Figure

Page

1.1 Pie diagram of the percentage contributions to the cost of the ILC. Data reported in [1]. Acronyms meaning: cryomodules (CM), high

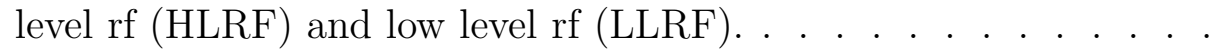

2.1 Variation of the free energy density as a function of the order parameter for normal-conducting phase $\left(T>T_{c}\right)$, superconductiong phase $\left(T<T_{c}\right)$ and at transition $\left(T=T_{c}\right)$. . . . . . . . . . . .

2.2 In (a) the thermodynamic critical field is plotted as a function of the temperature, while in (b) an example of intermediate state observed by bitter decoration in a single crystal lead foil [2]. . . . . . . . .

2.3 In (a) dependence of the lower and upper critical fields with temperature. On the right, some examples of Mixed state are reported, where the vortex lattice was imaged by (b) bitter decoration of PbIn [3], (c) scanning tunnel microscopy of $\mathrm{NbSe}_{2}$ [4], (d) scanning tunnel microscopy of $\mathrm{MgB}_{2}$ [5] and (e) magneto-optical imaging of $\mathrm{NbSe}_{2}[6]$. . . . . . . . . . . . . . . . . . . . . . . . . . . .

2.4 In (a) a schematic representation of a vortex, while in (b) a sketch of the order parameter variation as a function of the radius in the local and non-local model.

2.5 Density of states of a superconductor and Fermi-Dirac distribution as a function of the energy relative to the Fermi level. . . . . . . 26

2.6 Surface resistance as a function of $T$ for $\mathrm{Pb}[7] \ldots$. . . . . . . . .

3.1 A $1.3 \mathrm{GHz}$ TESLA-type nine-cells cavity [8]. . . . . . . . . . . .

3.2 In (a) a single- and a multi-cells HEPL "pillbox" cavity [9] (the pictures are courtesy of Prof. H. Padamsee) . In (b) a schematic representation of a "pillbox" cavity of radius $a$ and length $h$ without beam pipes and of the system of coordinates used in the calculation.

3.3 Polar and axial plots of the axial electric field and transverse magnetic field for the mode $\mathrm{TM}_{010}$. Red correspond the high values of $E$ or $B$, while blue to zero. . . . . . . . . . . . . . . . . . . .

3.4 Electric and magnetic field simulations for a TESLA type elliptical cavity. . . . . . . . . . . . . . . . . . . . . 
3.5 Examples of reflected power signal as a function of time in conditions of under, critically and over coupling. The forward power pulse is shown in blue. . . . . . . . . . . . . . . . . . . . . . . . . . .

3.6 In (a) a simplified schematic of the rf system is reported, while in (b), (c) and (d) the top plate of the Dewar "VTS1", the vertical test stand and the rf measurement system at the Fermilab vertical test facility respectively are shown. . . . . . . . . . . . . . . . . .

3.7 Schematics of the cables and connections needed to perform the $\mathrm{rf}$ system calibration. . . . . . . . . . . . . . . . . . . . . . . .

3.8 In (a) TESLA type cavity equipped with T-map and Helmholtz coils, in (b) T-map board in detail and in (c) TESLA type cavity equipped with thermometers, fluxgates and Helmholtz coils. . . .

3.9 Example of cavities performance for different surface finishing. . .

4.1 In (a) tri-dimensional representation of the pinning potential considered. In (b) contour plot of two pinning potential acting on the fame flux line. . . . . . . . . . . . . . . . . . . . . . . .

4.2 Real part of the surface impedance as a function of the mean free path. The dashed-dotted lines correspond to the pinning and flux flow regimes, for small and large mean free path values respectively. In the inset the imaginary part of surface impedance is plotted as a function of the mean free path. . . . . . . . . . . . . . . . . .

4.3 Real part of the surface impedance as a function of the mean free path for different values of $U_{0}$. . . . . . . . . . . . . . . . . .

4.4 Simulation of the average power per unit of volume normalized to the current density squared for $l=10 \mathrm{~nm}$ and $U_{0}=1.1 \mathrm{MeV} / \mathrm{m}$. The four curves correspond respectively to no pinned vortex, single pinned vortex (curve 1), double pinned vortex (curve 2) and triple pinned vortex (curve 3). The three arrows shows the position of the pinning point. . . . . . . . . . . . . . . . . . . . . .

4.5 In (a) simulations of the surface resistance as a function of the pinning point position are reported. For all three the curves $l=70$ $\mathrm{nm}$ and $U_{0}=1.1 \mathrm{MeV} / \mathrm{m}$. Curve $a$ considers only one variable pinning point position, curve $b$ one fixed and one variable pinning point positions and curve $c$ two fixed and one variable pinning point positions. In (b) the surface impedance as a function of the mean free path is reported in the condition of single (1), double (2) and triple (3) pinning per vortex flux line. . . . . . . . . . . . . . 
4.6 Frequency dependence of the surface resistance. in (a) the real part of the surface impedance is plotted as a function of the frequency, where curves 1,2 and 3 corresponds to one, two and three pinning points. In (b) the surface resistance calculated for a single pinning point at $q_{0}=2 \mathrm{~nm}$ is plotted as a function of the mean free path for increasing frequency values. . . . . . . . . . . . . . . . . . 74

4.7 Depinning frequency as a function of the mean free path. . . . . . 75

4.8 Extrapolated zero field data [10] and real part of the trapped flux surface impedance simulation. . . . . . . . . . . . . . . . . . . 77

5.1 Flow chart representing the self-consistent numerical code to calculate $h_{c 1}$. . . . . . . . . . . . . . . . . . . . . . . . . . . . .

5.2 Numerical solution of the Ginzburg-Landau equations in cylindrical symmetry for different values of $\kappa$. . . . . . . . . . . . . . . .

5.3 Flow chart representing the self-consistent numerical code to calculate $h_{s h}$. . . . . . . . . . . . . . . . . . . . . . . . . . . . .

5.4 Numerical solution of the mono-dimensional Ginzburg-Landau equations for different values of $\kappa$ and applied field $h=0.75$. . . . .

5.5 Numerical values of $h_{c 1}$ and $h_{s h}$ as a function of the GinzburgLandau parameter. . . . . . . . . . . . . . . . . . . . . . .

5.6 Experimental quench fields for different cavities as a function of $\kappa$ along with numerical values of lower critical and superheating fields. 100

5.7 T-map acquired just before the cavity quench. The red circle shows the quench location. . . . . . . . . . . . . . . . . . . . . .

5.8 T-map data acquired during the first power rise of cavity acc005_1. Highlighted with the red circle the quench spot. . . . . . . . .

5.9 Temperature variation at the quench location as a function of the accelerating field. . . . . . . . . . . . . . . . . . . .

6.1 The top graph shows $b_{v}$ of the image vortex outside the superconductor $\left(-\Phi_{0}\right)$ that interacts with the vortex $\Phi_{0}$ inside the superconductor. The bottom graph shows instead vortex $\Phi_{0}$ inside the superconductor that interacts with the magnetic induction profile.

6.2 Total force acting on the vortex as a function of the normalized position $x$. In (a) the force is calculated for increasing $\kappa$ and field $h=h_{c 1}(\kappa)$ while in (b) for constant $\kappa=2.5$ but increasing field. 
6.3 Gibbs free energy density as a function of the normalized position $x$. In (a) $g$ is calculated for increasing $\kappa$ and field $h=h_{c 1}(\kappa)$ while in (b) for constant $\kappa=2.5$ but increasing field. . . . . . . . . . .

6.4 LE- $\mu \mathrm{SR}$ data reported in [11]. The N-doped data is courtesy of Dr. A. Romanenko . . . . . . . . . . . . . . . . . . . . . .

6.5 Magnetic induction profiles at the surface - lower graph - and of the anti-vortex - upper graph - for a non constant $\kappa$ profile and vortex position at $x=0.6$. The green lines correspond to the $\kappa$ profile in the two cases. . . . . . . . . . . . . . . . . . . . . . . . . . .

6.6 Total force acting on the vortex as a function of the normalized position $x$. In (a) the force is calculated for increasing superficial $\kappa_{s}$ and constant field $h=h_{c 1}\left(\kappa_{b}\right)$ while in (b) for constant $\kappa_{s}=2.5$ but increasing field. The layer thickness is fixed at $d=15 \mathrm{~nm}$ and $k_{b}=1.04$. . . . . . . . . . . . . . . . . . . . . . . . . . . .

6.7 In (a) an example of force as a function of the position $x$ is plotted along with the $\kappa$ profile selected. In (b) and (c) the magnetic induction modulus of the image-vortex is plotted along with the $\kappa$ profile for constant $\kappa$ and dirty layer (non-constant $\kappa$ ) cases respectively. .

6.8 Gibbs free energy density as a function of the normalized position $x$. In (a) $g$ is calculated for increasing superficial $\kappa_{s}$ and constant field $h=h_{c 1}\left(\kappa_{b}\right)$ while in (b) for constant $\kappa_{s}=2.5$ but increasing field. The layer thickness is fixed at $d=15 \mathrm{~nm}$ and $k_{b}=1.04$. . .

6.9 Numerical calculations of force (a) and Gibbs free energy density (b) for different dirty layer thicknesses. Curves $a$ and $i$ correspond to the limiting cases of layer with infinitely small and infinitely large thickness respectively. The plotted functions are shifted one respect to the other by $5 \mathrm{~cm}^{-1}$ in (a) and by 2 in (b). The horizontal lines represent the zero for every particular function. . . . . . . . .

6.10 Comparison between the Gibbs free energy density calculated for a constant $\kappa=2.5$ profile and a for non-constant profiles with layers having $\kappa_{s}=2.5, \kappa_{b}=1.04$ and various thicknesses when $h=0.4>$ $h_{c 1}\left(\kappa_{s}\right)$. . . . . . . . . . . . . . . . . . . . . . . . . .

6.11 In (a) enhancement of the lower critical field as a function of $\kappa$ when the dirty layer is assumed. In (b) comparison between the Gibbs free energy density for constant $\kappa$ and dirty layer cases when the vortex starts to be stable in the material bulk $\left(g_{\infty}=0\right)$. . . . . . . .

6.12 SIMS profile for nitrogen of a N-doped cavity cut-out, and of a Ninfused witness sample. . . . . . . . . . . . . . . . . . . . . 
6.13 Quench data for N-doped and N-infused cavities as a function of $\kappa$ plotted along with the numerical calculations of the critical fields. The dotted lines correspond to the constant $\kappa$ values of $B_{c 1}$ and $B_{s h} .133$

6.14 Quality factor versus accelerating field measured for representative cavities treated with different recipes. . . . . . . . . . . . . .

7.1 Experimental setup for: (a) 1-cell cavities, (b) 9-cell fully dressed LCLS-II cavity. All the dimensions are given in millimeters [124]. .

7.2 $Q_{0}$ versus accelerating field curves acquired after a cool-down in compensated field before any quench. The red stars correspond to the $Q_{0}$ point acquired after quenching $>10$ times in compensated external magnetic field [124]. . . . . . . . . . . . . . . . . .

7.3 Quench study performed on cavity acc002: (a) variation of the residual resistance due to quenches in the presence of external magnetic field; (b) saturation of the residual resistance due to multiple quenches in the same external field. The labels " 0 " indicate the condition of compensated field, while the symbol "**" refers to multiple quenches. $\Delta R_{0}(H)$ points that correspond to the T-maps of Figure 7.4 are indicated with arrows and letters [124]. . . . . .

7.4 Evolution of the dissipation due to trapped field at the quench spot for acc002 after quenching: (a) a single, (b) two, (c) multiple times in 500 mOe, and after quenching (d) a single (e) two, (f) multiple times in compensated field. The symbol "*” identifies multiple quenches. All the T-maps were acquired at $E_{a c c}=18 \mathrm{MV} / \mathrm{m}[124]$. . . . .

7.5 Variation of the residual resistance of aes011 after single quenches for different values and orientations of the external magnetic field [124].

7.6 Perfect Meissner effect simulation for different orientation of the magnetic field. Figure (a) field applied along y, (b) along z and (c) along $\mathrm{x}$ direction $[124]$. . . . . . . . . . . . . . . . . . .

7.7 Residual resistance evolution of aes019 after quenching in different field values. Every point in the graph correspond to multiple quenches in the same applied field. The arrows indicates the data points that correspond to the T-maps of Figure 7.8. The labels "0" indicate the condition of compensated field [124]. . . . . . . . . 
7.8 T-map images acquired after the cavity aes019 was quenched in presence of external magnetic field with the following sequence of magnitudes: (a) $700 \mathrm{mOe}$, (b) zero field, (c) 1 Oe and (d) zero field. Such sequence shows the impossibility of $Q_{0}$ recovery after the cavity was quenched in 1 Oe. The most part of the trapped flux could not be annihilated quenching again in zero field. All the T-maps were acquired at $E_{a c c}=17 \mathrm{MV} / \mathrm{m}[124]$. . . . . . . . . . . . . . . .

7.9 Simulations of the magnetic field distribution around the quench spot: (a) before quench; (b) during quench. Color scale represents the ratio between the local magnetic field and the applied magnetic field. (c) T-map of aes019 after multiple quenches in 500 mOe (acquired at $\left.E_{a c c}=17 \mathrm{MV} / \mathrm{m}\right) ;(\mathrm{d})$ schematics of the thermometer positions [124]. . . . . . . . . . . . . . . . . . . . . . . . .

7.10 Sketch of the magnetic field trapped at the quench spot: (a) after quench in the presence of external magnetic field - the T-map shows a two-lobe shaped dissipation pattern; (b) after the external field cancellation; (c) trapped magnetic flux after the flux migration-the T-map shows two hot spots; (d) field compensated after the magnetic flux migration $[124]$. . . . . . . . . . . . . . . .

7.11 The numerical solutions of the vortex motion equation (Eq. 7.3) for the displacement $\Delta x$ as a function of time for different pinning potentials is plotted in (a). The magnetic field considered in the calculation was 1 Oe, with mean free path $100 \mathrm{~nm}$. The separatrix line between equilibrium and vortex migration is plotted in (b) (see Eq. 7.5). Different lines correspond to different mean free paths. The points refer to magnetic field $B$ and potential $U_{0}$ chosen for the numerical solutions in (a) [124]. . . . . . . . . . . . . . . . .

7.12 Redistribution of the magnetic flux. In figure (a) the difference between the T-map acquired after quenching in $700 \mathrm{mOe}$ and in $500 \mathrm{mOe}$ is reported, while in (b) between quenching in 1 Oe and in $700 \mathrm{mOe}$. The flux redistributes/adds from negative to positive values regions [124]. . . . . . . . . . . . . . . . . . . . 


\section{LIST OF SYMBOLS}

\begin{tabular}{|c|c|}
\hline Symbol & Definition \\
\hline$\hbar$ & Plank constant over $2 \pi$ \\
\hline$\kappa_{B}$ & Boltzmann constant \\
\hline$\varepsilon_{0}$ & electric permittivity of vacuum \\
\hline$\mu_{0}$ & magnetic permeability of vacuum \\
\hline$\omega$ & resonance angular frequency \\
\hline$Q_{0}$ & intrinsic quality factor \\
\hline$E_{a c c}$ & accelerating gradient \\
\hline$\lambda_{0}$ & penetration depth \\
\hline$\lambda$ & effective penetration depth \\
\hline$\xi_{0}$ & coherence length \\
\hline$\xi$ & effective coherence length \\
\hline$l$ & electron mean free path \\
\hline$\kappa$ & Ginzburg-Landau parameter \\
\hline$\Delta_{0}$ & superconducting gap parameter at $T=0$ \\
\hline$\Delta$ & superconducting gap parameter \\
\hline$H_{c}$ or $B_{c}$ & thermodynamic critical field or magnetic induction \\
\hline$H_{c 1}$ or $B_{c 1}$ & lower critical field or magnetic induction \\
\hline$H_{c 2}$ or $B_{c 2}$ & upper critical field or magnetic induction \\
\hline$H_{s h}$ or $B_{s h}$ & superheating field or magnetic induction \\
\hline$\alpha$ & superconductor-normal-conductor interface energy \\
\hline$f_{s}$ & free energy density of the superconductor \\
\hline
\end{tabular}




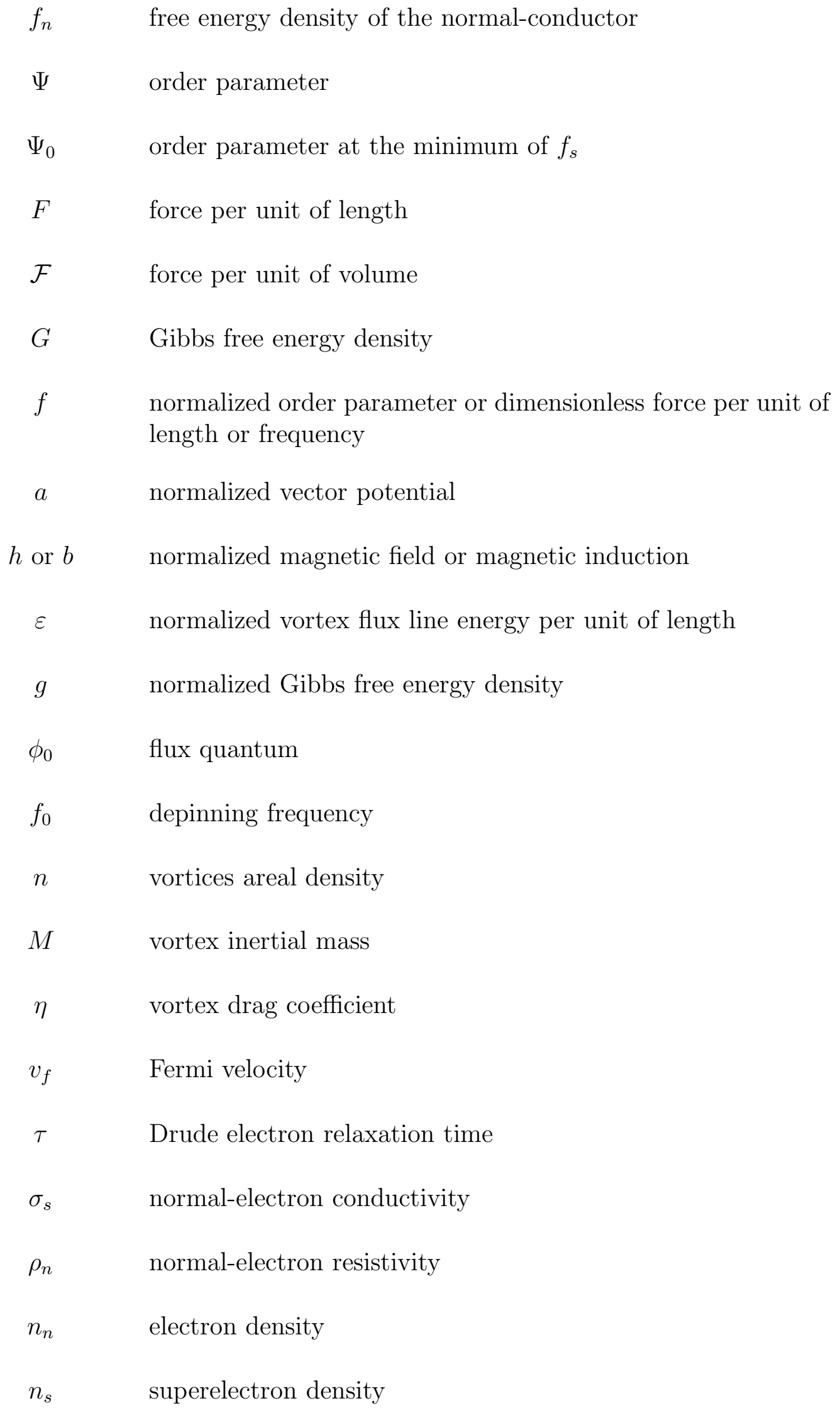




\begin{tabular}{|c|c|}
\hline$\nu_{n}$ & number of electrons \\
\hline$\nu_{s}$ & number of superelectrons \\
\hline$\nu$ & total number of current carriers \\
\hline$\beta$ & $\begin{array}{l}\text { ratio of velocity to the speed of light } c \text { or coupling parameter } \\
\text { for the input coupler }\end{array}$ \\
\hline$\beta_{t}$ & coupling parameter for the pick-up probe \\
\hline$\Gamma$ & reflection coefficient \\
\hline$P_{f}$ & forward power \\
\hline$P_{r}$ & reflected power \\
\hline$P_{t}$ & transmitted power \\
\hline$P_{d}$ & dissipated power by the cavity \\
\hline$P_{c}$ & dissipated power by the input coupler \\
\hline$P_{i} n$ & power leaking in the cavity through the input coupler \\
\hline$Q_{L}$ & loaded quality factor \\
\hline$Q_{c}$ & input coupler quality factor \\
\hline$Q_{t}$ & pick-up probe quality factor \\
\hline$\tau_{0}$ & cavity decay time \\
\hline$\tau_{L}$ & loaded decay time \\
\hline$\tau_{t}$ & pick-up probe decay time \\
\hline$R_{s}$ & surface resistance \\
\hline$R_{B C S}$ & Mattis-Bardeen surface resistance \\
\hline$R_{f l}$ & trapped flux surface resistance \\
\hline$R_{0}$ & intrinsic residual resistance \\
\hline
\end{tabular}




$\begin{array}{cl}E_{p} & \text { peak electric field } \\ B_{p} & \text { peak magnetic field } \\ R_{s h} & \text { shunt impedance } \\ R / Q & \text { R over } \mathrm{Q}\end{array}$




\begin{abstract}
Superconducting niobium accelerating cavities are devices operating in radiofrequency and able to accelerate charged particles up to energy of tera-electron-volts. Such accelerating structures are though limited in terms of quality factor and accelerating gradient, that translates - in some cases - in higher capital costs of construction and operation of superconducting rf accelerators. Looking forward for a new generation of more affordable accelerators, the physical description of limiting mechanisms in superconducting microwave resonators is discussed. In particular, the physics behind the dissipation introduced by vortices in the superconductor, the ultimate quench limitations and the quality factor degradation mechanism after a quench are described in detail.
\end{abstract}

One of the limiting factor of the quality factor is the dissipation introduced by trapped magnetic flux vortices. The radio-frequency complex response of trapped vortices in superconductors is derived by solving the motion equation for a magnetic flux line, assuming a bi-dimensional and mean free path-dependent Lorentzian-shaped pinning potential. The resulting surface resistance shows the bell-shaped trend as a function of the mean free path, in agreement with the experimental data observed. Such bell-shaped trend of the surface resistance is described in terms of the interplay of the two limiting regimes identified as pinning and flux flow regimes, for low and large mean free path values respectively. The model predicts that the dissipation regime - pinning- or flux-flow-dominated - can be tuned either by acting on the frequency or on the electron mean free path value. The effect of different configurations of pinning sites and strength on the vortex surface resistance are also discussed.

Accelerating cavities are also limited by the quench of the superconductive state, which limits the maximum accelerating gradient achievable. The accelerating field limiting factor is usually associated to the superheating field, which is intimately 
correlated to the penetration of magnetic flux vortices in the material. Experimental data for N-doped cavities suggest that uniform Ginzburg-Landau parameter cavities are statistically limited by the lower critical field, in terms of accelerating gradient. By introducing a Ginzburg-Landau parameter profile at the cavity rf surface - dirty layer - the accelerating gradient of superconducting resonators can be enhanced. The description of the physics behind the accelerating gradient enhancement as a consequence of the dirty layer is carried out by solving numerically the Ginzburg-Landau equations for the layered system. The enhancement is showed to be promoted by the higher energy barrier to vortex penetration, and by the enhanced lower critical field.

Another serious threat to the quality factor during the cavity operation is the extra dissipation introduced by the quench. Such quality factor degradation mechanism due to the quench, is generated by the trapping of external magnetic flux at quench spot. The purely extrinsic origin of such extra dissipation is proven by the impossibility of decrease the quality factor by quenching in a magnetic field-free environment. Also, a clear relation of the dissipation introduced by quenching to the orientation of the applied magnetic field is observed. The full recover of the quality factor by re-quenching in compensated field is possible when the trapped flux at the quench spot is modest. On the contrary, when the trapped magnetic flux is too large, the quality factor degradation may become irreversible by this technique, likely due to the outward flux migration beyond the normal zone opening during the quench. 


\section{CHAPTER 1 \\ INTRODUCTION}

\subsection{Why Superconducting Radio-Frequency Accelerators?}

High energy particle accelerators take advantage of radio-frequency (rf) acceleration technology in order to provide energy to charged particles. The main advantage of the rf technology is indeed to provide a progressive acceleration to charged particles, instead of a single-shot energy gain as typically achieved in electrostatic accelerators such as Cockcroft-Walton or Van der Graaff, minimizing the probability of voltage break-down.

The normal-conducting rf technology is conventionally copper dominated, and a large variety of resonating structures exits. Usually, copper cavities are water-cooled in order to extract the heat deposited on the cavity rf surface by the electromagnetic field, and keep the resistance at acceptable values. Cooling a normal-conducting resonator to low temperature, in order to minimize the cavity loss, is not always helpful because of the anomalous skin effect [12], that limits the minimum resistance of normal-conducting metals. Normal-conducting resonators are indeed not energetically efficient, a large part of the power fed to the structure is lost in Joule heating and not transferred to the beam.

A smart way to increase the energy efficiency of the resonator is to decrease the ohmic losses at the cavity surfaces taking advantage of the superconducting rf (SRF) technology. The SRF technology is bulk niobium dominated, since this latter is the elemental superconductor with the highest critical temperature. Also, metallic Nb can be shaped and welded with conventional methods to generate even complicated accelerating structures.

In applications demanding continuous wave (CW) operation or long puls- 
es/high duty cycles $(>1 \%)$, the SRF technology is required. Following that reported in $[13,14]$, let us consider the a simple accelerating structure $1 \mathrm{~m}$ long, operating in $\mathrm{CW}$ at $3 \mathrm{MV} / \mathrm{m}$ and $1.3 \mathrm{GHz}$ with $R / Q=1.8 \mathrm{k} \Omega$ (see Section 3.1.5). Assuming that the structure is made of copper with a quality factor of $Q_{0} \simeq 2 \times 10^{4}$ (see Section 3.1.4), the power dissipation is calculated to be $250 \mathrm{~kW}$. If we also consider the efficiency of the rf high power amplifier $\eta=0.6$, the total power consumption is then about $420 \mathrm{~kW}$.

Let us consider now an analogous structure $1 \mathrm{~m}$ long operating at $1.3 \mathrm{GHz}$ in $\mathrm{CW}$ at $3 \mathrm{MV} / \mathrm{m}$ but made in superconducting niobium operating at $2 \mathrm{~K}$. Since superconducting the $\mathrm{R}$ over $\mathrm{Q}$ ratio is usually chosen to be about three times lower than the normal conducting case [13], hence $R / Q=600 \Omega$. The power consumption due to Joule heating is way lower than the normal-conducting case, and equal to $0.89 \mathrm{~W}$. We should now consider also that the total power consumption depends on the cryogenic system needed to cool-down the cavity as well. Assuming a static power dissipation of $1 \mathrm{~W}$ per cavity (for TESLA type cavity cryomodules this value is of the order of $3 \mathrm{~W}$ per cryomodule [15]), Carnot efficiency $\eta_{c}=2 /(300-2)=0.0067$ and cryo-plant technical efficiency of about $\eta=0.2$, the total power consumption in the superconducting rf case is about $1.4 \mathrm{~kW}$. In $\mathrm{CW}$ operation the total power consumption is then about 297 times lower for the SRF technology with respect to the normal-conducting one.

It is important to point out that such gain in power consumption is in principle larger when lower frequencies are adopted. As it is discussed in Chapter 2, the resistance of superconductors is proportional to $\omega^{2}$. On the other hand, normalconductors have a surface resistance proportional to $\sqrt{\omega}$ in the normal skin effect regime [12]. Hence, the gain in lower values of dissipated power for SRF cavities decreases with increasing frequencies approximately as $\omega^{-3 / 2}$. In order to calculate the 
total power consumption, it is important to consider though that as the cavity frequency decreases, its dimension, and therefore the static power dissipation, increases with it.

Since their low dissipated power allows for large shunt impedance (see Subsection 3.1.5), superconducting rf cavities give the possibility of increasing the dimension of the beam pipes, assuring better beam stability. Larger beam pipes permits a more efficient higher order modes extraction, lower beam impedance and greatly reduces the probability of beam-loss and therefore of activation of the beam pipe.

\subsection{Performance Limitation Impact on the Cost of Accelerators}

Several factors must be considered in order to discuss the cost of high energy particle accelerators. In principle, three big cost driving contributions can be defined [16]: i) the civil engineering cost of the facility per unit of length, ii) the electric energy cost per unit of consumed power and iii) the accelerating technology cost per unit of beam energy.

The total project cost (TPC) — cost considering also components, conventional systems, R\&D, engineering design, management, contingency, over-head founds, site development, labor, etc.- can in principle be defined by the following phenomenological equation [16] that considers the sum of the three cost driving contributions defined above. Assuming SRF accelerating technology the TPC is:

$$
\mathrm{TPC} \approx 2 \mathrm{~B} \$ \sqrt{\frac{L}{10 \mathrm{~km}}}+10 \mathrm{~B} \$ \sqrt{\frac{E}{1 \mathrm{TeV}}}+2 \mathrm{~B} \$ \sqrt{\frac{P}{100 \mathrm{MW}}},
$$

where $L$ is the total length of the tunnel, $E$ the center-of-mass energy in colliders or energy per beam in linacs and $P$ the total ac power consumption of the facility. For example, the International Linear Collider (ILC), a superconducting rf $e^{+} e^{-}$linear collider, is estimated to have a cost around $7.8 \mathrm{~B} \$$ (in 2013), with a TPC range of $13-19 \mathrm{~B} \$[16]$. 


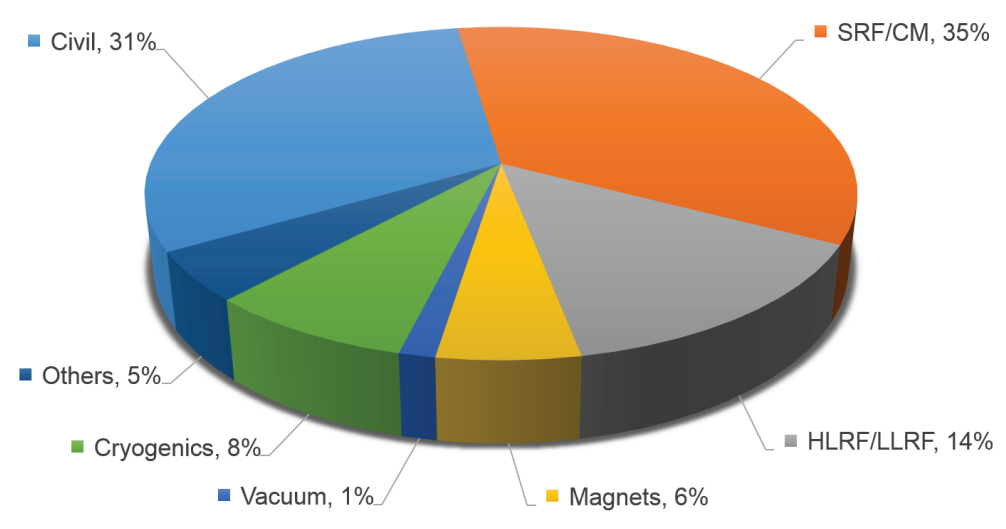

Figure 1.1. Pie diagram of the percentage contributions to the cost of the ILC. Data reported in [1]. Acronyms meaning: cryomodules (CM), high level rf (HLRF) and low level rf (LLRF).

The fundamental understanding of the SRF cavities limitations is extremely important in order to cut the cost of high energy accelerators like the ILC, and to limit the TPC as well. In Figure 1.1, the cost of the ILC divided into percentage subsections is reported.

Important to notice that the bigger cost slice (35\%) is associated to cavities and cryomodules production and assembly. The second big slice comes from the civil engineering side, for the construction of the tunnels and facilities. The third and fourth cost driving contributions are the HLRF/LLRF (klystrons, amplifiers, waveguides, power distribution, electronics, etc.) and the cryogenic cost respectively.

By understanding the fundamental limitations of SRF cavities, and by pushing their limit to higher accelerating gradients and lower power dissipation, a substantial reduction of the accelerator cost may be achievable. Higher operational gradients implies a shorter accelerator per same center-of-mass energy, ad therefore:

- shorter tunnel,

- less cryomodules and cavities, 
- less static heat load on the cryogenic plant,

- less klystrons (or solid state amplifiers), wave guide, distribution systems, etc.

The minimization of the ohmic dissipation of the cavity implies instead a substantial reduction of the dynamic heat load. The cavity can be operated at higher gradients and magnify the cost reduction contribution introduced by the higher gradients.

For example, increasing the nominal accelerating gradient of the ILC by $10 \mathrm{MV} / \mathrm{m}$ (from $31.5 \mathrm{MV} / \mathrm{m}$ to $41.5 \mathrm{MV} / \mathrm{m}$ ), form the civil engineering point of view the TPC is reduced of about $0.4 \mathrm{~B} \$$ (calculated with Eq. 1.1). The main limitation in achieving that is the current limitations of SRF cavities. Adopting conventional high gradient $\mathrm{SRF}$ technology, the dissipation at accelerating gradients larger than $31.5 \mathrm{MV} / \mathrm{m}$ is not sustainable in terms total power consumption (cryogenic cost included). Lower power dissipation is mandatory in order to affordably operate at high gradients.

Therefore, in order to allow for new powerful particle accelerators, fundamental $\mathrm{R} \& \mathrm{D}$ is needed to understand and overcome the current limitations in terms of power consumption and accelerating gradient, and push the SRF cavities performance to the next level.

\subsection{Thesis Organization}

The dissertation begins with a brief introduction on some aspects of superconductivity (Chapter 2). Most of the attention is addressed to the description of the main concepts needed to understand the following chapters, especially GinzburgLandau theory, magnetic vortex dynamics and Mattis-Bardeen surface impedance.

Chapter 3 contains the description of electromagnetic waves in resonators and basic definitions of quantities and concepts commonly used in the accelerating cavities 
community. The same chapter is also addressed to the description of measurement system and measurement process to test SRF cavities, as well as to an introduction on the SRF cavities performance for different surface finishing.

In Chapter 4 a first principles model of the vortex surface resistance is discussed with particular interest on the description of its dependence with the electron mean free path. The description is then also extended to define the resistance dependencies on frequency, pinning point position and pinning strength.

The fundamental accelerating gradient limitation of SRF cavities is discussed in Chapter 5, where the numerical calculation of the lower critical field and superheating fields, from the Ginzburg-Landau equations, are compared with experimental data for SRF cavities.

Always in the framework of the Ginzburg-Landau theory, the energetics of the vortex penetration is addressed in Chapter 6. In particular, the effect on the accelerating gradient enhancement due to a dirty layer at the rf surface is described and verified by the comparison with experimental data.

The study and the description of the extra dissipation associated to the quench phenomena is presented in Chapter 7 . The possibility of quality factor degradation recovery without thermally cycling the cavity is discussed.

Except for the introductory chapters, all the others are organized with an initial overview and a final summary of the results. The overall summary of the dissertation, that takes in consideration all the chapters, is presented in the conclusions section of Chapter 8. 
CHAPTER 2

\section{ELEMENTS OF SUPERCONDUCTIVITY}

\subsection{Historical Overview}

The phenomenon of superconductivity was discovered in the 1911 by the Duch physicist H. K. Onnes while measuring the resistivity of pure mercury at liquid helium temperatures $[17,18,19]$. He observed that at the temperature of $4.2 \mathrm{~K}$ the resistivity of mercury drops to zero, and that no power dissipation was associated to dc current flow. For such a reason this phenomenon was called superconductivity. Since then many new superconducting materials were discovered and several descriptions of the superconducting state where developed. Such remarkable discovery by Onnes had worth him the Nobel prize in Physics in the 1913.

The first model to explain the superconductivity phenomenon was proposed in the 1934 by J. C. Gorter and H. Casimir [20], where the superconducting state was described in analogy to the superfluidity of He-II below the lambda point $\left(T_{\lambda} \approx 2.17 \mathrm{~K}\right)$. In their description, when the temperature is decreased below the critical temperature $T_{c}$, a boson-like condensation takes place, and the electrons in the material may be represented as separated in two fluid-like groups: "superfluid" and "normalfluid" electrons.

As the temperature is decreased to zero, the number of "suprefluid" electrons $\left(\nu_{s}\right)$ increases, the number of "normalfluid" electrons $\left(\nu_{n}\right)$ decreases, while the total number of particles $(\nu)$ is conserved $\left(\nu_{s} / \nu+\nu_{n} / \nu=1\right)$. In order to explain the zero resistivity below $T_{c}$ in such scenario, we need to assume the "superfluid" electrons current as not affected by any resistance. Therefore, in the circuit analogy of a superconductor, "superfluid" electrons can be in principle thought as subjected only to a shunt inductor placed in parallel the "normalfluid" electrons resistor. When a 
dc current is flowing, it will be carried by "superfluid" electrons though the shunt inductance and the resistance will then be zero.

One year later, the two brothers F. and H. London described for the first time the electrodynamics of supercoductors by introducing the so-called London equations [21]. The great success of such equations resides in their good description of the Meissner-Ochsenfeld effect [22]. The perfect diamagnetic behavior of superconductors is explained by the introduction of the London penetration depth $\lambda_{0}$, as the characteristic exponential decay depth of the magnetic field in the superconductor:

$$
B(z)=B(0) e^{-z / \lambda_{0}} \quad ; \quad \lambda_{0}=\sqrt{\frac{m}{\mu_{0} n_{n} q^{2}}},
$$

where $m, n_{n}$ and $q$ are respectively the mass the number density and charge of the current carriers. This simple description of superconductivity is though unable to describe the superconductive transition.

In the 1950, the Soviet scientists V. L. Ginzburg and L. D. Landau proposed the first thermodynamic description of the superconducting transition [23]. The superconductive transition was framed as a second order transition, as introduced by L. D. Landau alone several years before [24], where the order parameter was identified as a macroscopic pseudo-wave function $\Psi$. One of the most remarkable findings of such description is the first definition of the coherence length $\xi(T)$, as the characteristic length of variation of the order parameter in the superconductor. Important to mention that the Ginzburg-Landau theory returns a temperature independent definition of the penetration depth of the field $\lambda_{0}$ in agreement with the London electrodynamics $[25]$.

Ginzburg and Landau introduced also the parameter $\kappa$, which is related to the purity of the material:

$$
\kappa=\frac{\lambda(T)}{\xi(T)}
$$


where $\lambda(T)$ is the temperature dependent penetration depth related to $\lambda_{0}$. Both $\lambda(T)$ and $\xi(T)$ temperature dependencies are valid in the limit $T \lesssim T_{c}$. In general $\kappa$ varies with the electron mean free path $l$ and defines the separation between type-I $(\kappa<1 / \sqrt{2})$ and type-II $(\kappa>1 / \sqrt{2})$ superconductors.

The formal description of the two different classes of superconductors (type-I and type-II) was proposed by A. A. Abrikosov seven years later [26]. He formalized the F. London's idea of magnetic flux quantization [27] in the framework of the GinzburgLandau theory, being able to describe the Mixed state of type-II superconductors. Such rigorous description of the type-II superconductivity was rewarded with the Nobel prize in Physics to A. A. Abrikosov in 2003 (shared with V. L. Ginzburg and A. J. Leggett "for pioneering contributions to the theory of superconductors and superfluids").

As a generalization of the London brothers electrodynamics, in the $1953 \mathrm{~A}$. B. Pippard proposed a new equation for the supercurrent, in which the current is related to an average of the vector potential over a region governed by the coherence length $\xi[28]$. Such new definition of the coherence length depends as well on the electron mean free path $l$, but was formulated independently from V. L. Ginzburg and L. D. Landau. The alternative definition of $\xi$ in the Pippard's electrodynamics is:

$$
\frac{1}{\xi}=\frac{1}{\xi_{0}}+\frac{1}{l}
$$

with $\xi_{0}$ the coherence length for the pure superconductor:

$$
\xi_{0}=a \frac{\hbar v_{F}}{\kappa_{B} T_{c}},
$$

where $a$ is a constant near to unity and $v_{F}$ the Fermi velocity.

Pippard did also introduce a new definition of the penetration depth $\lambda$, that is now assumed to be an effective penetration depth inversely proportional the the 
square root of the coherence length:

$$
\lambda=\lambda_{0} \sqrt{\frac{\xi_{0}}{\xi}}=\lambda_{0} \sqrt{1+\frac{\xi_{0}}{l}},
$$

where $\lambda_{0}$, the London penetration depth, is now representing the characteristic penetration depth of the clean superconductor.

The superconductor electrodynamics changes with the purity of the material, so that when the mean free path is large, $\xi_{0}<<l$ or $\lambda<<\xi$, then the Pippard nonlocal description is appropriate. In the opposite regime where $\xi_{0}>>l$ or $\lambda>>\xi$, the local London description shall instead be used. The Pippard's electrodynamics has a more general validity than the London electrodynamics, we may indeed consider the London theory as the limit of the Pippard theory for dirty superconductors $\left(\xi_{0}>>l\right)$.

The most complete and exhaustive description of conventional superconductors (low temperature superconductors) was published by J. Bardeen, L. N. Cooper and J. R. Schrieffer in the 1957 [29]. Such theory denominated BCS after its authors was worth them the Nobel price in physics in the 1972.

In the BCS theory, the interaction between electrons, which results from the virtual exchange of phonons, is attractive when the energy difference between the electrons energy states is smaller than the exchanged phonon energy. In such situation the Coulomb repulsive interaction is overcome and electrons that possess opposite spin and momentum may form the so-called Cooper pairs [30]. Even if the single electron follows the Fermi-Dirac statistics the Cooper pair is a Boson and therefore may condense following the Bose-Einstein statistics. The description of a Cooper pair is a two-body approximation of a many-body phenomenon where electrons interacts attractively with each other within a certain dimension, the coherence length $\xi_{0}$. Anyway, the coherence length may be thought as the dimension of a single pair, and 
it was identified as:

$$
\xi_{0}=\frac{\hbar v_{F}}{\pi \Delta_{0}} \approx \frac{\hbar v_{F}}{\kappa_{B} T_{c}}
$$

in agreement with the definition given by Pippard reported in Eq. 2.4. Here $\Delta_{0}$ is half of the superconducting energy gap at $T=0$, described in the following paragraphs.

The direct consequence of such attractive interaction between electrons is the gradual condensation of Cooper pairs at their ground state as the temperature is decreased below the critical temperature $T_{c}$. The fraction of electrons that cooperate in the formation of Cooper pairs are those with energy $\kappa_{B} T_{c} \approx \hbar \omega_{D}$ around the Fermi level $\left(E_{F}\right)$, where $\omega_{D}$ is the Debye frequency. Electrons that are inside such energy "crust" are pushed towards lower energies and gradually condense in Cooper pairs at the ground state with energy $E_{F}-\Delta_{0}$.

An energy gap, that separates condensed cooper pairs from depaired quasiparticles (not paired electrons), is then formed around the Fermi level, and the parameter $\Delta_{0} \approx \kappa_{B} T_{c}$ corresponds to half of the dimension of such a gap at $T=0$. The energy gap has quasi-constant dependence with the temperature, except near $T_{c}$ where it rapidly decreases to zero. A handy approximation is [31]:

$$
\Delta(T)=\Delta_{0} \sqrt{\cos \left(\frac{\pi}{2}\left(\frac{T}{T_{c}}\right)^{2}\right)} .
$$

The gap formation implies that in order to break Cooper pairs and promoting the formation of quasi-particles when $T<T_{c}$, the superconductor must absorb energy $\hbar \omega$ larger than $2 \Delta$. Also, the density of states $(N(E))$ is substantially modified and singularities are formed at the gap edges [25, 29].

Since Coopers pairs follow the Bose-Einstein distribution, a virtually infinite number of them can occupy the same energy level, hence the density of state of a 
superconductor has the form:

$$
N(E)=N(0) \frac{E}{\sqrt{E^{2}-\Delta^{2}}}
$$

where singularities arises when $|E|=\Delta$.

Important to point out that the BCS theory can describe exhaustively the Meissner state and the superconducting second order phase transition. Moreover, in the approximation $T \approx T_{c}, \mathrm{~L}$. P. Gor'kov demonstrated that the Ginzburg-Landau equations may be directly calculated from the BCS theory [32], meaning that the two different approaches are consistent.

\subsection{Ginzburg-Landau Theory}

As just introduced, the Ginzburg-Landau theory [23] is a phenomenological theory capable to describe the superconducting phase. Such theory was developed on the basis of the Landau concept of phase transition of the second order [24].

In such description of the superconducting phase transition, the order parameter was assumed equal to a complex quantity, namely the pseudo wave function $\Psi(\mathbf{r})$, where its absolute value squared is equal to the superelectrons density $|\Psi(\mathbf{r})|^{2}=n_{s}(\mathbf{r})$.

As for the common second order phase transition, the superconductive transition is described by a minimum in the free energy density as a function of the order parameter. In the approximation of little variations of the order parameter $\left(T \lesssim T_{c}\right)$ and in case of zero magnetic field throughout the superconductor, the free energy density can be expanded in powers of $|\Psi(\mathbf{r})|^{2}$, where $f_{n}$ is the free energy density of the normal conducting state in absence of magnetic field:

$$
f_{s}=f_{n}+\alpha(T)|\Psi(\mathbf{r})|^{2}+\frac{\beta(T)}{2}|\Psi(\mathbf{r})|^{4}+\ldots
$$

The expansion was done only on even powers of the order parameter since the system must be stable both at transition and in the superconductive state. This means that 


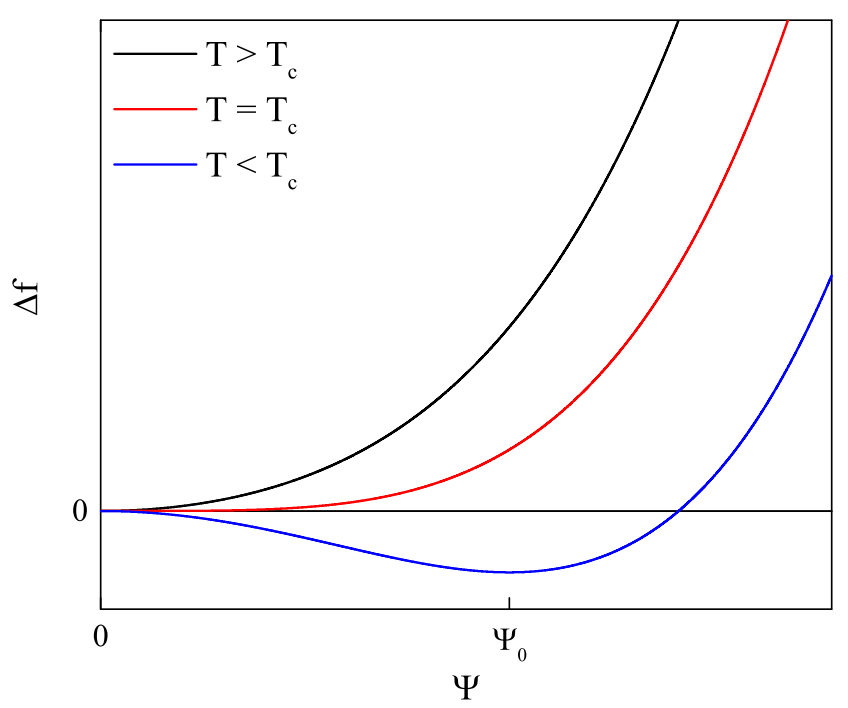

Figure 2.1. Variation of the free energy density as a function of the order parameter for normal-conducting phase $\left(T>T_{c}\right)$, superconductiong phase $\left(T<T_{c}\right)$ and at transition $\left(T=T_{c}\right)$.

for $T \geq T_{c}$ the free energy $f_{s}$ must have a minimum at $\Psi=0$.

In order to assure a minimum of $f_{s}$ at finite values of the order parameter in the superconductive state, we must set $\beta>0$. In such a way the variation of free energy density $\left(\Delta f=f_{s}-f_{n}\right)$ depends only on the sign of $\alpha$. In the normal-conducting phase $\left(T>T_{c}\right), \alpha>0$ and the minimum occurs at $\Psi=0$. On the opposite, in the superconducting phase $\left(T<T_{c}\right), \alpha<0$ and the minimum is found to be at:

$$
\left|\Psi_{0}\right|^{2}=-\frac{\alpha}{\beta}
$$

Evidently then, $\alpha(T)$ must change sign at $T_{c}$.

In Figure 2.1 the variation of free energy density is plotted against the order parameter. The value $\Psi_{0}$ corresponds to the minimum of $\Delta f$ in the superconducting state.

If we now allow for spatial variations of the order parameter (e.g. presence of a surface), and we assume also the presence of magnetic field in the superconductor, we must rewrite the free energy density expansion adding the correction to $f_{n}$ for 
finite magnetic field values $\left(B^{2} /\left(2 \mu_{0}\right)\right)$ and the kinetic energy density of the pairs $1 /\left(2 m^{*}\right)\left|\left(-i \hbar \nabla-e^{*} \mathbf{A}\right) \Psi\right|^{2}$ :

$$
f_{s}=f_{n}+\alpha(T)|\Psi(\mathbf{r})|^{2}+\frac{\beta(T)}{2}|\Psi(\mathbf{r})|^{4}+\frac{1}{2 m^{*}}\left|\left(\frac{\hbar}{i} \nabla-e^{*} \mathbf{A}\right) \Psi\right|^{2}+\frac{B^{2}}{2 \mu_{0}} .
$$

The parameters $m^{*}$ and $e^{*}$ are the mass and charge of a Cooper's pair, equal respectively to two times the mass and two times the charge of an electron, and $\mathbf{A}$ the vector potential describing the currents (and hence magnetic field) in the superconductor.

By implementing a variational procedure to minimize the free energy density $f_{s}$ defined in Eq. 2.11, the Ginzburg-Landau equations are derived. These two non-linear second order differential equations describe the distribution of the magnetic field and currents in the material, by returning the coupled quantities vector potential and order parameter that minimize the free energy density of the superconductor:

$$
\begin{aligned}
& \alpha \Psi+\beta|\Psi|^{2} \Psi+\frac{1}{2 m^{*}}\left(\frac{\hbar}{i} \nabla-e^{*} \mathbf{A}\right)^{2} \Psi=0 \\
& \mathbf{J}=\frac{e^{*} \hbar}{2 m^{*} i}\left(\Psi^{*} \nabla \Psi-\Psi \nabla \Psi^{*}\right)-\frac{e^{* 2}}{m^{*}} \Psi^{*} \Psi \mathbf{A},
\end{aligned}
$$

where $\mathbf{J}$ is the current density.

The Ginzburg-Landau equations (Eq. 2.12) just defined can be expressed in a more convenient dimensionless notation (see Appendix A):

$$
\begin{aligned}
& f-|f|^{2} f-\left(\mathbf{a}+\frac{i \nabla}{\kappa}\right)^{2} f=0 \\
& \nabla \times(\nabla \times \mathbf{a})+\frac{i}{2 \kappa}\left(f^{*} \nabla f-f \nabla f^{*}\right)+|f|^{2} \mathbf{a}=0,
\end{aligned}
$$

where $f$ and a are the dimensionless order parameter and vector potential respectively.

The dimensionless Ginzburg-Landau equations can be implemented in numerical calculations of the distribution of $f$ and and $\mathbf{a}$ for which the free energy density of the superconductor in minimized.

\subsection{Magnetic Flux Structures in Superconductors}


One of the most recognized phenomena that characterize the superconducting behavior, exception done for the zero dc resistivity, is the Meissner-Ochsenfeld effect [22]. Is such state the superconductor acts as a perfect diamagnetic material and expels the magnetic flux lines from its bulk, meaning that the magnetic induction in the superconductor is equal to zero.

Complete magnetic flux expulsion, as described by the Meissner-Ochsenfeld effect, is achievable when the magnetic field applied to the superconductor is relatively small, or when magnetic flux is not energetically favorable to be trapped in the the material. When magnetic field is applied, superconductors may show a rich variety of phenomena, most of them dependent on the type of superconductor (type-I or type-II).

2.3.1 Type-I Superconductors. Superconductors that are part of this category where the first discovered and are generally elemental metals such as mercury, lead, indium or aluminum.

In such class of superconductors the perfect diamagnetism (Meissner-Ochsenfeld effect) was observed to vanish once the magnetic field is increased above the so-called thermodynamic critical field $B_{c}$. This thermodynamic critical field is determined by equating the magnetic energy $B^{2} /\left(2 \mu_{0}\right)$ per unit of volume, associated to the magnetic pressure acting on the superconductor surface due to the expelled field, to the variation in free energy $\Delta f(T)=f_{n}(T)-f_{s}(T)$ related to the superconducting transition:

$$
\frac{B_{c}^{2}}{2 \mu_{0}}=\Delta f(T)
$$

Such thermodynamic critical field was observed to possess an empirical quadratic relation with temperature:

$$
B_{c}(T)=B_{c}(0)\left[1-\left(\frac{T}{T_{c}}\right)^{2}\right],
$$


where $B_{c}(0)$ corresponds to the thermodynamic critical field at $T=0$. In Figure 2.2a, the thermodynamic critical field dependence with the temperature is reported.

Below $B_{c}(T)$ the material is superconductive and in the Meissner state (all the magnetic flux is expelled from the material bulk). If the magnetic field or the temperature are such that $B>B_{c}(T)$, then the material is normal-conductive.

As described by the London electrodynamics [21], the Meissner effect may be explained by a simple exponential screening of the field due to supercurrents within a depth of the order of $\lambda_{0}$ from the superconductor's surface.

The Meissner effect can be simply described by means of the second London equation $[21]$ :

$$
\mathbf{J}_{\mathbf{s}}=-\frac{\mathbf{A}}{\mu_{0} \lambda_{0}^{2}},
$$

where $\mathbf{J}_{\mathbf{s}}$ is the superelectrons current density and $\mathbf{A}$ the vector potential. With the Maxwell equation $\nabla \times \mathbf{B}=\mu_{0} \mathbf{J}$, we can rewrite the second London equation as:

$$
\nabla^{2} \mathbf{B}=\frac{\mathbf{B}}{\lambda_{0}^{2}},
$$

with solution:

$$
B(x)=B(0) e^{-x / \lambda_{0}} .
$$

Which corresponds to an exponential decay of the field inside a length of the order of the penetration depth $\lambda_{0}$ (defined in Eq. 2.1). The magnetic field is therefore efficiently screened by the supercurrents inside $\lambda_{0}$ and the field excluded from the material bulk.

It was observed $[2,33]$, that when the aspect ratio of the superconducting sample is large in the direction perpendicular to the field direction, because of the demagnetization effect magnetic flux might penetrate in part of the sample even if the applied field is below $B_{c}(T)$. We can define a demagnetization coefficient $D$ such 

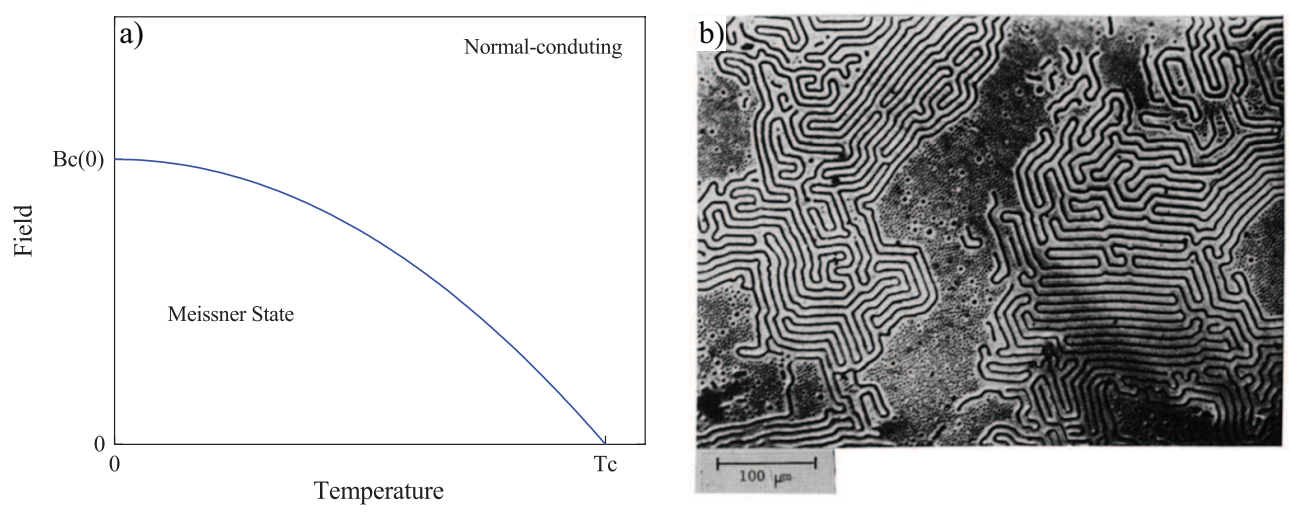

Figure 2.2. In (a) the thermodynamic critical field is plotted as a function of the temperature, while in (b) an example of intermediate state observed by bitter decoration in a single crystal lead foil [2].

that for the range

$$
B_{c}(T)(1-D)<B<B_{c}(T),
$$

the superconducting state must breakdown. In particular, normal-conducting arrangements of lamellae-like domains is formed in the regions where the enhancement of the magnetic field due to the demagnetization effect reaches values within the field range of Eq. 2.19. The magnetic field is therefore free to penetrate the normalconducting areas, but it is still expelled by the superconducting regions. Such condition is usually called intermediate state.

In Figure $2.2 \mathrm{~b}$ an example of intermediate state in a single-crystalline lead foil is reported [2]. The observation was done by bitter decoration technique. In black are shown the normal-conducting areas, while in white the superconducting ones.

In order to determine the domain pattern scale in the intermediate state, we should take into account the interface energy associated to the superconducting/normalconducting boundary. The superconducting behavior may be described by the order parameter $\Psi$, introduced in the previous section (Section 2.2). Such order parameter cannot vanish abruptly at the interface, on the contrary it approaches zero gradually within a certain distance from the superconducting phase surface. Such distance is 
of the order of the coherence length $\xi$, i.e. the characteristic variation length of the order parameter in the superconductor.

As a consequence we may expect a loss in condensation energy per unit of area in the superconducting phase due to the variation of $\Psi$ at the boundary, approximately equal to $\left(B_{c}^{2} / \mu_{0}\right) \xi$, that increases the superconducting/normal-conducting interface energy.

On the other hand, the magnetic field will penetrate in the superconducting region within a penetration depth $\lambda$ as described by the London and Pippard electrodynamics [21, 28]. We should then expect a reduction of condensation energy per unit area of the order of $\left(B_{c}^{2} / \mu_{0}\right) \lambda$. We can therefore define the interface energy per unit of area as:

$$
\alpha=\frac{B_{c}^{2}}{2 \mu_{0}}(\xi-\lambda)
$$

where $\delta=\xi-\lambda$ is positive for type-I superconductors since $\xi>\lambda$.

The superconductor then adjusts the domains interface area in order to minimize as much as possible $\alpha$. In particular, we can define two class of superconductors: those that have $\alpha>0$ are called type-I superconductors $(\kappa<1 / \sqrt{2})$, while those which possess $\alpha<0(\xi<\lambda)$ are called type-II superconductors $(\kappa>1 / \sqrt{2})$.

2.3.2 Type-II Superconductors. As just introduced, the second class of superconductors are called type-II superconductors. Material that are part of such category are generally metals alloys such like NbTi, MoRe or InSn, but also many simply compounds such as $\mathrm{Nb}_{3} \mathrm{Sn}, \mathrm{NbN}, \mathrm{V}_{3} \mathrm{Si}, \mathrm{MgB}_{2}$ or solid solutions like $\mathrm{NbN}_{\mathrm{x}} \mathrm{C}_{\mathrm{y}}$. In this class we found also some elemental metals like niobium or vanadium, ceramic high $T_{c}$ compounds such as $\mathrm{YBa}_{2} \mathrm{Cu}_{3} \mathrm{O}_{7-\mathrm{x}}, \mathrm{Bi}-2201, \mathrm{Bi}-2212$ or Bi-2223 and iron-based superconductors (pnictides) like LaOFeAs or SmFeAsO.

The main characteristic of type-II superconductors is their different behavior 

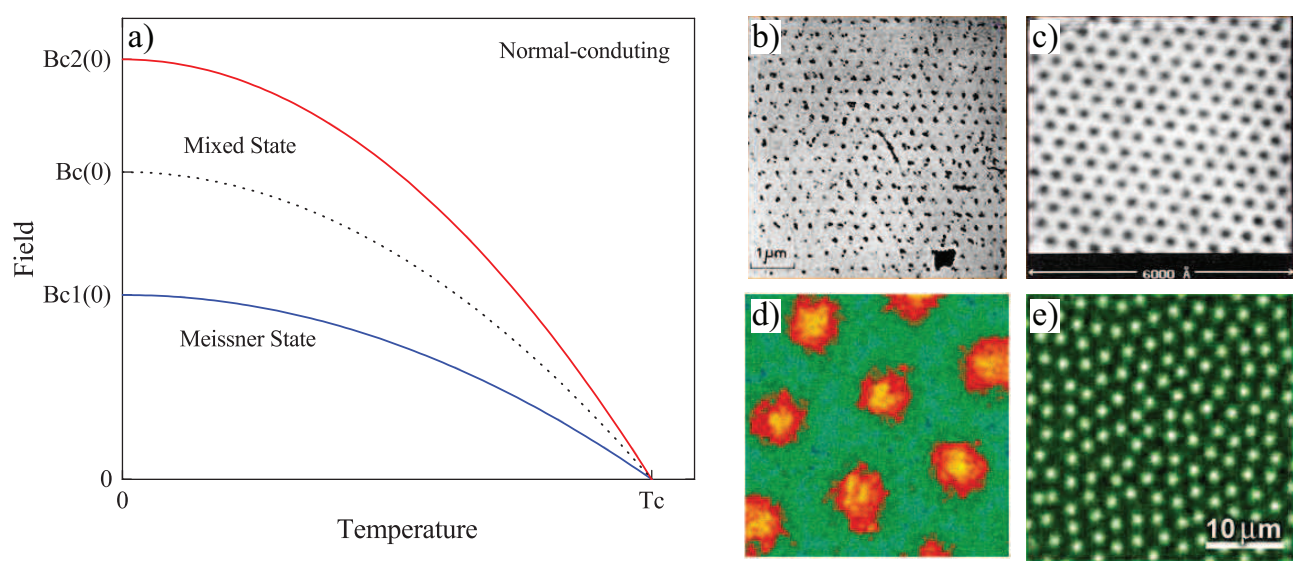

Figure 2.3. In (a) dependence of the lower and upper critical fields with temperature.

On the right, some examples of Mixed state are reported, where the vortex lattice was imaged by (b) bitter decoration of PbIn [3], (c) scanning tunnel microscopy of $\mathrm{NbSe}_{2}$ [4], (d) scanning tunnel microscopy of $\mathrm{MgB}_{2}$ [5] and (e) magneto-optical imaging of $\mathrm{NbSe}_{2}[6]$

in condition of applied magnetic field. Indeed, such class of materials usually present larger penetration depth than coherence length $(\xi<\lambda)$, that translates in a negative interface energy $\alpha$ (Eq. 2.20) between superconducting and normal-conducting phases. Direct consequence of $\alpha<0$ is the generation of a new superconducting phase (the so-called Mixed state), for which magnetic flux structures are actually stable in the superconductor independently on any demagnetization effect.

Type-II superconductors were discovered experimentally by L. V. Shubnikov et al. [34]. They observed that the superconductive state of alloys could survive up to larger magnetic fields that previously expected. Anyway, they had to wait until 1957 before their experimental observations could be explained, when A. A. Abrikosov [26] predicted the existence of the Mixed state by demonstrating that the Ginzburg-Landau equations (Eq. 2.12) allow solutions with periodical variations of the order parameter.

Abrikosov showed that in the Mixed state magnetic flux structures carrying a single flux quantum $\phi_{0}=h / 2 e=2.07 \cdot 10^{-15} \mathrm{~Wb}$ are stable and organized in a 
bi-dimensional hexagonal lattice. Such magnetic flux structures called vortices are stable in the superconductor when the applied magnetic field falls in between the following range:

$$
B_{c 1}(T)(1-D)<B<B_{c 2},
$$

where $B_{c 1}(T)$ and $B_{c 2}(T)$ are called respectively lower and upper critical fields. Below $B_{c 1}(T)(1-D)$, the superconductor is in the Meissner state and the magnetic flux is expelled from the bulk, while above $B_{c 2}(T)$ the material turns normal-conducting.

As the thermodynamic critical field, also $B_{c 1}(T)$ and $B_{c 2}(T)$ show a dependence on the temperature that in first approximation can be assumed quadratic as the empirical formula in Eq. 2.15. In Figure 2.3a, the temperature dependence of the lower and upper critical fields are reported. In Figure 2.3b, c, d and e some images of the Abrikosov vortex lattice in the Mixed state are reported.

2.3.3 Vortex Description. A magnetic flux vortex should be imagined as a cylindrical symmetric modulation of magnetic field, as shown in Figure 2.4a. Since the supercurrent $J_{s}$ is spinning around the vortex in order to screen the magnetic field, this latter decays radially with decay constant $\lambda$. Independently on $\lambda$, the magnetic flux carried by the vortex is quantized and equal to $\phi_{0}$ (as initially propose by $\mathrm{F}$. London [27]). In Figure 2.4a a schematic representation of a vortex is reported.

We should expect that such magnetic field profile is followed by a modulation of order parameter. In the non-local description of the vortex, a quantized flux line is represented as a modulation of the order parameter of the superconductor that tends to zero at the center of the vortex, and approaches to its finite value far from it [26].

Differently, in the local description [35], the vortex is described as a normal conducting core with radius $a$ of the order of the coherence length $\xi_{0}$, with superconducting currents spinning outside the core and screening the magnetic flux confined 
a)

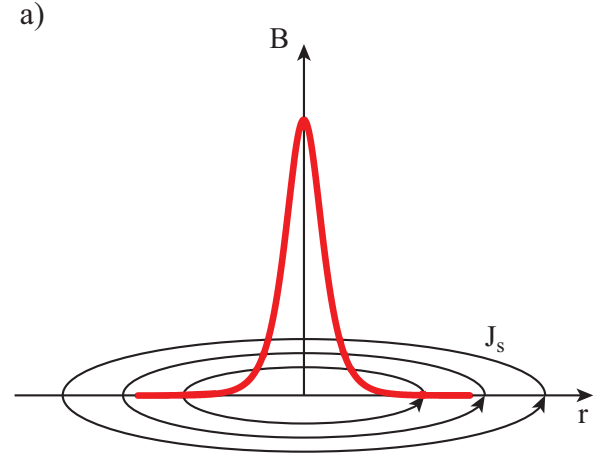

b)

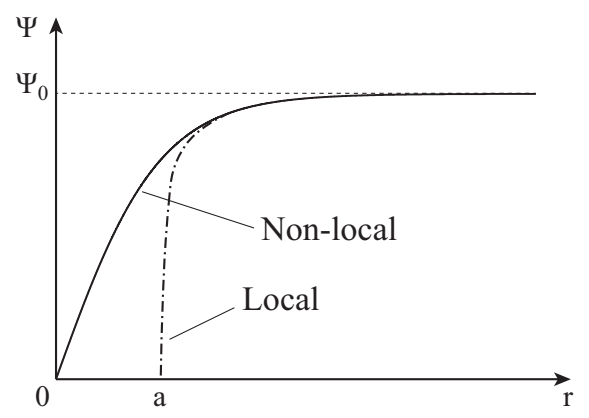

Figure 2.4. In (a) a schematic representation of a vortex, while in (b) a sketch of the order parameter variation as a function of the radius in the local and non-local model.

inside (a complete description of the local description is provided in [36]). A sketch of the two different descriptions of a vortex are reported in Figure 2.4b.

\subsection{Magnetic Flux Dynamics}

The magnetic flux dynamics in superconductors is extremely important in order to completely describe the dissipation in SRF cavities. Indeed, if during the superconducting transition some external magnetic field is applied to the cavity (or ambient remnant field is present), some magnetic flux might remain trapped even in the Meissner state because pinned at defects as described in [37]. Indeed, lots of experimental data $[10,38,39,40]$ indeed show that magnetic flux can be trapped in the cavity walls and increase the overall cavity dissipation.

Magnetic flux vortices trapped in the superconductor when subjected to currents or thermal gradients may indeed move and introduce extra dissipation. In this section a briefly explanation of the principal forces acting on a vortex are introduced.

2.4.1 Lorentz Force. Magnetic flux structures in the superconductor are subjected to the Lorentz force when currents (dc or rf) are flowing.

Let us assume a planar superconductor with surface parallel to the plane $x y$, 
and a localized flux $\phi$ (in case of a vortex $\phi=\phi_{0}$ ) crossing such superconductor with direction perpendicular to the surface along $\hat{z}$. If a current density $\mathbf{j}_{\mathbf{x}}$ is applied along the $x$ direction, the flux experiences a force per unit of length $\mathbf{F}_{\mathbf{L}}$ along the $\hat{y}$ direction:

$$
\mathbf{F}_{\mathbf{L}}=\mathbf{j}_{\mathbf{x}} \times \phi \hat{z}
$$

The Lorentz force is therefore always present when magnetic flux in the superconductor interacts with currents.

2.4.2 Thermal Force. When a thermal gradient is applied to the superconductor, another driving force than the Lorentz force exists: the thermal force.

Let us assume again a planar superconductor with surface parallel to the plane $x y$, and a localized flux quantum $\phi=\phi_{0}$ crossing such superconductor with direction perpendicular to the surface along $\hat{z}$. If a thermal gradient is applied to the superconductor along the $x$ direction, the vortex is expected to move in the opposite direction with respect the thermal gradient. The thermal force per unit of length $\mathbf{F}_{\mathbf{T}}$ is then $[33,41,42,43]$ :

$$
\mathbf{F}_{\mathbf{T}}=-S \nabla T
$$

where $S$ is the transport entropy per unit of length defined as [33, 41]:

$$
\begin{aligned}
S & =-\phi_{0} \frac{\partial H_{c, c 1}(T)}{\partial T} \\
& =2 \phi_{0} H_{0} \frac{T}{T_{c}}
\end{aligned}
$$

where $H_{0}$ is either the thermodynamic or the lower critical field at $T=0$ depending on the type of superconductor (I or II respectively).

The nature of this force is associated to the higher entropy density of the vortex core with respect the surroundings. The entropy density is also dependent on the temperature: higher temperature regions of the superconductor have higher 
entropy densities than the colder ones. This translates in a thermal force that acts by displacing vortices towards colder regions in order to increase the entropy of those areas [33].

2.4.3 Pinning Force. The pinning force is a restoring force acting on the magnetic flux lines in the superconductor.

Since the generation of areas containing magnetic flux translates into a loss of condensation energy, when the superconductor contains defects - areas where the condensation energy is smaller than the surrounding - or normal-conducting inclusions, then the magnetic flux prefers to sit in that positions than anywhere else, since the loss in condensation energy is smaller.

Thus, since these points are energetically favorable sites where the vortex may sit, they became pinning centers and the magnetic flux must pay some energy to move away from them. The pinning force is then associated to a pinning potential that is usually a rather complicated and hardly known function. The most simple analytic form for the pinning potential in approximation of little displacements is a parabola, and the pinning force may be described by a simple elastic restoring force.

In general though, flux lines are multiple-pinned in the superconductor, meaning that the approximate description of a simple pinning point potential might be misleading.

2.4.4 Viscous Drag Force. The viscous drag force, as the name says, acts against the motion of vortices and its proportional to the velocity of these latter:

$$
\mathbf{F}_{\mathbf{v}}=-\eta \dot{\mathbf{x}}
$$

where $\eta$ is the viscous drag coefficient.

The dissipation is observed both in dc and ac motion regimes. In particular, 
in dc regimes several experiment were performed in order to measure the value of $\eta$. One of the most accredited empirical formulas for $\eta$ was defined by Y. B. Kim. et al. [44]:

$$
\eta=\frac{3 \sigma_{n} \phi_{0}^{2}}{2 \pi^{2} \xi_{0} l},
$$

where $\sigma_{n}$ is the normal electrons conductivity and $l$ the electron mean free path.

In order to explain the mechanism underneath the viscous drag force, J. Bardeen and M. J. Stephen [36] describes the resistance due to flux motion by means of the vortex local description. In such framework, the viscous drag force is seen as generated by the Joule dissipation due to the normal-conductive currents induced in the vortex core by the flux motion.

The different approach adopted by M. Tinkham [45] is based on the relaxation time of the order parameter for a moving vortex. When a vortex moves in a superconductor at equilibrium, the order parameter must vary from its equilibrium value to zero (at the vortex center) in each instantaneous point at which the vortex is located. Moving from its previous to its next position, the vortex leaves a non-equilibriumorder-parameter trace behind it, i.e. a finite relaxation period passes before the order parameter returns to its equilibrium value. Because of the existence of such finite relaxation time, some condensation energy is lost, as if a force was acting against the flux motion - the viscous drag force.

Both the approaches gives comparable results of $\eta$ for $T \ll T_{c}$. While, in approximation $T \lesssim T_{c}$, L. P. Gor'kov and N. B. Kopnin [46] calculated $\eta$ connecting its changing rate to the variation in condensation energy, in the framework of the Ginzburg-Landau theory.

2.4.5 Magnus Force. The Magnus force was suggested by P. G. de Gennes [47, 48], and it is the analogous force acting on quantized vortices in liquid helium [33], but 
acting on magnetic vortices in superconductors.

If we assume a vortex moving with velocity $v$ in the $x$ direction and flux along $\hat{z}$, the Magnus force is directed along $\hat{y}$ perpendicular to the direction of the vortex motion.

$$
\mathbf{F}_{\mathbf{m}}=n_{s} e\left(\mathbf{v} \times \phi_{0} \hat{z}\right)
$$

where $n_{s}$ is the superelectrons density.

\subsection{Mattis-Bardeen Surface Impedance}

By meaning of the BCS theory, D. C. Mattis and J. Bardeen described the surface impedance of superconductors [49] $\left(Z_{s}\right)$ by assuming a complex conductivity $\sigma=\sigma_{1}-i \sigma_{2}$, as previously introduced by R. E. Glover and T. Tinkham [50]:

$$
\frac{Z_{s}}{Z_{n}}=\left(\frac{\sigma_{1}-i \sigma_{2}}{\sigma_{n}}\right)^{m}
$$

where $Z_{n}$ and $\sigma_{n}$ are the impedance and conductivity of the normal state, while $m$ is an exponent equal to $-1 / 2$ in the London limit, and to $-1 / 3$ in the Pippard limit [51].

The main result of the Mattis-Bardeen theory is the formalization of the imaginary and real part of the superconductor's conductivity:

$$
\begin{aligned}
& \frac{\sigma_{1}}{\sigma_{n}}=\frac{2}{\hbar \omega} \int_{\Delta}^{\infty}[f(E)-f(E+\hbar \omega)] g^{+}(E) d E \\
& \quad+\frac{1}{\hbar \omega} \int_{\Delta-\hbar \omega}^{-\Delta}[1-2 f(E+\hbar \omega)] g^{+}(E) d E, \\
& \frac{\sigma_{2}}{\sigma_{n}}=\frac{1}{\hbar \omega} \int_{\Delta-\hbar \omega ;-\Delta}^{\Delta}[1-2 f(E+\hbar \omega)] g^{-}(E) d E,
\end{aligned}
$$

where $f(E)$ and $f(E+\hbar \omega)$ are the Fermi-Dirac distributions that defines the quasiparticles state occupation at the energies $E$ and $E+\hbar \omega$ respectively, $\hbar \omega$ corresponds to the energy provided by the electromagnetic field, while the function $g^{ \pm}(E)$ is defined as:

$$
g^{ \pm}(E)=\frac{E^{2}+\Delta^{2}+\hbar \omega E}{\left[ \pm\left(E^{2}-\Delta^{2}\right)\right]^{1 / 2}\left[(E+\hbar \omega)^{2}-\Delta^{2}\right]^{1 / 2}}
$$




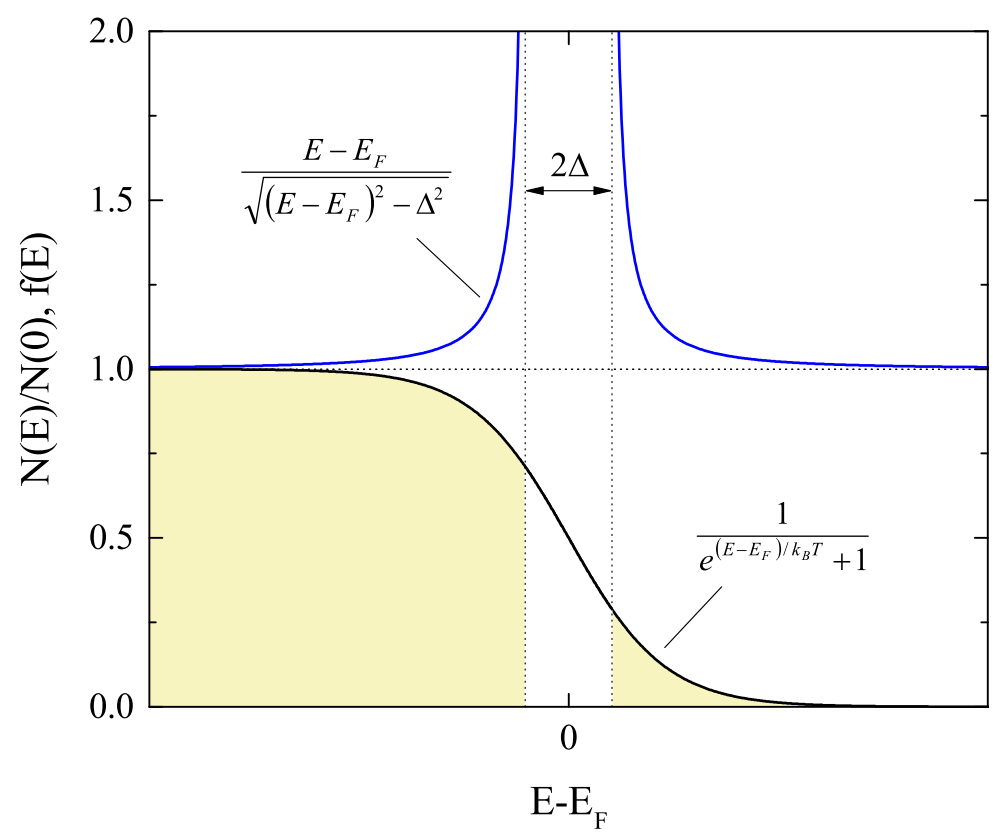

Figure 2.5. Density of states of a superconductor and Fermi-Dirac distribution as a function of the energy relative to the Fermi level.

The first integral of the real part of the conductivity in Eq. 2.29 defines the total number of thermally exited quasi-particle above the gap. The number of thermally exited quasi-particles $\nu_{n}$ scales with temperature approximately as:

$$
\nu_{n}(T) \sim \nu_{n} e^{-\frac{\Delta}{\kappa_{B} T}} .
$$

This means that when $T>0$ and $\Delta \approx \kappa_{B} T$, even if small, a certain number of quasiparticles not paired above the gap are present, as shown schematically by the yellow area in Figure 2.8. Such quasi-particle above the gap acts as normal-electrons and therefore introduce dissipation (and therefore a surface resistance) that varies with temperature $\left(R_{s} \sim e^{-\Delta / \kappa_{B} T}\right)$.

The second integral of the real part of the conductivity in Eq. 2.29 defines instead the dissipation introduced by pair breaking. When the absorbed energy $(\hbar \omega)$ is larger than $2 \Delta$ then pairs are broken and the population of quasi-particle increased. As for the thermal exited quasi-particles, broken pairs introduce extra dissipation and 


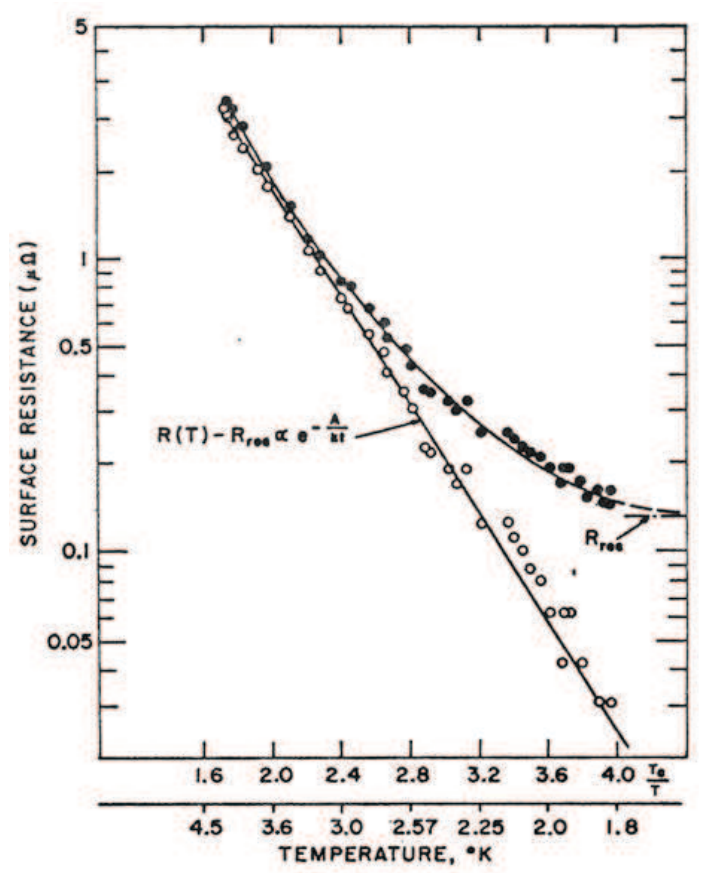

Figure 2.6. Surface resistance as a function of $T$ for $\mathrm{Pb}[7]$.

increase the surface resistance. Such contribution is though generally neglected, since for $\mathrm{SRF}$ cavities at $1.3 \mathrm{GHz} \hbar \omega<<2 \Delta\left(\Delta_{0} \approx 1.55 \mathrm{meV}\right.$ for niobium).

As defined by the Mattis and Bardeen theory, the surface impedance of a superconductor cannot be represented analytically with a simple formula, it has instead a rather complicated form. In particular, more parameters must be considered other than temperature $T$ and angular frequency $\omega$ to fully describe the surface resistance. Hidden inside the theory also the purity of the material plays an important role. Quasi-particles are indeed subjected to impurities scattering, and the Cooper pairs coherence length is affected by the material purity. The surface resistance has indeed a dependence on the mean free path and it is minimized for $l \approx \pi \xi_{0} / 2[52,53]$.

In order to overcome the complex notation of the Mattias-Bardeen surface resistance, an approximate formulation is usually adopted in order to fit the experimental data (in the SRF community people refer to the Mattis-Bardeen surface 
resistance as the BCS surface resistance, bacause of that I will from now on refer to it as $\left.R_{B C S}\right)$ :

$$
R_{B C S}(T, \omega, l)=\frac{A(l) \omega^{2}}{T} e^{-\frac{\Delta}{\kappa_{B} T}}
$$

where $A(l)$ is a free parameter that mainly depends on the mean free path. The surface resistance is therefore expected to decrease exponentially with the temperature (since also the thermally exited quasi-particle number decreases exponentially with $T$ ), but to increase with the square of the angular frequency $\omega$.

2.5.1 Residual Resistance. Experimental data shows that the surface resistance does decrease exponentially with $T$ as expected from the Mattis-Bardeen theory, but it was also observed that the superconductor presents a constant contribution to the surface resistance, independent on $T$, as swown in Figure 2.6.

When the temperature is small enough, the surface resistance assumes a constant value not theoretically explained. For such a reason the surface resistance is usually described by the following empirical formulation:

$$
R_{s}(T, \omega, l, B)=R_{B C S}(T, \omega, l)+R_{f}(l, B)+R_{0}
$$

where $R_{f}(l, B)$ is the surface resistance due to trapped magnetic flux in the cavity walls (described in Chapter 4 ), while $R_{0}$ is the intrinsic residual resistance due, but not only, to: niobium hydrides [54], niobium oxides [13] and damaged layer [55]. 


\section{CHAPTER 3}

\section{SUPERCONDUCTING ACCELERATING CAVITIES}

\subsection{Accelerating Cavities}

Superconducting accelerating cavities are resonant structures operating at radio-frequency (rf) or microwave frequencies and able to accelerate charged particles up to energies of the order of tera-electron-volts $(\mathrm{TeV})$.

Accelerating cavities are usually categorized as a function of their efficiency to transfer their stored electromagnetic energy to charged particles traveling through them. In general, three big classes of cavities exist: i) low $\beta$, ii) medium $\beta$ and iii) high $\beta$ cavities [13], where $\beta=v / c$ is the ratio between the velocity of the traveling particles $v$ and the speed of light.

Low $\beta$ cavities are usually implemented to accelerate heavy ions for nuclear physics experiments in machine such as FRIB, ATLAS and HIE-ISOLDE [56, 57, 58], or in the initial stage of proton machines such as PIP-II (ex PROJECT-X) [59]. Cavities that fall in this big class are the quarter-wave and half-wave resonators (QWR and HWR respectively). Such cavities are usually operating in transverse electromagnetic (TEM) modes at low frequencies (about 100-500 MHz).

Medium $\beta$ cavities, such as spoke resonators or elliptical cavities, are instead usually applied in the acceleration of protons and sometimes heavy ions for various applications such as "neutrino factories" (PIP-II [59]), spallation neutron sources (SNS and ESS [60]) and nuclear physics (FRIB [56]). The frequency range of such cavities usually ranges between about 500-800 MHz.

The last class of cavities is the so-called high $\beta$. Such cavities are design to reach high accelerating gradients, and are mostly used in order to accelerate particles to relativistic speeds in high energy physics machines like LHC or ILC $[61,62]$. High 


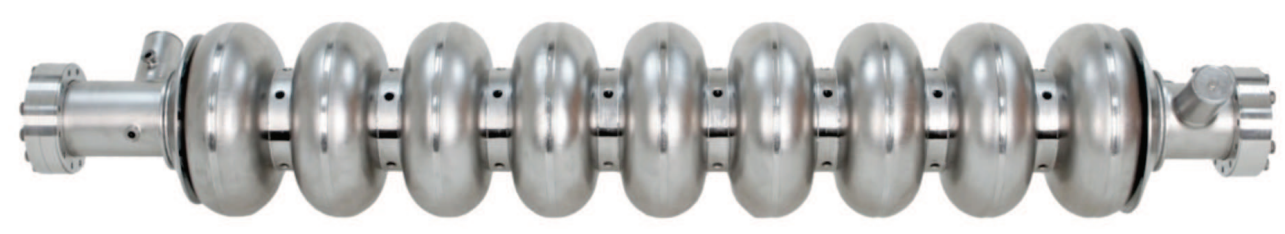

Figure 3.1. A 1.3 GHz TESLA-type nine-cells cavity [8].

$\beta$ cavities are also implemented in free electron lasers (FELs), such as EXFEL or LCLS-II $[63,64,65]$. High $\beta$ cavities operates in the range of few $\mathrm{GHz}$ and have elliptical or of elliptical-derived shape. The best examples are TESLA cavities [15], shown in Figure 3.1, or Ichiro cavities [66].

3.1.1 Electro-Magnetic Waves in the Cavity. The full analytic description of the electromagnetic field exited inside a resonator is usually complicated since the geometry can be very complex. In this subsection the electromagnetic field distribution inside a simple resonator without beam pipes is described. For this purpose the geometry chosen is the "pillbox" cavity (single- and multi-cells "pillbox" cavities are showed in Figure 3.2a) - a hollow cylinder with perfect conducting walls and filled with vacuum.

The electromagnetic field distribution in the resonator is described by the wave equation which is direct consequence of the Maxwell equations:

$$
\left(\nabla^{2}-\frac{1}{c^{2}} \frac{\partial^{2}}{\partial t^{2}}\right)\left\{\begin{array}{c}
\mathbf{E} \\
\mathbf{H}
\end{array}\right\}=0,
$$

where $c^{2}=1 /\left(\varepsilon_{0} \mu_{0}\right)$ is the speed of light squared. In this description, the cylindrical system of coordinates is adopted since more indicated to solve the problem than a cartesian one. Therefore, the electric and magnetic fields along the $z$ axis are defined as: $E_{z}=E_{z}(\rho, \varphi, z, t)$ and $H_{z}=H_{z}(\rho, \varphi, z, t)$. The coordinates $\rho, \varphi$ and $z$ represent respectively the radius, the polar angle and the length, as shown in Figure $3.2 \mathrm{~b}$. 
The general solution for electric and magnetic fields along the $z$ direction inside the resonator is found solving the wave equation in Eq. 3.1 by meaning of the separation of variables (the detailed calculation is reported in [67]):

$$
\left\{\begin{array}{c}
E_{z}(\rho, \varphi, z, t) \\
H_{z}(\rho, \varphi, z, t)
\end{array}\right\}=\left\{\begin{array}{c}
E_{0} \\
H_{0}
\end{array}\right\} J_{n}(\beta \rho)[A \sin (\kappa z)+B \cos (\kappa z)] e^{ \pm i n \varphi} e^{ \pm i \omega t}
$$

where $\beta^{2}=(\omega / c)^{2}-\kappa^{2}$ are the eigenvalues of the radial equation, with $-\omega^{2}$ and $-\kappa^{2}$ the separation constants of temporal and longitudinal problems respectively, $-n^{2}$ the separation constant of the angular problem and $J_{n}(x)$ is the $n$-th Bessel function, with $n=0,1,2 \ldots$. The two constants $A$ and $B$ are determined by applying the boundary conditions, while $E_{0}$ and $H_{0}$ are the maximum values of magnetic field and electric field respectively. The $n$-th Neumann function (the second solution of the eigenvalues problem) was instead neglected since diverging for $x \rightarrow 0$.

Considering the interface between the perfect conductor and the vacuum, the tangential component of the electric field $\mathbf{E}$, and the normal component of the magnetic field $\mathbf{H}$ must be continuous across the boundary. The following boundary conditions at the surface are then required:

$$
\hat{n} \times \mathbf{E}=0, \quad \hat{n} \cdot \mathbf{H}=0 .
$$

Such boundary conditions are respected only by two classes of field configuration inside the cylindrical resonator: $T M$ (transverse magnetic $\left.-H_{z}=0\right)$ and $T E$ (transverse electric $\left.-E_{z}=0\right)$ modes. The TM and TE solutions are found respectively by picking $E_{z}$ or $H_{z}$ in Eq. 3.2.

Accelerating cavities are resonators that exploit modes with axial electric field in order of accelerate charged particles, therefore only the modes TM will be considered. The particular solution of $E_{z}(\rho, \varphi, z, t)$ is calculated by applying the TM boundary conditions in Eq. 3.3 to the general solution of Eq. 3.2. Once $E_{z}$ is known, 


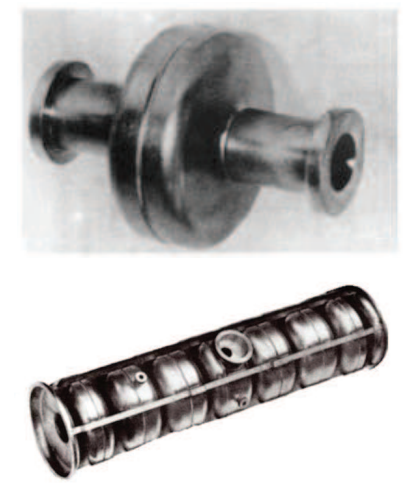

a)

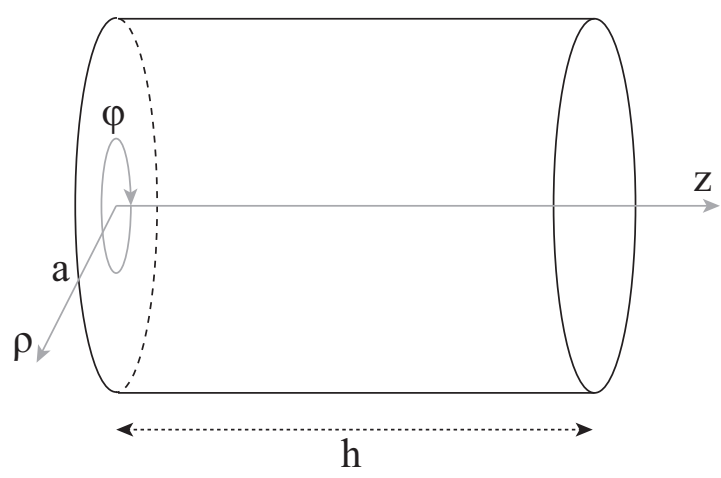

b)

Figure 3.2. In (a) a single- and a multi-cells HEPL "pillbox" cavity [9] (the pictures are courtesy of Prof. H. Padamsee) . In (b) a schematic representation of a "pillbox" cavity of radius $a$ and length $h$ without beam pipes and of the system of coordinates used in the calculation.

the transverse components of the electric field and the magnetic field in the resonator for the all the TM modes can be calculated by meaning of the following equations (derived in [67]):

$$
\begin{aligned}
& \mathbf{E}_{\mathbf{t}}= \pm \frac{i \kappa}{\beta^{2}} \nabla_{t} E_{z} \\
& \mathbf{H}_{\mathbf{t}}=\frac{i \omega \mu}{\beta^{2}} \hat{z} \times \nabla_{t} E_{z} .
\end{aligned}
$$

The full set of $\mathrm{TM}_{n l m}$ modes in the pillbox cavity can now be calculated, where the integers $n \geq 0, l \geq 1$ and $m \geq 0$ measure the number of times the field changes sign along the $\varphi, \rho$, and $z$ directions respectively. Furthermore, the resonant frequency for each $\mathrm{TM}_{n l m}$ mode is [67]:

$$
\omega_{n l m}=c \sqrt{\left(\frac{\alpha_{n l}}{a}\right)^{2}+\kappa^{2}} ; \kappa=\frac{m \pi}{h},
$$

where $\alpha_{n l}$ is the $l$-th node of the $n$-th Bessel function, while $a$ and $h$ the radius and length of the resonator as shown in Figure 3.2b.

The fundamental TM mode (with the lower frequency) is described by $n=0$, $l=1$ and $m=0\left(\mathrm{TM}_{010}\right)$, and it is the most suitable in order to accelerate charged 
a)

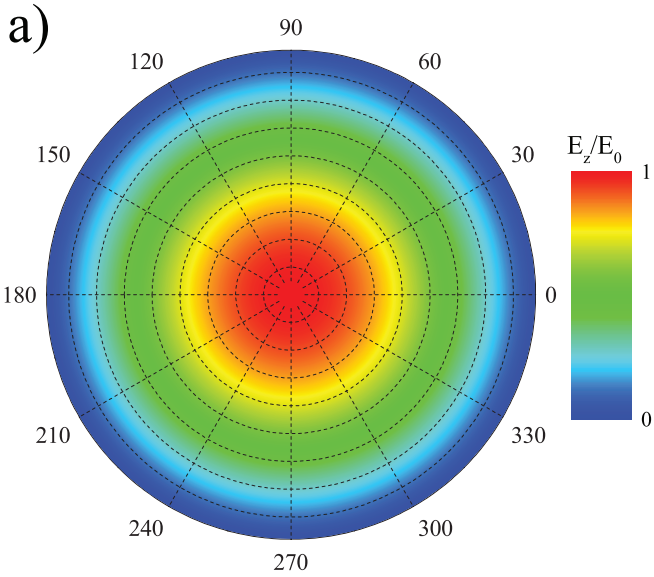

c)

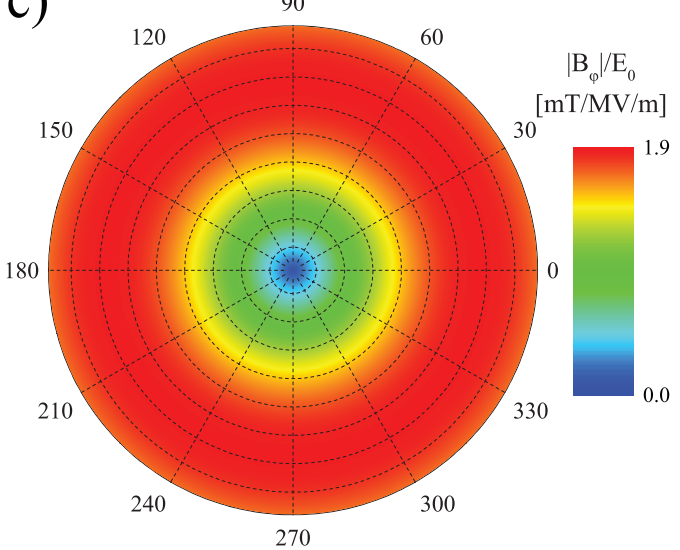

b)

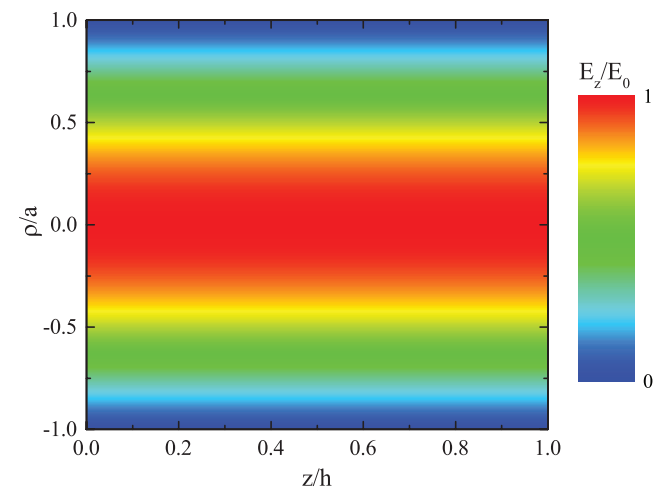

d)

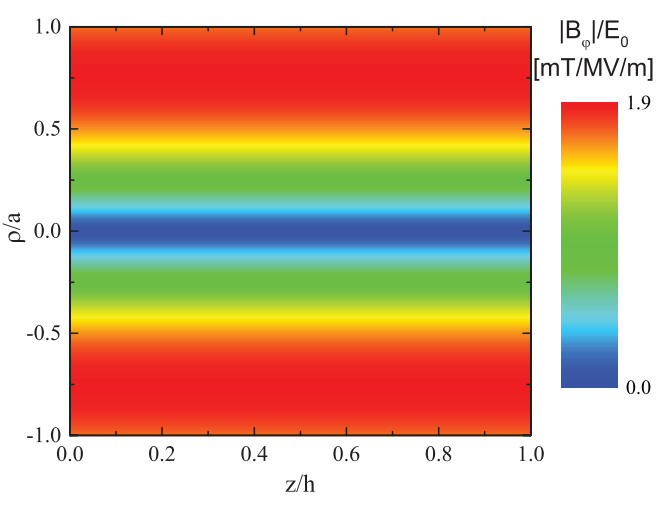

e)

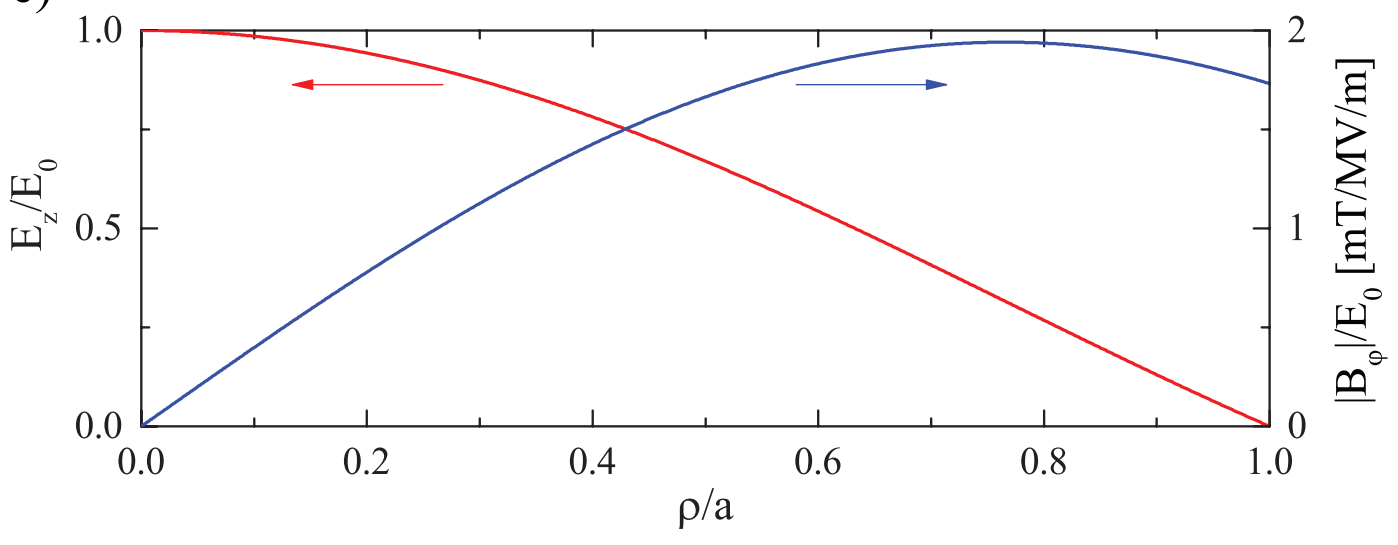

Figure 3.3. Polar and axial plots of the axial electric field and transverse magnetic field for the mode $\mathrm{TM}_{010}$. Red correspond the high values of $E$ or $B$, while blue to zero. 
particles. Its resonance frequency is given by Eq. 3.5:

$$
\omega_{010}=\frac{2.405 c}{a}
$$

and the correspondent electric and magnetic fields, calculated by means of Eq. 3.2, 3.3 and 3.4 , are:

$$
\begin{aligned}
& E_{z}=E_{0} J_{0}\left(\frac{2.405}{a} \rho\right) e^{ \pm i \omega_{010} t} \\
& H_{\varphi}=-i \varepsilon_{0} c E_{0} J_{1}\left(\frac{2.405}{a} \rho\right) e^{ \pm i \omega_{010} t}
\end{aligned}
$$

In Figure 3.3, the polar and axial plots of the mode $\mathrm{TM}_{010}$ for the axial electric and transverse magnetic fields are reported. Figure 3.3a and b show the electric field that as expected is maximized at the center of the resonator where the beam travels. In order to respect the boundary conditions in Eq. 3.3, the electric field is zero at the cylindrical walls, meaning that the first zero of the zeroth-order Bessel function coincides spatially with the cylinder radius. In Figure 3.3e, the radial profile of the field is reported for clarity.

The transverse magnetic field is instead zero at the cavity center and it increases radially towards the cavity walls as shown in Figure 3.3c, $\mathrm{d}$ and e. The magnetic field of the $\mathrm{TM}_{010}$ mode is indeed radially described by the first-order Bessel function which is zero at the origin. The maximum value of the transverse magnetic field falls at $\rho / a \approx 0.8$.

3.1.2 Accelerating Gradient. The accelerating voltage of a superconducting cavity can be defined as the ratio between the maximum energy gained by a charged particle through the cavity gap $d$ and the particle charge. The total energy gained by the particle traveling throughout the resonator is then described by:

$$
\Delta W=q \int_{-d / 2}^{d / 2} E(0, z, t) d z,
$$


where the electric field experienced by a particle with velocity $v$ on the axis of the cavity is a time-dependent function,

$$
E(0, z, t)=E(0, z) \cos [\omega t(z)+\varphi] .
$$

Here the time $t(z)=\int_{0}^{z} 1 / v(z) d z$ corresponds to the particle travel time, nominally the time the particle needs to move from 0 to the position $z$, and $\varphi$ identifies the phase of the particle arriving at the cavity center with respect to the oscillating field.

We can now express the energy gain as:

$$
\Delta W=q V_{0} T \cos \varphi,
$$

where the the axial accelerating voltage $V_{0}$ is defined as the integral of the axial electric field over the gap length:

$$
V_{0}=\int_{-d / 2}^{d / 2} E(0, z) d z,
$$

while $T$ corresponds to the transit-time factor [68], i.e. a measure of the reduction in the energy gain caused by the sinusoidal time variation of the field in the gap (regardless of the phase $\varphi$ ). For a even mode in the cavity with respect the cavity center (e.g. $\mathrm{TM}_{n l 0}$ ), the transit-time factor has form:

$$
T=\frac{\int_{-d / 2}^{d / 2} E(0, z) \cos (\omega t(z)) d z}{\int_{-d / 2}^{d / 2} E(0, z) d z} .
$$

Usually the particles velocity can be approximated constant throughout the gap and $v(z) \approx v$. Therefore $\omega t(z) \approx \omega z / v=\omega z / \beta c$, with $\beta=v / c$. The transit-time factor is then:

$$
T=\frac{\int_{-d / 2}^{d / 2} E(0, z) \cos \left(\frac{\omega z}{\beta c}\right) d z}{\int_{-d / 2}^{d / 2} E(0, z) d z}
$$

Since accelerating cavities are tested in absence of a particles beam, it is useful to define the maximum average accelerating gradient $E_{a c c}$ simply as:

$$
E_{a c c}=\frac{\Delta W}{q d}=\frac{V_{0} T}{d},
$$




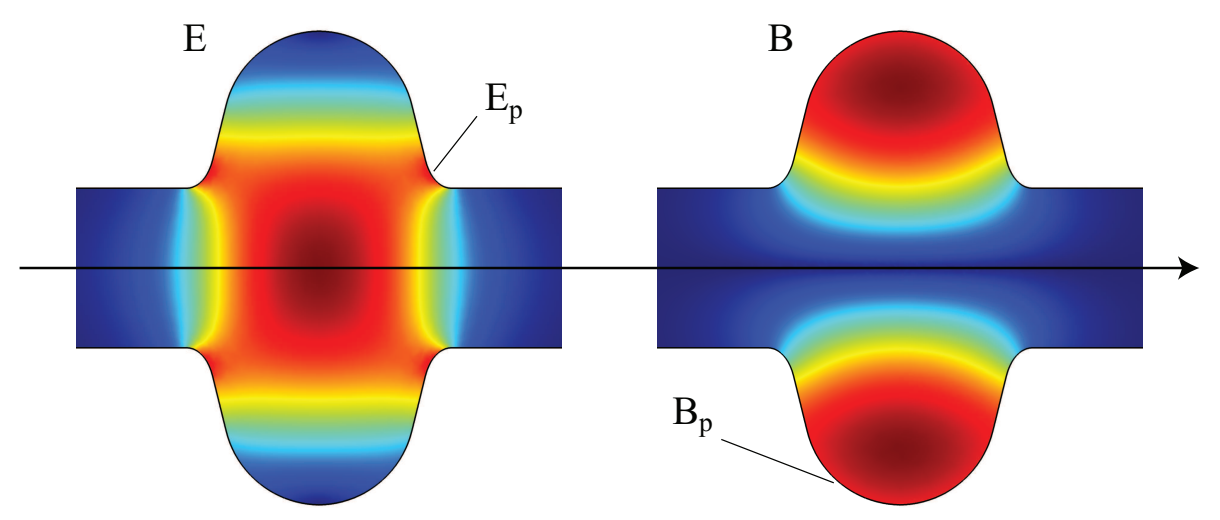

Figure 3.4. Electric and magnetic field simulations for a TESLA type elliptical cavity.

assuming that $\varphi=0$, as if the particle would arrive at the cavity center when the field is at a crest. When multi-cells cavities are considered, the gap dimension $d$ corresponds to the total active length of the cavity.

3.1.3 Peak Fields. Since in this dissertation we are dealing with superconducting accelerating cavities, the accelerating gradient is not the correct parameter needed to study the superconducting properties of the resonator. In general, accelerating cavities may be pushed in gradient up to a certain limit, at which the superficial magnetic field is not bearable anymore by the superconductor. For this reason, we should introduce two important parameters that defines the field level at the cavity surface: the peak electric and magnetic fields ( $E_{p}$ and $B_{p}$ respectively). In particular, $E_{p}$ is found at the cavity irises, while $B_{p}$ in the equatorial region, as shown in Figure 3.4.

Subject of this dissertation are elliptical cavities with TESLA geometry [15], which possess the following $E_{p} / E_{a c c}$ and $B_{p} / E_{a c c}$ ratios:

$$
\begin{aligned}
\frac{E_{p}}{E_{a c c}} & =2.0 \\
\frac{B_{p}}{E_{a c c}} & =4.26 \mathrm{mT} /(\mathrm{MV} / \mathrm{m}) .
\end{aligned}
$$

These two ratios are very important in order to design new accelerating struc- 
tures. Since they depends only on the cavity geometry, they may be used as figures of merit in order to characterize a particular accelerating structure.

3.1.4 Quality Factor. In order to sustain the electromagnetic field, the screening currents flow in the first tents of nanometers of material. As discussed in Chapter 2, even if small, superconductors always possess a finite value of surface resistance, and the screening currents will dissipate power.

Assuming an average surface resistance $R_{s}$ allover the cavity surface $\Sigma$, we can calculate the power dissipated by Joule heating as:

$$
P_{d}=\frac{1}{2} R_{s} \int_{\Sigma}\left|\mathbf{H}^{2}\right| d \Sigma
$$

where $\mathbf{H}$ is the local magnetic field at the cavity surface.

If the cavity is fed with a rf signal not all the power is dissipated by Joule heating, a big part of it is stored as electromagnetic energy in the resonator volume $V$. Such stored energy is defined as:

$$
U=\frac{1}{2} \mu_{0} \int_{V}\left|\mathbf{H}^{2}\right| d V
$$

When the cavity is not driven by an external rf signal, the dissipated power $P_{d}$ corresponds to the stored energy variation in the resonator $\left(P_{d}=-d U / d t\right)$. The energy in the resonator will then decay with a characteristic decay time $\tau_{0}$ :

$$
U(t)=U_{0} e^{-t / \tau_{0}}
$$

where the decay time can be defined in terms of quality factor (Q-factor) $Q_{0}: \tau_{0}=$ $Q_{0} / \omega$. We can therefore define the quality factor as the ratio of the energy gain per rf period and dissipated power:

$$
\begin{aligned}
Q_{0} & =\frac{\omega U}{P_{d}}=\frac{\omega \mu_{0} \int_{\tau}\left|\mathbf{H}^{2}\right| d \tau}{R_{s} \int_{\Sigma}\left|\mathbf{H}^{2}\right| d \Sigma} \\
& =\frac{g}{R_{s}}
\end{aligned}
$$


where $g$ is the geometrical factor defined as:

$$
g=\frac{\omega \mu_{0} \int_{\tau}\left|\mathbf{H}^{2}\right| d \tau}{\int_{\Sigma}\left|\mathbf{H}^{2}\right| d \Sigma}
$$

which depends only on the cavity geometry and for TESLA type cavities has value $270 \Omega[15]$.

Important to keep in mind that the Q-factor defines also the ratio between the resonance frequency and the bandwidth of the resonance peak: $Q_{0}=\omega / \Delta \omega$.

3.1.5 Shunt Impedance. The shunt impedance defines the effectiveness to produce an axial voltage $V_{0}$ in the cavity volume per amount of dissipated power. Such quantity is therefore of central importance in order to define the field level in the cavity and the energy transfer efficiency from the cavity to the beam.

In general, there are many definitions of shunt impedance $[13,68]$, in this dissertation the standard accelerator community definition is adopted:

$$
R_{s h}=\frac{V_{0}^{2}}{P_{d}}
$$

where $P_{d}$ is the dissipated power by the cavity.

Such definition of shunt impedance does not consider the variation of the field as seen by the particle beam passing through the cavity, it is therefore necessary to define the effective shunt impedance $R$ as:

$$
R=\frac{\left(V_{0} T\right)^{2}}{P_{d}}=\frac{\left(E_{a c c}\right)^{2}}{P_{d} / d^{2}} \quad, \quad E_{a c c}=V_{0} T
$$

where $T$ is the transit time factor as defined in Eq. 3.13 and $d$ the cavity active length (for nine-cells TESLA type cavities $d=1.038 \mathrm{~m} \mathrm{[15]).}$

The shunt impedance as defined in Eq. 3.22 is dependent on the cavity geometry and on the power losses determined by the cavity surface. We can then define 
the normalized shunt impedance (usually called $R$ over $Q$ ), which is independent on the material properties (power dissipation), as:

$$
\frac{R}{Q}=\frac{\left(E_{a c c}\right)^{2}}{P_{d} / d^{2}} \frac{1}{Q_{0}}=\frac{\left(E_{a c c}\right)^{2}}{\omega U / d^{2}} .
$$

Such parameter defines the efficiency of acceleration per unit of stored energy and length for a fixed value of frequency. The $R / Q$ value for a nine-cells TESLA type cavity is $581 \Omega[15]$.

\subsection{Radio-Frequency Measurements}

In order to characterize the properties of superconducting resonators, rf tests are performed in a cryogenic environment. Such kind of tests are generally called vertical tests, since the cavity is hanged vertically inside a cryostat filled up with liquid helium. The temperature can be easily decreased by pumping on the helium bath, reducing the pressure in equilibrium with the liquid phase. By pumping on the helium bath all the temperatures in the range $4.2 \mathrm{~K}$ to about $1.4 \mathrm{~K}$ are reachable.

The rf test is usually performed by measuring the cavity performance- Qfactor versus accelerating field $E_{a c c}$-at $2 \mathrm{~K}$ and at $1.5 \mathrm{~K}$. Usual normal-conducting copper resonators can be easily tested by meaning of a network analyzer since their Q-factor is usually of the order of $10^{4}-10^{5}$ (depending on frequency, temperature and resistivity). Such Q-factor magnitudes are small enough that the full width at half maximum of the resonance peak is larger than the network analyzer bandwidth, so that the resonance peak can be easily resolved.

Superconducting cavities must instead be measured by means of a more com-

plicated and indirect measurement system since their quality factor is usually of the order of $10^{9}-10^{11}$ (depending on frequency and temperature), and therefore not measurable by a network analyzer.

The basic idea underneath a vertical rf test is to excite the electromagnetic 
resonant field inside the resonator through a coaxial antenna (input coupler) fed by an external rf source. In order to collect a signal from the cavity, another antenna (pickup) is placed in the other side of the cavity. With a power balance argumentation, the cavity quality factor and accelerating field may be calculated by considering the powers leaking from and reflected by the resonator.

Hence, during the rf test the variables we need to fully characterize the cavity performance are three: the forward power fed by the rf system $\left(P_{f}\right)$, the reflected power from the resonator $\left(P_{r}\right)$ and the transmitted power leaking through the pick$\operatorname{up}\left(P_{t}\right)$.

3.2.1 Power Balance of Cavity plus Antennae. As previously discussed in Subsection 3.1.4, when the electromagnetic field is left to decay in the resonator without the presence of any antenna, the stored energy decreases exponentially with a decay time $\tau_{0}$ dependent only on the cavity intrinsic dissipation mechanisms (Eq. 3.17). In the same way, when antennae are used to couple the electromagnetic field in the cavity, the field will decay exponentially, but with decay time dependent also on the amount of power leaking out the resonator though the antennae. We can then define the loaded quality factor as:

$$
Q_{L}=\tau_{L} \omega
$$

where $\tau_{L}$ is the decay time of cavity plus antennae.

From the cavity point of view, the total power dissipated is:

$$
P_{\text {tot }}=P_{d}+P_{c}+P_{t}
$$

where $P_{c}$ is the power dissipated on the input coupler.

We can therefore rewrite the loaded quality factor $Q_{L}$ in terms of total dissi- 
pated power as:

$$
\frac{1}{Q_{L}}=\frac{P_{t o t}}{\omega U}=\frac{P_{d}+P_{c}+P_{t}}{\omega U}=\frac{1}{Q_{0}}+\frac{1}{Q_{c}}+\frac{1}{Q_{t}},
$$

with $Q_{c}$ and $Q_{t}$ the quality factor of coupler and pick-up respectively.

At this point, the loaded quality factor can be redefined in a more handy way by introducing the two quantities $\beta$ and $\beta_{t}$, respectively the coupler and pick-up coupling parameters. $Q_{L}$ is then defined as:

$$
\frac{1}{Q_{L}}=\frac{1}{Q_{0}}\left(1+\beta+\beta_{t}\right) \approx \frac{1}{Q_{0}}(1+\beta),
$$

where $\beta=Q_{0} / Q_{c}$ and $\beta_{t}=Q_{0} / Q_{t}$. Usually the pick-up antenna is chosen to be weakly coupled $\left(Q_{t} \gg Q_{0}\right)$, and $\beta_{t} \ll 1$, meaning that the power dissipated in the pick-up can be neglected since very small.

Once that the coupling parameter $\beta$, forward, reflected and transmitted power are known, the cavity behavior can be fully described by meaning of the following power balance:

$$
P_{f}=P_{d}+P_{r}+P_{t}
$$

where $P_{f}, P_{r}$ and $P_{t}$ are the only variables measurable during the rf test.

We should now introduce an important parameter called reflection coefficient $\Gamma[13,68]$, which defines the reflection probability of a signal with a certain frequency $\Omega$ fed to the resonator:

$$
\Gamma=\frac{\beta-1-i Q_{0} \delta}{\beta+1+i Q_{0} \delta}
$$

where $\delta=\Omega / \omega+\omega / \Omega$.

From Eq. 3.29 we can directly define the reflected power $P_{r}$ and the power leaking through the input coupler inside the cavity $P_{\text {in }}$ as:

$$
P_{r}=P_{f}|\Gamma|^{2} \quad ; \quad P_{\text {in }}=P_{f}-P_{r}=P_{f}\left(1-|\Gamma|^{2}\right)
$$


As defined in [13], the power leaking inside the resonator $P_{\text {in }}$ may also be expressed as:

$$
P_{i n}=\sqrt{\frac{4 P_{f} \omega U}{Q_{c}}-\frac{\omega U}{Q_{c}}},
$$

therefore by meaning of Eqs. 3.29, 3.30 and 3.31 when $\Omega=\omega$, the reflected power may be rewritten as:

$$
P_{r}=\left(\sqrt{P_{c}}-\sqrt{P_{f}}\right)^{2},
$$

where $P_{c}=\omega U / Q_{c}$ is the power leaking out from the input coupler. The reflected power should then be imagined as the superposition of two signals: the direct reflection of the forward power signal and the signal emitted from the cavity through the input coupler.

3.2.2 Vertical Test of a Superconducting Resonator. The characterization of the resonator behavior is carried out by measuring the quality factor as a function of the accelerating field when this latter is fully immersed in liquid helium inside a vertical cryostat. Every rf test is always divided in two different steps, the decay measurement needed to calculate the decay time of the pick-up probe $\tau_{t}$, and the continuous-wave (CW) measurement during which we take advantage of $\tau_{t}$ to fully characterize the cavity behavior.

3.2.2.1 Decay Measurement. The first step needed to properly characterize the cavity is to determine the coupling strength between the cavity and the input antenna. In order to do that the cavity is fed with a forward power signal modulated with a square pulse. The reflected signal observed from the oscilloscope tell us the coupling condition: under coupled $\beta<1$, critically coupled $\beta=1$ and over coupled $\beta>1$. In Figure 3.5 an example of reflected signal read by means of an oscilloscope is reported.

In all three coupling conditions, when the power is switched on $(t=0)$ the 


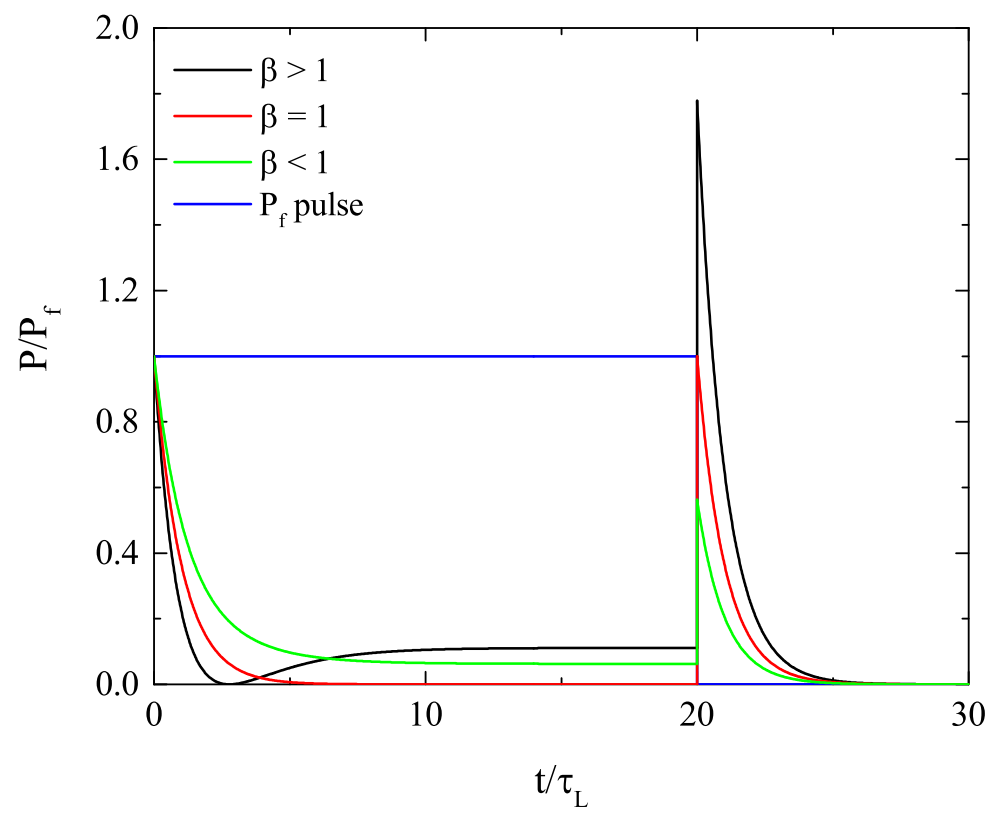

Figure 3.5. Examples of reflected power signal as a function of time in conditions of under, critically and over coupling. The forward power pulse is shown in blue.

reflected signal presents a peak with maximum equal to the power level of the square pulse. When the pulse ends and the forward signal goes to zero, the reflected power exhibits a second peak which height depends on the coupling condition. If $\beta>1$ the second peak is higher that the first one, if $\beta=1$ the two peaks have same height, while if $\beta<1$ the second peak is lower than the first one.

The coupling parameter $\beta$ can then be calculated rearranging Eq. 3.29:

$$
\beta=\frac{1 \pm \sqrt{P_{r} / P_{f}}}{1 \mp \sqrt{P_{r} / P_{f}}},
$$

where $P_{r}$ and $P_{f}$ are the forward and reflected powers measured in steady state. The upper sign is used for $\beta>1$, while the lower for $\beta<1$.

When the forward power pulse ends, the transmitted power decays with same time constant $\tau_{L}$ of the stored energy:

$$
P_{t}(t) \propto U(t) \propto e^{-t / \tau_{L}},
$$

hence by fitting the $P_{t}$ signal decay, the time constant can be extrapolated and the 
loaded quality factor $Q_{L}$ is known: $Q_{L}=\omega \tau_{L}$.

At this point, the intrinsic quality factor of the cavity $Q_{0}$ can be calculated by meaning of Eq. 3.27:

$$
Q_{0}=Q_{L}(1-\beta)
$$

Since $\beta_{t}=P_{t} / P_{d}=Q_{0} / Q_{t}$ and $Q_{t}=\omega \tau_{t}$, the decay time associated to the power dissipation on the pick-up probe is then defined as:

$$
\tau_{t}=\frac{Q_{0}}{\omega \beta_{t}}=\frac{Q_{0}\left(P_{f}-P_{t}-P_{t}\right)}{\omega P_{t}} \quad ; \quad P_{d}=P_{f}-P_{r}-P_{t}
$$

with $P_{f}, P_{r}$ and $P_{t}$ measured during the steady state of the square pulse.

Usually, several calculations of $\tau_{t}$ are performed in order to obtain a more accurate and precise value.

3.2.2.2 Continuous-Wave Measurement. Now that the pick-up probe decay time $\tau_{t}$ is known, the calculation of intrinsic quality factor $Q_{0}$ and accelerating field $E_{a c c}$ can be performed straightforwardly.

Since the pick-up quality factor is defined as $Q_{t}=\omega U / P_{t}=\omega \tau_{t}$, the stored energy in the resonator as a function of the transmitted power can be defined as: $U=\tau_{t} P_{t}$. Therefore, taking advantage of Eq. 3.28 the intrinsic quality factor is:

$$
Q_{0}=\frac{\omega U}{P_{d}}=\frac{\omega \tau_{t} P_{t}}{P_{f}-P_{r}-P_{t}},
$$

where $P_{f}, P_{r}$ and $P_{t}$ are the powers directly measured point by point during the rf test.

The accelerating field in the cavity can instead be directly calculated by rearranging Eq. 3.23:

$$
E_{a c c}=\frac{1}{d} \sqrt{\frac{R}{Q} \omega U}=\frac{1}{d} \sqrt{\frac{R}{Q} \omega \tau_{t} P_{t}},
$$

where $R / Q$ and $d$ are known form the cavity geometry. 
3.2.3 Measurement System. The rf test of a resonator is performed by means of a complex rf measurement system able to acquire the values of forward, reflected and transmitted power. A simplified schematics of a rf system is reported in Figure 3.6a.

The cavity is fed with a rf drive signal provided by a system able to track the cavity frequency, by means of a phase-locked loop (PLL) that controls a voltage controlled oscillator (VCO).

When configured in PLL mode, the VCO provides a periodic low power signal with frequency controlled by an input voltage. A portion of the VCO's output signal is first directed through a phase shifter, needed to adjust the phase of the signal from the VCO, and then to a rf mixer. In the rf mixer, the signal form the VCO is mixed with a portion of the transmitted signal from the cavity. The mixer output is then a voltage proportional to the phase difference between the two input signals, which is used to control the VCO's output frequency. With such PLL arrangement the cavity frequency can be tracked accurately.

This kind of PLL rf sources are largely adopted in the measurement of critically and nearly critically coupled cavities, where the resonance peak is very sharp (bandwidth of the order of $1 \mathrm{~Hz}$ ) and the loaded quality factor. When the cavity instead operates in the cryomodule, because of the high power coupler $\beta$ is too large to get an accurate and precise measurement of the quality factor. The intrinsic quality factor $Q_{0}$ can be measured only calorimetrically, monitoring the helium consumption due to the resonator.

The second portion of the rf drive signal coming from the VCO-PLL enters in a high power amplifier, and is fed to the cavity through an input line ending with the input coupler antenna. Right before the cavity, along the input line, a bidirectional coupler is placed. With this device it is possible to read directly the forward power 
a)

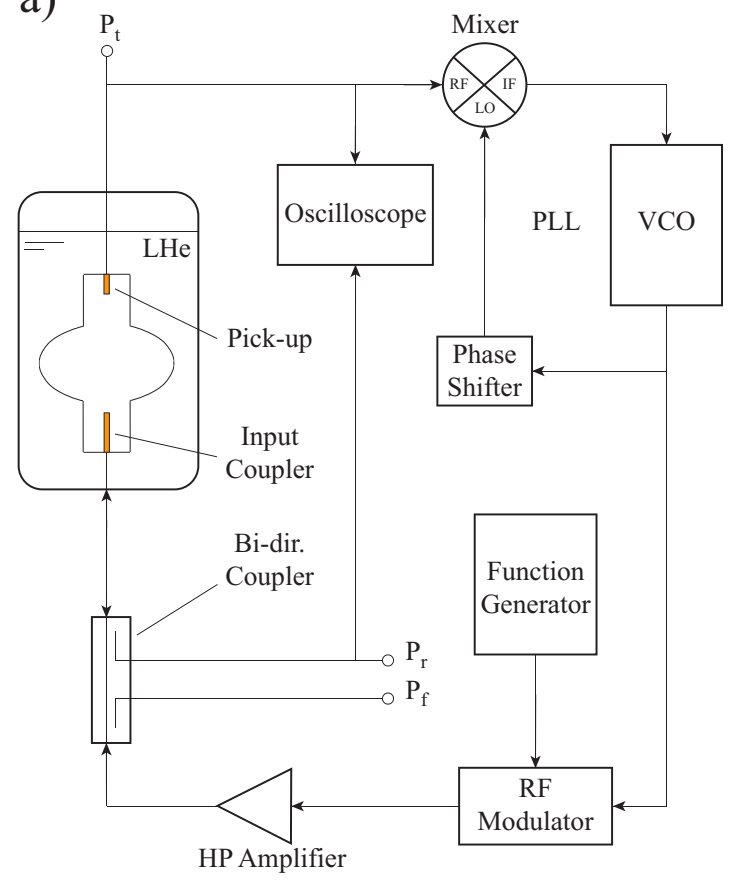

b)
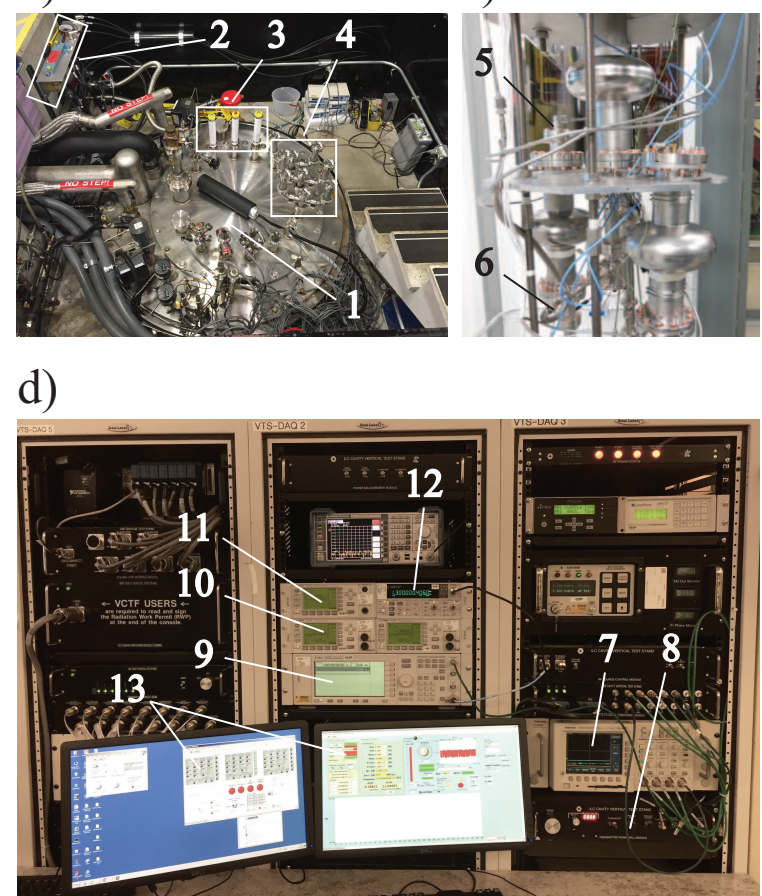

Figure 3.6. In (a) a simplified schematic of the rf system is reported, while in (b), (c) and (d) the top plate of the Dewar "VTS1", the vertical test stand and the rf measurement system at the Fermilab vertical test facility respectively are shown.

$\left(P_{f}\right)$ coming from the amplifier, and the reflected power from the cavity $\left(P_{r}\right)$, by means two power meters.

At the opposite side of the cavity, the pick-up probe antenna is placed. The transmitted signal leaking though the pick-up is split in two portions. The first one goes straight to the third power meter $\left(P_{t}\right)$, while the second one becomes the transmitted power input for the PLL.

Generally, an oscilloscope is connected in order to monitor transmitted and reflected power (forward power is usually monitored as well).

During the decay measurement, a function generator and a rf modulator are used in order to modulate the rf drive signal from the VCO into square power pulses before entering in the amplifier. 
In Figure 3.6b the top plate of the "VTS1" Dewar at the Fermilab vertical test facility is shown (1) where 2 corresponds to the bidirectional coupler, while 3 and 4 are the forward/reflected and transmitted signal feedthroughs connected respectively to the input and pick-up antennae e through two rf cables.

An example of single cells cavities attached to the stand to be inserted in the Dewar are reported in Figure 3.6c. Indicated in figure the pick-up antenna and input power coupler with numbers 5 and 6 respectively.

The Fermilab vertical test facility's rf system racks are shown in Figure 3.6d, where in sequence from 7 to 13 are shown: the oscilloscope, the PLL box with manual phase shifter, the VCO, the transmitted signal power meter, the forward and reflected signals power meter, the on-line frequency counter (it shows the frequency as tracked by the PLL) and the control program.

3.2.4 System Calibration. Before starting the rf measurement of the cavity the system must be properly calibrated. Indeed, the three powers $P_{f}, P_{r}$ and $P_{t}$ are measured out of the Dewar, which means that these are not the correct powers values at the cavity. Appropriate attenuation factor must be calculated in order to correct such measured variables.

The calibration procedure described in this subsection is implemented at the Fermilab vertical test facility, and therefore not applicable to every rf system. Anyway, the philosophy underneath every single step is analogous from system to system. In general the calibration for a single cell cavity is composed of five steps:

\section{- Reflected warm cable attenuation}

A calibrated rf source (with $P_{s} \simeq 30 \mathrm{~mW}$ ) is connected at the feedthrough $\mathrm{C}$ (see Figure 3.7a) and the power is measured at the connection B by means of the rf system power meter $\left(P_{r}\right)$. The attenuation of the warm segment $\mathrm{BC}$ is 
a)

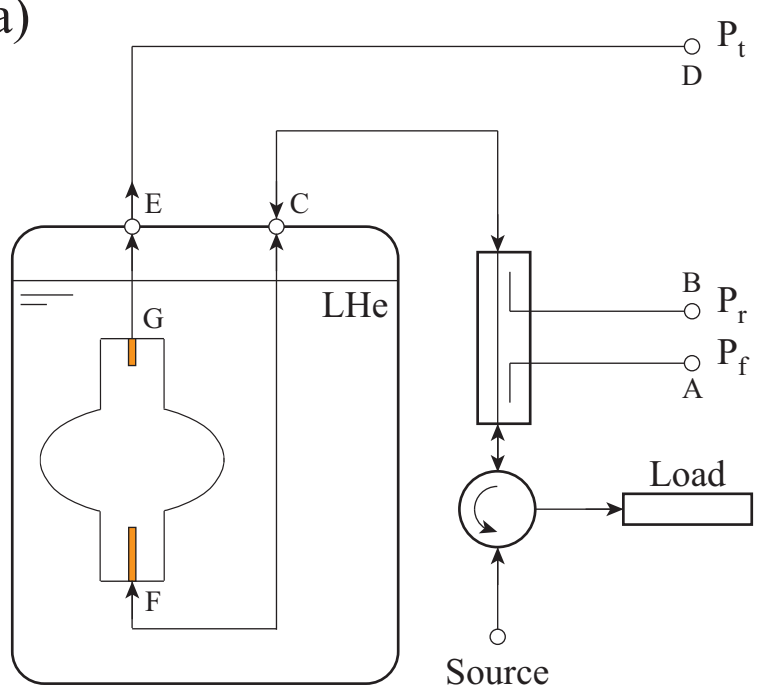

b)

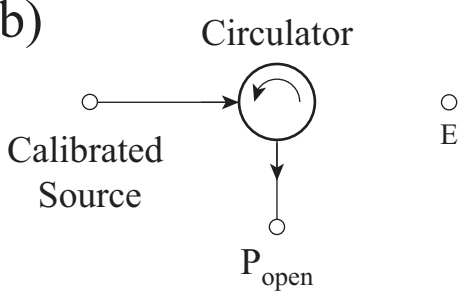

c)

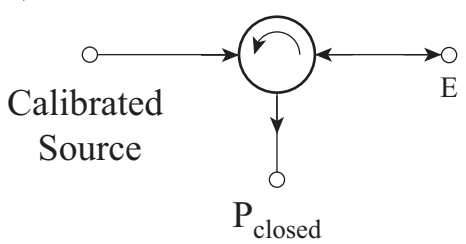

Figure 3.7. Schematics of the cables and connections needed to perform the rf system calibration.

then calculated as:

$$
C_{B C}[\mathrm{~dB}]=10 \log _{10}\left(\frac{P_{s}}{P_{r}}\right)
$$

- Transmitted warm cable attenuation

The same calibrated $\mathrm{rf}$ source (with $P_{s} \simeq 30 \mathrm{~mW}$ ) is connected at the feedthrough E (see Figure 3.7a) and the power is measured at the connection D by means of the rf system power meter $\left(P_{t}\right)$. The attenuation of the warm segment DE is then calculated as:

$$
C_{D E}[\mathrm{~dB}]=10 \log _{10}\left(\frac{P_{s}}{P_{t}}\right)
$$

- Forward warm cable attenuation

In this case the power is fed by the rf system's source (see Figure 3.7a), and the power is measured at the connection $\mathrm{A}\left(P_{f}\right)$ and at the connection $\mathrm{C}\left(P_{\text {cyostat }}\right)$ with a portable power meter. The attenuation of the warm segment $\mathrm{AC}$ is the equal to:

$$
C_{A C}[\mathrm{~dB}]=10 \log _{10}\left(\frac{P_{f}}{P_{\text {cryostat }}}\right)
$$




\section{- Transmitted cold cable attenuation}

In this case the attenuation is calculated by measuring the reflected signal from the cavity. Since at this stage the cavity is already superconductive, any signal sent from the connection E to G (see Figure 3.7a), that does not fall inside the cavity resonance bandwidth (about $1 \mathrm{~Hz}$ ), will be totally reflected back.

By means of a circulator connected to the calibrated rf source, as shown in Figure $3.7 \mathrm{~b}$, we can measure the attenuation of the circulator alone $C_{\text {open }}$ as:

$$
C_{\text {open }}[\mathrm{dB}]=10 \log _{10}\left(\frac{P_{s}}{P_{\text {open }}}\right)
$$

At this point, following the schematics in Figure 3.7c, the circulator is connected to the feedthrough E at the Dewar's top plate. Then by measuring $P_{\text {closed }}$ we can calculate the attenuation of the circulator plus the cold section EG as:

$$
C_{\text {closed }}[\mathrm{dB}]=10 \log _{10}\left(\frac{P_{s}}{P_{\text {closed }}}\right)
$$

By subtracting $C_{\text {oped }}$ from $C_{\text {closed }}$, the attenuation of the cold section EG is:

$$
C_{E G}[\mathrm{~dB}]=\frac{C_{\text {closed }}-C_{\text {open }}}{2}=5 \log _{10}\left(\frac{P_{\text {open }}}{P_{\text {closed }}}\right),
$$

where the factor $1 / 2$ accounts for the double distance traveled by the signal (since reflected, the signal travels two times the segment EG).

\section{- Forward/reflected cold cable attenuation}

Now that the attenuation of all segments except for the segment CF (see Figure 3.7a) is known, we just need to measure $P_{f}$ at the connection A and $P_{r}$ at the connection B when some power is fed to the cavity by the rf system's source, in order to measure the last attenuation. As done before, we measure the reflected signal from the cavity, therefore we must make sure that the cavity is not tracked in frequency by the PLL and that the rf system is out of resonance. Once $P_{f}$ and $P_{r}$ are known they should be converted into dBm $(P[\mathrm{dBm}]=$ 
$\left.10 \log _{10}(P / 0.001)\right)$ and the power conservation equation may be written as:

$$
\left(P_{f}[\mathrm{dBm}]-C_{A C}-C_{C F}\right)-\left(P_{r}[\mathrm{dBm}]+C_{B C}+C_{C F}\right)=0
$$

from which the attenuation of the cold section $\mathrm{CF}$ is calculated as:

$$
C_{C F}[\mathrm{~dB}]=\frac{1}{2}\left[\left(P_{f}[\mathrm{dBm}]-C_{A C}\right)-\left(P_{r}[\mathrm{dBm}]+C_{B C}\right)\right]
$$

Now that all the cable sections' attenuation are known, the three calibration coefficients for forward $C_{f}$, reflected $C_{r}$ and transmitted $C_{t}$ powers can be calculated:

$$
\begin{aligned}
& C_{f}[\mathrm{~dB}]=-C_{A C}-C_{C F} \quad \rightarrow \quad C_{f}=10^{\left(-C_{A C}-C_{C F}\right) / 10}, \\
& C_{r}[\mathrm{~dB}]=C_{B C}+C_{C F} \quad \rightarrow \quad C_{r}=10^{\left(C_{B C}+C_{C F}\right) / 10}, \\
& C_{t}[\mathrm{~dB}]=C_{D E}+C_{E G} \quad \rightarrow \quad C_{t}=10^{\left(C_{D E}+C_{E G}\right) / 10} .
\end{aligned}
$$

The dimensionless coefficients (not in $\mathrm{dB}$ ) can be now multiplied to the forward, reflected and transmitted powers measured, in order to account for the attenuation generated by the rf system cables.

3.2.5 Cavities Instrumentation. Other than the necessary antennae needed to excite and probe the field in the resonator, also other instrumentation is usually implemented to carry out the experiments.

One of the most useful measurement systems is the temperature mapping system [69] (T-map). The T-map is based on an array of carbon resistive sensors placed on a total of 36 boards - 16 per board - with boards positioned every $10^{\circ}$ around the cavity circumference. In Figure 3.8a a cavity equipped with the T-map system is shown, while in Figure 3.8b a T-map board with 16 thermometers is shown. The T-map is often used in order to visualize how the dissipation is distributed on the cavity surface, and it is also very useful in order to visualize the cavity quench location. 

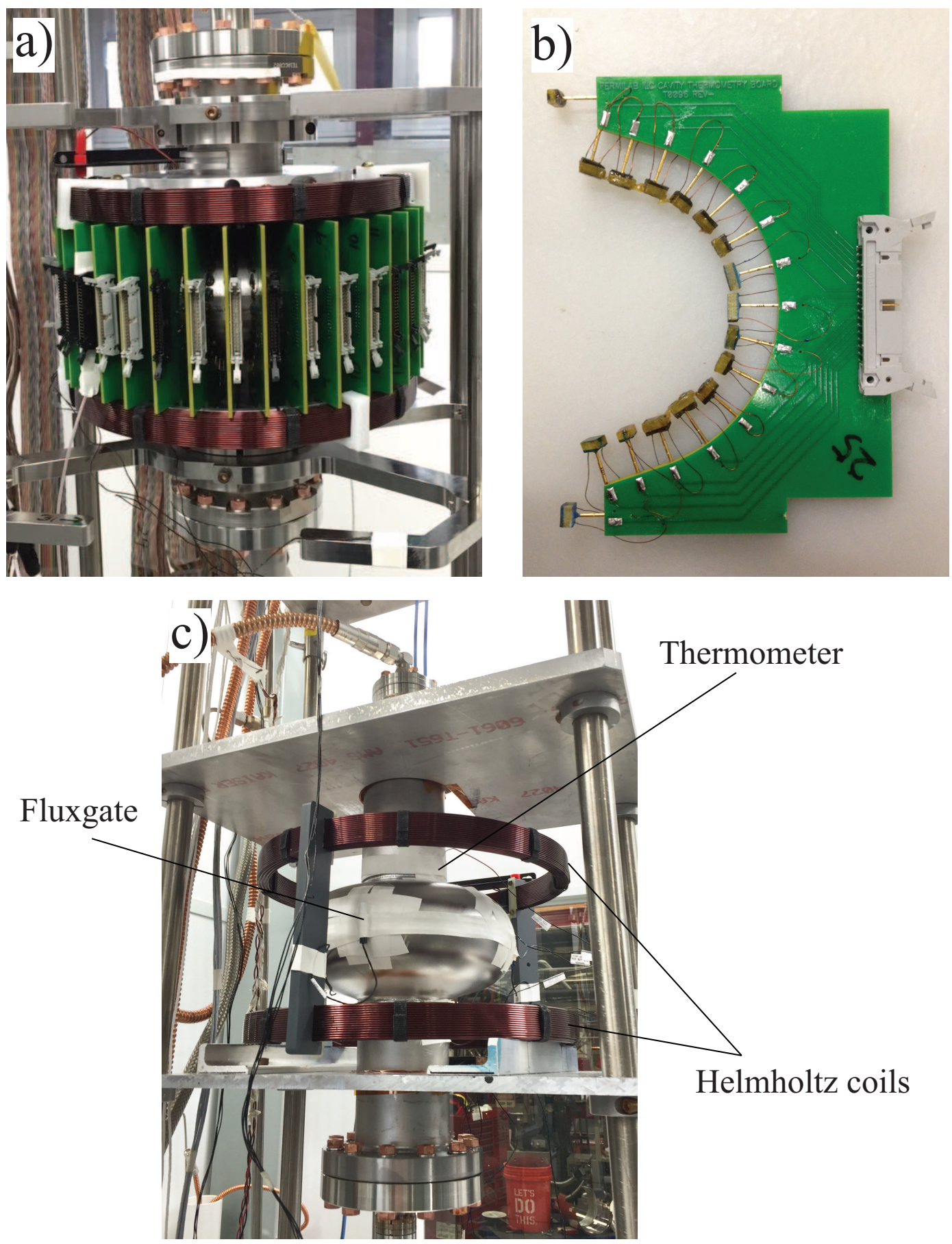

Figure 3.8. In (a) TESLA type cavity equipped with T-map and Helmholtz coils, in (b) T-map board in detail and in (c) TESLA type cavity equipped with thermometers, fluxgates and Helmholtz coils. 
In order to apply uniform magnetic fields around the cavity, Helmholtz coils are used. In Figure 3.8a and c, two different configurations of cavity instrumentation with Helmholtz coils (with and without T-map) are shown. The magnetic field applied to the cavity is monitored with single-axis Bartington Mag-01H cryogenic fluxgate magnetometers. Usually, 4 fluxgates are placed around the cavity equator circumference every $90^{\circ}$, with axis parallel to the cavity beam pipe, as shown in Figure 3.8c. The temperature of the cavity is instead monitored with carbon thermometers usually placed at the two irises and at the equator, for a total of four.

\subsection{Superconducting Accelerating Cavities Performance}

Superconducting radio-frequency elliptical cavities are usually produced from bulk niobium sheets that are deep-drawn to form half cells then connected together with an electron beam welder. Just after the production, cavities undergo to several surface treatments in order to maximize their performance before being assembled in an accelerator.

In general, the performance in terms of quality factor and accelerating field is defined by the final surface treatments the cavity have received. The first step common to all the treatments is the bulk electro-polishing (EP), which is performed just after the cavity is fully welded (flanges included), and with which 100-200 $\mu \mathrm{m}$ are removed from the cavity internal surface. The second common step is the $800^{\circ} \mathrm{C}$ baking for $3 \mathrm{~h}$ in vacuum. Such step is performed to get rid of hydrogen absorbed during the chemistry, to relax stresses introduced by the fabrication process and to partially recrystallize the material.

After these two standard processes, the cavity surface can be treated with different surface treatments dependently on the type of performance needed. Four standard and one recently discovered surface treatments now-a-days exist. 
- Electro-Polishing (EP): the cavity is simply electro-polished in order to remove $10-20 \mu \mathrm{m}$ of material from the internal surface. The EP process is generally performed at lower temperature than the bulk EP in order reduce the hydrogen contamination. The solution used is composed by hydrofluoric and sulfuric acids usually with ratio $1: 10$.

Such cavities always show the so-called high-field-Q-slope (HFQS), i.e. the rapid increase of the intrinsic residual resistance with the accelerating field above a certain field threshold, consistently found to be around $25 \mathrm{MV} / \mathrm{m}$. The HFQS might be explained as the progressive break-down of the proximity coupling of nano-hydrides precipitates at the cavity surface [54].

- Buffer Chemical Polishing (BCP): the inner cavity's surface is chemically etched with the BCP solution in order to remove 10-20 $\mu \mathrm{m}$ of material. The BCP solution is composed by hydrofluoric, nitric and phosphoric acids with usual ratio $1: 1: 2$.

Generally, BCP cavities shows comparable performance to EP cavities, with HFQS field threshold usually at lower values (about $20 \mathrm{MV} / \mathrm{m}$ ). The HFQS might be explained with the analog arguments used for EP cavities.

- $120{ }^{\circ} \mathrm{C}$ baking: the first step just after the baking is to electro-polish the rf surface in order to remove $40 \mu \mathrm{m}$ of material. The cavity is then assembled for the vertical test and evacuated. Once ready to be tested the cavity is baked at $120{ }^{\circ} \mathrm{C}$ for $48 \mathrm{~h}$ in situ.

$120^{\circ} \mathrm{C}$ baked cavities can reach higher gradients than EP and BCP cavities and does not present any HFQS. Such mild baking is indeed considered the "cure" to HFQS, but how it affects the material properties in order to eliminate the HFQS is still source of discussion in the community. Studies [70] suggests that the "cure" to the HFQS is associated to the diffusion of oxygen in the bulk form the 


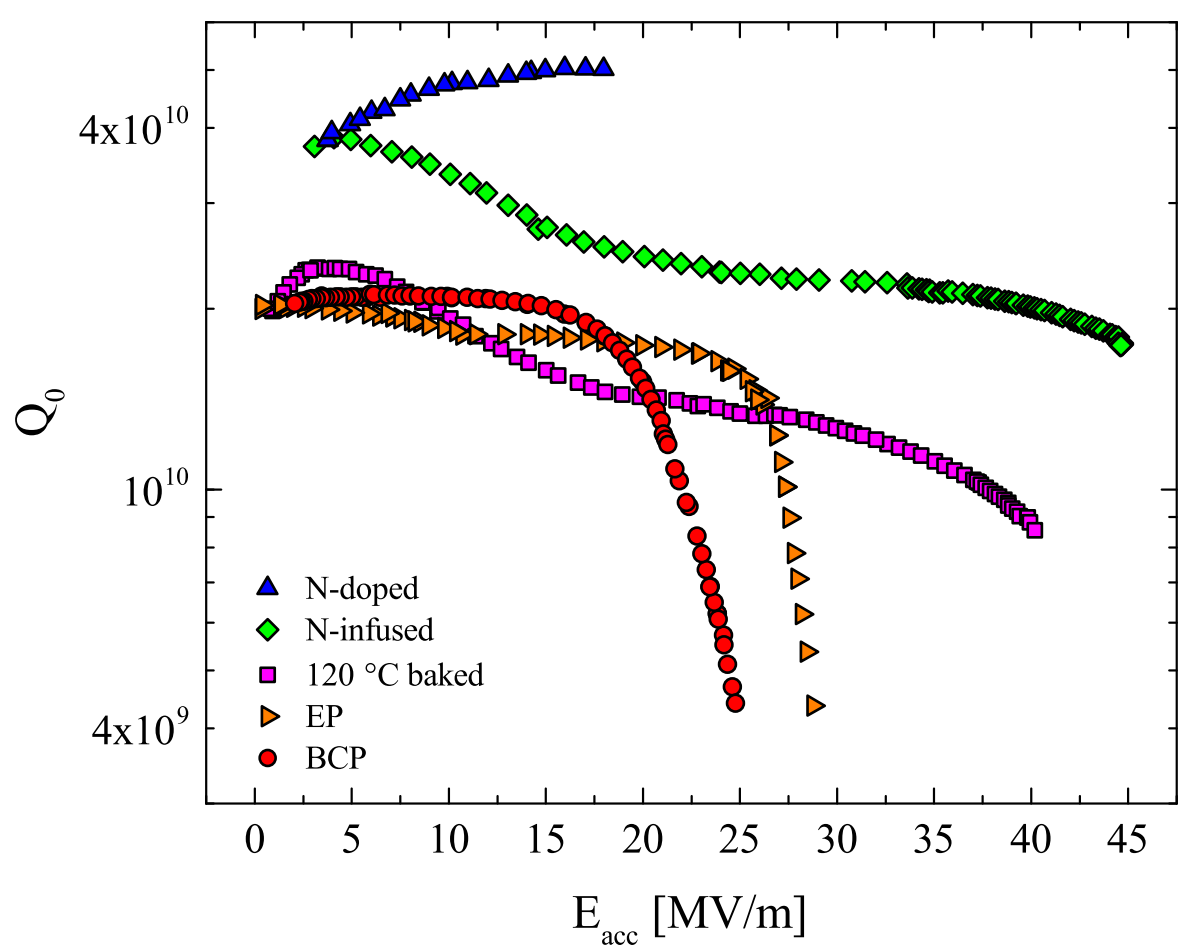

Figure 3.9. Example of cavities performance for different surface finishing.

native oxide at the niobium surface. The more exhaustive description though, in my personal opinion, is discussed in [71]. The vacuum baking at $120{ }^{\circ} \mathrm{C}$ is shown to introduce vacancies in the near-surface layer, which bound hydrogen and prevent its precipitation in nano-hydrides that generate the HFQS.

Anyway, the must interesting effect of the $120{ }^{\circ} \mathrm{C}$ baking for the purpose of this thesis is the generation of a high $\kappa$ layer at the rf surface that can help to enhance the accelerating gradient of such cavities, as explained in Chapter 6. Also, such cavities were demonstrated in [10], and theoretically described in Chapter 4 , to possess low vortex surface resistance.

- N-doping: after the cavity is baked $3 \mathrm{~h}$ at $800{ }^{\circ} \mathrm{C}$ the temperature in the furnace is typically maintained constant and nitrogen is inlet at the constant pressure of 25 mTorr. Nitrogen is then left in the furnace for a certain amount of time (minutes). After the nitrogen supply is closed, the cavity can (optional step) 
be left in the furnace to anneal for a couple of minutes more and then the heating is stopped. After the thermal treatment, the cavity is electro-polished to remove the first 5-10 $\mu \mathrm{m}$ of material from the inner surface in order to get rid of spurious niobium nitride phases.

$\mathrm{N}$-doped cavities show the highest quality factor ever measured for bulk niobium TESLA cavities [72], and therefore enable the possibility of operating continuous-wave machines with TESLA cavities such as LCLS-II. The main drawbacks of such technology are the higher flux-related surface resistance per amount of magnetic field trapped, as described in Chapter 4, and the lower quench field than EP, BCP or $120^{\circ} \mathrm{C}$ baked cavities, as discussed in Chapter 5 .

- N-infusion: after the standard $800{ }^{\circ} \mathrm{C}$ bake, the temperature in the furnace is lowered between $120-160{ }^{\circ} \mathrm{C}$ without breaking the vacuum, nitrogen is inlet at the constant pressure of $25 \mathrm{mTorr}$ and the process let to continue for $48 \mathrm{~h}$ or more. After the thermal treatment no chemistry is done.

Cavities prepared with this new surface treatment show high accelerating gradients and Q-factors about two times larger than standard $120{ }^{\circ} \mathrm{C}$ baked cavities. This new technology allows for high Q-factors at high fields with the promise to cut the cryogenic cost for high energy linear machines like the ILC. As explained in Chapter 6, also this thermal treatment (like the $120^{\circ} \mathrm{C}$ baking) generates a dirty layer at the surface that enhances the accelerating gradient.

In Figure 3.9 some examples of typical performance for EP, BCP, $120^{\circ} \mathrm{C}$ baked, $\mathrm{N}$-doped and $\mathrm{N}$-infused cavities are reported. 


\section{CHAPTER 4}

\section{VORTEX SURFACE IMPEDANCE}

\subsection{Chapter Overview}

As introduced in Chapter 2, when the superconductive transition is performed in presence of external magnetic field magnetic flux can be trapped in superconducting materials as energetically stable flux quanta in the mixed state of type II superconductors, or as magnetic flux pinned at defects in the Meissner state of type I and type II superconductors. In some circumstances, magnetic flux lines can penetrate in the Meissner state without needing of pinning sites and, as a consequence of the demagnetization effect, exist in the so-called intermediate state. Independently on their nature, any trapped magnetic flux structure can introduce dissipation in both de and rf domains $[2,33,73,74]$.

Controlling the pinning force of the superconducting material, it is possible to eliminate the vortex dc dissipation, enabling superconductors to transport very high currents, without any dissipation, up to the depinning current. Exceeded the depinning current, the dissipation due to vortices motion takes place because of the viscous drag force [44].

On the other hand, in rf applications the vortex dissipation cannot be avoided most of the times. Even if pinned, the vortex flux line dissipates power because of the oscillation induced by the rf currents. Therefore, the trapped flux problem is of critical importance for superconducting accelerating cavities, especially when high quality factors are needed for their implementation in continuous wave accelerators.

With the discovery of the nitrogen doping treatment [72], unprecedented high quality factors (Q-factors) are achievable in SRF niobium cavities. The presence of nitrogen as interstitial impurity in Nb allows for high Q-factors by decreasing the tem- 
perature dependent part of the surface impedance [49]. On the other hand, N-doped cavities show higher dissipation per unit of magnetic field trapped than standard cavities. It was indeed observed $[10,75,76]$, that the vortex-related resistance per unit of trapped magnetic field is function of the electron mean free path, and therefore of the cavity thermal history. $\mathrm{N}$-doped cavities operating at $1.3 \mathrm{GHz}$ fall exactly in the mean free path region where the vortex dissipation is increased.

Two different approaches to describe the flux motion exist. The first one assumes a point-like description of the vortex [77, 78, 79, 80, 81], where the pinning force is usually introduced as an elastic restoring force independent on the position of the pinning point and distance from it.

The second approach assumes the flux line as a bi-dimensional object that possess a certain tension [73]. Usually when the latter description is assumed, the pinning force is disregarded $[46,82]$ and the vortex response calculated in absence of pinning.

If both vortex line tension and pinning force are considered, the analytic form of the latter must possess the dependence on the distance from the rf surface, complicating substantially the problem. A clever way to partially overcome the problem is achieved by introducing Dirichlet boundary conditions at the pinning site, as described in [42]. The main drawback though is the impossibility of describing the problem as a function of the pinning strength, since as experimentally observed [74], it is one of the most important parameters to characterize the vortex dynamics in a superconductor.

In this chapter a different approach is proposed. The motion equation that describes the vortex displacement is defined in such a way to be different for every point of the vortex flux line and hence dependent on the distance $z$ from the rf surface. 
To some extent, this approach is similar to the point-like description of the vortex response, but it differs from it substantially in terms of pinning force description. Differently than the point-like description, where the vortex is extremely rigid and remains straight through the entire material thickness, this approach describes the motion of a flexible mono-dimensional vortex line.

The most noticeable point of this new approach is its simplicity and excellent description of the experimental data acquired for SRF cavities $[10,75,76]$.

Introducing a clear dependence of the pinning force on the electron mean free path $l$, the experimentally observed bell-shaped trend of the trapped flux surface resistance as a function of the mean free path $[10,75,76]$ can be explained as the interplay of the resistivity calculated in flux flow and pinning regimes.

Since vortices are usually multiple-pinned in the material, the model takes care also of situations where more than one pinning point per vortex are present. Indeed, the position of the pinning point, their number and their strength are of extreme importance in order to fully describe the experimental data.

It is also demonstrated that the transition between pinning and flux flow regimes may be obtained not only by crossing the depinning frequency [77], but also by tuning the mean free path value of the superconducting material.

\subsection{Single Vortex Resistivity}

When trapped at the rf surface, the magnetic flux experiences a force generated by the interaction with the Eddy currents induced by the oscillating rf field. The rf current density $\mathbf{j}(t)$ exercises a force on the magnetic flux quantum $\phi_{0}$ in the vortex, accordingly to the Lorentz force. The magnetic force acting on a single vortex per unit of length $F_{L}$ is:

$$
F_{L}=\left|\mathbf{j} \times \phi_{0} \hat{n}\right|=j_{0} \phi_{0} \sin \theta e^{i \omega t-z / \lambda}
$$


where $j_{0}$ is the rf current, $\theta$ the angle between $\mathbf{j}$ and the normal to the $\mathrm{rf}$ surface $\hat{n}$, $\omega$ the rf angular frequency and $\lambda=\lambda_{0} \sqrt{1+\left(\xi_{0} / l\right)}$, with $\lambda_{0}$ the penetration depth (London penetration depth) and $\xi_{0}$ the coherence length.

We can write the motion equation of a single vortex subjected to the Lorentz force as follows:

$$
M \ddot{x}(t)=F_{L}+F_{v}+F_{p},
$$

with $M$ being the inertial mass of the vortex per unit of length as defined by $\mathrm{J}$. Bardeen and M. J. Stephen [36]:

$$
M=\frac{2 \pi^{2} n_{n} m \xi_{0}^{4} B_{c 2}(T)}{\phi_{0}} \sin ^{2} \alpha,
$$

where $B_{c 2}\left(=B_{c 2}(0)\left[1-\left(T / T_{c}\right)^{2}\right]\right)$ is upper critical field, $\alpha$ the Hall angle with respect the normal to $\mathbf{j}$ defined by [36]:

$$
\tan \alpha=\frac{e \phi_{0} \tau}{\pi m \xi_{0}^{2}}
$$

$e$ and $m$ are the charge and mass of the electron respectively, and $\tau=l / v_{f}$ the Drude's electron relaxation time, with $v_{f}$ the Fermi velocity.

The other forces acting on the vortex are $F_{p}$ the pinning force and $F_{v}$ the viscous drag force. In this description the Magnus force (see Section 2.4) is neglected, as well as the interaction between vortices [33], since we assume $H \ll H_{c 2}$.

The viscous drag force is defined as $F_{v}=-\eta \dot{x}$ where $\eta$ is the vortex drag coefficient per unit of length. For the purpose of this model the J. Bardeen and M. J. Stephen [36] definition is adopted, since the area of interest is in the limit $T \ll T_{c}$. The viscous drag coefficient per unit of length is then defined as:

$$
\eta(l, T)=\frac{\phi_{0} B_{c 2}(T)}{\rho_{n}},
$$

where $\rho_{n}(l)\left(=\left(n_{n} e^{2} \tau / m\right)^{-1}\right)$ is the normal-electrons resistivity. 
The pinning force description is extremely complicated and related to the nature of the pinning sites $[33,83]$. In the present model no discrimination between different types of pinning site is done, thus an idealistic description of the pinning potential is assumed.

The ideal mono-dimensional pinning potential is function of the effective coherence length $\xi\left(=\left(1 / \xi_{0}+1 / l\right)^{-1}\right)$ and is described by an inverse Lorentzian function [84]. Since the vortex is a linear object, the pinning site is localized at a certain distance $q$ from the rf surface. The associated pinning potential has centroid at such pinning position and approaches zero in every direction far from it.

By adopting a bi-dimensional Lorentzian potential and by limiting the pinning interaction along the oscillation direction $(\hat{x})$, we are able to simplify the problem significantly. The analytic form for the pinning force is maximum at the pinning site, and varies as a function of the $z$ direction. Because of that, the vortex line experiences a local pinning force different for every $z$ location, resulting in a displacement of the vortex line that is function of the distance from the rf surface.

Since the motion equation to describe the vortex oscillation is solved analytically, this last must be transformed in order to be linear in $x$ by expanding the pinning potential to the second order with respect to $x$ :

$$
\begin{aligned}
U_{p}(x, z, l) & =-\sum_{i=0}^{n} \frac{U_{0_{i}} \xi^{2}}{\xi^{2}+x^{2}+\left(z-q_{i}\right)^{2}} \\
& \approx-\sum_{i=0}^{n} \frac{U_{0_{i}} \xi^{2}}{\xi^{2}+\left(z-q_{i}\right)^{2}}+\sum_{i=0}^{n} \frac{U_{0_{i}} \xi^{2}}{\left[\xi^{2}+\left(z-q_{i}\right)^{2}\right]^{2}} x^{2}
\end{aligned}
$$

where $U_{0_{i}}$ is the potential depth per unit of length of the $i$-th pinning point, while the sum accounts for multiple pinning potentials centered at the $q_{i}$-th positions acting on the same vortex.

A single pinning potential is then parabolic along the oscillation direction 
a)

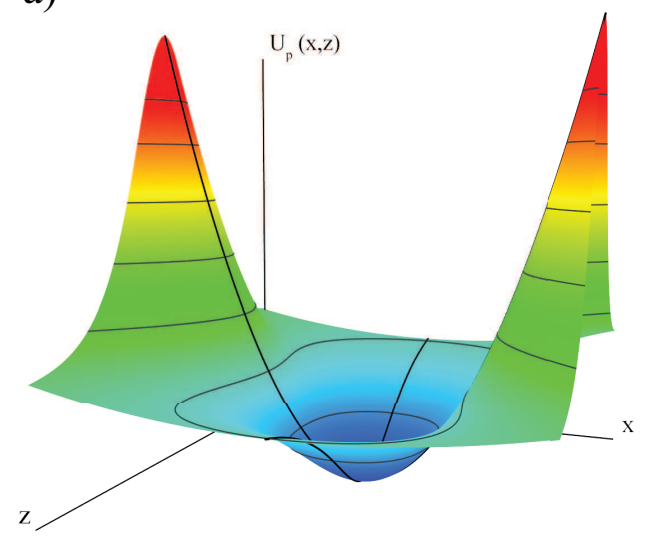

b)

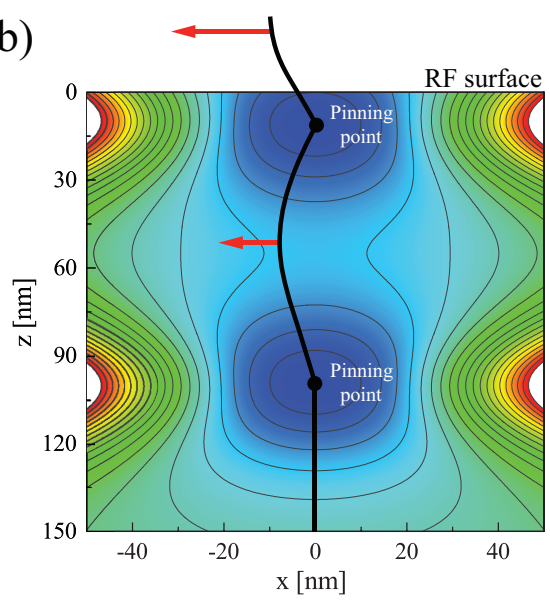

Figure 4.1. In (a) tri-dimensional representation of the pinning potential considered. In (b) contour plot of two pinning potential acting on the fame flux line.

and Lorentzian along $\hat{z}$. The tri-dimensional representation of the pinning potential considered is plotted in Figure 4.1a, while in Figure 4.1b an example of an oscillating flux line subjected to two potentials in series is shown.

The pinning force per unit of length is then defined as:

$$
\begin{aligned}
F_{p}(x, z, l) & =-\frac{\partial}{\partial x} U_{p} \\
& =-\sum_{i=0}^{n} \frac{2 U_{0_{i}} \xi^{2}}{\left(\xi^{2}+\left(z-q_{i}\right)^{2}\right)^{2}} x \\
& =-p(z, l) x,
\end{aligned}
$$

with pinning constant $p(z, l)$.

Substituting Eqs. 4.1, 4.7 and the viscous drag force with $\eta$ equal to Eq. 4.5 in Eq. 4.2, we get the motion equation of a single vortex:

$$
\ddot{x}(z, t, l)+\alpha \dot{x}(z, t, l)+\beta^{2} x(z, t, l)=\gamma e^{i \omega t},
$$

which corresponds to a driven-dumped oscillator second order differential equation, with $\alpha=\eta / M, \beta^{2}=p / M, \gamma=j \phi_{0} \sin \theta / M$ and $j=j_{0} e^{-z / \lambda}$.

The solution of such differential equation is:

$$
x(z, t, l)=\left(A_{1}+i A_{2}\right) e^{i \omega t},
$$


where:

$$
\begin{aligned}
& A_{1}(z, l)=\frac{j \phi_{0} \sin \theta\left(p-M \omega^{2}\right)}{\left(p-M \omega^{2}\right)^{2}+(\eta \omega)^{2}} \\
& A_{2}(z, l)=-\frac{j \phi_{0} \sin \theta \eta \omega}{\left(p-M \omega^{2}\right)^{2}+(\eta \omega)^{2}} .
\end{aligned}
$$

We can now calculate the average (active) dissipated power $\langle P\rangle$ and the reactive power $\langle Q\rangle$ per unit of volume as:

$$
\begin{aligned}
& \langle P(z, l)\rangle=\frac{1}{T} \int_{0}^{T} \operatorname{Re}\left\{\mathcal{F}_{L}(t) \dot{x}(z, t, l)\right\} d t \\
& \langle Q(z, l)\rangle=\frac{1}{T} \int_{0}^{T} \operatorname{Im}\left\{\mathcal{F}_{L}(t) \dot{x}\left(z, t-\frac{T}{4}, l\right)\right\} d t
\end{aligned}
$$

where $T$ is the $\operatorname{rf}$ period $(T=2 \pi / \omega)$ and $\mathcal{F}_{L}=j B_{v} \sin \theta$ is the Lorentz force per unit of volume, with $B_{v}$ the vortex magnetic field.

Solving the two integrals for the real and imaginary parts of Lorentz force and vortex velocity $\dot{x}(z, t, l)$, we obtain the apparent power per unit of volume:

$$
\begin{aligned}
\langle S(z, l)\rangle & =\langle P\rangle+i\langle Q\rangle \\
& =\frac{\omega \phi_{0} B_{v} \sin ^{2} \theta}{2\left[\left(p-M \omega^{2}\right)^{2}+(\eta \omega)^{2}\right]}\left[\eta \omega+i\left(p-M \omega^{2}\right)\right] j^{2} \\
& =\frac{1}{2} \rho j^{2}
\end{aligned}
$$

where $\rho$ is the complex vortex resistivity.

Since $B_{v}=\phi_{0} / \pi \xi_{0}^{2}$, where $\pi \xi_{0}^{2}$ is the approximated vortex core area, the complex vortex resistivity $\rho$ is then equal to:

$$
\begin{aligned}
\rho(z, l) & =\rho_{1}+i \rho_{2} \\
& =\frac{\omega \phi_{0}^{2} \sin ^{2} \theta}{\pi \xi_{0}^{2}\left[\left(p-M \omega^{2}\right)^{2}+(\eta \omega)^{2}\right]}\left[\eta \omega+i\left(p-M \omega^{2}\right)\right],
\end{aligned}
$$

where $\rho_{1}$ and $\rho_{2}$ describe respectively the resistive and reactive behavior of the vortex when subjected to a rf current. 
4.2.1 Flux Flow Regime. In the large mean free path limit the vortex dissipation is described by the flux flow regime. In such limit we can neglect the pinning force $(p \sim$ 0) since the pinning potential becomes very shallow driven by the larger coherence length.

On the other hand, the viscous coefficient (Eq. 4.5) is larger because of its dependence on the normal-state resistivity (the larger $l$, the smaller $\rho_{n}$ and the larger $\eta)$. Therefore, the main force acting on the vortex is the viscous drag force. Since very small compared to $\eta$ the vortex inertial mass can be neglected as well.

Neglecting inertial and pinning terms, we can rewrite the motion equation (Eq. 4.8) as:

$$
\alpha \dot{x}(z, t, l)=\gamma e^{i \omega t}
$$

This first order differential equation can be easily solved by meaning of the ansatz used above (Eq. 4.9). Solving the equation, we obtain an imaginary coefficient:

$$
A_{2}(l)=-\frac{j \phi_{0} \sin \theta}{\omega \eta} .
$$

Calculating the apparent power in Eq. 4.12, we get a purely real resistivity.

$$
\rho_{1}(l)=\frac{\phi_{0}^{2} \sin ^{2} \theta}{\pi \xi_{0}^{2} \eta} .
$$

Such definition of the flux flow resistivity is equivalent to the result obtained by Y. B. Kim, et al. [44] and by R. Marcon, et al. [79] (real part). We should also notice that the same form of $\rho_{1}$ can be obtained by neglecting the term $\left(p-M \omega^{2}\right)^{2}$ in Eq. 4.13.

This result suggests that the vortex dissipation for large mean free path values is independent on the frequency and depends only on the mean free path. 
Moreover, as the purity of the material increases ( $l$ increases) the resistivity decreases since inversely proportional to the viscous coefficient $\eta$. For mean free path values big enough, the resistivity is minimized, and the dissipation introduced by the vortex oscillation negligible.

4.2.2 Pinning Regime. In the limit of small mean free path values, we can define the pinning regime. Since $\eta$ decreases with decreasing $l$, the viscous drag force is negligible in the small $l$ limit. On the contrary, the pinning force is larger due to a small coherence length which increases the steepness of the pinning potential. In this regime the main force acting on the vortex is the pinning force, and the vortex inertial mass may also be neglected.

Considering the complex resisitivity defined in Eq. 4.13, we can obtain a form of $\rho$ that describes the vortex impedance in the pinning regime for intermediate values of mean free path. As already discussed, for small $l$ values the dominant contribution is the pinning force. Therefore, neglecting the inertial term $M \omega^{2}$ and rewriting the denominator as $\pi \xi_{0}^{2} p^{2}$, we can define the complex resistivity as:

$$
\rho(z, l)=\frac{\omega \phi_{0}^{2} \sin ^{2} \theta}{\pi \xi_{0}^{2} p^{2}}[\eta \omega+i p] .
$$

In such an intermediate regime, the vortex response is both of resisitive and reactive nature. In any case, as the mean free path decreases the real part decreases with it ( $\eta$ decreases) till it becomes negligible. Such a limiting condition is well described by the vortex motion equation, where both the inertial and viscous contributions are neglected:

$$
\beta^{2} x(z, t, l)=\gamma e^{i \omega t}
$$

Solving for $x$ and comparing with the ansatz in Eq. 4.9, we obtain a pure real coefficient:

$$
A_{1}(z, l)=\frac{j \phi_{0} \sin \theta}{p}
$$


and the apparent power in Eq. 4.12 is totally reactive. Thus, we get the pure reactive response of the vortex oscillation:

$$
\rho_{2}(z, l)=\frac{\omega \phi_{0}^{2} \sin ^{2} \theta}{\pi \xi_{0}^{2} p} .
$$

Such a result suggests that for low mean free paths a vortex interacting with an oscillating field does not contributes to active power dissipation, its response is purely reactive. Moreover, in the limit of very small mean free paths the pinning constant $p$ increases substantially and it minimizes the reactive response as well.

\subsection{Multiple Vortices Surface Impedance}

Now that the vortex resistivity is known, the surface impedance for a single vortex is calculated assuming the classic definition:

$$
\begin{aligned}
Z_{1}(l) & =\frac{E_{x}(0)}{\int_{0}^{\infty} j_{x}(z) d z} \\
& =\left[\int_{0}^{\infty} \frac{e^{-z / \lambda}}{\rho(z, l)} d z\right]^{-1},
\end{aligned}
$$

where $j_{x}(z)=j_{x}(0) e^{-z / \lambda}$.

By means of the local description of a vortex (Section 2.3), when a finite value of magnetic field $(B)$ is applied to the superconductor during the transition, $N$ vortices are created, and each of them carries a magnetic flux quantum $\phi_{0}$ through an area $\pi \xi_{0}^{2}$, i.e. $N \phi_{0}=A B$, where $A$ is the normal conducting area that experiences the magnetic field at the transition.

We can therefore extend the single vortex resistance defined in Eq. 4.21 to a multi-vortex resistance multiplying by the fraction of area occupied by the trapped vortices $N \pi \xi_{0}^{2} / A=\pi \xi_{0}^{2} B / \phi_{0}$.

Now, we should also consider that most likely there will be a certain distribution of pinning potentials in the material. In order to take into account that, we 
define the probability density of finding the pinning point at the position $q_{i}$ as $\Gamma\left(q_{i}\right)$, and the probability density that a pinning potential has strength $U_{0_{i}}$ as $\Lambda\left(U_{0_{i}}\right)$.

Both the two probability densities are described by a normalized Gaussian distribution. The $\Gamma\left(q_{i}\right)$ distribution has variance $\sigma_{q_{i}}$ and centroid $q_{i}^{0}$ :

$$
\begin{aligned}
& \Gamma\left(q_{i}\right)=B_{i} e^{-\frac{\left(q_{i}-q_{0_{i}}\right)^{2}}{2 \sigma_{q_{i}}^{2}}}, \\
& B_{i}=\frac{\sqrt{\frac{2}{\pi \sigma_{q_{i}}^{2}}}}{\operatorname{Erf}\left(\frac{q_{i}^{\vee}-q_{i}^{0}}{\sqrt{2} \sigma_{q_{i}}}\right)+\operatorname{Erf}\left(\frac{q_{i}^{0}}{\sqrt{2} \sigma_{q_{i}}}\right)},
\end{aligned}
$$

with $q_{i}^{\vee}$ the maximum extension of the integration domain over all the possible positions $q_{i}$, defined as $q_{0_{i}}+5 \sigma_{q_{i}}$. While the distribution $\Lambda\left(U_{0_{i}}\right)$ has variance $\sigma_{U_{0_{i}}}$ and centroid $U_{0_{i}}^{0}$ :

$$
\begin{gathered}
\Lambda\left(U_{0_{i}}\right)=C_{i} e^{-\frac{\left(U_{0_{i}}-U_{0_{i}}^{0}\right)^{2}}{2 \sigma_{U_{0_{i}}}^{2}}}, \\
C_{i}=\frac{\sqrt{\frac{2}{\pi \sigma_{U_{0_{i}}}^{2}}}}{\operatorname{Erf}\left(\frac{U_{0_{i}}^{\vee}-U_{0_{i}}^{0}}{\sqrt{2} \sigma_{U_{0_{i}}}}\right)-\operatorname{Erf}\left(\frac{U_{0_{i}}^{\wedge}-U_{0_{i}}^{0}}{\sqrt{2} \sigma_{U_{0_{i}}}}\right)},
\end{gathered}
$$

where $U_{0_{i}}^{\vee}$ and $U_{0_{i}}^{\wedge}$ are the extremes of the integration domain, set as $U_{0_{i}}^{0} \pm 5 \sigma_{U_{0_{i}}}$. If $U_{0_{i}}^{0}-5 \sigma_{U_{0_{i}}}<0$, then $U_{0_{i}}^{\wedge}=0$, since no negative values are allowed.

The vortices surface impedance weighted over pinning point position and strength distributions, for a given trapped field $B$ is defined as:

$$
Z(l)=\frac{\pi \xi_{0}^{2} B}{\phi_{0}} \int_{0}^{q_{0}^{\vee}} \int_{U_{0_{0}}}^{U_{0_{0}}^{\vee}} \cdots \int_{0}^{U_{0}^{\vee}} \int_{U_{0_{n}}^{\wedge}}^{q_{n}^{\vee}} \frac{\prod_{i=0}^{n}\left[\Gamma\left(q_{i}\right) \Lambda\left(U_{0_{i}}\right)\right]}{\int_{0}^{L} \frac{e^{-z / \lambda}}{\rho(z)} d z} d U_{0_{0}} d q_{0} \cdots d U_{0_{n}} d q_{n}
$$

with $L$ being the cavity wall thickness. 


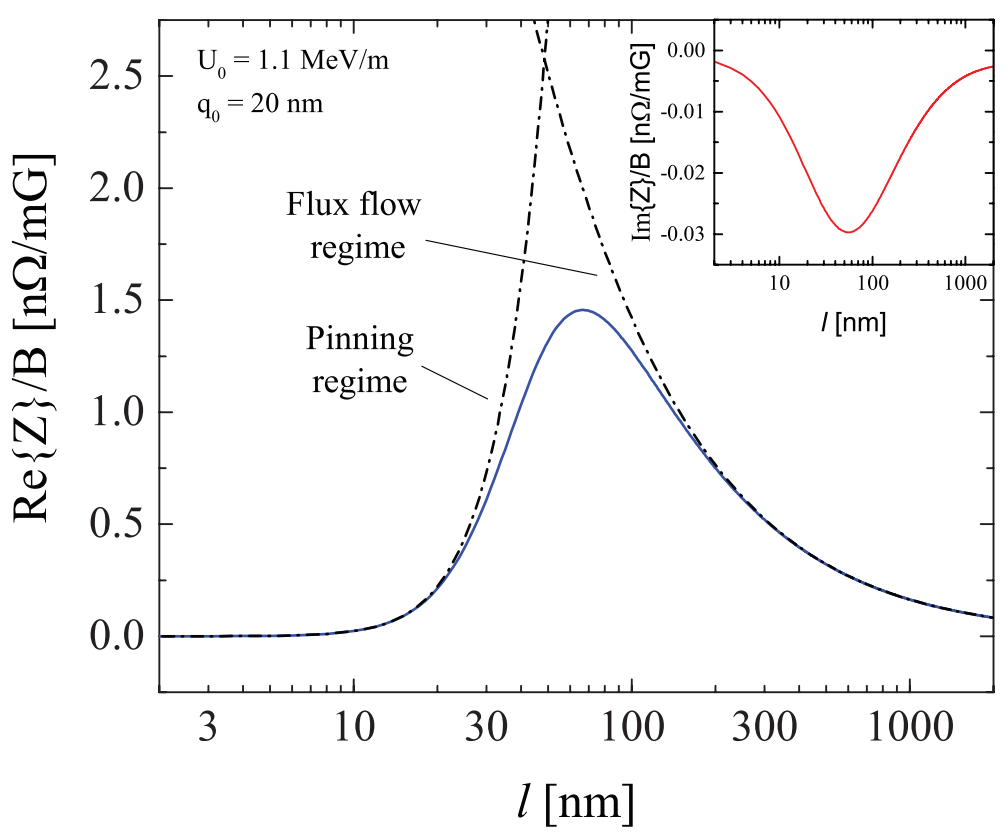

Figure 4.2. Real part of the surface impedance as a function of the mean free path. The dashed-dotted lines correspond to the pinning and flux flow regimes, for small and large mean free path values respectively. In the inset the imaginary part of surface impedance is plotted as a function of the mean free path.

In order to simplify the interpretation of the simulation results, we adopt a Dirac- $\delta$ distribution for both $\Gamma\left(q_{i}\right)$ and $\Lambda\left(U_{0_{i}}\right)$-instead of the more realistic Gaussian probability function - and a single pinning point with $q_{0}=20 \mathrm{~nm}$ and $U_{0_{0}}^{0} \equiv U_{0}=$ 1.1 $\mathrm{MeV} / \mathrm{m}$, in all the simulation performed (if not differently specified). All the other parameters used are reported in Table 4.1.

In Figure 4.2, the real part of the vortices surface impedance normalized to the trapped flux $B$ as a function of the electron mean free path $l$ is plotted. In the same plot the two limits for clean and dirty material (flux flow and pinning regimes), calculated with Eqs. 4.16 and 4.17 respectively are also shown.

In the inset of Figure 4.2, the imaginary part of the surface impedance is reported. Its behavior is roughly opposite to the real part, but its absolute value negligible with respect to it, since about two orders of magnitude lower. 
Table 4.1. Parameters used in the simulations for niobium.

\begin{tabular}{lll}
\hline \hline Parameter & Value & Reference \\
\hline$\xi_{0}$ & $38 \cdot 10^{-9} \mathrm{~m}$ & B. W. Maxfield, et al.[85] \\
$\lambda_{0}$ & $39 \cdot 10^{-9} \mathrm{~m}$ & B. W. Maxfield, et al. $[85]$ \\
$B_{c 2}(0)$ & $442 \mathrm{mT}$ & S. J. Williamson, et al. [86] \\
$v_{f}$ & $1.37 \cdot 10^{6} \mathrm{~m} \mathrm{~s}^{-1}$ & N. W. Ashcroft, et al. $[87]$ \\
$n$ & $5.56 \cdot 10^{28} \mathrm{~m}^{-3}$ & N. W. Ashcroft, et al. $[87]$ \\
$f$ & $1.3 \cdot 10^{9} \mathrm{~Hz}$ & \\
$T$ & $1.5 \mathrm{~K}$ & \\
$L$ & $3 \mathrm{~mm}$ & \\
\hline
\end{tabular}

The most noticeable feature showed in Figure 4.2 is the presence of a peak in the surface resistance around $70 \mathrm{~nm}$. For large mean free paths the surface resistance follows perfectly the flux flow result and decreases with the cleanliness of the material.

On the opposite, when the the mean free path decreases the surface resistance deviates substantially from the flux flow regime, approaching the pinning regime.

Starting in the large mean free path region (flux flow regime) and moving towards small mean free path values (pinning regime), both the pinning constant $p$ and the viscous drag coefficient $\eta$ are subjected to a substantial variation. In particular, $p$ increases driven by the decreased coherence length, while $\eta$ decreases because of the lower normal-state conductivity.

The decreasing of the surface resisitance in the pinning regime can be explained by the vanishing of the real part of the resistivity for small mean free path values, as discussed in Section 4.2.2. For very dirty materials, the drag coefficient can be neglected, and the vortices response to a rf field is purely reactive.

For large mean free paths instead, the situation is the opposite. As discussed in Section 4.2.1, for very large values of mean free path the resistivity is purely real, 


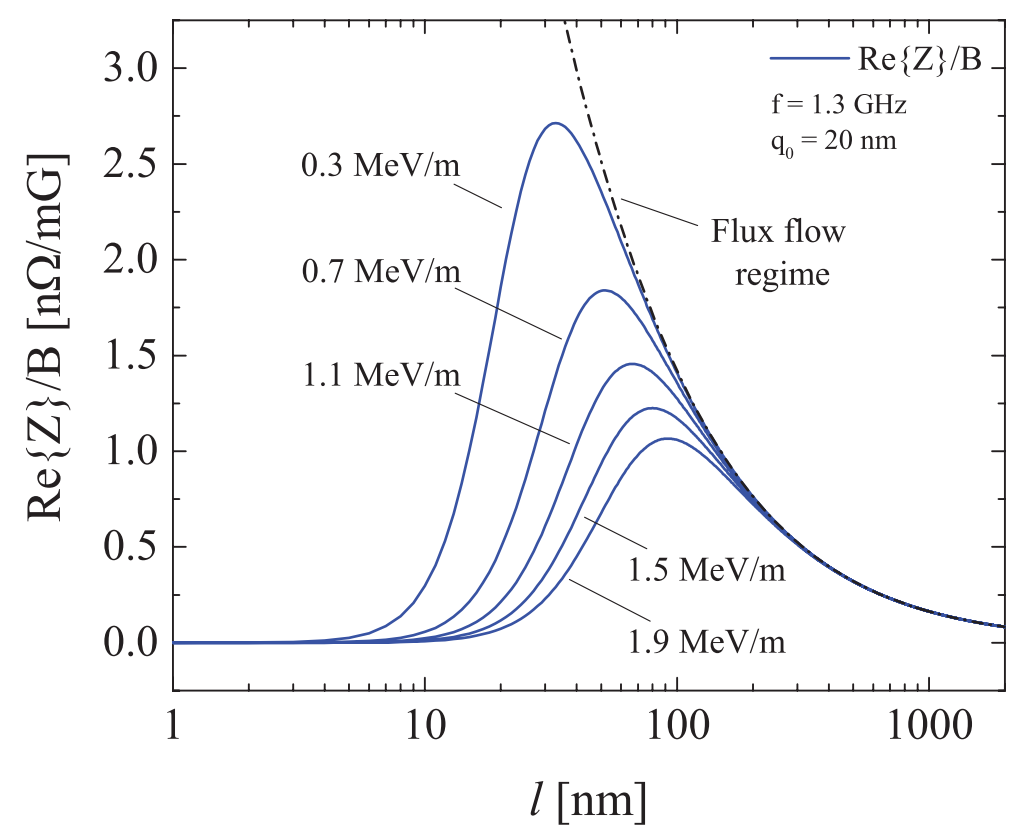

Figure 4.3. Real part of the surface impedance as a function of the mean free path for different values of $U_{0}$.

but the drag coefficient is so large that the vortices response is weak and the surface impedance tends to zero-less movable vortices dissipate less.

The peak is then generated by the interplay of flux flow and flux pinning regimes. In other words, we observe the progressing variation of the vortices response from purely reactive - low mean free paths, to purely resisitive-large mean free paths.

4.3.1 Pinning Potential Depth Dependence. The pinning potential depth $U_{0}$ is a parameter that modifies the pinning constant $p$, and therefore the pinning force (Eq. 4.7). In order to visualize the effect of different $U_{0}$ on the surface resistance, the real part of the surface impedance as a function of the mean free path for increasing values of pinning potential depth $U_{0}$ is plotted in Figure 4.3. As shown, the surface resistance peak is decreased in height and its position shifted to larger mean free path values for increasing values of $U_{0}$.

Since $p$ increases linearly with $U_{0}$, we expect that the pinning force becomes 
larger and larger for increasing $U_{0}$. Such variation affects the maximum of the surface resistance and shifts it towards larger mean free path values.

A larger pinning force implies a wider mean free path range within which the pinning regime is favorable than the flux flow regime. In such scenario, larger values of $l$ are needed to decrease the pinning force and make it negligible with respect the the viscous drag force. This different balance of the forces in play results in a higher mean free path onset for the flux flow regime, and the surface resistance peak is enhanced to higher values.

4.3.2 Multiple Pinning. Let us now analyze the situation in which multiple pinning points are present per single flux line. The real part of the vortices surface impedance (Eq. 4.24) was calculated considering again a Dirac- $\delta$ distribution profile, but considering one, two or three pinning points.

We should from now on consider that the vortex oscillation is extended beyond the rf layer, where the rf current is present. This means that the power absorption is dependent on the whole fraction of an oscillating vortex. The active power is dependent on the distance from the rf layer: in the approximation where no pinning points are present, vortex segments far from the rf surface absorb less power than those near by it-Eq. 4.12 is indeed dependent on z. In Figure 4.4 the active power per unit of volume normalized with respect to $j_{0}^{2}$ is reported for single, double, triple and no pinning. The power absorption is not constant allover the flux line since its oscillation is not rigid, the vortex oscillates as a flexible body and therefore its power dissipation will depend on the $z$ position with respect the pinning point.

The effect of a pinning point is equivalent to a constrain on the flux line oscillation in the material. If the pinning point is far from the $\mathrm{rf}$ surface $\left(q_{0} \gg \lambda\right)$, then the dissipation will reach its constant and maximum value since the oscillation 


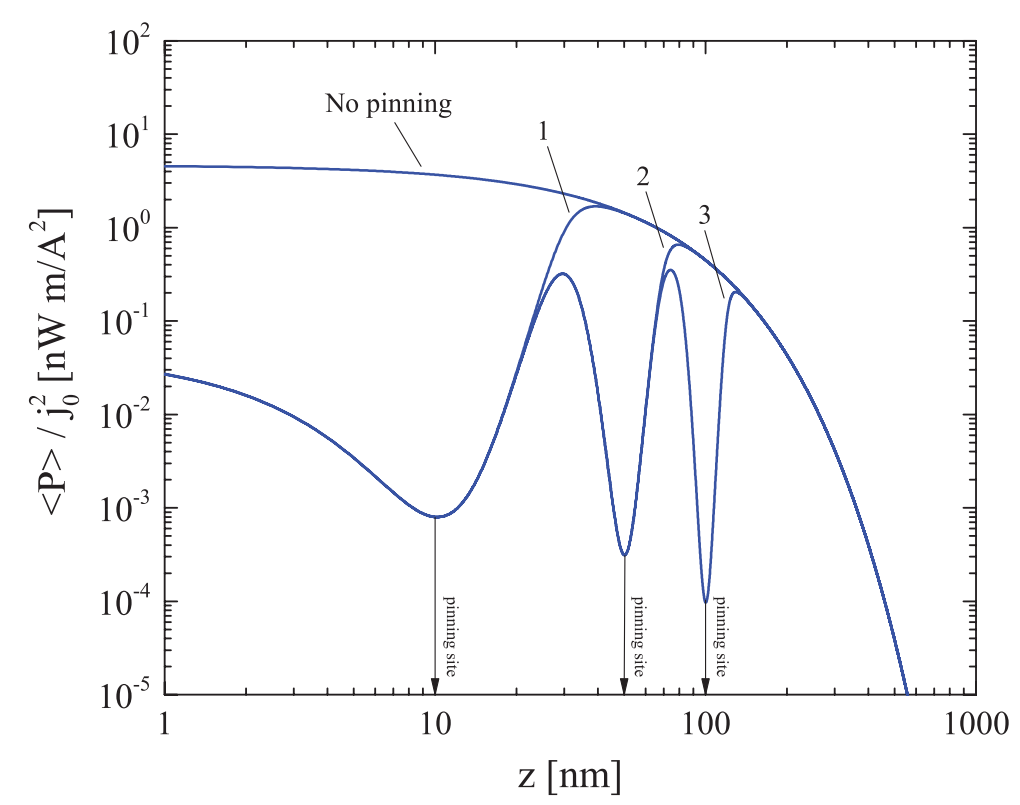

Figure 4.4. Simulation of the average power per unit of volume normalized to the current density squared for $l=10 \mathrm{~nm}$ and $U_{0}=1.1 \mathrm{MeV} / \mathrm{m}$. The four curves correspond respectively to no pinned vortex, single pinned vortex (curve 1 ), double pinned vortex (curve 2) and triple pinned vortex (curve 3). The three arrows shows the position of the pinning point.

is wider. Indeed, when the pinning point is far enough from the rf surface, the vortex oscillation is not perturbed by the presence of the pinning point, and the effect is equivalent to the condition when no pinning points are present at all.

In Figure 4.5a the surface resistance is plotted as a function of the distance of the pinning point from the surface $\left(q_{0}\right)$. Curve $a$ considers only one pinning point in the whole vortex line. The surface risistance is approximatevely constant for a single pinning point positioned 1 to $2 \mathrm{~nm}$ from the rf surface, it has a minimum for $q_{0}$ around $15 \mathrm{~nm}$ and it returns constant for $q_{0}>200 \mathrm{~nm}$.

If the pinning point is instead close to the rf surface, the oscillation will occur on both sides of the pinning point (as depicted in Figure 4.1b) and the position of the pinning point will define the magnitude of the dissipation. In Figure 4.5a the minimum of the surface resistance falls at about $15 \mathrm{~nm}$, which is roughly comparable 

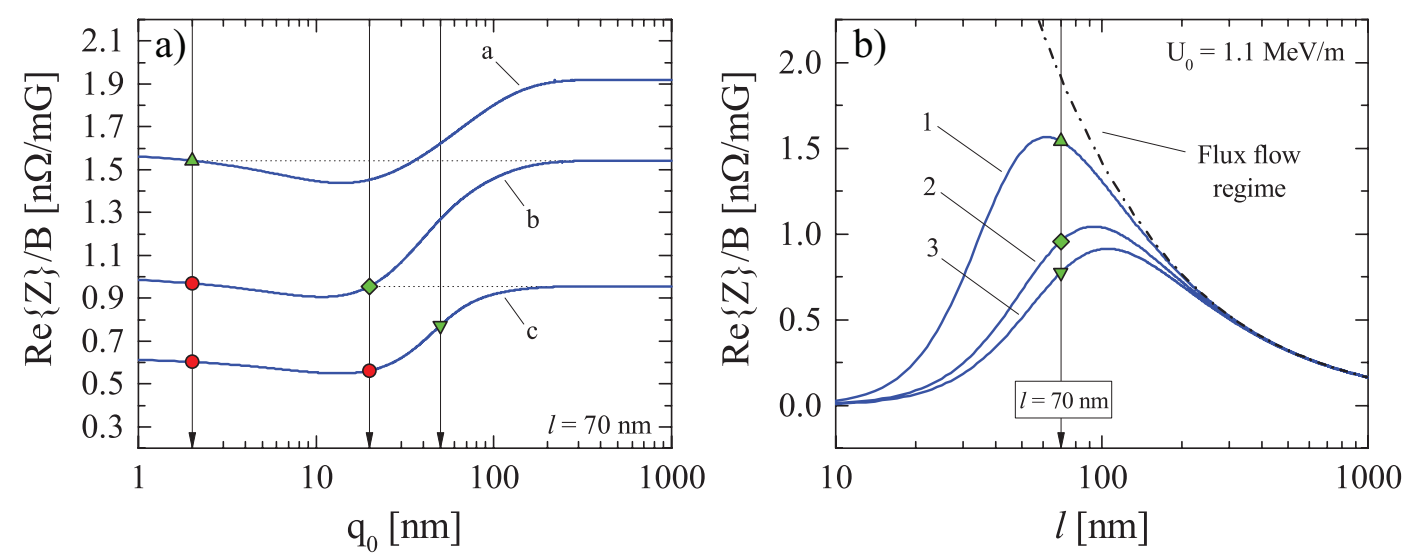

Figure 4.5. In (a) simulations of the surface resistance as a function of the pinning point position are reported. For all three the curves $l=70 \mathrm{~nm}$ and $U_{0}=1.1$ $\mathrm{MeV} / \mathrm{m}$. Curve $a$ considers only one variable pinning point position, curve $b$ one fixed and one variable pinning point positions and curve $c$ two fixed and one variable pinning point positions. In (b) the surface impedance as a function of the mean free path is reported in the condition of single (1), double (2) and triple (3) pinning per vortex flux line.

to half of the penetration dept for niobium with $l=70 \mathrm{~nm}(\lambda \sim 48 \mathrm{~nm})$. In such condition the vortex is well constrained and the resistance minimized, since both the two sides of the flux line (above and below the pinning point) have small oscillation amplitudes.

The picture of the dissipation due to the vortex oscillation can be better appreciated in Figure 4.4. When a pinning point is present (arrow) the dissipation is decreased because of the larger restoring force in that point. Far from the pinning point the vortex line has more freedom and the dissipation is larger.

Let us examine curve $b$ in Figure 4.5a. For such curve the coordinate $q_{0}$ corresponds to the position of the second pinning point, while the first one was assumed fixed at $q_{0_{1}}=2 \mathrm{~nm}$ from the surface (red dot on the curve).

The first noticeable effect is the overall lowering of the surface resistance for all the values of $q_{0}$. When $q_{0}$ increases above $10 \mathrm{~nm}$ the surface resistance increases and approaches its constant value for $q_{0}>200 \mathrm{~nm}$. 
Table 4.2. Pinning point positions of the green points in Figure 4.5. For all the points $U_{0}=1.1 \mathrm{MeV} / \mathrm{m}$ and $l=70 \mathrm{~nm}$.

\begin{tabular}{ll}
\hline \hline Point & Pinning points position \\
\hline$\Delta$ & $q_{0_{1}}=2 \mathrm{~nm}$ \\
$\diamond$ & $q_{0_{1}}=2 \mathrm{~nm}, q_{0_{2}}=20 \mathrm{~nm}$ \\
$\nabla$ & $q_{0_{1}}=2 \mathrm{~nm}, q_{0_{2}}=20 \mathrm{~nm}, q_{0_{3}}=50 \mathrm{~nm}$ \\
\hline
\end{tabular}

Interesting to notice that the surface resistance plateau for $q_{0}>200 \mathrm{~nm}$ of curve $b$ corresponds to the surface resistance value obtained for a single pinning point at $2 \mathrm{~nm}$ (green up triangle $-\Delta-$ on curve $a$ ). This means that if $q_{0}$ is too large the second pinning point does not perturb the vortex behavior.

Adding a second fixed pinning point at $q_{0_{2}}=20 \mathrm{~nm}$ (red dots) and defining the abscissa as the position of the third pinning point, we obtain curve $c$ (Figure 4.5a). Also this time the surface resistance value is lowered for all the $q_{0}$ values.

Also in this case, the surface resistance is constant above a certain threshold $\left(q_{0}>100 \mathrm{~nm}\right)$, and approaches the values it would have if only two pinning points were present (green diamond- - - on curve $b$ ).

Instead, Figure $4.5 \mathrm{~b}$ shows the surface resistance as a function of the mean free path considering one (curve 1), two (curve 2) and three (curve 3) pinning points per vortex line, positioned at $2 \mathrm{~nm}, 2$ and $20 \mathrm{~nm}$ and 2, 20 and $50 \mathrm{~nm}$ respectively.

Since the pinning point number and position play a role only in the low mean free path region, as expected, no variation of the mean free path dependence are showed in the flux flow regime range. Noticeable variations of the trend are instead observable in the peak position and in the low mean free path region.

In case of multiple pinning, the peak changes position and moves towards larger mean free path values. Such phenomenon is symptom of an overall larger 

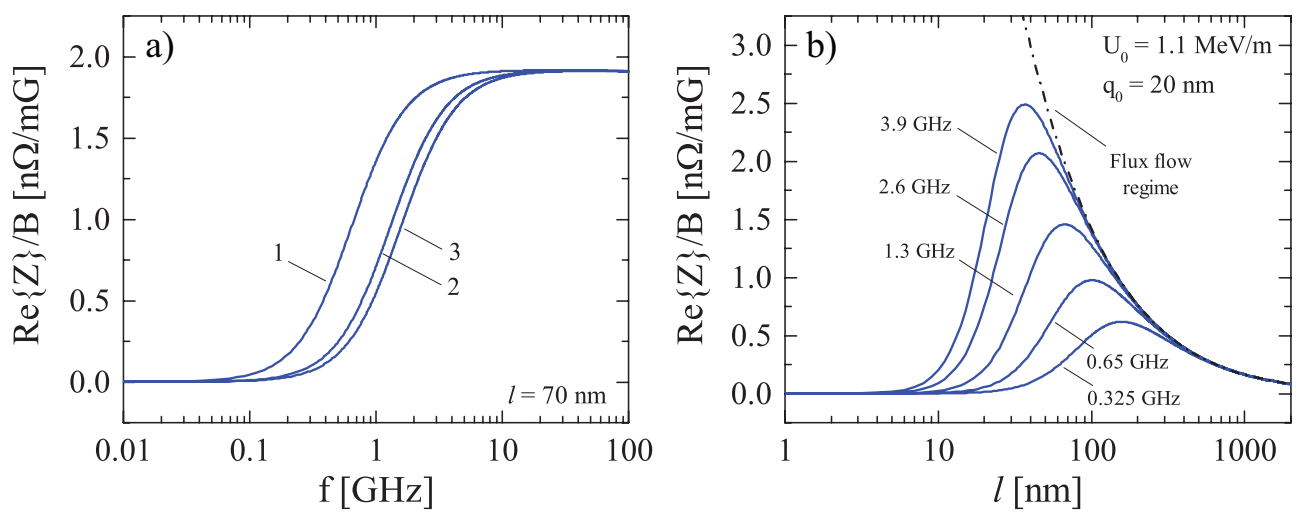

Figure 4.6. Frequency dependence of the surface resistance. in (a) the real part of the surface impedance is plotted as a function of the frequency, where curves 1,2 and 3 corresponds to one, two and three pinning points. In (b) the surface resistance calculated for a single pinning point at $q_{0}=2 \mathrm{~nm}$ is plotted as a function of the mean free path for increasing frequency values.

pinning force, i.e. the flux flow regime starts to take over at larger values of mean free path. Such larger pinning force acts also on the peak height, which is lowered in case of multiple pinning. Therefore a larger number of pinning points assures lower resistance. The green points with same shape in Figure 4.5a and b (up triangle $-\Delta$, diamond $-\diamond$ and down triangle $-\nabla)$ refers to surface resistance values calculated with the same parameters as reported in Table 4.2 .

4.3.3 Frequency Dependence. Up to this point we have considered always 1.3 GHz as constant frequency. Such frequency was selected inasmuch it is the most commonly used in SRF basic research and lots of experimental data are available. On the other hand though, the frequency dependence of the vortex surface impedance is of extreme importance.

In Figure 4.6a is reported the dependence of the real part of the vortices surface impedance as a function of the exciting frequency $f$. As shown by J. I. Gittleman and B. Rosenblum [77], the vortices surface resistance as a function of $f$ approaches two different limits: i) the high frequency regime where the flux flow dominates, and ii) the low frequency regime where the pinning dominates. 


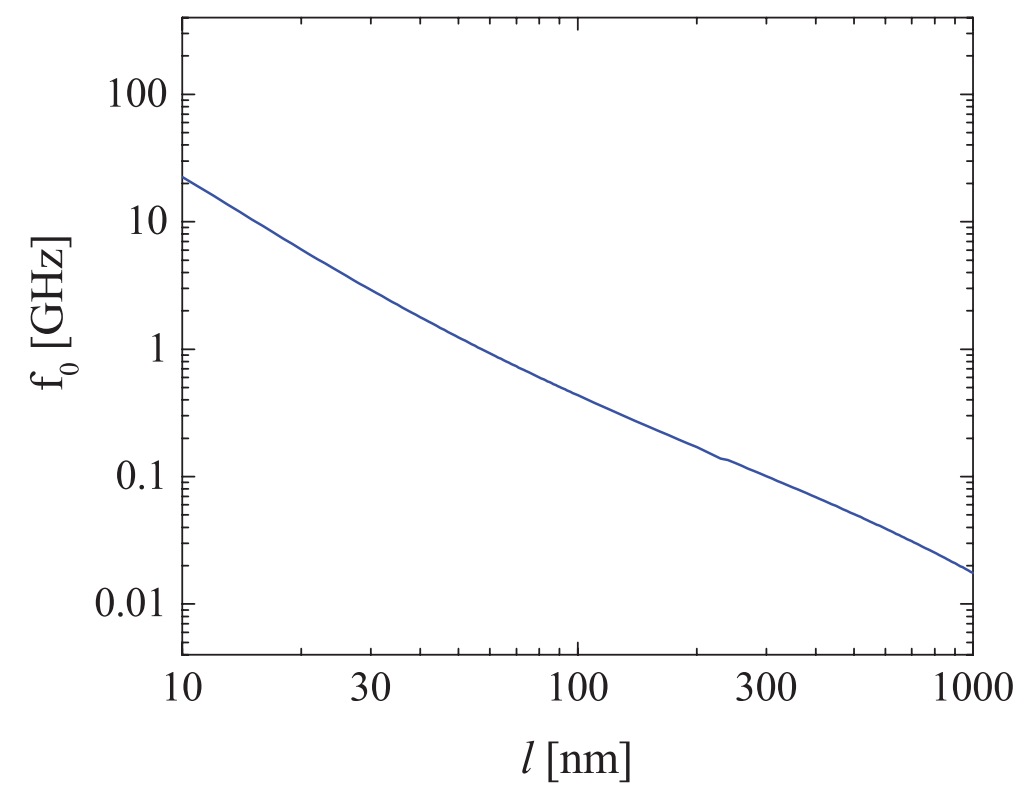

Figure 4.7. Depinning frequency as a function of the mean free path.

These two regimes are separated by the so-called depinning frequency $f_{0}$, which corresponds to the frequency where the pinning force is equal to the viscous drag force or, in other words, to "the frequency where the absorption reaches half of its ideal dc value" [77].

In the flux flow regime (above $f_{0}$ ), the pinning force is negligible and the dissipation is governed by the oscillation of the vortex within the pinning potential. Below $f_{0}$ the viscous drag force can be neglected, so the dissipation decreases since the vortex resistivity starts to assume a pure imaginary form (see Section 4.2.2).

When the frequency is increased the peak becomes taller and its position shifted to lower mean free path values. This happens because in the intermediate pinning regime (described by Eq. 4.17) the real part of the resistivity increases with the frequency squared, therefore for the same value of $l$ the surface resistance is larger for higher frequency values.

Such dependence on $f^{2}$ implies also that for high frequencies the perfect re- 
active vortex response is sustained up to lower values of mean free path. On the contrary, the lower the frequency the higher the mean free path values reachable in the condition of pure reactive response. In other words, for lower frequencies the onset of the flux flow regime occurs at higher values of mean free path, i.e. the peak moves towards larger $l$.

The simulations plotted in Figure 4.6a shows that the frequency dependence is function also of the number of pinning points (and their position). In particular, curve 1 corresponds to a single pinning point, curve 2 to two pinning points and curve 3 to three pinning points.

The number of pinning points modifies also the depinning frequency. The curves in Figure 4.6a are indeed shifted as a function of the number of pinning points. The depinning frequency - calculated as the frequency value at which the surface resistance assumes half of its dc value - is equal to about $0.64 \mathrm{GHz}, 1.29 \mathrm{GHz}$ and $1.57 \mathrm{GHz}$ for one, two and three pinning points respectively.

Experimental data obtained for $\mathrm{Nb}$ thin films [88] shows that the depinning frequency decreases as the thickness of the film increases, with $f_{0} \leq 1 \mathrm{GHz}$ for thicker films $(\sim 160 \mathrm{~nm})$, in agreement with our simulated $f_{0}$.

Figure 4.7 shows the depinning frequency dependence on the mean free path. The simulation was done considering $U_{0}=1.1 \mathrm{MeV} / \mathrm{m}$ and a single pinning point at $q_{0}=20 \mathrm{~nm}$. As shown, $f_{0}$ decreases with $l$, implying that the smaller the pinning force, the smaller the depinning frequency. As discussed before, by varying the mean free path is then possible to tune the depinning frequency, and therefore to define the vortices response regime: pinning regime for $f<f_{0}$ and flux flow regime for $f>f_{0}$.

\subsection{Model vs Experimental Data}

Several TESLA type [15] SRF niobium cavities were rf tested at the Fermi 


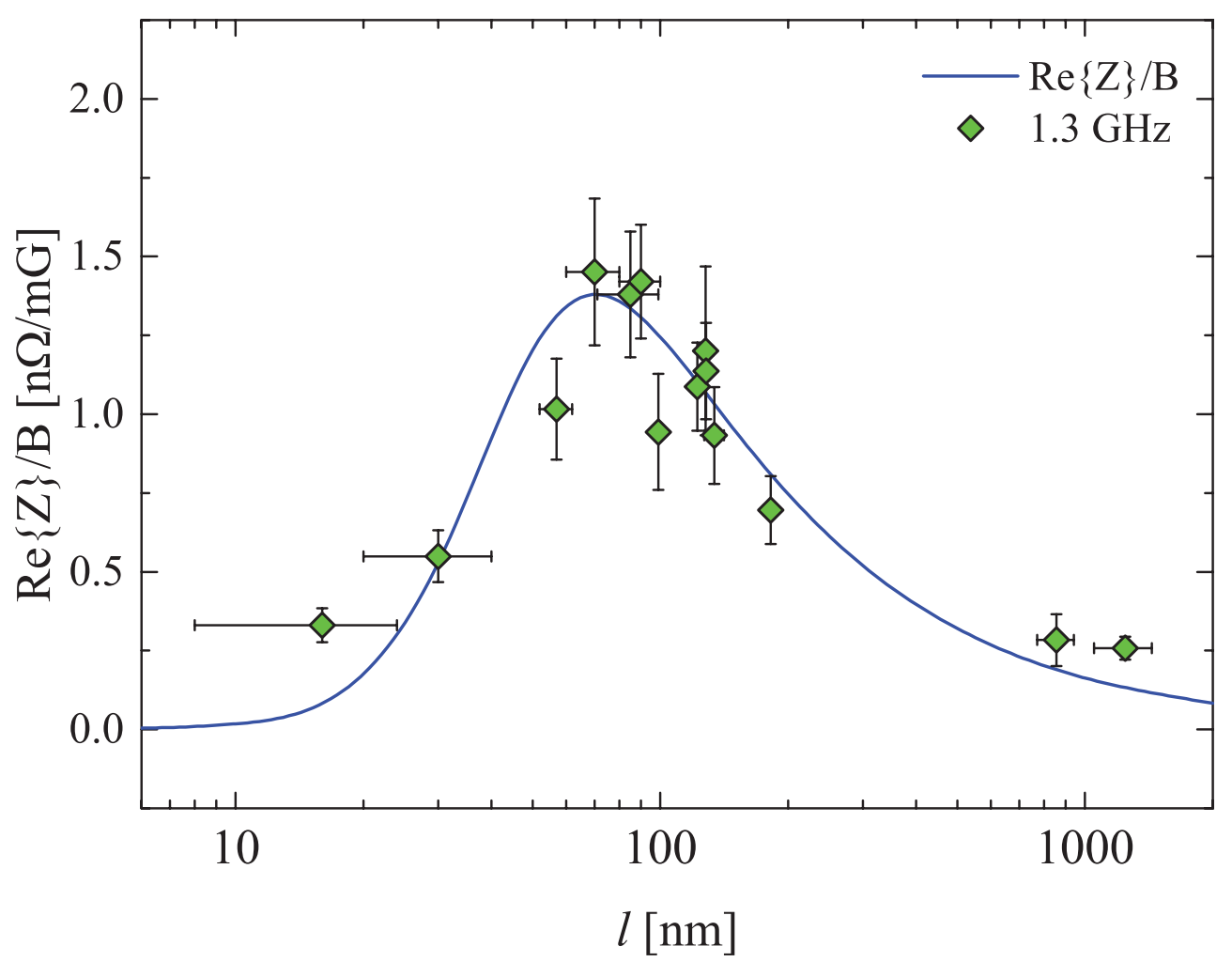

Figure 4.8. Extrapolated zero field data [10] and real part of the trapped flux surface impedance simulation.

National Accelerator Laboratory's the vertical test facility. The experimental procedures to measure the vortex surface resistance and the mean free path, along with the cavities preparation are reported in $[10,75,76]$.

The experimental data acquired [10] shows a linear dependence of the flux resistance as a function of the applied rf field expressed in $\mathrm{MV} / \mathrm{m}$. Since the model here developed is a zero field approximation (valid for rf fields tending to zero), the surface resistance dependence on the accelerating field reported in [10] was fitted linearly in order to extrapolate the surface resistance at zero field (the intercept).

In Figure 4.8, the experimental data extrapolated at zero accelerating field is plotted along with the real part of the surface impedance of Eq. 4.24. The solid curve plotted is the simulation of the vortex surface resistance assuming a single pinning 
point, with pinning point distribution centroids at $q_{0}=20 \mathrm{~nm}$ and $U_{0}=1.4 \mathrm{MeV} / \mathrm{m}$, and standard deviation $\sigma_{q_{0}}=30 \mathrm{~nm}$ and $\sigma_{U_{0}}=0.08 \mathrm{MeV} / \mathrm{m}$. The data acquired presents a bell-shaped trend in agreement with the model prediction, with a maximum around $70 \mathrm{~nm}$, and surface resistance decreasing for large and small mean free path values. In Appendix B the numerical code to calculate the surface resistance is reported.

The $U_{0}$ values used to obtain a satisfactory description of the data are consistent with the experimental data for niobium obtained by L. H. Allen and J. H. Claassen [89] and G. S. Park, et al. [90]. In the mean free path range $2-2000 \mathrm{~nm}$, the pinning potential strength returns values of maximum force per meter (the force per meter was assumed as equal to the maximum of $-\partial U_{p}(x) / \partial x$ as a function of $x$ calculated at $z=q_{0}$ ) equal to $8 \times 10^{-5}-4 \times 10^{-6} \mathrm{~N} / \mathrm{m}$. Such values are in perfect agreement with [89] $\left(10^{-5}-10^{-6} \mathrm{~N} / \mathrm{m}\right)$ and slightly underestimated with respect to $[90]\left(\sim 10^{-4} \mathrm{~N} / \mathrm{m}\right)$.

Because of the peculiar shape of TESLA cavities [15], the current density and the trapped flux directions can have intersection angle lower than ninety degrees, therefore $\sin ^{2} \theta$ (in Eq. 4.13) cannot be approximated to 1. It was indeed observed in several works $[91,92,93]$ that the vortex-related resistance varies consistently with the Lorentz force dependence on the angle between the directions of the currents and the trapped flux. In order to get reasonable resistance values, an average angle of about $18^{\circ}$ was considered, and $\sin ^{2} \theta$ was assumed equal to 0.1 .

The simulated curve describes the experimental data satisfactorily even by considering a single pinning point per flux line. The biggest scattering is observed in the peak area where the pinning force plays the central role in the vortex dissipation description. 
All the cavities measured are prepared with different procedures in order to ensure a good variability of mean free paths. It is therefore reasonable to assume that pinning potential depth and pinning site distributions $\left(\Gamma\left(q_{0_{i}}\right)\right.$ and $\left.\Lambda\left(U_{0_{i}}\right)\right)$, as well as the number of pinning points might be different from cavity to cavity. Such aleatory difference between the various cavities studied may explain the data scattering, as well as the discrepancies with the theoretical model. It is indeed unlikely that one single simulated curve can describe exhaustively the data all in once.

The presented model can in principle explain why between one and two orders of magnitude lower values of vortex-related surface resistance are observed for $\mathrm{Nb}$ on $\mathrm{Cu}$ SRF cavities [94], compared to our experimental results. Because of niobium thin films are more defective (e.g. porosity, columnar growth, etc.), the pinning force might be substantially larger than in bulk $\mathrm{Nb}\left(U_{0}\right.$ and the number of pinning points might be larger). In such scenario, the perfect reactive response of vortices might survive up to larger mean free path values and the peak shifted towards larger $l$, that have not been surveyed yet for the thin film technology.

Recently, $\mathrm{Nb}_{3} \mathrm{Sn}$ vortex surface impedance data forof SRF cavities became available [95], showing comparable vortex surface resistance with respect to dirty niobium. In principle, one would expect higher vortex surface resistance in the flux flow regime because of the higher resistivity of $\mathrm{Nb}_{3} \mathrm{Sn}$ in the normal-conducting state. On the other hand though, $\mathrm{Nb}_{3} \mathrm{Sn}$ was shown to posses high pinning force at the grain boundaries $[96,97,98]$, which would be in agreement with an overall suppressed vortex surface resistance, as experimentally observed.

\subsection{Summary}

In this chapter, the explicit description of the vortex-related surface impedance as a function of the mean free path at $\mathrm{rf}$ and microwave frequencies is proposed. The 
problem was approached by assuming a bi-dimensional pinning potential dependent on the electrons mean free path and by solving the single-vortex motion equation. Differently than previous works, an explicit dependence of the vortices surface resistance on the mean free path was found, and the dependence of the surface resistance as a function of the number, disposition and strength of the pinning points in the material was studied.

The experimental data observed for different SRF niobium cavities at $1.3 \mathrm{GHz}$ can be explained exhaustively by the interplay of the limiting responses of the surface resistance for low and large values of mean free path: the pinning and the flux flow regime respectively. Because of the different thermal history of every cavity, the experimental data shows some scattering: the pinning position and strength distributions may indeed be different. This means that the model here presented does provide us an average description of the experimental data.

The bell-shape trend experimentally observed is generated by the variation of the vortices response from totally resistive at large values of mean free path, to totally reactive for small $l$. In the pinning regime (small $l$ ) the pinning force is governing the vortices response since for small $l$ the viscous drag force is negligible. Hence, in absence of dissipative mechanisms, the response is totally reactive and the surface resistance tends to zero. As the mean free path increases, the viscous drag force increases driven by the decreasing of the normal-state resistivity, meanwhile the pinning force becomes negligible because of a larger coherence length. Consequently, the surface resistance follows increasing.

Above a certain mean-free-path value threshold-defined by frequency, position, number and strength of pinning points - the flux-flow regime takes over and the surface resistance assumes its maximum value. For larger values of $l$, the surface resistance decreases driven by the increment of the drag force - the surface resistance 
in the flux-flow regime is inversely proportional to the viscous drag coefficient.

Such behavior of the surface resistance with the mean free path is extremely important since introduces the mean free path as another variable able to tune the dissipation regime other than the frequency. The model predicts that position, strength and number of pinning sites can modify substantially the vortex surface resistance and the depinning frequency, shifting and affecting the maximum of surface resistance as a function of the mean free path.

Pinning points near the rf surface affects the vortices response lowering the surface resistance. On the contrary, when the pinning site is far enough from the rf surface its presence does not perturb anymore the vortex oscillation and the resistance approaches to its constant and maximum value.

The frequency dependence shows the typical trend expected, but observing the surface resistance as a function of the mean free path the model predicts that higher frequencies shift the peak to small $l$ values and increase its maximum, because of the different balance of forces - larger frequencies allows the real part of the resistivity to grow faster when moving from low to large values of mean free path, enhancing the peak maximum and shifting it to lower mean free path values. 


\section{CHAPTER 5 \\ ACCELERATING GRADIENT LIMITATIONS}

\subsection{Chapter Overview}

When the cavity is operating in the accelerator, or simply being tested in a vertical test facility, the accelerating gradient cannot be increased indefinitely. Because of the of the superconducting nature of niobium, when the maximum bearable field is reached in the resonator the sudden increase of the surface resistance consumes all the rf energy stored in the cavity causing the so-called quench.

The physical understanding of the quench nature is then of critical importance in order to develop new technologies to enable superconducting particles accelerators to operate at higher energies. The scope of this chapter is indeed to advance the understanding of the fundamentals limitations of cavities in terms of accelerating field.

The accelerating gradient limitation can be categorized in two different classes: i) the fundamental limitation introduced by the overcoming of the field of first penetration - field at which vortexes can penetrate the superconductor, or ii) non-fundamental, and therefore introduced by the presence of limiting factors such as defects at the rf surface or associated to phenomena such as multipacting or field emission. The nonfundamental quench triggering mechanisms in SRF cavities are nowadays ascertained

and well described in many references $[13,99]$, hence only a briefly explanation of the three more general quench mechanisms is here presented.

The central topic of this chapter is instead addressed to the understanding of the fundamental limitations of cavities as a function of their material properties. Indeed, as discussed in Section 3.3, different thermal treatments not only affect the quality factor, bu also the accelerating gradient. 
Since the fundamental limitation of the accelerating gradient is the penetration of magnetic vortices in the material, the fully description of the vortex stability in the superconductor as a function of the rf magnetic field inside the cavity is needed. At $2 \mathrm{~K}$ and low field values $\left(B<B_{c 1}\right)$, niobium is in the Meissner state, therefore vortices should not penetrate until the lower critical field value is matched. Anyway, the Meissner state might be sustained in a meta-stable condition up to the superheating field $B_{s h}$, where $B_{s h}>B_{c 1}$. The resonator should then be able to reach higher fields than what is described by the pure equilibrium thermodynamics point of view.

By means of the dimensionless Ginzburg-Landau equations defined in Section 2.2, the theoretical values of lower critical field and superheating field as a function of the Ginzburg-Landau parameter $\kappa$ are calculated numerically. Such theoretical trends are then compared with some available quench field data for SRF cavities. The direct comparison of theory and experimental data suggests that the so-called $120{ }^{\circ} \mathrm{C}$ baking is the only standard treatment capable to systematically push cavities above the lower critical field in the meta-stable Meissner state.

\subsection{Description of the Quench Phenomenon}

A typical non-fundamental quench event is initiated by a small area of the cavity surface becoming normal conducting either due to heating up above the critical temperature $\left(T_{c}\right)$, or due to the local field of first penetration being exceeded. The sharp increase of the surface resistance in the normal zone can only be contained up to a certain dissipation level, above which a fast avalanche-like spreading of the normal zone that consumes all of the rf field in the cavity occurs.

When the totality of the electromagnetic energy stored in the resonator is dissipated in the quench region, the normal-conducting zone can turn again to superconductive cooled by the helium bath. At this point, the electromagnetic field is 
reestablished into the resonator since fed by the rf system. When the field level in the cavity reaches again the quench field, the cavity will quench again, and the whole process will be repeated. It is then possible to tune the rf system in such a way to quench the cavity repeatedly any time the field replenished in the resonator reaches the quench field value.

5.2.1 Thermal Breakdown. The thermal breakdown is a quench mechanism initiated by the presence of defects on the rf surface, that introduces losses significantly higher than the surrounding superconductor of which the resonator is made of $[13$, $99,100]$.

In dc case, the current in the superconductor flows around the defect since following the path of lower resistance. In the rf case though, the currents that sustain the field in the cavity flow on all the rf surface, meaning that also the defect contributes the total power loss. Since the dissipation is enhanced at the defect location because of its larger resistance, in such area the quench event can be initiated by the local overheating above the transition temperature. The normal-conducting zone generated is then be able to grow by dissipating the energy stored in the cavity till the cavity quenches.

Defects usually recognized as "quench defects" can either be superconducting or normal-conducting, the only common feature is their increased surface resistance. Such kind of quench, so-called thermal breakdown, is usually diagnosed by meaning of the temperature mapping system (see Subsection 3.2.5). In such occasions when the resonator quenches because of thermal breakdown, an increment of the temperature at the quench location as a function of the accelerating field - preheating - is usually observed during the power rise.

5.2.2 Magnetic Field Enhancement. The magnetic field enhancement quench 
mechanism assumes the presence of a superconducting defect, that enhances locally the magnetic field and promotes the transition to the normal-conducting state to take place at the quench spot $[99,101,102]$.

At the cavity operational temperature (about $2 \mathrm{~K}$ ), the behavior of niobium is perfectly diamagnetic because of the Meissener effect, therefore the magnetic field is excluded from the material. Since the rf magnetic field is always parallel to the surface, high aspect ratio imperfections of the superconducting rf surface can in principle enhance the field locally because of the demagnetization effect (see Section 2.3). In such scenario, even if the field level in the cavity is far from the theoretical limit imposed by the field of first penetration, the cavity may quench anyway since locally the field is actually larger than the field of first penetration - no heating above $T_{c}$ needed.

Defects that in principle can generate such effect are: pits, bumps and steep grain boundaries. Also such quench mechanism is usually diagnose by meaning of the T-map system. When no preheating is observed at the quench location before the quench event, the magnetic field enhancement is the only possible quench mechanism, since it is the only triggering phenomenon that does not require a temperature raising to quench the resonator.

5.2.3 Field Emission- and Multipacting-driven quench. Field emission and multipacting are two phenomena involving acceleration of electrons in the cavity that absorb rf power [13].

Field emission consists in the emission of electrons from the rf surface driven by the high value of electric field at the cavity rf surface (see Subsection 3.1.3). Field emission is generally observed at the cavity irises especially when sharp particles are present. Field emitted electrons are accelerated by the rf field in the cavity and 
a large part of them end up colliding with the cavity surface generating X-rays by Bremsstrahlung. The interaction between electrons and niobium produces also heat that increases the local temperature. If the temperature rises high enough the cavity quenches.

In case of multipacting, the situation is slightly different. Multipacting is a resonant mechanism in which a large number of electrons build up spontaneously absorbing rf power [13]. The trajectories of such electrons are resonant, meaning that the electrons are initially accelerated and then bent by the rf magnetic field. In such a way, the electrons in the resonance condition collide with the rf surface in the same position (or nearby it), from where they were emitted. If the secondary electrons yield coefficient is larger than 1, then a cascade phenomenon takes place. Both X-rays and heat are produced, and for the same reason of field emission this phenomenon may cause the quench of the resonator.

5.2.4 The Ultimate Gradient Limitation. As already introduced, the fundamental limitation to the highest reachable accelerating field in the cavity is the field of first penetration, at which magnetic flux vortexes are allowed to penetrate the superconductor. Neglecting the vortex pinning, the field of first penetration ranges between two limits: i) the lower critical field $H_{c 1}$ as lower limit, and ii) the superheating field $H_{s h}$ as upper limit. In particular:

i the lower critical field $H_{c 1}$ corresponds to the field level at which non-interacting magnetic flux vortexes are stable in the bulk of the superconductor, i.e. the Gibbs free energy is equal to zero;

ii the superheating field $H_{s h}$ is defined as the highest field at which magnetic flux may penetrate from the surface, i.e. the highest applied field for which the Ginzburg-Landau free energy still possess a local minimum as a function of the 
order parameter.

In order to investigate such ultimate field limitation, the full description of both the lower critical field and the superheating field for arbitrary values of GinzburgLandau parameter $\kappa$ is needed. Analytic formulae to describe $H_{c 1}$ and $H_{s h}$ are reported in literature $[25,33]$ in the approximation $\kappa \gg 1$. In our case, experimentally calculated values of $\kappa[10]$ for the SRF cavities studied show $1 \lesssim \kappa \lesssim 10$, meaning that such analytic approximations are not always valid of our purposes. In the following sections numerical values of $H_{c 1}$ and $H_{s h}$ for arbitrary $\kappa$ are calculated starting directly from the Ginzburg-Landau equations, in order to compare them with the experimental data acquired.

\subsection{Lower Critical Field Numerical Calculation}

The lower critical field of a type-II superconductor is defined as the field at which a single magnetic flux quanta (vortex), not interacting with other vortices and far from any surface, is stable in the bulk of the superconductor.

The simplest method to describe such situation is then to analyze the problem by defining the Gibbs free energy density when vortices are not interacting $\left(H \ll H_{c 2}\right)$. The Gibbs free energy density in presence of magnetic field is simply written as $G=F+B M$ where $F$ is the Helmholtz free energy density, $B$ is the magnetic induction and $M$ the magnetization. In the Meissner state- when the applied field $H$ is below $H_{c 1}$ - the magnetic induction in the superconductor is zero, which means that:

$$
B=\mu_{0}(H+M)=0 \quad \rightarrow \quad M=-H
$$

and the Gibbs free energy density then has form:

$$
G=F-B H
$$


For low vortices areal density $n$ (just above $H_{c 1}$ ), the Helmholtz free energy density is equal to $n$ times the energy of a single vortex per unit of length $E$, and $n=B / \phi_{0}\left(\right.$ with $\phi_{0}=2.07 \cdot 10^{-15} \mathrm{~Wb}$, the flux quantum $)$. We can therefore rewrite $G$ as:

$$
G=B\left(\frac{E}{\phi_{0}}-H\right)
$$

The lower critical field corresponds to the field $H$ for which the Gibbs free energy density is equal to zero. Equating Eq. 5.3 to zero and rearranging the terms, we get the definition of lower critical field:

$$
H_{c 1}=\frac{E}{\phi_{0}}
$$

We can now take advantage of the notation used in [103] to turn such equation dimensionless:

$$
h_{c 1}=\frac{\epsilon \kappa}{4 \pi}
$$

where $h_{c 1}=H_{c 1} /\left(\sqrt{2} H_{c}\right)$ and $\epsilon$ is the dimensionless energy per unit of length of a vortex in the superconductor, defined as in [26]:

$$
\epsilon=\int_{0}^{\infty}\left[h^{2}(r)+\frac{1}{2}\left(1-f^{4}(r)\right)\right] 2 \pi r d r
$$

with $h(r)=H(r) /\left(\sqrt{2} H_{c}\right)$ and $f(r)=\Psi(r) / \Psi_{0}$ being the dimensionless radial profile of the local magnetic field and the order parameter of a vortex respectively.

In order to get the values of both $h(r)$ and $f(r)$ for a vortex in a superconductor with arbitrary $\kappa$, we must solve numerically the dimensionless Ginzburg-Landau equations (Eq. 2.13). Before doing that, the problem must be prepared analytically by expressing such equations in cylindrical symmetry.

Let us substitute the complex order parameter, constituted of modulus $f$ and phase $\varphi$, instead of the general order parameter $f$ till now assumed:

$$
f \rightarrow f e^{i \varphi}
$$


and vector potential in dimensionless notation, a, perpendicular to the radius $r$. The third term of the first equation in Eq. 2.13 becomes:

$$
\begin{aligned}
\left(\mathbf{a}+\frac{i \nabla}{\kappa}\right)^{2} f e^{i \varphi}= & \left(a^{2}-\frac{\nabla^{2}}{\kappa^{2}}+\frac{i 2 \mathbf{a} \cdot \nabla}{\kappa}\right)^{2} f e^{i \varphi} \\
= & -\frac{1}{\kappa^{2}} e^{i \varphi}\left\{\left(f^{\prime \prime}+\frac{1}{\kappa} f^{\prime}\right)+\frac{1}{r^{2}} f\left[-\left(\frac{\partial \varphi}{\partial \theta}\right)^{2}+i \frac{\partial^{2} \varphi}{\partial \theta}\right]\right\}+ \\
& +e^{i \varphi}\left[\frac{i 2 a}{\kappa} f^{\prime}(\hat{\theta} \cdot \hat{r})-\frac{2 a}{\kappa r} f\left(\frac{\partial \varphi}{\partial \theta}\right)\right]+a^{2} f e^{i \varphi}
\end{aligned}
$$

where the prime identifies the derivative with respect the normalized radius $r$. As assumed by A. A. Abrikosov [26], the cylindrical symmetry of the system imposes that $\varphi \equiv \theta$ meaning that $\partial \varphi / \partial \theta=1$. Furthermore, since $\mathbf{a}$ is orthogonal to $\mathbf{r}$ the scalar product $(\hat{\theta} \cdot \hat{r})=0$. We can therefore simplify the equation, and after some algebra we obtain:

$$
f^{\prime \prime}+\frac{1}{r} f^{\prime}-\kappa^{2} f\left[f^{2}-1+\left(a-\frac{1}{\kappa r}\right)^{2}\right]=0,
$$

that corresponds to the first dimensionless Ginzburg-Landau equation in cylindrical coordinates.

We should now assume the second equation in Eq. 2.13. In this case, using the same assumptions, we can rewrite the first and second terms as:

$$
\begin{aligned}
& \nabla \times(\nabla \times \mathbf{a})=-\mathbf{a}^{\prime \prime}-\frac{1}{r} \mathbf{a}^{\prime}+\frac{1}{r^{2}} \mathbf{a} \\
& \frac{i}{2 \kappa}\left[f e^{-i \varphi} \nabla\left(f e^{i \varphi}\right)-f e^{i \varphi} \nabla\left(f e^{-i \varphi}\right)\right]=-\frac{1}{\kappa r} f^{2} \hat{\theta} .
\end{aligned}
$$

Hence, the second Ginzburg-Landau equation that describes a vortex line in cylindrical coordinates is:

$$
\mathbf{a}^{\prime \prime}+\frac{1}{r} \mathbf{a}^{\prime}-\frac{1}{r^{2}} \mathbf{a}-f^{2}\left(\mathbf{a}-\frac{1}{\kappa r} \hat{\theta}\right)=0 .
$$

Eqs. 5.9 and 5.11 describes the single vortex in the superconductor for arbitrary values of Ginzburg-Landau parameter $\kappa$. 
In order to solve such equations numerically, we must assume a certain domain extension where these equations are defined, as well as the appropriate boundary conditions. The domain chosen has lower limit equal to $r_{0}=10^{-5}$ and higher limit $R$ to be determined self-consistently. In particular, $r_{0}$ was chosen bigger than zero since both the two equation, Eqs. 5.9 and 5.11, present a singularity at $r=0$. Since numerical differential equations solvers cannot handle such singularities, the lower limit of the domain $\left(r_{0}\right)$ was set equal to a small number, which was assured of not affecting the final result of the calculation. The boundary conditions assumed are:

$$
\begin{array}{ll}
f\left(r_{0}\right)=0, & f(R)=1 \\
a\left(r_{0}\right)=0 & , \quad a(R)=\frac{1}{\kappa R}
\end{array}
$$

where $a(r)=1 /(\kappa r)$ is the asymptotic form of the vector potential at infinity. For $r \rightarrow \infty, a=0$.

The boundary value problem (BVP) defined by Eqs. 5.9, 5.11 and 5.12 was then solved numerically with a Wolfram Mathematica code by means of a self-consistent shooting method (see Appendix $\mathrm{C}$ for shooting method description), where the solution domain $R$ was increased iteration after iteration till the correct solution was achieved. In Figure 5.1, the numerical code is represented in a flow chart, while in Appendix D the Wolfram Mathematica code is reported.

The code inputs are the BVP defined by Eqs. 5.9, 5.11 and 5.12, plus the initial conditions of the initial value problem (IVP):

$$
\begin{array}{ll}
f\left(r_{0}\right)=0 \quad, \quad f^{\prime}\left(r_{0}\right)=f 1 \\
a\left(r_{0}\right)=0 \quad, \quad a^{\prime}\left(r_{0}\right)=a 1
\end{array}
$$

where $f 1$ and $a 1$ are calculated self-consistently from the numeric code.

The code is written as two concatenated loops. The first one iterates on all the $\kappa$ values from the minimum value $\kappa_{\min }$ to the maximum value $\kappa_{\max }$, with a step 


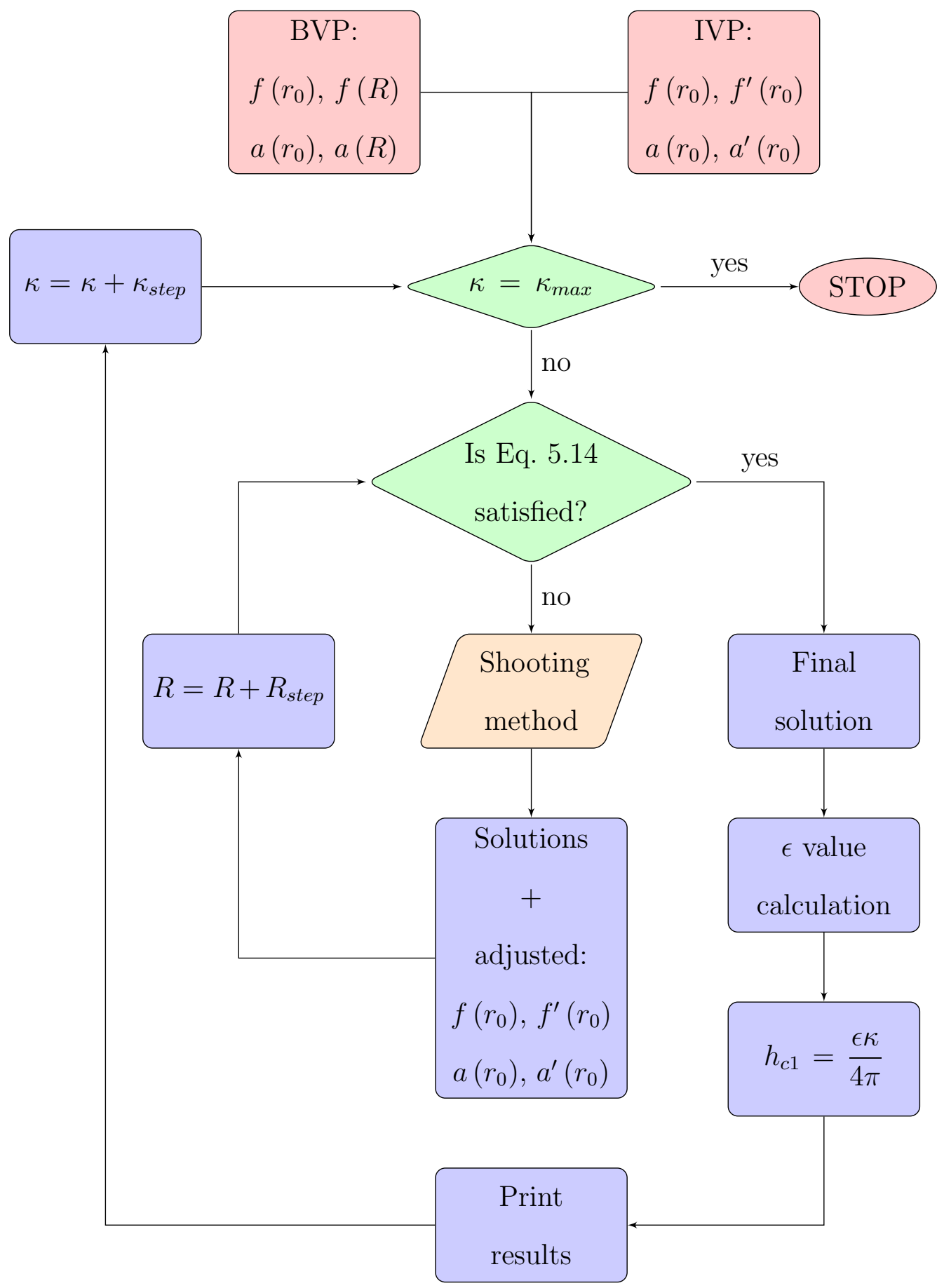

Figure 5.1. Flow chart representing the self-consistent numerical code to calculate $h_{c 1}$. 

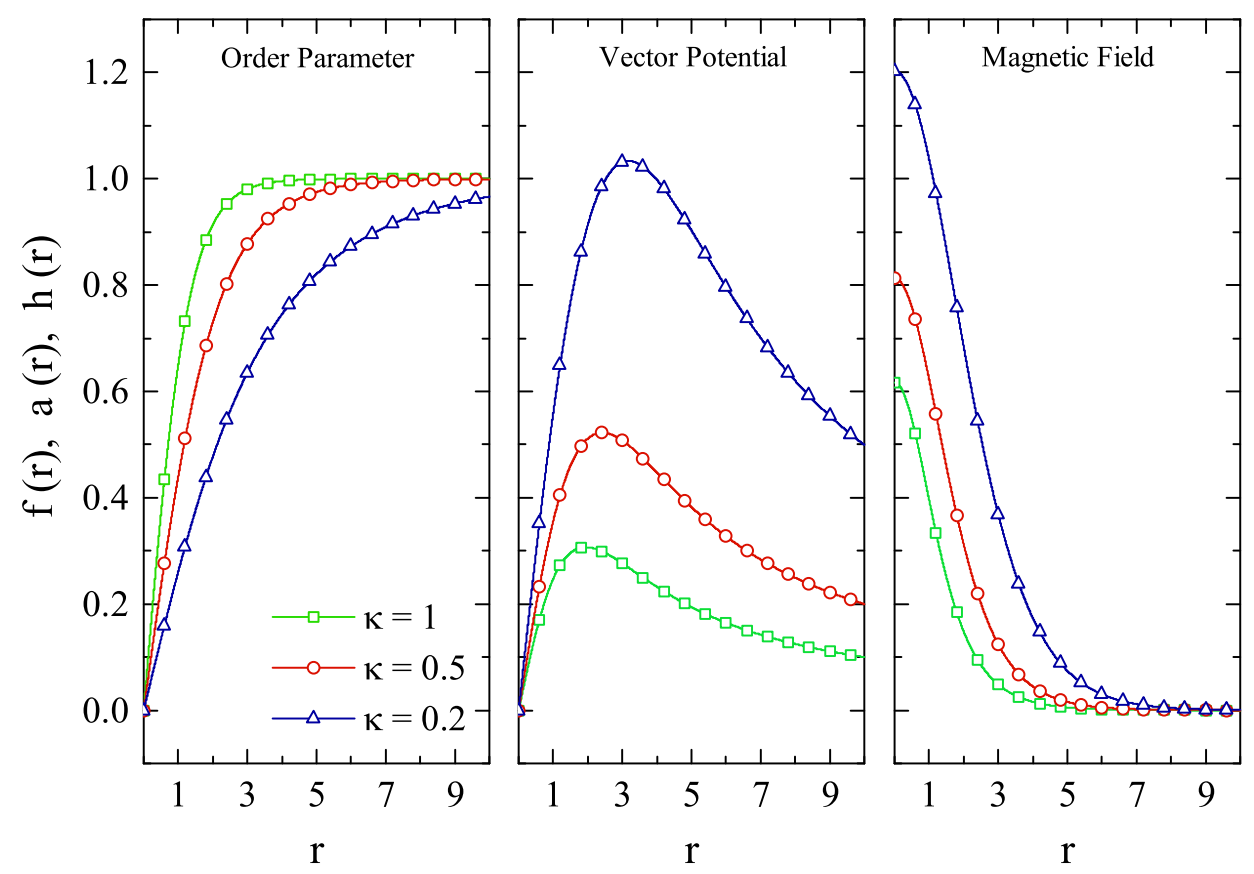

Figure 5.2. Numerical solution of the Ginzburg-Landau equations in cylindrical symmetry for different values of $\kappa$.

equal to $\kappa_{\text {step }}$. The second loop is needed to increase iteratively the dimension of the solution domain $R$. Such second cycle is extremely important: given the initial values it calculates the solutions and returns the adjusted initial values of $f 1$ and $a 1$ (Eq. 5.13) at every cycle. In such a way, at every cycle the solution domain $R$ is increased till the exit condition is matched. The exit condition used is the following:

$$
\left\{\begin{array}{c}
{\left[R>5 \wedge\left(f(R)>1.001 \vee f^{\prime}(R)<0\right)\right]} \\
\vee \\
{\left[R>5 \wedge\left(a^{\prime}(R) \geq 0 \vee a^{\prime}(R) \leq-\frac{1}{\kappa R^{2}}\right)\right]} \\
\vee \\
R=20
\end{array}\right.
$$

The first condition in Eq. 5.14 controls the shape of the solution every iteration. The order parameter shape must be flat $\left(f^{\prime}(R)=0\right)$ at the domain boundary and with 
value equal to one $(f(R)=1)$. At the same time, also the vector potential should be monitored. Even if at the boundary its slope must be $-1 /\left(\kappa R^{2}\right)$, its shape may vary a bit during the various iterations. In order to let the solution to converge correctly, such slope must fall inside the range $-1 /\left(\kappa R^{2}\right) \leq a \leq 0$. These boundary conditions are matched only asymptotically, therefore in both cases the exit condition imposes also that $R$ must be larger than 5. Such precaution assures that the domain is large enough that the solution already tends towards the asymptotic boundary conditions imposed.

The second condition is imposed on the maximum extension of the domain. Such maximum value was chosen inasmuch it minimizes the computation time without affecting the solution accuracy. As just said, the boundary conditions should be matched at infinite, therefore the larger the solution domain, the more accurate the solution.

Once the exit condition is matched, the code calculates the single non-interacting vortex energy $\epsilon$ from Eq. 5.6 by means of the numerical functions $f(r)$ and $h(r)$. The normalized lower critical field is then obtained from Eq. 5.5 for a particular $\kappa$ value. The entire program is then reiterated increasing $\kappa$ till the $\kappa_{\max }$ limit is matched.

In Figure 5.2, three numerical simulations of order parameter, vector potential and local magnetic field of an isolated vortex as a function of the normalized radius $r$ $(=\operatorname{radius} / \lambda)$ are shown. The coordinate zero corresponds to the vortex center, which as described by A. A. Abrikosov [26] is the point at which both order parameter and vector potential assume zero value. On the opposite, the magnetic field has maximum value at the vortex center. Far from the vortex center the order parameter tends to 1, while the vector potential and magnetic field tend to zero, the first one assuming an asymptotic form $a \rightarrow 1 /(\kappa r)$. 


\subsection{Superheating Field Numerical Calculation}

The superheating field of superconductors corresponds to the highest field for which the Ginzburg-Landau free energy still possess a local minimum as a function of the order parameter. In other words, $H_{s h}$ corresponds to the highest field at which the superconductive Meissner state can exist in a meta-stable condition above the lower critical field.

Since the Ginzburg-Landau equations defines the coupled functions order parameter and vector potential for which the free energy density is minimized, solving such equations for increasing values of applied magnetic field returns the value of the superheating field. In particular, the superheating field corresponds to the highest magnetic field for which we still find an acceptable solution of the Ginzburg-Landau equations.

The geometry of the problem is different than before (Section 5.3). We are now considering a semi-infinite superconductor for $x>0$, with surface at $x=0$, and applied magnetic field parallel to the surface along the $\hat{z}$ direction. The superconducting screening currents flow at the surface along the $\hat{y}$ direction. In such geometry the order parameter is a pure real number.

We can now rewrite the Ginzburg-Landau equations in order to match such geometry. Let us consider the first equation in Eq. 2.13. The third term becomes:

$$
\begin{aligned}
\left(\mathbf{a}+\frac{i \nabla}{\kappa}\right)^{2} f & =\left(a^{2}-\frac{\nabla^{2}}{\kappa^{2}}+\frac{i 2 \mathbf{a} \cdot \nabla}{\kappa}\right) f \\
& =a^{2} f-\frac{1}{\kappa^{2}} f^{\prime \prime}+\frac{i 2 a f^{\prime}}{\kappa}(\hat{y} \cdot \hat{x}),
\end{aligned}
$$

where the prime identifies the derivative with respect the $x$ coordinate. Since $\hat{y}$ is orthogonal to $\hat{x}$ the third term is zero, and the first Ginzburg-Landau equation takes form:

$$
\frac{1}{\kappa^{2}} f^{\prime \prime}-a^{2} f+f-f^{3}=0 .
$$


We should now consider the second Ginzburg-Landau equation in Eq. 2.13 and rewrite the first and second terms as:

$$
\begin{gathered}
\nabla \times(\nabla \times \mathbf{a})=-\mathbf{a}^{\prime \prime} \\
\frac{i}{2 \kappa}[f \nabla f-f \nabla f]=0 .
\end{gathered}
$$

Substituting back such definitions, we obtain the second Ginzburg-Landau equation in the mono-dimensional case:

$$
\mathbf{a}^{\prime \prime}-f^{2} \mathbf{a}=0 .
$$

Eq. 5.16 and 5.18 represent the Ginzburg-Landau equations describing the penetration of the magnetic field applied parallel to the surface of the superconductor in a one-dimensional case, as a function of the of the material's $\kappa$.

The numerical calculation was performed with a self-consistent Shooting Method (see Appendix C), assuming the following boundary conditions:

$$
\begin{array}{ll}
f^{\prime}(0)=0 & , \quad f(X)=1 \\
a^{\prime}(0)=h & , \quad a(X)=0
\end{array}
$$

where $h$ is the applied field parallel to the superconductor surface and $X$ the maximum extension of the solution domain. Since we are dealing with a numerical shooting method solver the following initial conditions are also provided:

$$
f(0)=f 1 \quad, \quad a(0)=a 1
$$

with $f 1$ and $a 1$ calculated self-consistently.

The numerical code is composed of three concatenated loops. The first one iterates on all the possible values of $\kappa$, from a minimum value $\kappa_{\min }$ to a maximum value $\kappa_{\max }$, with steps equal to $\kappa_{\text {step }}$. The second one increases the value of the applied field at the surface $h$ at every iteration, of an amount $h_{\text {step }}$, while the third one is implemented in order to increase the dimension of the solution domain $X$ by steps 
equal to $X_{\text {step }}$. Inside this last loop, there are three different exit conditions: the first one probes the accuracy of the solution obtained, the second one is needed to control the validity of the solution and the third one monitors if the numerical solver fails to return a solution. In Appendix D the numerical code is reported, while in Figure 5.3 the corresponded flow chart is showed.

The first exit condition is the following:

$$
\left[X>5 \wedge f(X)>1.001 \wedge f^{\prime}(X)<0\right] \vee X=8,
$$

and it guarantees that when the numerical solution starts to deviate substantially from the ideal one $\left(f(X)=1\right.$ and $\left.f^{\prime}(X)=0\right)$, the inner loop on the domain extension $X$ is stopped and the computation moved to the intermediate loop on the applied field $h$. At this point the field is increased and the inner loop on $X$ re-initialized.

Inside the inner loop, the second exit condition is represented by a test on the order parameter value at the surface. If $f(0)$ calculated by the numerical solver assumes values too low $(<0.0001)$ the solution calculated is assumed to be "normalconductive", i.e. when the order parameter is zero, in all or at the surface of the superconductor, the material is normal-conducting. When such condition is matched the inner and the middle loops are stopped and the logic flag normally set on False is turned on True. The code then prints the superheating field value $\left(h_{s h}\right)$ for that particular $\kappa$, which corresponds to the last value of $h$ minus the field step $h_{\text {step }}$. The value of $\kappa$ is then increased and the process repeated.

Important to point out that not always the Ginzburg-Landau equations can be solved for fields larger that $h_{s h}$ giving "normal-conductive" solutions. Often (usually depending on the $X_{\text {step }}$ and $h_{\text {step }}$ values implemented), the numerical solver can return a non-real solution when $h>h_{s h}$. The third exit condition takes care of stopping the inner and the middle loops when non-real solutions are returned, and turns the logic 


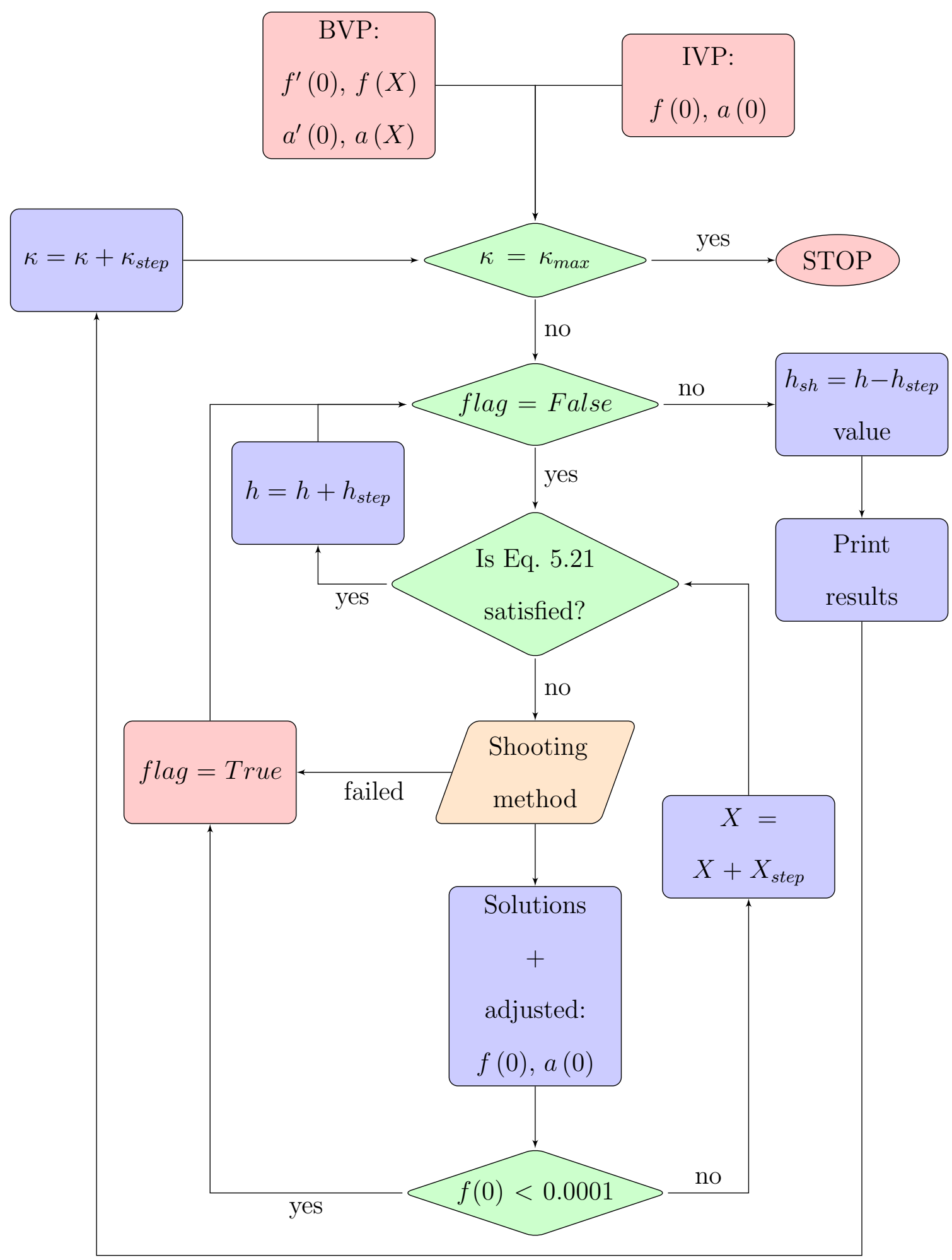

Figure 5.3. Flow chart representing the self-consistent numerical code to calculate $h_{s h}$. 

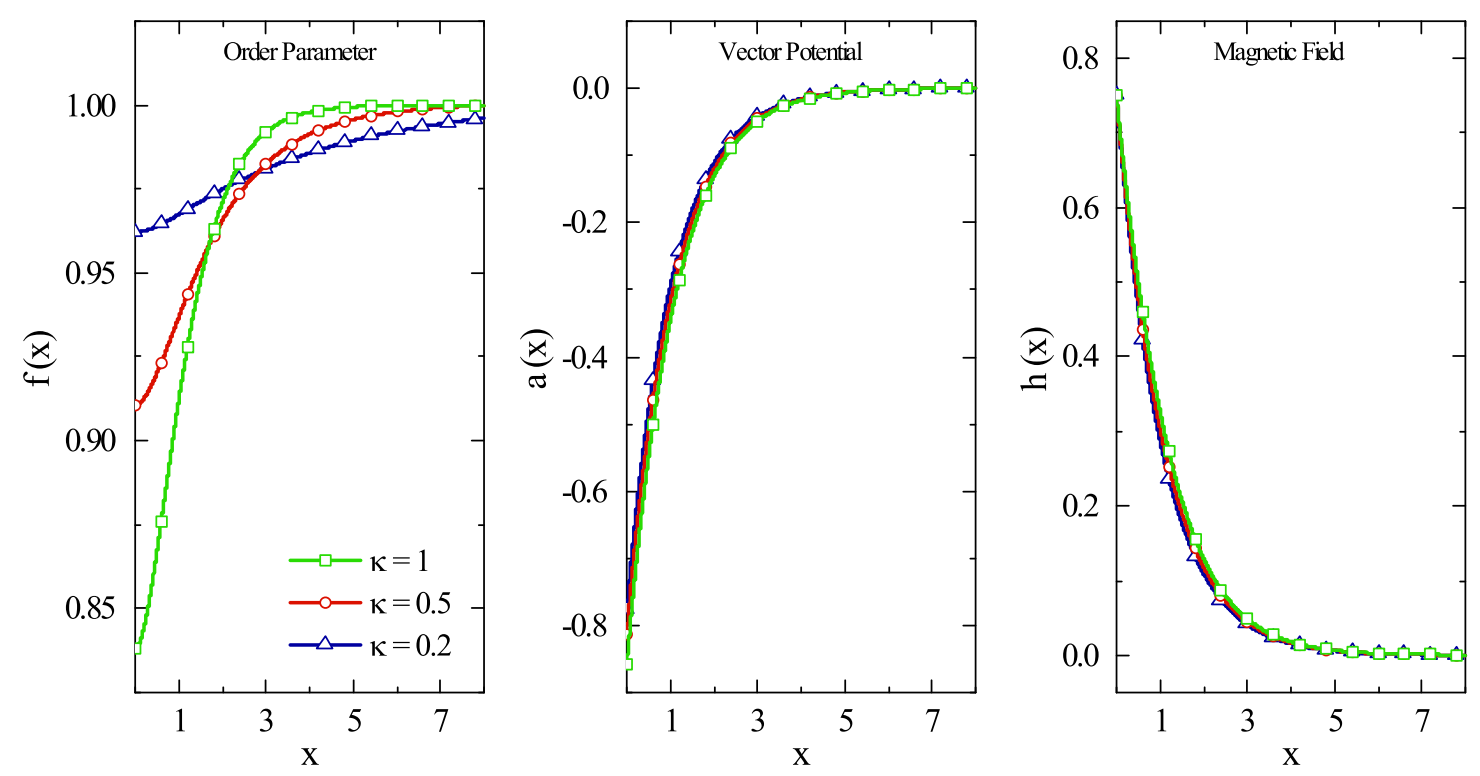

Figure 5.4. Numerical solution of the mono-dimensional Ginzburg-Landau equations for different values of $\kappa$ and applied field $h=0.75$.

flag on True, letting the code to print the superheating field value as before. The entire series of loops is then repeated till the maximum value of $\kappa_{\max }$ is reached.

In Figure 5.4, the numerical calculated order parameter, vector potential and magnetic field are plotted versus the normalized depth $x(=\operatorname{depth} / \lambda)$. Interesting to notice that for the same applied field $h$, the order parameter trend changes substantially assuming different $\kappa$ values. In particular, lower $\kappa$ values imply a more gradual variation of the order parameter, in agreement with a larger coherence length - the coherence length defines the typical dimension inside which the order parameter varies.

\subsection{Experimental Data and Numerical Calculations}

By meaning of the two numerical codes showed in the Appendix D, the normalized lower critical field $h_{c 1}=H_{c 1} /\left(\sqrt{2} H_{c}\right)$ and superheating field $h_{s h}=H_{s h} /\left(\sqrt{2} H_{c}\right)$ were calculated for increasing values of Ginzburg-Landau parameter, in the range $0.2 \leq \kappa \leq 3$. In Figure 5.5, the numerical values obtained are compared with pre- 


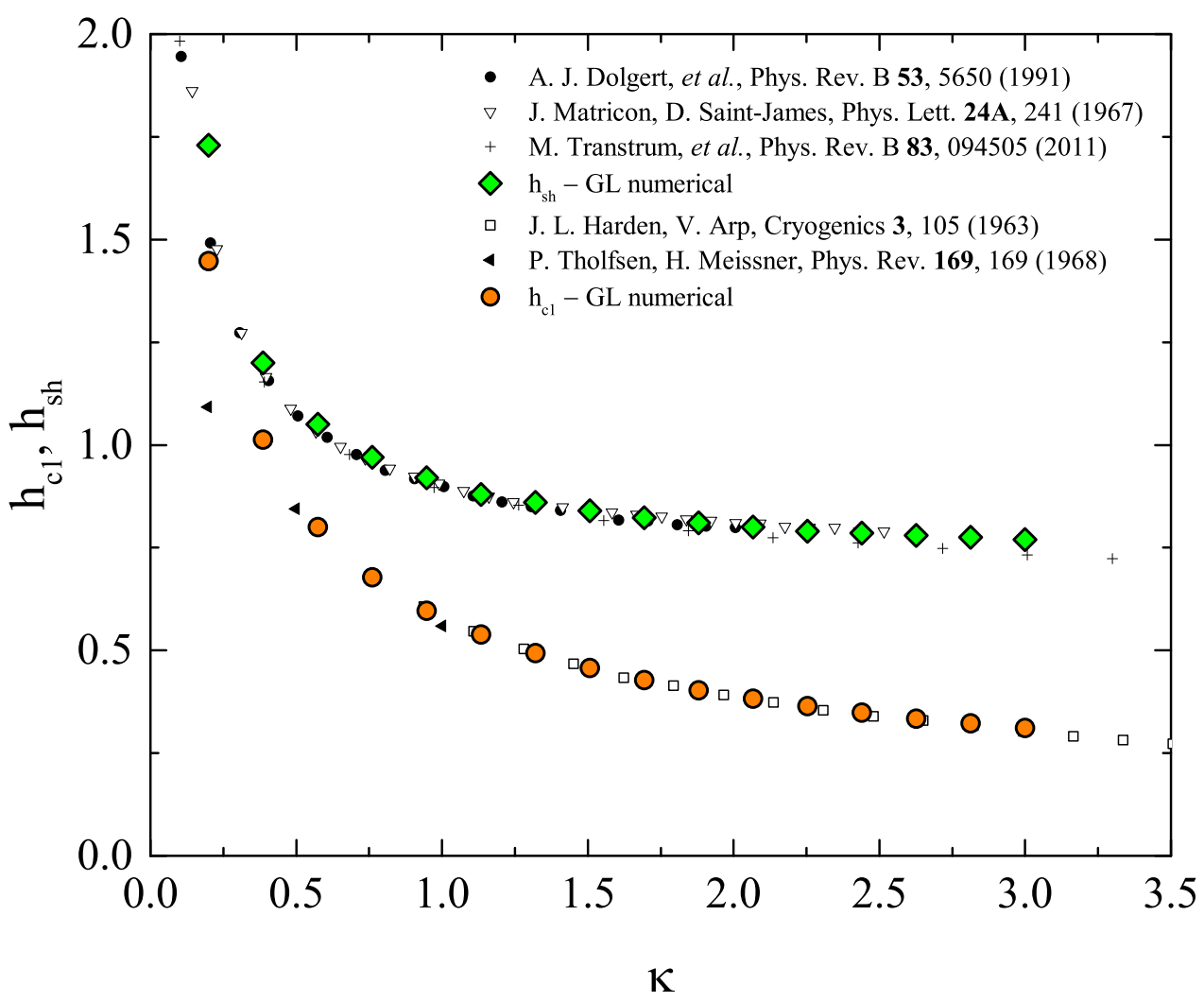

Figure 5.5. Numerical values of $h_{c 1}$ and $h_{s h}$ as a function of the Ginzburg-Landau parameter.

vious numerical calculations found in literature $[103,104,105,106,107]$. As it is clearly sown, the numerical results obtained are in good agreement with the previous calculations. The numerical solutions where fitted with two analytic formulas within the evaluation range $0.2 \leq \kappa \leq 3$ :

$$
\begin{aligned}
& h_{c 1} \simeq 0.58 \kappa^{-0.57} \\
& h_{s h} \simeq 0.72+0.18 \kappa^{-1}+0.004 \kappa^{-2}
\end{aligned}
$$

Several TESLA type [15] cavities were rf tested at the Fermi National Accelerator Laboratory's vertical test facility till their quench field. The complete list of cavities studied along with their magnetic peak field at the quench field and their thermal history are reported in Table 5.1. The mean free path values $(l)$ were calculated as described in $[10,75,76]$, while the Ginzburg-Landau parameters where 


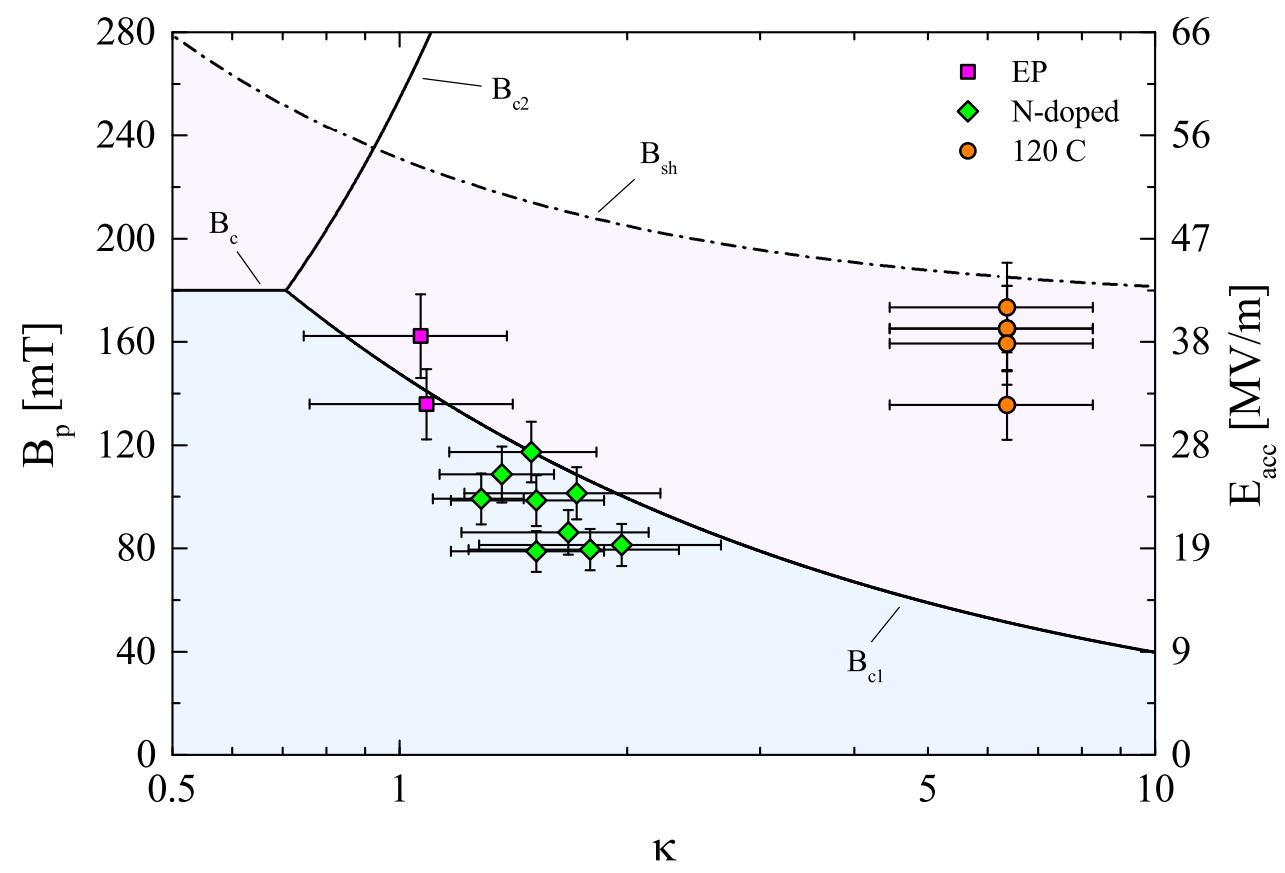

Figure 5.6. Experimental quench fields for different cavities as a function of $\kappa$ along with numerical values of lower critical and superheating fields.

calculated using the formulas:

$$
\kappa=\frac{\lambda}{\xi} \quad ; \quad \lambda=\lambda_{0} \sqrt{1+\frac{\xi_{0}}{l}} \quad ; \quad \xi=\left(\frac{1}{\xi_{0}}+\frac{1}{l}\right)^{-1}
$$

where $\xi_{0}=38 \mathrm{~nm}$ and $\lambda_{0}=39 \mathrm{~nm}[85]$.

In Figure 5.6, the experimental quench fields are reported as a function of the Ginzburg-Landau parameter along with the numerical simulations. The conversion $E_{a c c}$ to $B_{p}$ was done by means of the ratio defined in Section 3.1.3.

The Ginzburg-Landau theory is valid when the order parameter $\Psi$ is small - near $T_{c}$ for every field or near $H_{c 2}$ for every temperature-(see Section: 2.2), therefore it was assumed that by means of the empiric formula in Eq. 2.15, the lower critical field and superheating field values calculated near $T_{c}$ could be re-scaled at low temperatures $(T=2 \mathrm{~K})$.

The thermodynamic critical field $B_{c}$ value was then assumed equal to $180 \mathrm{mT}$ 
Table 5.1. Cavities studied. The values $\sigma_{B_{p}}, \sigma_{l}$ and $\sigma_{\kappa}$ are the errors associated to $B_{p}, l$ and $\kappa$ respectively.

\begin{tabular}{|c|c|c|c|c|c|c|c|c|}
\hline & Name & Treatment $^{\mathrm{a}}$ & $\begin{array}{l}B_{p} \\
{[\mathrm{mT}]}\end{array}$ & $\begin{array}{l}\sigma_{B_{p}} \\
{[\mathrm{mT}]}\end{array}$ & $\begin{array}{l}l \\
{[\mathrm{~nm}]}\end{array}$ & $\begin{array}{l}\sigma_{l} \\
{[\mathrm{~nm}]}\end{array}$ & $\kappa$ & $\sigma_{\kappa}$ \\
\hline \multirow{11}{*}{ 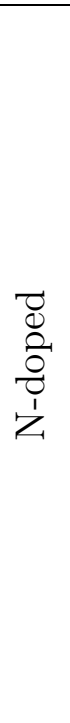 } & aes017_1 & $2 / 6 \mathrm{~min} \mathrm{~N}$-doped @ $800{ }^{\circ} \mathrm{C}+4 \mu \mathrm{m} \mathrm{EP}$ & 108.6 & 10.9 & 182 & 7 & 1.36 & 0.24 \\
\hline & aes009 & $2 / 6 \mathrm{~min} \mathrm{~N}$-doped @ $800{ }^{\circ} \mathrm{C}+5 \mu \mathrm{m} \mathrm{EP}$ & 98.6 & 9.9 & 128 & 5 & 1.52 & 0.35 \\
\hline & cbmm & $2 / 6 \mathrm{~min} \mathrm{~N}$-doped $@ 800^{\circ} \mathrm{C}+5 \mu \mathrm{m} \mathrm{EP}$ & 79.5 & 8.0 & 85 & 14 & 1.79 & 0.55 \\
\hline & aes011 & $2 / 6 \mathrm{~min} \mathrm{~N}$-doped $@ 800{ }^{\circ} \mathrm{C}+5 \mu \mathrm{m} \mathrm{EP}$ & 119.5 & 12.0 & - & - & - & - \\
\hline & aes017_2 $2^{b}$ & $2 / 6 \mathrm{~min}$ N-doped $@ 800{ }^{\circ} \mathrm{C}+7.5 \mu \mathrm{m}$ EP & 78.81 & 7.9 & 128 & 5 & 1.52 & 0.35 \\
\hline & aes025 & 2/6min N-doped @ $800{ }^{\circ} \mathrm{C}+15 \mu \mathrm{m} \mathrm{EP}$ & 99.2 & 9.9 & 238 & 3 & 1.28 & 0.17 \\
\hline & aes019_1 & 10min N-doped @ $800{ }^{\circ} \mathrm{C}+5 \mu \mathrm{m}$ EP & 117.3 & 11.7 & 134 & 7 & 1.49 & 0.33 \\
\hline & nr005 & $10 \mathrm{~min} \mathrm{~N}$-doped $@ 800{ }^{\circ} \mathrm{C}+5 \mu \mathrm{m}$ EP & 91.2 & 9.1 & - & - & - & - \\
\hline & $\operatorname{acc002}$ & $20 \mathrm{~min} \mathrm{~N}$-doped $@ 800{ }^{\circ} \mathrm{C}+5 \mu \mathrm{m} \mathrm{EP}$ & 81.4 & 8.1 & 70 & 10 & 1.97 & 0.69 \\
\hline & pav009 & $20 \mathrm{~min} \mathrm{~N}$-doped $@ 900{ }^{\circ} \mathrm{C}+5 \mu \mathrm{m} \mathrm{EP}$ & 101.4 & 10.1 & 93 & 10 & 1.72 & 0.50 \\
\hline & aes019_2 & $30 \mathrm{~min} \mathrm{~N}$-doped $@ 800^{\circ} \mathrm{C}+5 \mu \mathrm{m} \mathrm{EP}$ & 86.3 & 8.6 & 99 & 2 & 1.67 & 0.46 \\
\hline \multirow{2}{*}{ 届 } & pav007 & $\mathrm{EP}$ & 135.9 & 13.6 & 1000 & 100 & 1.09 & 0.32 \\
\hline & acc005_1 & $\mathrm{EP}$ & 162.3 & 16.2 & 1500 & 150 & 1.07 & 0.31 \\
\hline \multirow{5}{*}{ 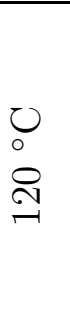 } & aes014 & $\mathrm{EP}+120^{\circ} \mathrm{C}$ & 135.6 & 13.6 & 16 & 1.6 & 6.36 & 1.90 \\
\hline & pav010 & $\mathrm{EP}+120^{\circ} \mathrm{C}$ & 173.4 & 17.3 & 16 & 1.6 & 6.36 & 1.90 \\
\hline & acc003 & $\mathrm{EP}+120^{\circ} \mathrm{C}$ & 165.2 & 16.5 & 16 & 1.6 & 6.36 & 1.90 \\
\hline & acc005_2 & $\mathrm{EP}+120^{\circ} \mathrm{C}$ & 165.2 & 16.5 & 16 & 1.6 & 6.36 & 1.90 \\
\hline & aes005 & $\mathrm{EP}+120{ }^{\circ} \mathrm{C}$ & 159.4 & 15.9 & 16 & 1.6 & 6.36 & 1.90 \\
\hline
\end{tabular}

a all the treatments (except for aes019_2, aes017_2 and acc005_2) are preceded by a $800{ }^{\circ} \mathrm{C}$ bake for 3 hours.

b preceded by a $1000{ }^{\circ} \mathrm{C}$ bake for 4 hours plus a $800{ }^{\circ} \mathrm{C}$ bake for 30 minutes. 
as experimentally measured for pure niobium in [108] at $2 \mathrm{~K}$, while the analytic expression used to plot the upper critical field $B_{c 2}$ is [33]:

$$
B_{c 2}=\sqrt{2} B_{c} \kappa
$$

The data reported in Figure 5.6 shows that statistically N-doped cavities quench in proximity of $B_{c 1}$, while cavities treated with the $120{ }^{\circ} \mathrm{C}$ mild baking are quenching always well above the lower critical field. Therefore $120{ }^{\circ} \mathrm{C}$ baked cavities are statistically overcoming $B_{c 1}$ and reaching the meta-stable Meissner state. In such state magnetic flux vortices would be stable in the superconductor bulk, since above $B_{c 1}$, but are instead confined outside because not energetically favorable to penetrate the surface, inasmuch below the superheating field.

Electro-polished cavities are instead quenching nearby the lower critical field, but since they are quenching in the high-field-Q-slope (HFQS) [99] regime, we cannot think to their limitation as surely introduced by the overcoming of one fundamental superconducting parameter ( such as $B_{c 1}$ or $B_{s h}$ ).

As explained in [54], the high-field-Q-slope might be introduced by the presence of nanometric niobium hydrides precipitates that are superconducting by proximity coupling [109] with the niobium matrix. When the field is increased above a certain level - the proximity break-down field - inversely proportional to the precipitate dimension, the proximity coupling is broken and niobium hydrides turned to normal-conducting. The HFQS can be then explained by the subsequent proximity coupling break-down of hydrides of different dimension with the increasing field. This mechanism though does not prevent the possibility that the proximity break-down may generate also quench. For this reason the electro-polished cavities will be not discussed further more.

5.5.1 Quench in Nitrogen-Doped Cavities. As explained in Section 5.2, if 


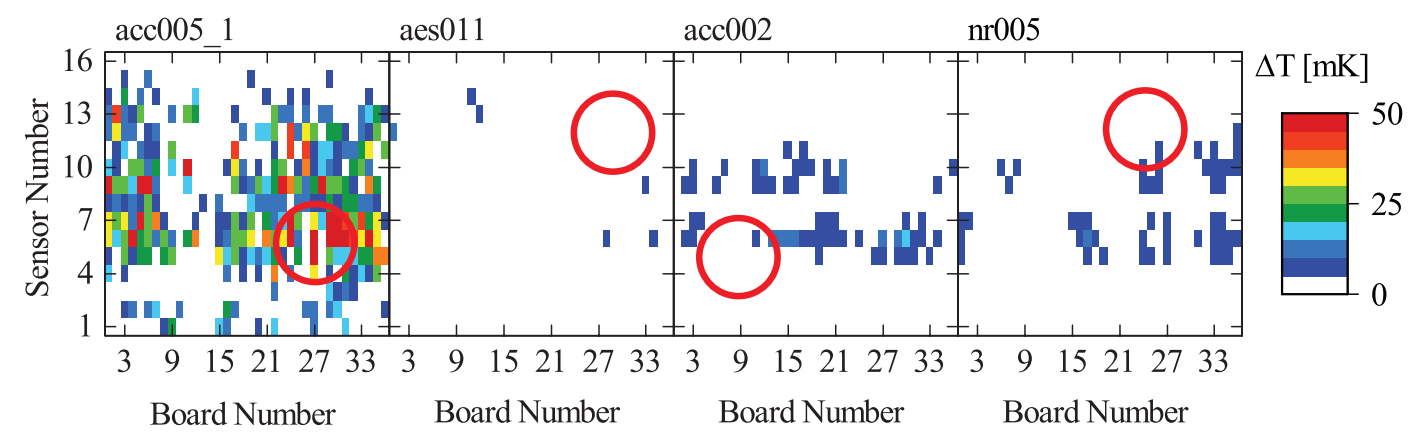

Figure 5.7. T-map acquired just before the cavity quench. The red circle shows the quench location.

defects are present at the cavity rf surface this latter may quench well below the field of first penetration because of thermal breakdown. The plot in Figure 5.6 should then be interpreted critically, and in order to discuss it correctly we need the further support of some T-map data.

It was observed that during continuous wave vertical rf measurements, Ndoped cavities were quenching without showing any warm up at the quench spot just before the quench event - preheating. Such effect is reported in Figure 5.7, where the T-maps acquired for some cavities (see Table 5.1) are reported.

The T-map measurements where performed for three N-doped cavities, and all of them did not show any kind of preheating - temperature increasing before the quench event - at the quench spot. Differently, if we observe the T-map acquired for a standard electro-polished cavity before the quench (acc005_1), the thermometers register the highest temperature among the whole cavity surface exactly were the quench is going to happen.

The preheating of cavity acc005_1 is shown also in Figure 5.8. As the field is increased (from 18.5 MV/m to $38.1 \mathrm{MV} / \mathrm{m}$ ), the thermometers shows a hot spot (red circle), that eventually turns into a quench (Figure 5.8f).

In Figure 5.9, this behavior is even more evident. For N-doped cavities the 

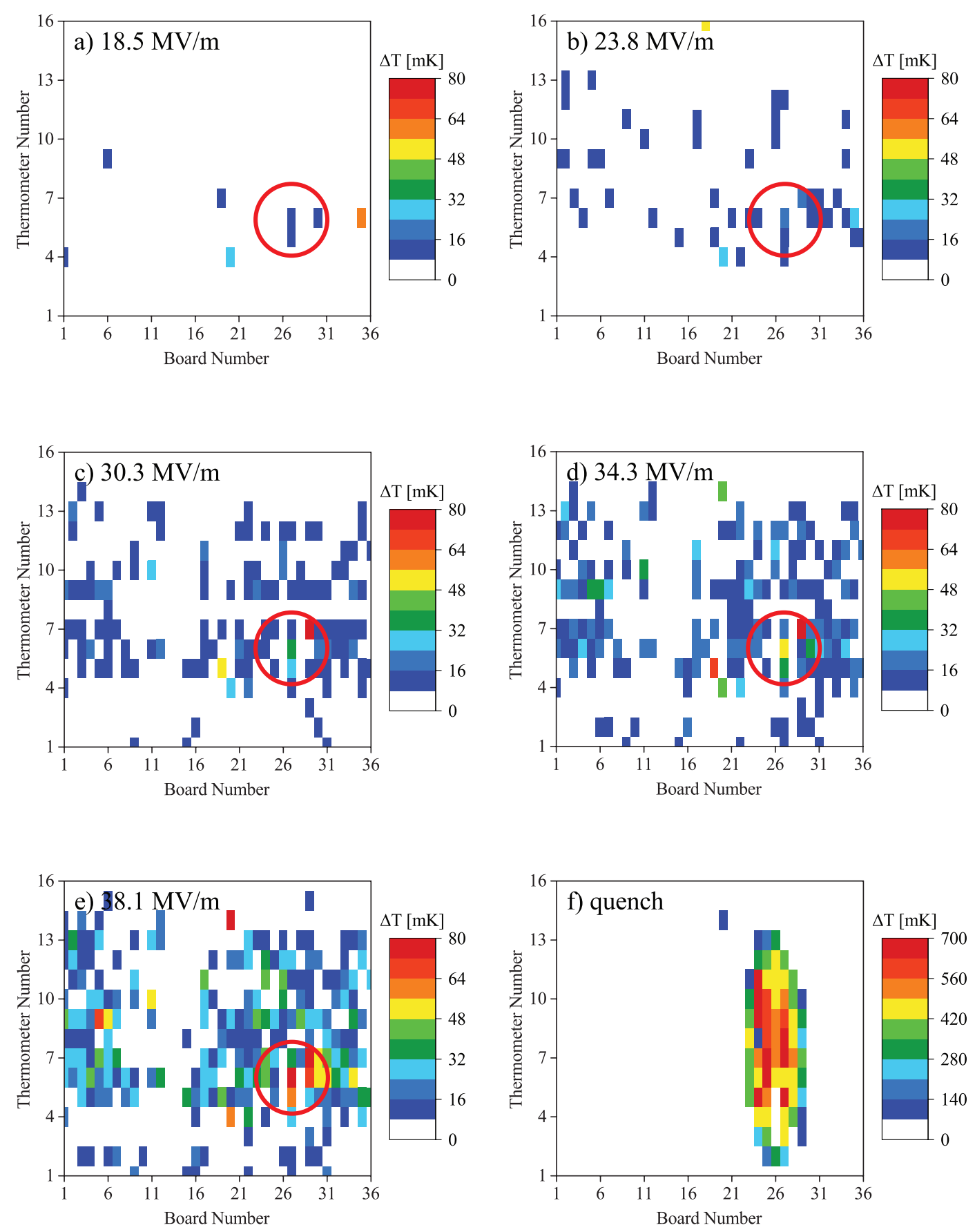

Figure 5.8. T-map data acquired during the first power rise of cavity acc005_1. Highlighted with the red circle the quench spot. 


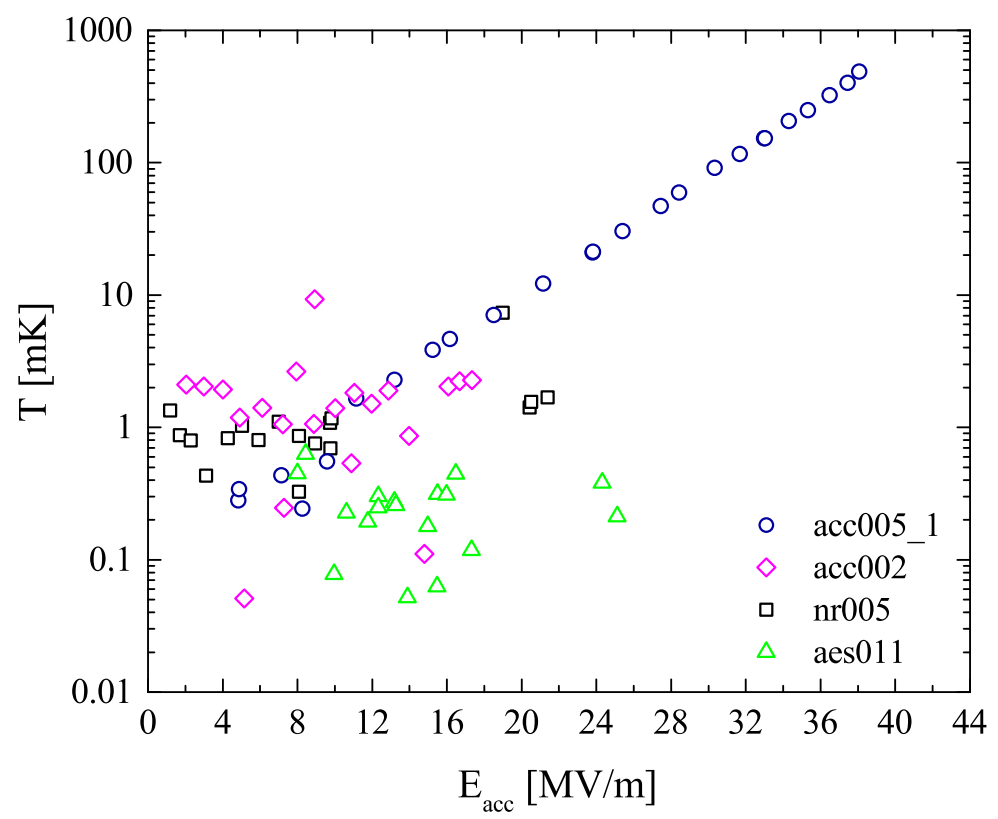

Figure 5.9. Temperature variation at the quench location as a function of the accelerating field.

temperature at the spot where the quench is going to happen remains constant as the rf field is increased in the cavity, while it increases approximately exponentially in the case of the EP cavity (acc005_1).

If no preheating is observed before the quench, we can directly rule out the thermal breakdown mechanism, since it assumes the quench as originated by the presence of a defect warmed up by the rf field. This means that differently from "standard" cavities (e.g. EP and $120^{\circ} \mathrm{C}$ baked cavities) that are usually limited by thermal breakdown, N-doped cavities are instead limited by a magnetic type of quench, i.e. magnetic field that penetrates the rf surface and ignites the quench event.

We can now again take into account Figure 5.6. Clearly, N-doped cavities are statistically quenching below $B_{c 1}$, and at the same time we know that such cavities are limited by a magnetic type of quench mechanism. We can therefore infer that in such cavities the quench usually happens because of magnetic field enhancement. 
The magnetic field is enhanced locally where superconducting asperities with high aspect ratio are present, allowing the magnetic field to penetrate earlier since locally higher than the field of first penetration.

Important to notice that, none of the N-doped cavities quenched above $B_{c 1}$, and their quench fields seem to follow the dependence of the lower critical field with the Ginzburg-Landau parameter. Such experimental finding suggests that probably for such cavities the meta-stable Meissner state for $B_{c 1}<B<B_{s h}$ is suppressed, and the cavities quench as soon as the condition of stability of vortices in the superconductor bulk is matched at $B_{c 1}(G \leq 0)$. Comparing the maximum quench field obtained with $\mathrm{N}$-doped cavities to those obtained with the $120^{\circ} \mathrm{C}$ baking, it appears clear that the latter can easily overcome the lower critical field and quench in the meta-stable Meissner state, even if their $B_{c 1}$ is lowered by the higher Ginzburg-Landau parameter value.

\subsection{Summary}

It this chapter the fundamental accelerating gradient limitation of SRF cavities was described. The lower critical field and the superheating field were calculated numerically form the Ginzburg-Landau equations for the $\kappa$ range between 0.2 and 3 . The experimental data acquired at $2 \mathrm{~K}$ for cavities with different $\kappa$ were compared to the numerical values of $B_{c 1}$ and $B_{s h}$.

The comparison suggests that N-doped cavities are statistically quenching below $B_{c 1}$ and therefore are not able to reach the meta-stable Meissner state. T-map measurements suggest also that N-doped cavities are quenching because of penetration of magnetic field at the quench spot. Since no preheating is observed at the location where the quench is going to happen, the conclusion is that the quench ignition mechanism is most likely the magnetic field enhancement. Such conclusion 
explains also why $\mathrm{N}$-doping cavities are seen usually quenching below $B_{c 1}$.

Different conclusions must be done for the $120{ }^{\circ} \mathrm{C}$ baked cavities. Statistically, such cavities are always quenching above the lower critical field in the meta-stable state. The vortex penetration is delayed, even if their lower critical field is lower than the N-doped cavities one.

In order to better understand the phenomenology underneath such different behavior we need to study the energetic of vortex penetration, which will be discussed in the following chapter (Chapter 6). 


\section{CHAPTER 6}

\section{ENHANCEMENT OF THE ACCELERATING GRADIENT}

\subsection{Chapter Overview}

As discussed in the previous chapter (Chapter 5), the fundamental limitation of superconducting accelerating cavities is related to the penetration of magnetic flux vortices in the superconductor. It was observed that particular thermal treatments can in principle promote the cavity in the so-called meta-stable Meissner state above $B_{c 1}$, and therefore enhance the accelerating gradient described.

The meta-stable Meissner state can in principle be described by means of an energetic argumentation on the vortex penetration at the rf surface. The main idea of this chapter is to take advantage of such energetic argumentation of vortex nucleation, to explain the different limitations encountered for cavities prepared with different surface finishing.

The vortex nucleation at the surface is discussed by means of the BeanLivingston energy barrier [110]. Initially, the calculation is carried out numerically from the Ginzburg-Landau equations for a uniform material with constant $\kappa$. A similar numerical approach is adopted for the second part of the calculations, where the Ginzburg-Landau equations are modified in order to account for a non-constant $\kappa$ profile. In particular, the calculation is performed in the scenario in which a dirty layer (high $\kappa$ ) is deposited or grown on top of a clean superconductor bulk (low $\kappa$ ).

The result of this latter suggests that when the dirty layer is present at the rf surface, the quench field of superconducting cavities can be enhanced. It was observed by low energy muon spin rotation (LE- $\mu \mathrm{SR}$ ) measurements [11], that $120^{\circ} \mathrm{C}$ baked cavities present different grades of dirtiness $(\kappa)$ at the very surface and in the bulk, that can be interpreted as the fingerprint of a dirty layer at the surface. 
The experimental results described in Chapter 5, that show $120{ }^{\circ} \mathrm{C}$ baked cavities quenching statistically always above $B_{c 1}$, can then be explained with the energetic argumentation on vortex penetration when a dirty layer is grown at the rf surface presented in this chapter.

The possible enhancement of the accelerating gradient by meaning of layered structures in accelerating cavities was initially introduced by A. Gurevich [111]. He showed that high $\kappa$ superconducting layers separated by insulating layers (SIS structure) deposited at the rf surface can in principle allow higher gradients. In the same direction T. Kubo $[112,113]$ and S. Posen et al. [114] explored in detail the behavior of the SIS structure. T. Kubo in particular, described also the "SS structure" [115, 113], i.e. a high $\kappa$ (dirty) superconducting layer on top of a low $\kappa$ (clean) bulk superconductor. He approached the problem in the high $\kappa$ approximation by meaning of the London equations, showing that the dirty layer can in principle enhance the accelerating field even if no insulating layer is present.

A similar situation is here presented, the "SS structure" is described as a $\kappa$ profile at the surface, and the problem is approached by solving numerically the Ginzburg-Landau equations for arbitrary $\kappa[116,117]$.

The dirty layer technology allows to reach high Q-factors at high gradients. N-doping (see Section 3.3) might be implemented to generate a dirty (doped) layer a the cavity surface, and therefore in principle to merge together the benefit of high gradients and high Q-factors. Some new results on $1.3 \mathrm{GHz}$ TESLA type cavities, that show high Q-factors at high gradients are presented. Such unprecedented results were achieved by implementing the new N-infusion treatment (see Section 3.3), which is demonstrated to generate a thin N-doped layer on top of a clean bulk.

\subsection{The Classic Bean Livingston Barrier}


The occurrence of the meta-stable Meissner state is related, though not equivalent, to the existence of an energy surface barrier to the magnetic flux penetration in the superconductor. Such surface barrier was for the first time described by C. P. Bean and J. D. Livingston [110] in dirty limit $(\lambda>>\xi$, or $\kappa>>1)$, and it represents the energy cost a vortex must spend in order to penetrate the surface of a type-II superconductor and reach its bulk.

Let us assume a semi-infinite type-II superconductor with constant $\kappa$ and perfect surface (without any irregularity, imperfection or defect), where a magnetic field is applied parallel to it. We can then describe the Bean-Livingston energy barrier in terms of Gibbs free energy density $g$ (in dimensionless notation $g=G /\left(n \mu_{0} H_{c}^{2} \lambda^{2}\right)$ ) for a vortex penetrating at the superconductor's surface.

The Gibbs free energy density $g$ can be defined as a function of the normalized coordinate $x$ (normalized with respect to $\lambda$ ) and composed by three terms:

i the interaction of the vortex with the magnetic field profile at the superconductor surface $\left(g_{f}(x)\right)$,

ii the interaction of the vortex with the surface $\left(g_{v}(x)\right)$ and

iii the Gibbs free energy density of a vortex far from the surface localized in the bulk of the material $\left(g_{\infty}\right)$.

The Gibbs free energy density then takes the general form:

$$
g(x)=g_{f}(x)+g_{v}(x)+g_{\infty},
$$

and it can be calculated by solving the integral of the total force acting on the vortex:

$$
g(x)=-\int f_{f}(x)+f_{v}(x) d x
$$


where $f_{f}(x)$ and $f_{v}(x)$ are the forces generated by the interaction of the vortex with the magnetic field profile and with the surface respectively $\left(f_{f}(x)\right.$ and $f_{v}(x)$ are forces per unit of length in dimensionless units $\left.f=\mu_{0} F /\left(B_{c}^{2} \lambda\right)\right)$.

6.2.1 Vortex - Field Interaction. Let us assume a superconducting surface in the plane $y z$, where a vortex penetrating from this surface is found at a distance $x$ from it, with magnetic flux along $\hat{y}$. The screening current $\mathbf{j}$ is instead flowing parallel to the surface along $\hat{z}$.

We can calculate the interaction force between the vortex in the material and the magnetic field profile at the surface by considering the Lorentz force per unit of length acting between the current density that screens the field and the flux quantum:

$$
\mathbf{F}_{\mathbf{f}}(x)=\mathbf{j}(x) \times \phi_{0} \hat{y}
$$

Taking advantage of the Maxwell equations and assuming the field profile $\mathbf{B}(x)=\left(0,0, B_{z}(x)\right)$, we can write:

$$
\begin{aligned}
\nabla \times \mathbf{B}(x) & =\mu_{0} \mathbf{j}(x) \\
& =\left(\frac{\partial B_{z}}{\partial y}-\frac{\partial B_{z}}{\partial z}\right) \hat{x}+\left(\frac{\partial B_{x}}{\partial z}-\frac{\partial B_{z}}{\partial x}\right) \hat{y}+\left(\frac{\partial B_{y}}{\partial x}-\frac{\partial B_{x}}{\partial y}\right) \hat{z} \\
& =-\frac{\partial B_{z}(x)}{\partial x} \hat{y}
\end{aligned}
$$

where $\partial B_{z} / \partial y=\partial B_{z} / \partial z=0$, since we assumed uniform field along $y$ and $z$. We can then define the screening current density at the vortex center location as equal to:

$$
\mathbf{j}(x)=-\frac{1}{\mu_{0}} \frac{\partial B_{z}(x)}{\partial x} \hat{y},
$$

and therefore the Lorenz force is:

$$
\begin{aligned}
\mathbf{F}_{\mathbf{f}}(x) & =-\frac{\phi_{0}}{\mu_{0}} \frac{\partial B_{z}(x)}{\partial x}(\hat{y} \times \hat{z}) \\
& =-\frac{\phi_{0}}{\mu_{0}} \frac{\partial B_{z}(x)}{\partial x} \hat{x}
\end{aligned}
$$




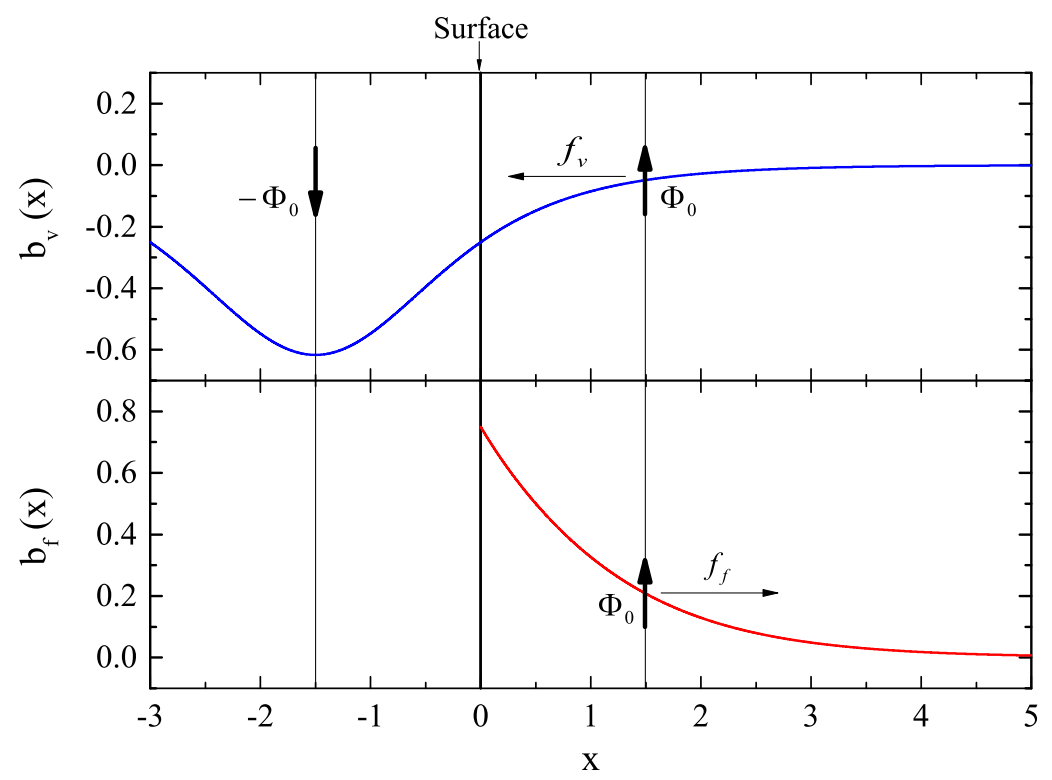

Figure 6.1. The top graph shows $b_{v}$ of the image vortex outside the superconductor $\left(-\Phi_{0}\right)$ that interacts with the vortex $\Phi_{0}$ inside the superconductor. The bottom graph shows instead vortex $\Phi_{0}$ inside the superconductor that interacts with the magnetic induction profile.

By meaning of the the normalization formulas that can be found in [103], the force per unit of length can be rewritten in terms of dimensionless quantities:

$$
\mathbf{f}_{\mathbf{f}}(x)=-\frac{4 \pi}{\kappa} \frac{\partial b_{f}(x)}{\partial x} \hat{x}
$$

where $\mathbf{f}_{\mathbf{f}}=\mu_{0} \mathbf{F}_{\mathbf{f}} / B_{c}^{2} \lambda, x$ correspond to the normalized quantity depth $/ \lambda$ and $b_{f}(x)$ is the magnetic induction profile decaying in the superconductor from the surface, calculated numerically from Eqs. 5.16 and $5.18\left(\right.$ the ratios $\left.b_{f}(x) \equiv h_{f}(x)\right)$.

Since the gradient of the magnetic induction $b_{f}(x)$ is negative, the force will be repulsive. The vortex is pushed inside the superconductor bulk and therefore moved away from the surface. The scenario just described is showed in the lower graph of Figure 6.1.

6.2.2 Vortex - Anti-Vortex Interaction. In order to calculate the interaction between the vortex and the superconductor surface, we can exploit the image vortex method [110]. We should consider an image vortex with same field profile, but 
different sign, outside the superconductor in the specular position of the real vortex. We can therefore calculate the interaction between the field of the image vortex and the flux quanta carried by the real vortex, assuming the origin of the system of coordinates to be centered at the image vortex center. As before, the interaction is mediated by the Lorentz force per unit of length:

$$
\mathbf{F}_{\mathbf{v}}(x)=\mathbf{j}(2 x) \times \phi_{0} \hat{y}
$$

Following what just done, but considering the magnetic induction profile of the image vortex $\mathbf{B}(2 x)=\left(0,0,-B_{z}(2 x)\right)$, we can write:

$$
\begin{aligned}
\nabla \times \mathbf{B}(2 x) & =\mu_{0} \mathbf{j}(2 x) \\
& =-\left[\left(\frac{\partial B_{z}}{\partial y}-\frac{\partial B_{z}}{\partial z}\right) \hat{x}+\left(\frac{\partial B_{x}}{\partial z}-\frac{\partial B_{z}}{\partial x}\right) \hat{y}+\left(\frac{\partial B_{y}}{\partial x}-\frac{\partial B_{x}}{\partial y}\right) \hat{z}\right] \\
& =-\frac{\partial B_{z}(2 x)}{\partial y} \hat{x}+\frac{\partial B_{z}(2 x)}{\partial x} \hat{y} .
\end{aligned}
$$

Since the vortex magnetic induction profile is assumed to be cylindrical symmetric (in first approximation also near the superconductor surface), the only spatial derivative equal to zero is $\partial B_{z} / \partial z=0$. We can then define the screening current at the vortex center location as equal to:

$$
\mathbf{j}(2 x)=-\frac{1}{\mu_{0}}\left(\frac{\partial B_{z}(2 x)}{\partial y} \hat{x}-\frac{\partial B_{z}(2 x)}{\partial x} \hat{y}\right)
$$

and therefore the Lorenz force is:

$$
\begin{aligned}
\mathbf{F}_{\mathbf{v}}(x) & =-\frac{\phi_{0}}{\mu_{0}}\left[\frac{\partial B_{z}(2 x)}{\partial y}(\hat{x} \times \hat{z})-\frac{\partial B_{z}(2 x)}{\partial x}(\hat{y} \times \hat{z})\right] \\
& =\frac{\phi_{0}}{\mu_{0}}\left[\frac{\partial B_{z}(2 x)}{\partial x} \hat{x}+\frac{\partial B_{z}(2 x)}{\partial y} \hat{y}\right] .
\end{aligned}
$$

Limiting the interaction only along $x$ and expressing the force per unit of length in terms of dimensionless quantities [103] we have:

$$
\mathbf{f}_{\mathbf{v}}(x)=\frac{4 \pi}{\kappa} \frac{\partial b_{v}(2 x)}{\partial x} \hat{x},
$$



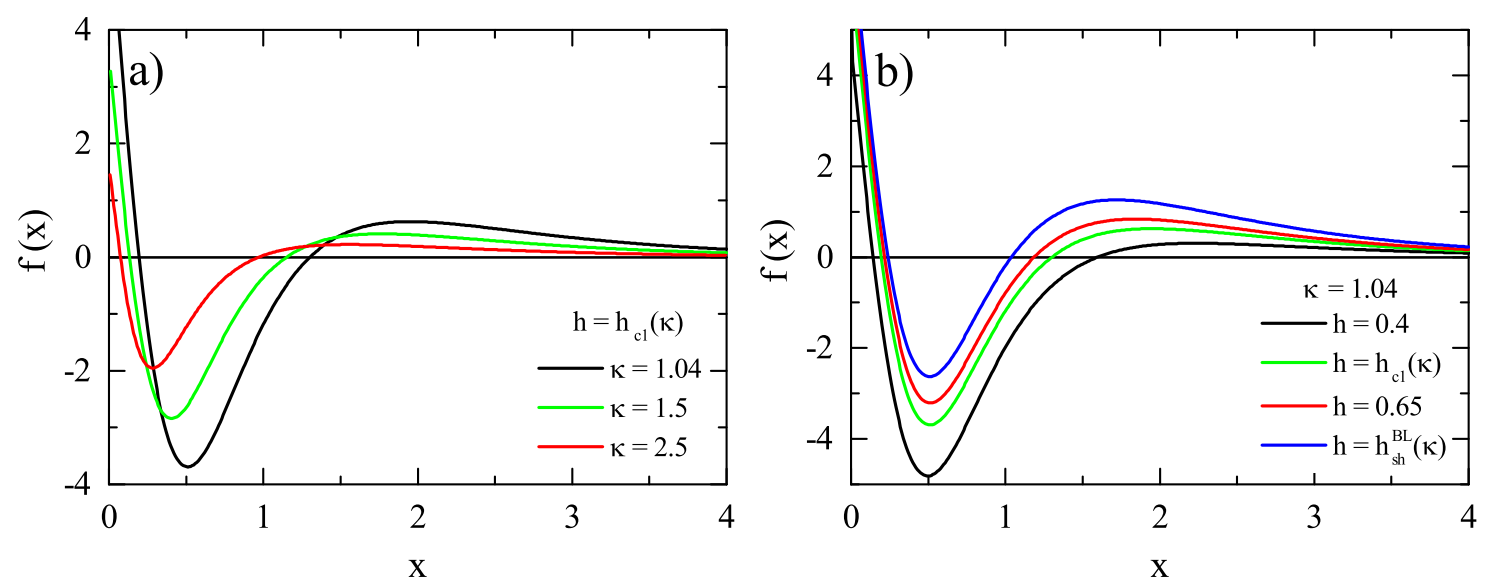

Figure 6.2. Total force acting on the vortex as a function of the normalized position $x$. In (a) the force is calculated for increasing $\kappa$ and field $h=h_{c 1}(\kappa)$ while in (b) for constant $\kappa=2.5$ but increasing field.

where $b_{v}(2 x)$ is the vortex magnetic induction profile calculated numerically by using Eqs. 5.9 and 5.11, and $x$ the normalized quantity depth $/ \lambda$.

Such interaction is attractive since the magnetic induction gradient along $x$ is negative. The vortex experiences an attractive force that pushes it towards the superconductor surface. The upper graph of Figure 6.1 shows the scenario just depicted, while in Figure 6.2 the total force acting on a vortex penetrating from the rf surface (Eq. 6.7 plus Eq. 6.12) is calculated as a function of the normalized position $x$.

6.2.3 Gibbs Free Energy Densisty. By meaning of Eq. 6.2, we can now calculate the Gibbs free energy density by substituting Eqs. 6.7 and 6.12:

$$
\begin{aligned}
g(x) & =-\frac{4 \pi}{\kappa} \int \frac{\partial b_{v}(2 x)}{\partial x}-\frac{\partial b_{f}(x)}{\partial x} d x \\
& =\frac{4 \pi}{\kappa}\left[b_{f}(x)-b_{v}(2 x)\right]+C
\end{aligned}
$$

where $C$ is a constant to be determined. If we assume the limit of $x$ tending to infinite, the terms dependent on $x$ goes to zero since both $b_{f}(x)$ and $b_{v}(2 x)$ are zero for $x \rightarrow \infty$. Therefore, we can define that $g_{\infty}=g(x \rightarrow \infty)=C$.

The Gibbs free energy density $G$ for a superconductor immersed in a magnetic 
field with non-interacting vortices located far from the surface is:

$$
G_{\infty}=n E-n \phi_{0} H
$$

By meaning of the definitions given in [103] and normalizing by the vortex areal density $n$, we get the dimensionless Gibbs free energy density $g_{\infty}$ :

$$
\begin{aligned}
g_{\infty} & =\epsilon-\frac{4 \pi}{\kappa} h \\
& =\frac{4 \pi}{\kappa}\left(h_{c 1}-h\right),
\end{aligned}
$$

where $g_{\infty}=G_{\infty} /\left(n \mu_{0} H_{c}^{2} \lambda^{2}\right)$ and $h_{c 1}=\epsilon \kappa / 4 \pi$.

The dimensionless Gibbs free energy density is then defined as:

$$
g(x)=\frac{4 \pi}{\kappa}\left[b_{f}(x)-b_{v}(2 x)+h_{c 1}-h\right]
$$

In Figure 6.3 the Gibbs free energy density simulated numerically from Eq. 6.16 is reported as a function of the normalized coordinate $x$.

Figure 6.3a shows the Gibbs free energy density calculated for different $\kappa$ values, with applied field equal to the lower critical field calculated at that particular $\kappa$. Important to notice that the barrier is present at the surface only, for increasing distances from the surface $g$ decreases till reaches zero in the material bulk. This behavior of $g$ is in perfect accordance with the condition that $g_{\infty}=0$ for $h=h_{c 1}$, as discussed previously in Section 5.3.

In Figure 6.3b, the Gibbs free energy is instead calculated for fixed $\kappa$ value, and different fields. For small fields values $\left(h=H / \sqrt{2} H_{c}=0.4\right)$, lower than $h_{c 1}$, the Gibbs free energy in the material bulk is larger than zero, meaning that vortices are not stable in the superconductor bulk. When $h=h_{c 1}$ vortices become stable in the material bulk, but in order to penetrate from the surface they still need to spend some energy defined by the barrier height, as just discussed for Figure 6.3a. 

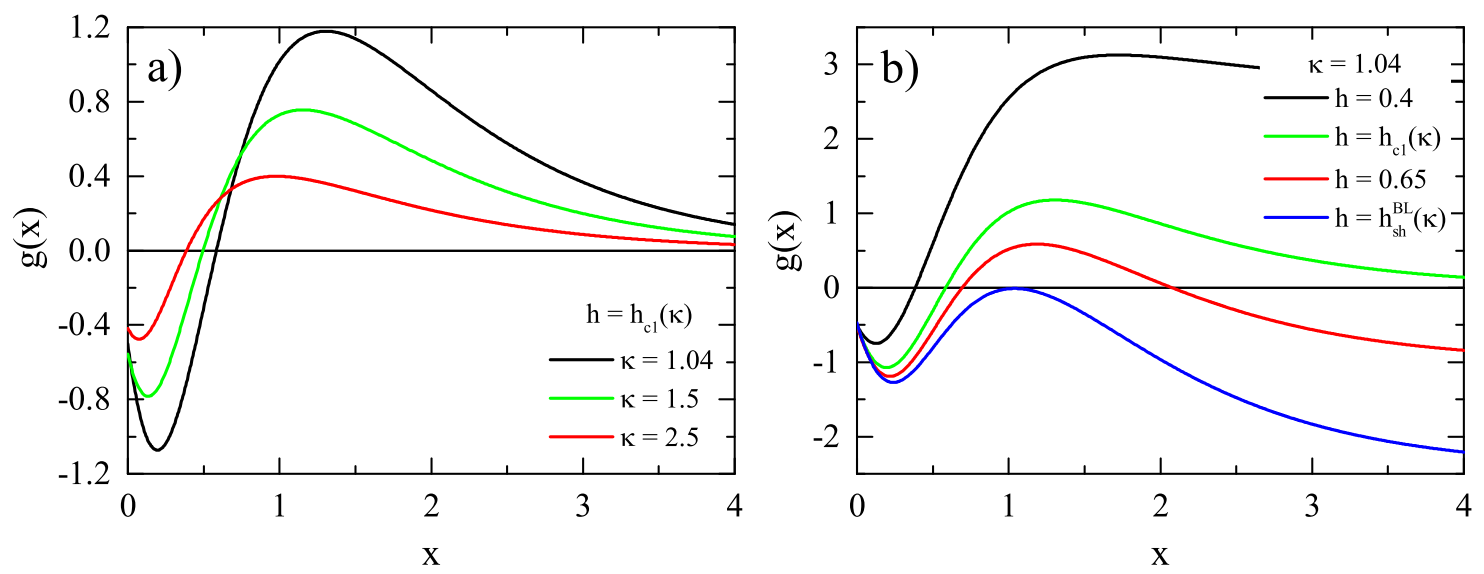

Figure 6.3. Gibbs free energy density as a function of the normalized position $x$. In (a) $g$ is calculated for increasing $\kappa$ and field $h=h_{c 1}(\kappa)$ while in (b) for constant $\kappa=2.5$ but increasing field.

When $h>h_{c 1}$ vortices are very stable in the material. From the pure bulk thermodynamics point of view, the superconductor is in the mixed state and $g<0$. On the other hand, the energy barrier at the surface is still present and in order to penetrate the vortex still has to spend some energy. Therefore, if no nucleation points are present (e.g. superficial defects), the superconductor will be in the meta-stable Meissner state where the flux is still confined outside the superconductor because of the presence of the surface.

If $h$ is increased even more, the energy barrier decreases in height till disappears when $h=h_{s h}^{B L}$ - the maximum of the Gibbs free energy density is equal to zero. Interesting to notice that such field has something in common with the superheating field $h_{s h}$, but it must not be confused with it. Indeed, for $\kappa=1.04$ we get $h_{s h}^{B L}=0.77$, that is smaller by a factor of 1.17 than $h_{s h}=0.9$, which is instead calculated by means of the Ginzburg-Landau theory. Such discrepancy is also discussed in [118], where the factor is claimed to be $\sqrt{2}$ for $\kappa=10$. The Bean-Livingston barrier is therefore a simple way to physically explain the presence of the meta-stable Meissner state, but only qualitative information on the superheating field can be obtained. On the other 
hand though, it contains good insights on the stability of the meta-stable Meissner state.

6.2.4 Disquisition on the Experimental Data. Let us consider again Figure 6.3a. As already said, it describes the Gibbs free energy density for $h=h_{c 1}$. This means that it corresponds to the energy barrier a vortex would see when $h=h_{c 1}$. Interesting to notice that as the Ginzburg-Landau parameter is increased, the barrier decreases, and the meta-stable Meissner state is destabilized. When defects are present at the surface (always in real surfaces), the surface barrier is partially or totally suppressed, and the penetration of vortices is favorable. If for the same surface condition (comparable defects number, roughness etc.) we consider also the dependence on $\kappa$, we would expect the vortex penetration to be even more favorable when $\kappa$ assumes larger values.

What we observe experimentally is reported in the previous chapter (Chapter 5) in Figure 5.6. We can assume that both $\mathrm{N}$-doped and $120{ }^{\circ} \mathrm{C}$ baked cavities have comparable surface goodness, with comparable roughness, number and type of defects, since both of them have an EP finishing. On the other hand though, N-doped cavities possess lower $\kappa$ than $120^{\circ} \mathrm{C}$ baked cavities, thus we should expect them to quench statistically at higher fields than $120{ }^{\circ} \mathrm{C}$ baked cavities. What we observe is instead the opposite, $120{ }^{\circ} \mathrm{C}$ baked cavities are statistically quenching at higher fields than N-doped cavities, even if their $\kappa$ is higher, and their meta-stable Meissner state destabilized by the smaller barrier.

Some low energy muon spin rotation $(\mathrm{LE}-\mu \mathrm{SR})$ measurements performed on cavites cut-outs [11], highlighted some huge differences of the magnetic induction profile in the superconductor as a function of different thermal treatments and superficial preparation. In Figure 6.4 such LE- $\mu \mathrm{SR}$ data is reported. From the point of view of the focus of this section, the most important information presented in the graph 


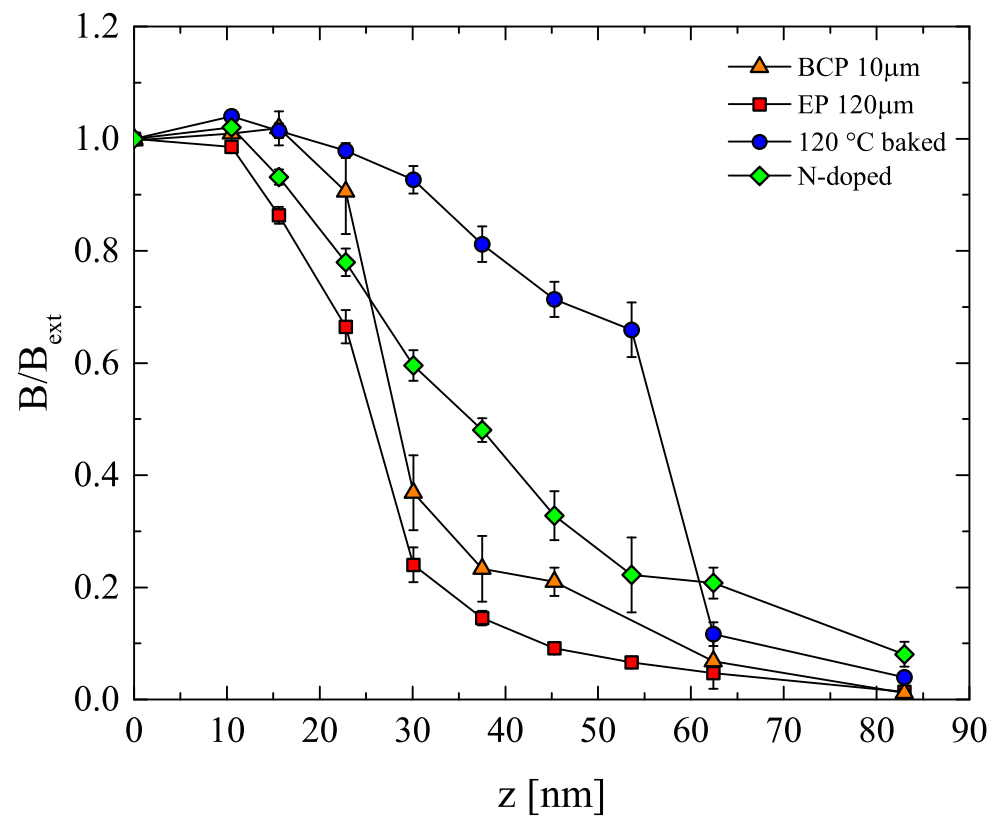

Figure 6.4. LE- $\mu \mathrm{SR}$ data reported in [11]. The N-doped data is courtesy of Dr. A. Romanenko

of Figure 6.4 is the Meissner screening of the $120{ }^{\circ} \mathrm{C}$ baked surface. As reported in [11], the Meissner screening of such surface is explainable only if the electron mean free path is assumed to vary inside the penetration depth (small at the very surface and large in the bulk), as if a dirty layer on the cavity surface was present. In the material bulk the mean free path of $120^{\circ} \mathrm{C}$ baked cavities is then comparable to the one of EP and BCP cavities. N-doped cavities (data courtesy of Dr. A. Romanenko) shows instead constant mean free path inside the penetration depth, meaning that the variation of the mean free path is negligible in the nanometric range.

Because of such variation of mean free path in the first namometers of material observed for $120{ }^{\circ} \mathrm{C}$ cavities, the description of the Bean-Livingston barrier till now adopted is not correct anymore. A more complicated scenario where the GinzburgLandau parameter varies inside the penetration depth must be considered in order to describe the higher quench field of such cavities with respect to the others.

\subsection{The Dirty Layer Effect on Vortex Nucleation}


Let us assume again a semi-infinite superconductor, where the normal to the surface $\hat{x}$ is directed towards the material bulk, the external magnetic field is applied along the $\hat{z}$ direction and the screening currents are flowing along the $\hat{y}$ direction. Differently than before, the Ginzburg-Landau parameter of such material is not constant, but it varies with an analytic form:

$$
\kappa(x)=-\frac{\kappa_{s}-\kappa_{b}}{1+e^{-\left(x-x_{0}\right) / c}}+\kappa_{s}
$$

which corresponds to a sigmoidal function, where $\kappa_{s}$ and $\kappa_{b}$ are the superficial and bulk Ginzburg-Landau parameters, $c$ is a constant that defines the steepness of the function (normally $c=0.018$ ) and $x_{0}$ corresponds to the sigmoid inflection point. Such function was chosen because represents a good approximation of a dirty layer of thickness $x_{0}$.

The physical dimension of the layer $d$ is then defined as:

$$
d=x_{0} \lambda \quad ; \quad \lambda=\lambda_{0} \sqrt{1+\frac{\xi_{0}}{l}},
$$

where:

$$
l=\frac{\xi_{0} \lambda_{0}^{2 / 3}}{\kappa_{m} \xi_{0}^{2 / 3}-\lambda_{0}^{2 / 3}} \quad ; \quad \kappa_{m}=\frac{k_{s}+k_{b}}{2}
$$

In first approximation, the forces acting on a vortex penetrating from the surface with a non-constant $\kappa$ profile can be calculated similarly the description implemented in the previous section.

6.3.1 Vortex - Field Interaction. The repulsive force the vortex experiences because of its interaction with the magnetic induction profile in the material (as done also in Subsection 6.2.2) is calculated as:

$$
\mathbf{f}_{\mathbf{f}}(x)=-\frac{4 \pi}{\kappa(x)} \frac{\partial b_{f}(x)}{\partial x} \hat{x}
$$

where $b_{f}(x)$ is calculated as $b_{f}(x)=a^{\prime}(x)$ from the Ginzburg-Landau equations (Eqs. 5.16 and 5.18) modified as follows in order to account also for the variation 
of $\kappa$ with $x$ :

$$
\begin{aligned}
& \frac{1}{\kappa^{2}(x)} f^{\prime \prime}-a^{2} f+f-f^{3}=0 \\
& \mathbf{a}^{\prime \prime}-f^{2} \mathbf{a}=0
\end{aligned}
$$

where the boundary conditions used are the same reported in Eq. 5.19.

6.3.2 Vortex - Image-Vortex Interaction. Following the reasoning reported also in Subsection 6.2.2, the attractive force is calculated as:

$$
\mathbf{f}_{\mathbf{v}}(x)=\frac{4 \pi}{\kappa(x)} \frac{\partial b_{v}(2 x)}{\partial x} \hat{x}
$$

where $b_{v}(2 x)$ is the magnetic induction of the image-vortex defined as $b_{v}(r)=a^{\prime}(r)+$ $(1 / r) a(r)$.

Firstly, in order to perform such calculation we should assume that the $\kappa$ profile is mirror-like symmetric with respect to the superconductor surface as well, i.e. the image-vortex "feels" the same $\kappa$ profile of the vortex in the material.

Secondly, the magnetic induction profile can be calculated from the cylindrically symmetric Ginzburg-Landau equations only if some approximations are made: i) both vortex and anti-vortex are assumed to be point-like objects, ii) the interaction between vortex and anti-vortex is defined only along the conjunction segment between them, and iii) because of (ii), the magnetic induction profile along the line of interaction is assumed to be not affected by the non-cylindrical symmetry of the $\kappa$ profile.

Under such assumptions the cylindrical symmetric GL equations (Eqs. 5.9 and 5.11) modified in order to account for such $\kappa$ profile are the following:

$$
\begin{aligned}
& f^{\prime \prime}+\frac{1}{r} f^{\prime}-\Gamma^{2}(r, x) f\left[f^{2}-1+\left(a-\frac{1}{\Gamma(r, x) r}\right)^{2}\right]=0 \\
& \mathbf{a}^{\prime \prime}+\frac{1}{r} \mathbf{a}^{\prime}-\frac{1}{r^{2}} \mathbf{a}-f^{2}\left(\mathbf{a}-\frac{1}{\Gamma(r, x) r} \hat{\theta}\right)=0,
\end{aligned}
$$




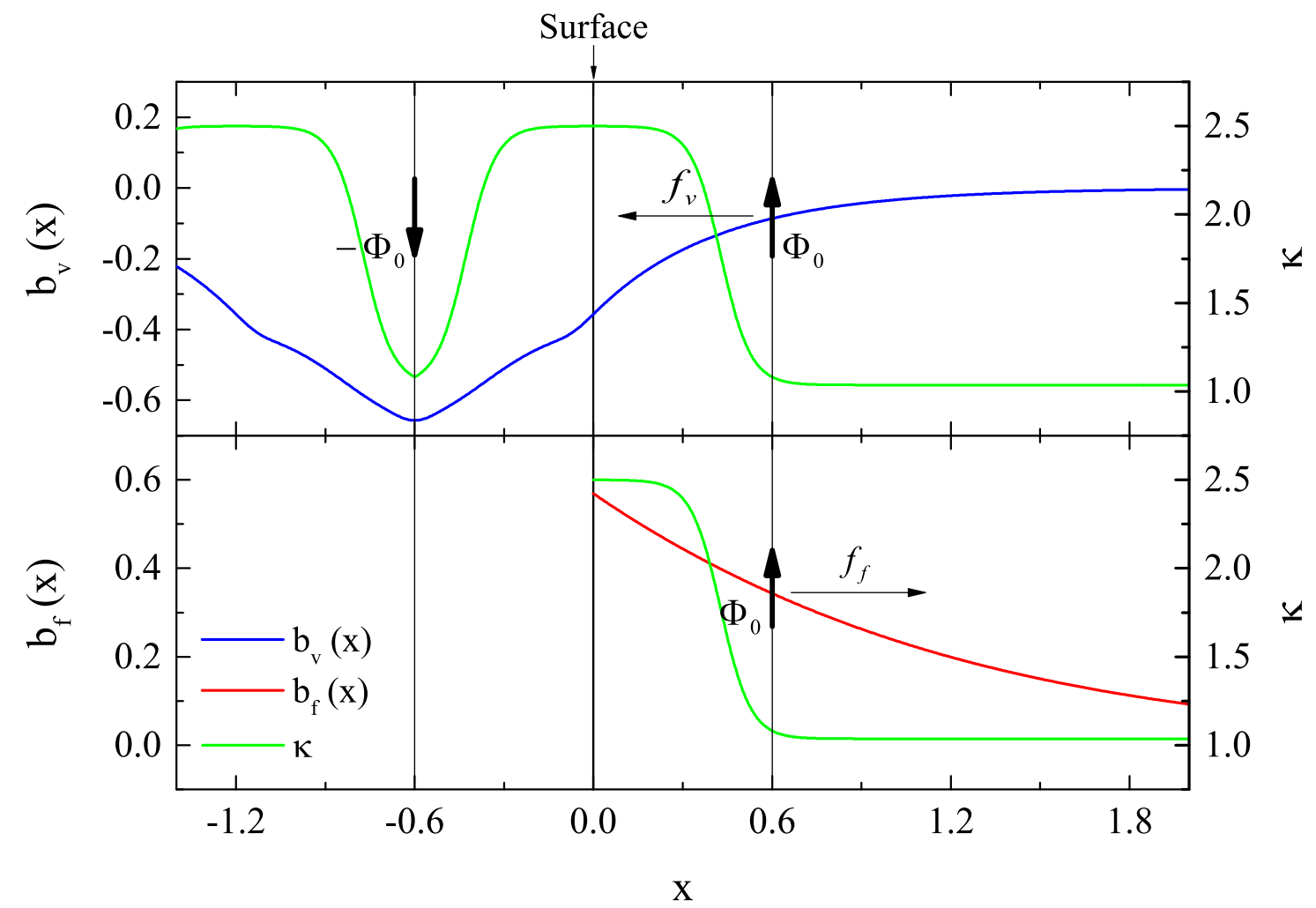

Figure 6.5. Magnetic induction profiles at the surface-lower graph-and of the antivortex - upper graph - for a non constant $\kappa$ profile and vortex position at $x=0.6$. The green lines correspond to the $\kappa$ profile in the two cases.

where $\Gamma(r, x)=\kappa(x-r)+\kappa(r-x)-\kappa_{s}$ is the $\kappa$ profile as seen by the image-vortex and $x$ is the vortex position with respect the surface. The boundary conditions used are the same assumed in Eq. 5.12, where the constant $\kappa$ is substituted by $\Gamma(R, x)$, with $R$ the domain extension.

In order to better visualize the problem, in Figure 6.5 the magnetic induction profiles of anti-vortex and applied field calculated by means of Eqs. 6.21 and 6.23, are plotted along with the Ginzburg-Landau parameter profile used.

As the vortex moves away from the rf surface, the $\kappa$ profile as seen by the antivortex from its position towards the vortex across the surface varies as a function of $x$. This means that the magnetic induction profile of the anti-vortex must be calculated 

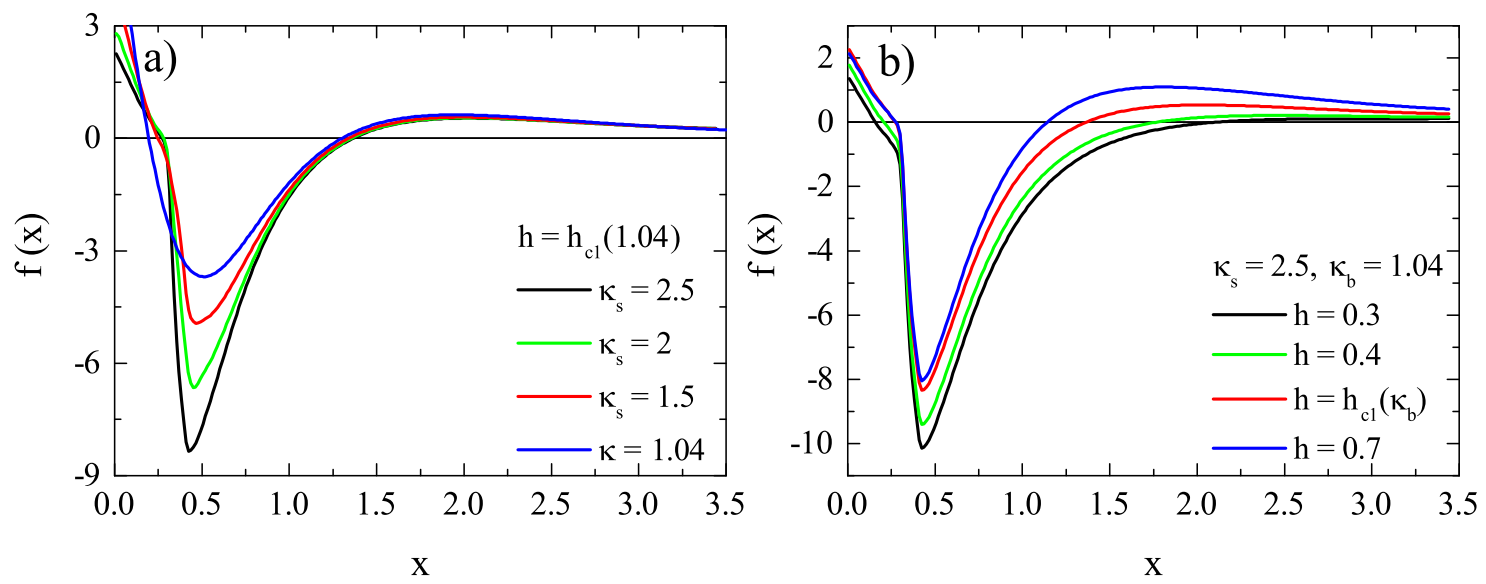

Figure 6.6. Total force acting on the vortex as a function of the normalized position $x$. In (a) the force is calculated for increasing superficial $\kappa_{s}$ and constant field $h=h_{c 1}\left(\kappa_{b}\right)$ while in (b) for constant $\kappa_{s}=2.5$ but increasing field. The layer thickness is fixed at $d=15 \mathrm{~nm}$ and $k_{b}=1.04$.

(form Eq. 6.23) for every vortex position $x$ at which we want to define the force. An example of forces calculated in such a way are reported in Figure 6.6, where the Ginzburg-Landau equations (Eqs. 6.21 and 6.23) where solved numerically for a $20 \mathrm{~nm}$ thick layer.

6.3.3 Total Force. In Figure 6.6a, the force as a function of the vortex position $x$ is reported for increasing values of $\kappa_{s}$ at the surface, calculated for constant applied field $h=h_{c 1}\left(\kappa_{b}\right)$. The larger $\kappa_{s}$, the sharper and pronounced is the attractive force peak. The attractive force is also enhanced compared to the constant $\kappa$ situation. The vortex experiences a larger attractive force towards the surface when the dirty layer is present, so that the vortex penetration is less favorable. On the other hand, the repulsive force peak remains more ore less constant.

In Figure 6.6b, the force is reported for different values of applied field, but same $\kappa$ profile. As the field increases the attractive peak decreases in height (and area) in favor of the repulsive peak growth.

In order to comprehend where this enhancement of attractive force cames 

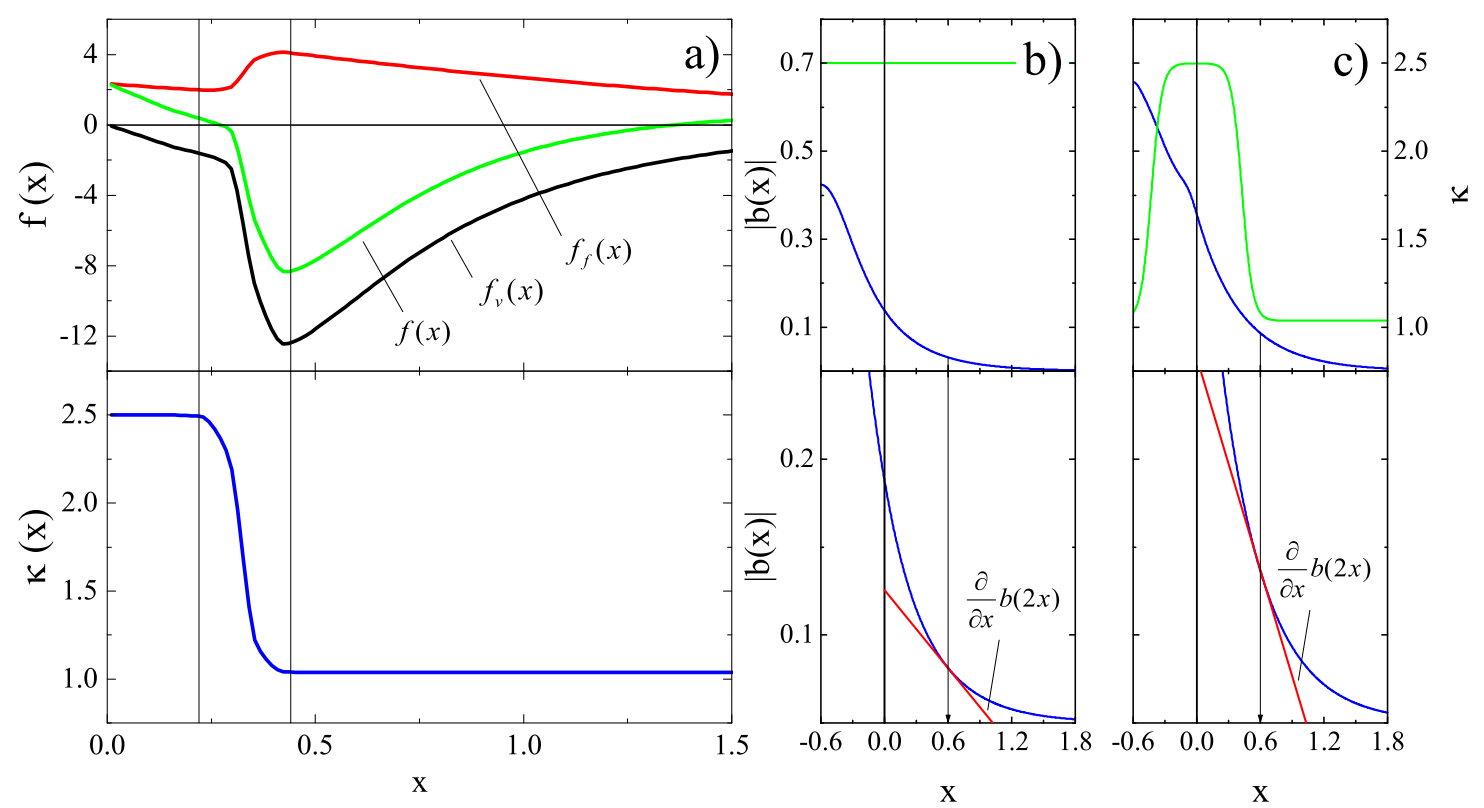

Figure 6.7. In (a) an example of force as a function of the position $x$ is plotted along with the $\kappa$ profile selected. In (b) and (c) the magnetic induction modulus of the image-vortex is plotted along with the $\kappa$ profile for constant $\kappa$ and dirty layer (non-constant $\kappa$ ) cases respectively.

from, we should consider Figure 6.7a, where the decomposition of the force $f(x)$ in attractive $f_{v}(x)$ and repulsive $f_{f}(x)$ forces is reported along with the $\kappa$ profile. Clearly the attractive force enhancement is generated by the change in GinzburgLandau parameter at the dirty layer boundary. In particular, we see that both the two contributions show an enhancement at the layer-bulk interface, but of opposite sign:

i the repulsive force is enhanced because the effective penetration depth $\lambda$ of the bulk is smaller than the superficial layer. In order to accommodate the abrupt variation in penetration depth, the screening currents at the layer-bulk interface must be enhanced, and the force acting on the vortex at the bulk-layer interface is larger,

ii the attractive force presents a peak generated by the perturbation of the magnetic induction distribution introduced by the layer. In Figure $6.7 \mathrm{~b}$ and $\mathrm{c}$, the magnetic 
induction profile is plotted as a function of the position when the $\kappa$ profile is constant and equal to 2.5 (b) and when the dirty layer is present (c). In both cases the vortex is localized at $x=0.6$. The two red lines represent the slope $\partial b_{v}(2 x) / \partial x$ in the two cases. When the dirty layer is present the slope $\partial b_{v}(2 x) / \partial x$ is larger, and therefore also the attractive force towards the surface.

6.3.4 Gibbs Free Energy Density. As it was done in the case of constant $\kappa$ (see Subsection 6.2.3), the Gibbs free energy density can be calculated numerically using the integral relation:

$$
\begin{aligned}
g^{*}(x) & =-\int f(x) d x \\
& =-\int \frac{4 \pi}{\kappa(x)}\left(\frac{\partial b_{v}(2 x)}{\partial x}-\frac{\partial b_{f}(x)}{\partial x}\right) d x \\
& =g_{s}^{*}(x)+g_{\infty},
\end{aligned}
$$

where the ${ }^{*}$ means that $g$ is defined up to an additive constant. In this case the pre-factor $4 \pi / \kappa(x)$ cannot be factorized out of the integral since dependent on $x$. The Gibbs free energy density associated to the interaction with the surface is here represented with $g_{s}^{*}(x)$.

Assuming that the dirty layer thickness $d$ is lower than the penetration depth at the surface $\left(d<\lambda_{s}\right)$, the surface layer can be treated as a perturbation on the magnetic induction profiles at the surface, while the bulk behavior remains totally independent from the surface condition. In such approximation, the calculation is substantially simplified and the Gibbs free energy at infinite $\left(g_{\infty}\right)$ can be calculated as before (Eq. 6.15) with $\kappa=\kappa_{b}$.

Since the integral in Eq. 6.24 must be determined numerically (in this case a trapezoidal rule was used), in order to assure that the Gibbs free energy density associated to the interaction with the surface is zero for $x \rightarrow \infty$, we need to define a 

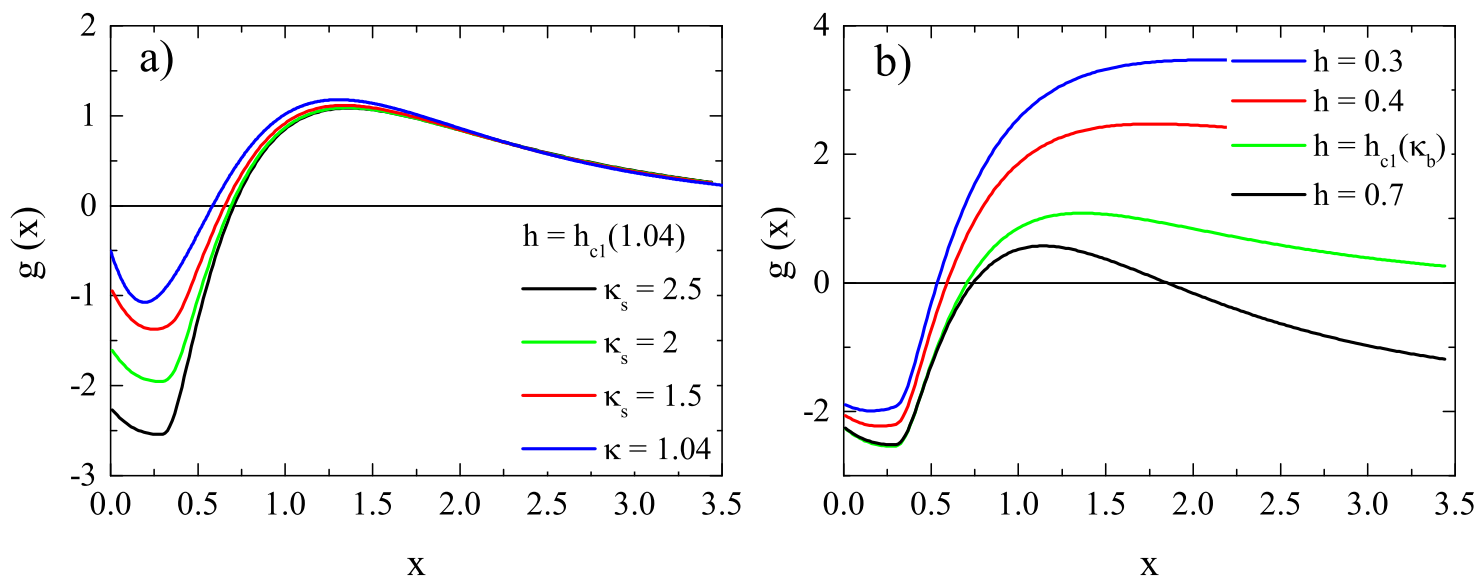

Figure 6.8. Gibbs free energy density as a function of the normalized position $x$. In (a) $g$ is calculated for increasing superficial $\kappa_{s}$ and constant field $h=h_{c 1}\left(\kappa_{b}\right)$ while in (b) for constant $\kappa_{s}=2.5$ but increasing field. The layer thickness is fixed at $d=15 \mathrm{~nm}$ and $k_{b}=1.04$.

new constant $C$ such that:

$$
g_{s}^{*}(x)=g_{s}(x)+C .
$$

The constant $C$ can be calculated similarly to $g_{\infty}$. Since $g_{s}(x)$ is associated to the total force of interaction with the surface $f(x)$, when $x \rightarrow \infty$, then both $f(x) \rightarrow 0$ and $g_{s}(x) \rightarrow 0$. Thus, if the solutions domain $X$ is large enough, the force and $g_{s}(x)$ values are so small that $g_{s}(X) \approx f(X)$ and the integration constant is:

$$
C=g_{s}^{*}(X)-f(X)
$$

It follows that the Gibbs free energy density is then calculated as:

$$
\begin{aligned}
g(x) & =g_{s}(x) * g_{\infty} \\
& =\left(g_{s}^{*}(x)-g_{s}^{*}(X)+f(X)\right)+\frac{4 \pi}{\kappa_{b}}\left(h_{c 1}\left(\kappa_{b}\right)-h\right),
\end{aligned}
$$

where $h_{c 1}\left(\kappa_{b}\right)$ is the bulk lower critical field defined as in Eq. 5.5. In Figure 6.8 the Gibbs free energy density calculation for a layer with thickness $d=15 \mathrm{~nm}$ and $\kappa_{b}=1.04$ are showed. 

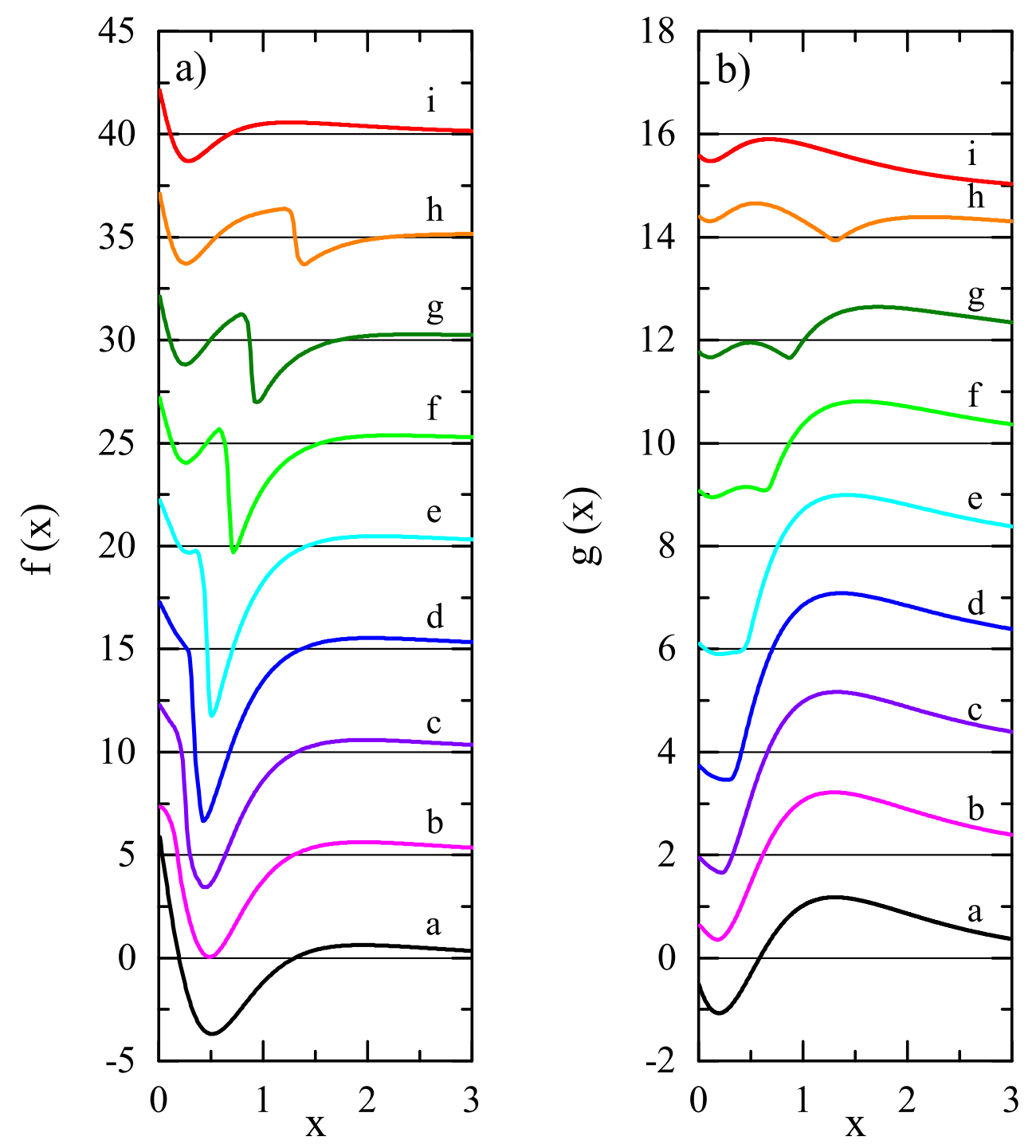

Figure 6.9. Numerical calculations of force (a) and Gibbs free energy density (b) for different dirty layer thicknesses. Curves $a$ and $i$ correspond to the limiting cases of layer with infinitely small and infinitely large thickness respectively. The plotted functions are shifted one respect to the other by $5 \mathrm{~cm}^{-1}$ in (a) and by 2 in (b). The horizontal lines represent the zero for every particular function. 
The graph in Figure 6.8a shows how the energy barrier evolves as a function of increasing $\kappa_{s}$ of the layer $(d=15 \mathrm{~nm})$. The blue curve is calculated in the cleanest limit with no layer at the surface. As the dirtiness of the layer is increased the barrier height with respect to zero is slightly decreased. The most noticeable variation is the initial positive slope of the curve that is increased by the presence of the dirty layer. Also, the absolute height of the barrier is increased since at the very surface the Gibbs free energy density assumes larger negative values as $\kappa_{s}$ increases. The stability of the meta-stable Meissner state is then increased with the introduction of the dirty layer since the $\Delta g$ the vortex must spend in order to penetrate is larger. Since all the curves in Figure 6.8a are calculated for $h=h_{c 1}\left(\kappa_{b}\right)$ and $d<\lambda_{s}$, the Gibbs free energy density tends to zero in the bulk of the material as expected.

In Figure 6.8b, the evolution of $g$ as a function of the applied field for a $15 \mathrm{~nm}$ layer with $\kappa_{s}=2.5$ and $\kappa_{b}=1.04$ is reported. The behavior of the Gibbs free energy is analogous to the one calculated with constant $\kappa$ profile showed in Figure 6.3b.

Of great importance is the dependence of the total force $f$, and of the Gibbs free energy density $g$, on the dirty layer thickness. Figure 6.9 shows the $f$ and $g$ trends for different dirty layer thicknesses, but same values of $\kappa_{s}=2.5$ and $\kappa_{b}=1.04$. In particular $a$ corresponds to the calculation performed for constant $\kappa=1.04$, the letters from $b$ to $h$ for dirty layers with $k_{s}=2.5, \kappa_{b}=1.04$ and thickness: $5,10,15$, 20, 30, 40 and $60 \mathrm{~nm}$, while $i$ for constant $\kappa=2.5$.

Figure 6.9a shows that as the dimension of the layer increases, the single peak found at about $x=0.5$ is split in two, where the first one will correspond to the slope of the Bean-Livington barrier at the superconductor surface, while the second one to the interface layer-bulk. If the layer is thinner enough (say less than $20 \mathrm{~nm}$ thick), the two peak are convoluted and cannot be distinguished. In the limit where the layer is large enough (several times the penetration depth $\lambda_{s}$ ), the behavior expected 
is equivalent to the one of a bulk with $\kappa=2.5$ (curve $i$ ).

In Figure 6.9b, the Gibbs free energy density is instead reported. As for the force case, up to a thickness of about $20 \mathrm{~nm}$ the effects of the two surfaces - surface and layer-bulk interface - are convoluted and the absolute Gibbs free energy density variation $\Delta g$ is increased. Above $20 \mathrm{~nm}, g$ starts to present two separate peaks. The smaller peak (the one related to the layer surface) has positive $g$ values when $d>40$ nm. Above such threshold value two different peaks with $g>0$ can be recognized.

Important to keep in mind that all such calculations were performed with constant field $h=h_{c 1}\left(\kappa_{b}\right)$ - the Gibbs free energy density $g$ should be less than zero if the material $\kappa$ is larger than $\kappa_{b}$. Indeed curve $i$, calculated for a constant $\kappa=2.5$ profile, is totally negative. This means that if the profile is constant with $\kappa=2.5$ and $h=h_{c 1}\left(\kappa_{b}\right)$ the superconductor is out of the Meissner state and vortices are stable in the bulk. On the contrary, if a dirty layer with $\kappa_{s}=\kappa=2.5$ is grown at the very surface (leaving the bulk with a low $\kappa_{b}$ ), the superconductor is still on the edge of the Meissner state, since its bulk energy is governed only by the bulk condition $\left(\kappa_{b}\right)$.

\subsection{Vortex Penetration Delay}

The findings just described are really important since imply that the presence of the dirty layer (if $d<\lambda_{s}$ ) does not modify the lower critical field $h_{c 1}$ of the material. In other words, the Meissner state can survive up to the lower critical field of the bulk independently on the surface condition.

Such interesting effect introduced by the dirty layer can be clearly seen in Figure 6.10. As showed, since the dirty layer is treated only as a perturbation on the magnetic induction profiles $\left(d<\lambda_{s}\right)$, the Gibbs free energy density at infinite corresponds to $g_{\infty}\left(\kappa_{b}\right)$. If we compare the results obtained for the constant $\kappa=2.5$ profile to those obtained for layers of thickness $d=15,30$ and $60 \mathrm{~nm}$, the practical 


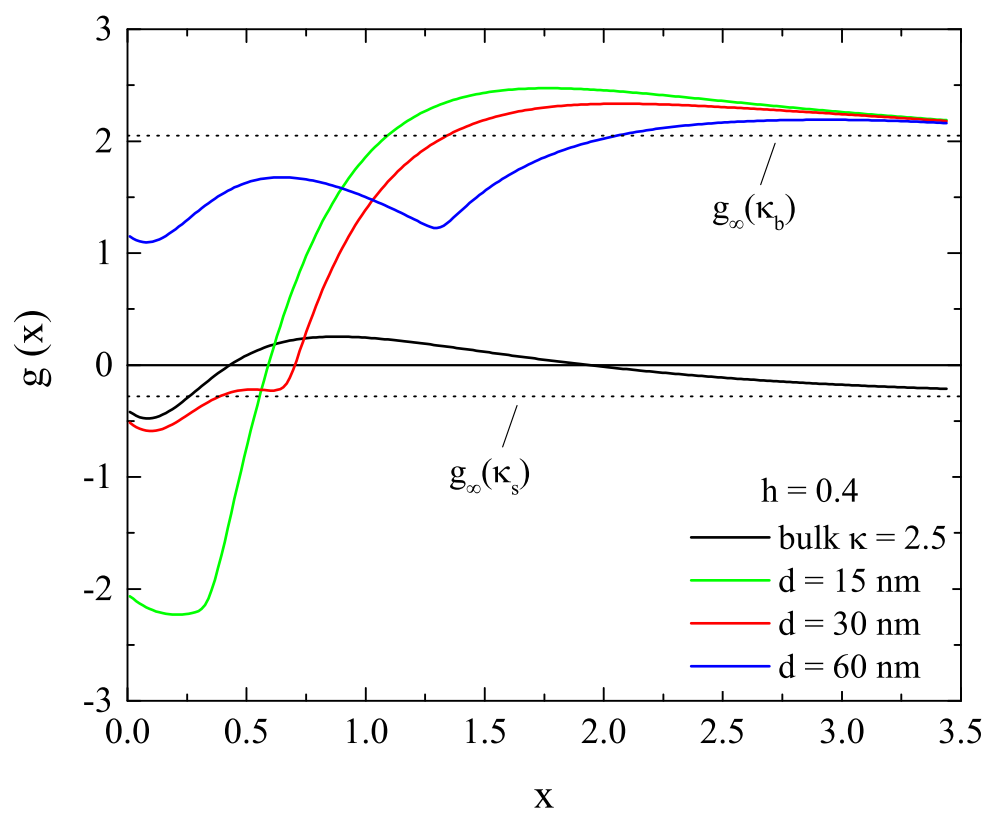

Figure 6.10. Comparison between the Gibbs free energy density calculated for a constant $\kappa=2.5$ profile and a for non-constant profiles with layers having $\kappa_{s}=2.5$, $\kappa_{b}=1.04$ and various thicknesses when $h=0.4>h_{c 1}\left(\kappa_{s}\right)$.

outcome of such finding is clear. When $\kappa$ is constant and $h>h_{c 1}(\kappa)$, vortices are stable in the material bulk and the superconductor is in the meta-stable Meissner state. If instead the dirty layer is present, even if $h>h_{c 1}\left(\kappa_{s}\right)$, the Meissner state is preserved up to $h=h_{c 1}\left(\kappa_{b}\right)$, i.e. the minimum field at which vortices are stable in the superconductor is enhanced compared to what you would expect by considering only the superficial $\kappa_{s}$.

In practical words, the probability of quenching the cavity at $h_{c 1}\left(\kappa_{s}\right)$ because of flux penetration is decreased when the dirty layer is present at the surface. In real surfaces where defects are always present (let us assume only geometric superconducting defects, thermal breakdown is not considered), the Bean-Livingston barrier is weakened or totally suppressed. Thus, we should expect a generous part of constant $\kappa$ profile cavities (e.g. nitrogen-doped) to quench at the lower critical field or below. On the contrary, cavities that do not possess a constant $\kappa$ profile (e.g. $120^{\circ} \mathrm{C}$ 

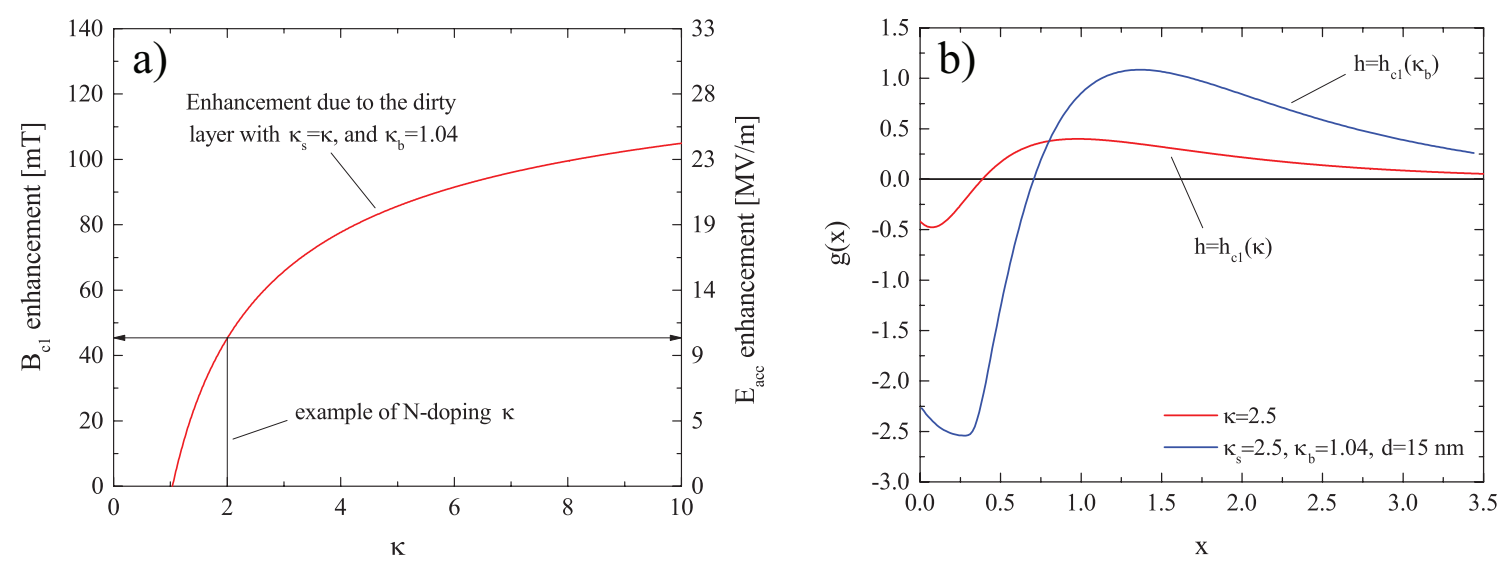

Figure 6.11. In (a) enhancement of the lower critical field as a function of $\kappa$ when the dirty layer is assumed. In (b) comparison between the Gibbs free energy density for constant $\kappa$ and dirty layer cases when the vortex starts to be stable in the material bulk $\left(g_{\infty}=0\right)$.

baked) should be able to reach fields above $h_{c 1}\left(\kappa_{s}\right)$ even if the same type of defects are present at the surface, since their Meissner state survives up to $h_{c 1}\left(\kappa_{b}\right)>h_{c 1}\left(\kappa_{s}\right)$. Such effect is indeed experimentally observed in Figure 5.6 in Chapter 5.

In Figure 6.11a the lower critical field enhancement (calculated with $B_{c}=$ $180 \mathrm{mT}$ [108]) when the dirty layer is grown at the superconductive surface is reported. In the graph, $\kappa$ represents either the $\kappa$ of the dirty layer at the surface $\left(\kappa_{s}\right)$, or the constant $\kappa$ of the homogeneous superconductor. Assuming a typical $\kappa$ value for a Ndoped cavity $(\kappa=2)$ the expected minimum enhancement of the accelerating gradient is about $10 \mathrm{MV} / \mathrm{m}$.

The dirty layer should also partially stabilize the meta-stable Meissner state, since the $\Delta g$ seen by the vortex at the very surface increases with the layer dirtiness $\left(\kappa_{s}\right)$, as discussed in Subsection 6.3.4. Moreover, when vortices are stable in the superconductor bulk $\left(g_{\infty}=0\right)$, the energy barrier to vortex penetration is higher, as shown in Figure 6.11b. Non-constant $\kappa$ profile cavities, as $120^{\circ} \mathrm{C}$ baked, should then in principle have more probability of operate in the meta-stable Meissner state above $h_{c 1}\left(\kappa_{b}\right)$, as also observed experimentally and shown in Figure 5.6. 


\subsection{Nitrogen Infusion for High $Q_{0}$ at High Gradients}

A series of experiments were performed in order to push N-doped cavities to higher gradients exploiting the predictions of the model just described. In order to create such dirty layer at the rf surface of the cavity, the N-infusion treatment was adopted (see Section 3.3).

Such mild baking in nitrogen atmosphere produces a superficial concentration of nitrogen similar to N-doping, but the trend of the concentration profile with the depth is totally different. In N-doped surfaces the concentration profile can be considered almost constant within several penetration depths $\lambda$ (about $1 \mu \mathrm{m}$ or more). $\mathrm{N}$-doped cavities can then be considered as constant $\kappa$ materials.

On the other hand, N-infused surfaces present interstitial nitrogen only in the first tents of nanometers, i.e. a dirty layer of thickness comparable or smaller than the superficial penetration depth $\left(\lambda_{s} \approx 60 \mathrm{~nm}\right)$. Such difference in concentration profile is reported in Figure 6.12. The secondary ion mass spectroscopy profile for nitrogen is reported for a N-doped cavity (20min with 25 mTorr of nitrogen) and for a N-infused witness sample $\left(160{ }^{\circ} \mathrm{C}\right.$ with 25 mTorr for $\left.48 \mathrm{~h}\right)$.

In Figure 6.13, the quench data for N-doped cavities and N-infused cavities are shown. As explained in [10], when the impurities concentration varies rapidly at the surface as in the case of $120^{\circ} \mathrm{C}$ baked or $\mathrm{N}$-infused cavities, the mean free path (and therefore $\kappa$ ) cannot be extrapolated from the variation of the frequency with temperature. In such situation, the most direct method to extrapolate the electron mean free path is by fitting the magnetic induction profile measured by LE- $\mu$ SR. Since such technique is not available at Fermilab, the exact $\kappa_{s}$ value of N-infused cavities is unknown. The $\mathrm{N}$-infused cavity superficial $\kappa_{s}$ was therefore assumed to be in the $\kappa$ range of $\mathrm{N}$-doped cavities, since the nitrogen superficial concentration is of 


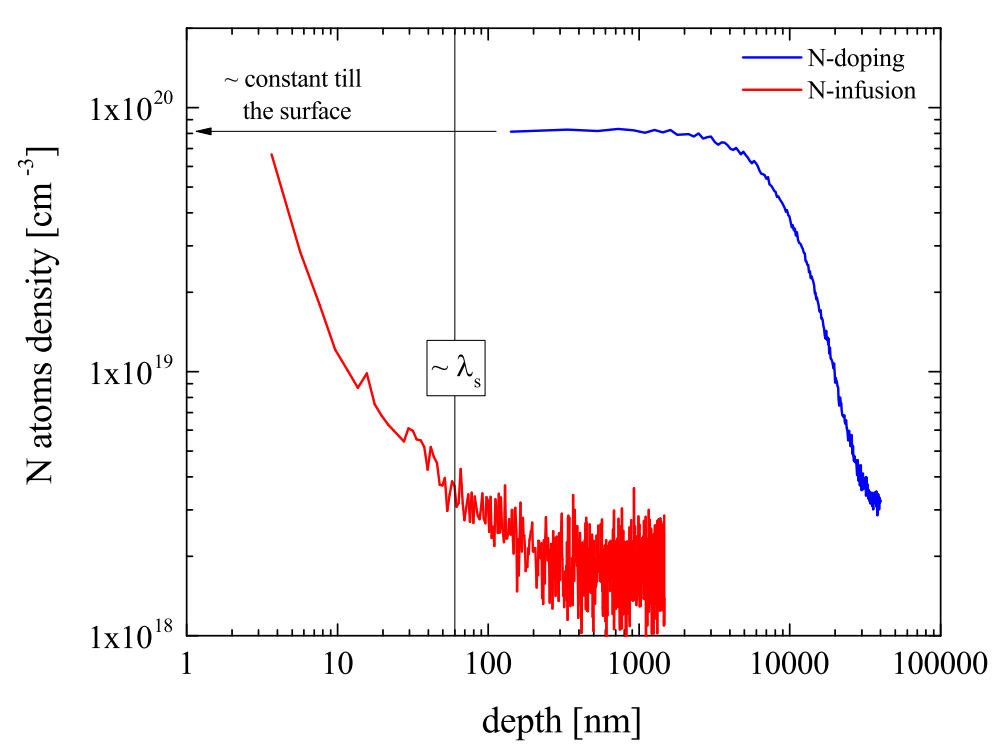

Figure 6.12. SIMS profile for nitrogen of a N-doped cavity cut-out, and of a N-infused witness sample.

the same order of magnitude for the two treatments.

The dotted lines in Figure 6.13 show $B_{s h}$ and $B_{c 1}$ of a bulk superconductor with constant $\kappa$ (like $\mathrm{N}$-doped cavities). The blue area underneath $B_{c 1}(\kappa)$ and the sum of pink and green areas show respectively the extension of the Meissner state and of the meta-stable Meissner state for constant $\kappa$ cavities. When the dirty layer with thickness $d<\lambda_{s}$ is introduced, the value of $B_{c 1}$ is constant and dependent only on $\kappa_{b}$ (in this case assumed to be 1.04), as described previously in Section 6.4. The Meissner state is now represented by the sum of blue and green areas, while the meta-stable Meissner state by the pink area alone.

As expected, N-infused cavities are quenching above the bulk lower critical field value, since the dirty layer is shifting the Meissner state up to higher fields. The meta-stable Meissner state is also stabilized by the dirty layer thanks to a larger $\Delta g$. Indeed $\mathrm{N}$-infused cavities can sustain fields well above $B_{c 1}\left(\kappa_{s}\right)$. Anyway, no speculations on the ultimate gradient limitations of such cavities can be done yet. The superheating field for such layered surfaces is not yet calculated numerically from 


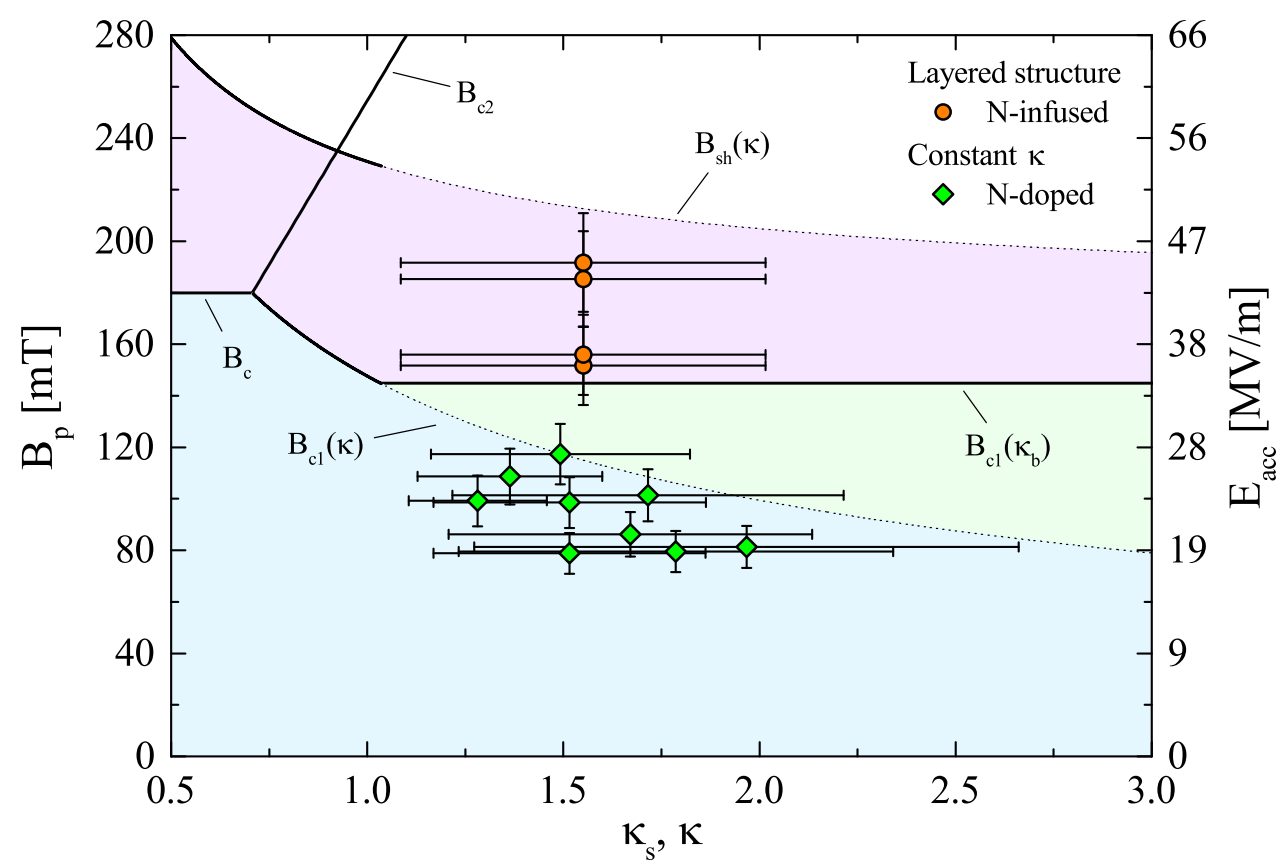

Figure 6.13. Quench data for N-doped and N-infused cavities as a function of $\kappa$ plotted along with the numerical calculations of the critical fields. The dotted lines correspond to the constant $\kappa$ values of $B_{c 1}$ and $B_{s h}$.

the Ginzburg-Landau equations, therefore I will not discuss the theoretical limiting field of layered surface in this dissertation.

The main advantage of the dirty layer technology is the possibility of merging together high accelerating gradients with high quality factors. Since the only condition to increase the gradient is a larger $\kappa_{s}$ at the surface, there are no limitations on how to generate it. A smart choice is to N-dope the first nanometers of the very surface - exactly what is achieved with the N-infusion treatment - in order to benefit also of the higher Q-factor.

In Figure 6.14, some sample Q-factor versus accelerating field curves are plotted. N-infused cavities (see Section 3.3) show larger accelerating gradient than the standard N-doped cavities and Q-factor two times smaller at medium field. Anyway, throughout the whole measurement the quality factor of N-infused cavities is about 


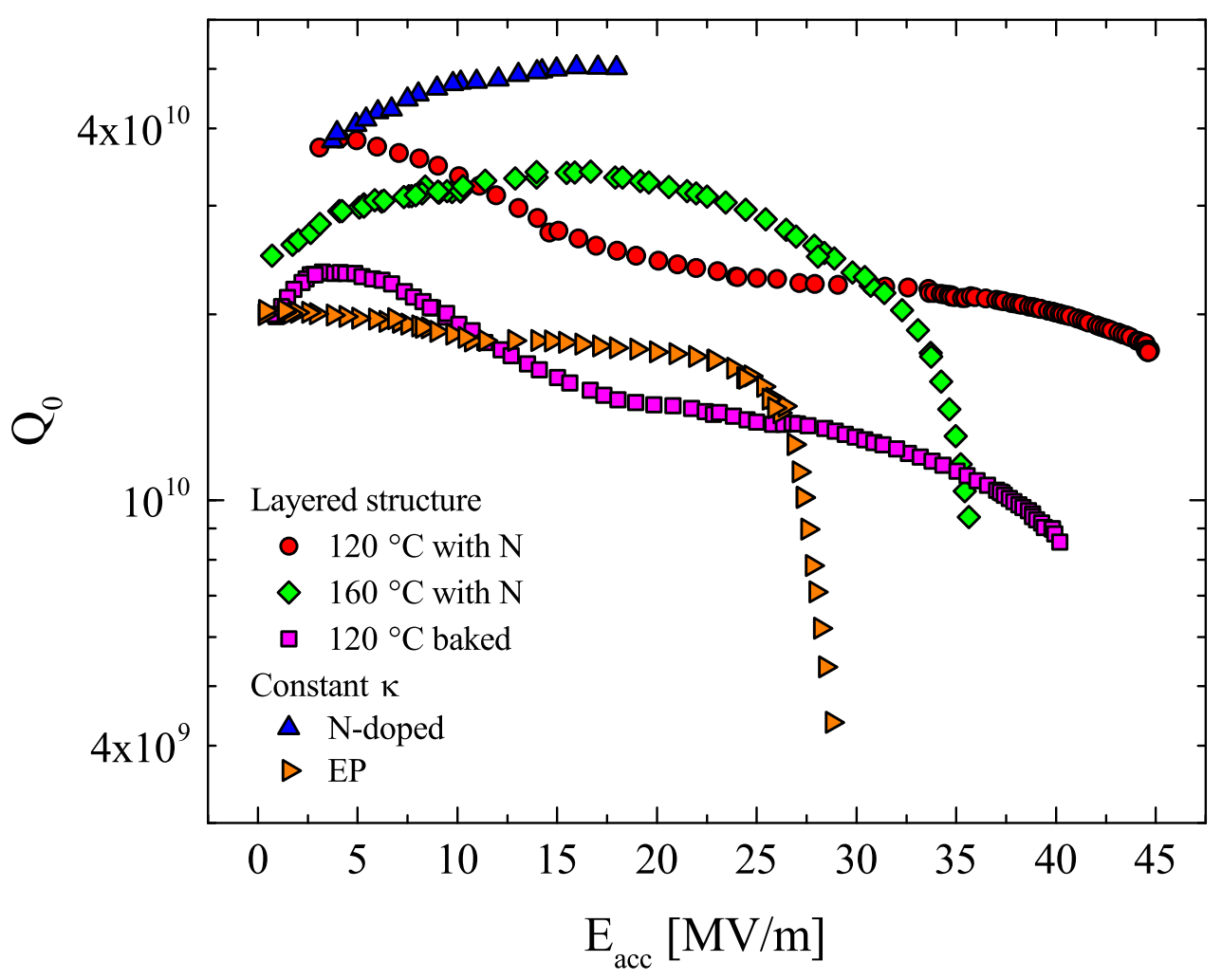

Figure 6.14. Quality factor versus accelerating field measured for representative cavities treated with different recipes.

2 two times larger than $120^{\circ} \mathrm{C}$ baked cavities. The largest Q-factor difference is at high fields $\left(E_{a c c}>35 \mathrm{MV} / \mathrm{m}\right)$ where $\mathrm{N}$-infused cavities have Q-factor two to three times larger than standard $120{ }^{\circ} \mathrm{C}$ baked cavities.

The larger Q-factor observed in N-infused cavities can be explained by assuming that the doped layer has lower surface resistance than the bulk. The total resistance is then generated by the parallel of the resistances of bulk and layer, weighted over the field profile penetrating in the superconductor. We should then expect that the larger the layer the higher the Q-factor. An example of such calculation is proposed in [113].

In Figure 6.14, the performance of two different N-infusion cavities are reported $\left(120{ }^{\circ} \mathrm{C}\right.$ and $\left.160{ }^{\circ} \mathrm{C}\right)$. As the temperature of the treatment is increased, the behavior 
of the cavity moves towards the one observed for N-doped cavities since the layer is thicker and it weights more on the final Q-factor shape.

\subsection{Summary}

In this chapter the theoretical description of the enhancement of the accelerating gradient of superconducting microwave cavities by means of a dirty layer grown at the cavity surface was theoretically described.

When a thin dirty layer with $\kappa$ greater than the bulk is grown at the very surface of the superconductor, it introduces only a perturbation on the magnetic induction profile at the surface, if its thickness is lower than the penetration depth of the field $\left(d<\lambda_{s}\right)$. On the other hand, the bulk properties are not affected by the presence of the layer at the surface, therefore the stability condition of vortices in the superconductor bulk are defined by the Ginzburg-Landau parameter of the bulk only.

In such scenario, the lower critical field of the whole structure is then defined by the bulk's $B_{c 1}$ and the lower limit of the field of first penetration range is enhanced, up to $B_{c 1}\left(\kappa_{b}\right)$. At the same time, the energy barrier to vortex nucleation is enhanced as well, and the vortex penetration may be delayed up to higher fields than the lower critical field of the bulk.

Such description of the accelerating gradient enhancement can explain why both $120{ }^{\circ} \mathrm{C}$ baked cavities and $\mathrm{N}$-infused cavities can quench statistically always above the lower critical field of their bulk. Indeed, both the two treatments were proven to produce a dirty layer at the surface, with thickness smaller than the penetration depth of the field. The standard Bean-Livingston barrier description is indeed not able to explain the experimental observations.

The technological advance promised by layered structures, such as the dirty layer on top of a clean bulk explained in this chapter, is of great impact. If the 
quality factor is doubled at high gradients, then the power dissipated by the cavity in the helium bath is two times less. In practical words, the cryogenic cost of the cavity operation can be decreased, especially when the accelerating gradient is large, introducing the possibility of decreasing the number of cryo-plants (or decreasing their power) needed to operate the accelerator at high fields. 


\section{CHAPTER 7 \\ QUALITY FACTOR DEGRADATION DUE TO QUENCH}

\subsection{Chapter Overview}

Generally, when accelerating cavities are quenched while operating their dissipation in increased, so that when the field is replenished in the resonator the quality factor is lower than before. The historical description of such phenomena defines that when the normal conducting region is created during the quench some magnetic flux can be trapped at the quench spot causing extra flux dissipation [119], decreasing the cavity quality factor.

The origin of such trapped magnetic flux remained unclear and was ascribed to different mechanisms, such as: thermal currents driven by the local thermal gradient in the quench zone [119], rf field trapped within the penetration depth region, or ambient magnetic field. However, the full understanding of the phenomenon has not been developed yet.

Previous studies $[120,121,122,123]$ of the quality factor degradation in high and medium $\beta$ superconducting resonators targeted a criterion for the amount of flux trapped during the quench. A clear dependence of the quench-related degradation on the locally applied non-uniform external magnetic field was found, highlighting the possibility that extra dissipation introduced by quenching was of environmental origin. The "quench annealing" phenomenon - recovery of the cavity quality factor by quenching when the additional field was removed-was also first documented in these studies.

In this chapter, the detailed physics behind the quality factor degradation due to quench in superconducting resonators is described [124]. The experimental evidence reported proves that the $Q_{0}$ degradation due to quench is a direct consequence 
Table 7.1. Cavities studied with respective thermal treatments and quench fields. Doped cavities were treated with 25 mTorr of $\mathrm{N}_{2}$ and with a post treatment chemistry (EP) of $5 \mu \mathrm{m}$.

\begin{tabular}{lcc}
\hline \hline Cavity & Processing treatment & Cavity type \\
\hline aes011 & $800{ }^{\circ} \mathrm{C}, 2$ min w N & +6 min w/o N \\
aes019 & $800{ }^{\circ} \mathrm{C}, 10$ min w N & Bare 1-cell \\
acc002 & $800{ }^{\circ} \mathrm{C}, 20$ min w N & Bare 1-cell \\
aes014 & $120{ }^{\circ} \mathrm{C}$ bake & Bare 1-cell \\
aes024 & $800{ }^{\circ} \mathrm{C}$, LCLS-II N-doping treatment [65] & Dressed 9-cell \\
\hline
\end{tabular}

of trapped ambient magnetic field, ruling out any other possible mechanisms. In addition, a dependence of the extra losses after quench on the orientation of the external magnetic field with respect to the cavity axis is found. The full recovery of $Q_{0}$ after quench can be achieved when the cavity is quenched in the absence of the external magnetic field - an alternative to warming up above the critical temperature - is described along with a consistent physical model of this phenomenon.

When the externally applied field is big enough ( $>1$ Oe), the trapped magnetic flux at the quench spot might migrate far from the quench spot, and the complete recovery of the quality factor is not possible anymore.

\subsection{Experimental Set-up}

Several quench experiments were performed using multiple $1.3 \mathrm{GHz}$ fine grain bulk Nb cavities of elliptical TESLA shape [15]. Three bare 1-cell cavities and one LCLS-II dressed 9-cells were prepared by nitrogen doping recipes, and one 1-cell was prepared by standard $120^{\circ} \mathrm{C}$ baking (see Table 7.1 ). N-doped cavities (9-cell included) were baked for 3 hours at $800{ }^{\circ} \mathrm{C}$ before the doping treatment. All measurements were done at the Fermilab vertical test facility. A more detailed explanation of the doping treatment and of the $120{ }^{\circ} \mathrm{C}$ bake are reported in Section 3.3. Schematics of the 


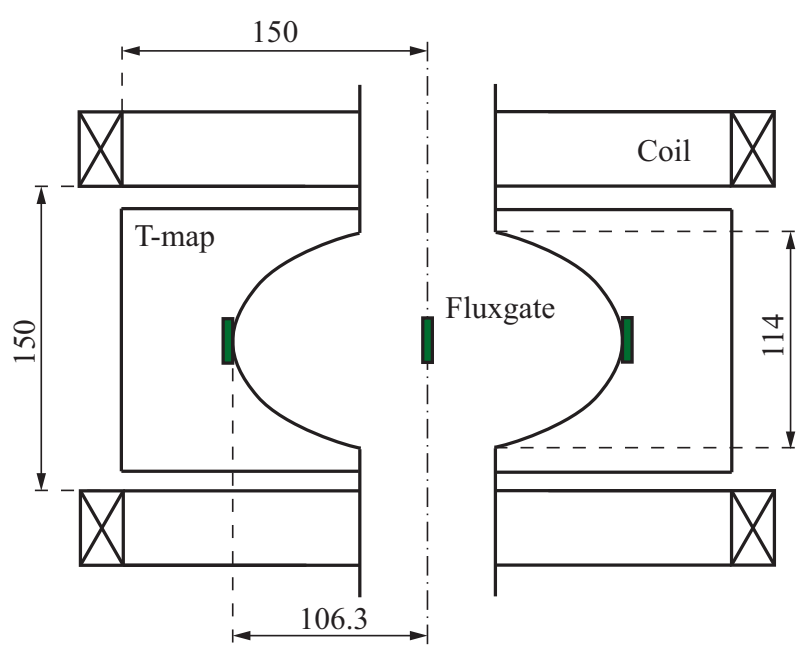

a)

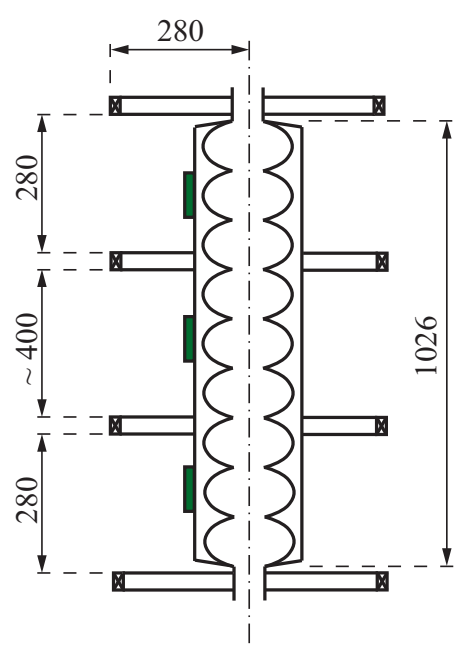

b)

Figure 7.1. Experimental setup for: (a) 1-cell cavities, (b) 9-cell fully dressed LCLS-II cavity. All the dimensions are given in millimeters [124].

cavity instrumentation used are presented in Figure 7.1.

In order to map the temperature variation over the cavity wall during quench, localize the quench spot site, and study in detail the resulting dissipation pattern, 1-cell cavities were equipped with the T-map system. The external magnetic field was sustained by Helmholtz coils and measured by four single-axis Bartington Mag- $01 \mathrm{H}$ cryogenic fluxgate magnetometers positioned at the equator axially to the cavity and spaced about $90^{\circ}$ between each other-see Figure 7.1a for the schematic. For one of the cavities (aes011), two sets of Helmholtz coils were used generating fields in two different directions (axial and orthogonal). In this configuration no temperature mapping was used due to space constraints.

As shown in Figure 7.1b, the fully dressed LCLS-II 9-cells cavity (aes024) was equipped with two sets of Helmholtz coils and three fluxgate magnetometers placed outside of the helium vessel.

In order to minimize the temperature dependent part of the surface resistance 
(see Section 2.5), all the measurements except for the 9-cells cavity (measured only at $2 \mathrm{~K}$ ) were done at the lowest temperature achievable by the cryo-plant, which was around $1.5 \mathrm{~K}$.

\subsection{Experimental Results}

All the measurements were performed by quenching cavities in the presence of the external magnetic field $(H)$ or in compensated magnetic field, and by recording the degradation of $Q_{0}$ caused by the quench at a fixed accelerating field. The quench events considered are caused only by "hard" limiting factors (e.g. thermal breakdown), whereas multipacting- or field emission-related quenches were not considered in this study. The very low compensated magnetic field $(<1 \mathrm{mG})$ was achieved by adjusting the Helmholtz coils current in order to cancel out the magnetic field naturally present in the vertical measurement cryostat $(\lesssim 5 \mathrm{mG})$.

7.3.1 Quenching in Compensated Ambient Fields. The first series of quenches were performed in compensated external magnetic fields. All quenches were "hard", reached by increasing the rf field. As Figure 7.2 clearly shows, no appreciable $Q_{0}$ degradation was observed after quenching tens of times in compensated field (red star) meaning that no extra dissipation was introduced for all the investigated bare cavities, even though they were prepared with different treatments. The same lack of degradation was also the case for the fully dressed 9-cells cavity treated with the LCLS-II nitrogen doping recipe, for which the average magnetic field value achieved by compensation coils just before the quench was lower than 2 mOe.

This phenomenon is important as it rules out all other possible mechanisms of magnetic flux generation and trapping during quench except for static ambient field, as those would necessarily lead to a decrease in $Q_{0}$ even in zero ambient field. In other words, magnetic flux trapped at the quench spot is not generated intrinsically 


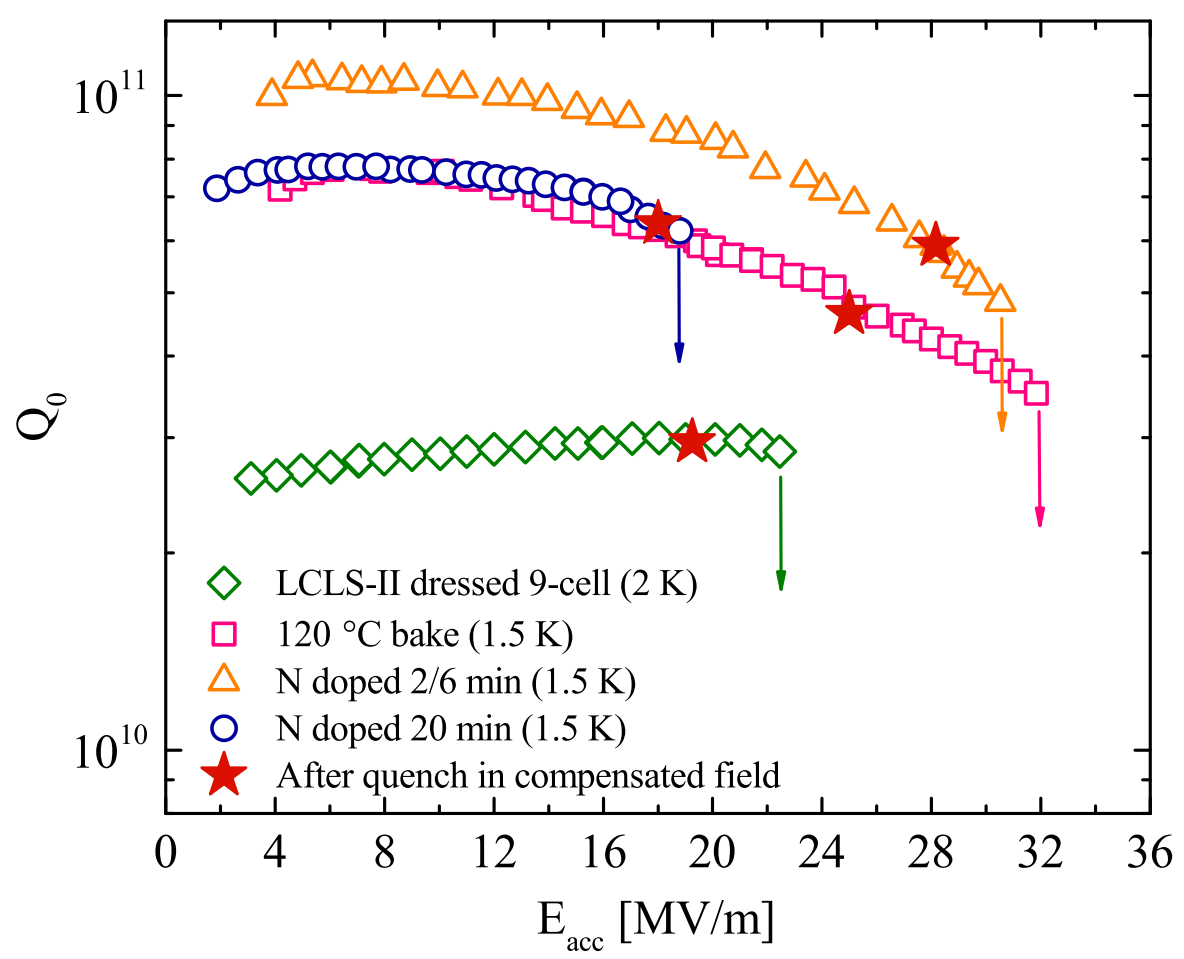

Figure 7.2. $Q_{0}$ versus accelerating field curves acquired after a cool-down in compensated field before any quench. The red stars correspond to the $Q_{0}$ point acquired after quenching $>10$ times in compensated external magnetic field [124].

but the quench.

\subsubsection{Degradation in Non-Compensated Ambient Magnetic Field and Re-}

covery by Zero-Field Quenching. In the second series of experiments a finite value of the magnetic field was applied outside of the cavity before quenching. The degradation of the Q-factor was clearly observed after a single and a number of quenches, as shown in Figure 7.3a, where $\Delta R_{0}(H)$ corresponds to the difference in averaged $R_{0}=270 \Omega / Q_{0}$ after and before any quenches. Then, in each case the ambient field was again adjusted to as low as possible, and the cavity was quenched again several times (points with "0" field labels in Figure 7.3a). $Q_{0}$ could be totally recovered to its value just before any quenches, as it was also observed in [120].

Interestingly, multiple quenches in the same field are needed for the residual 


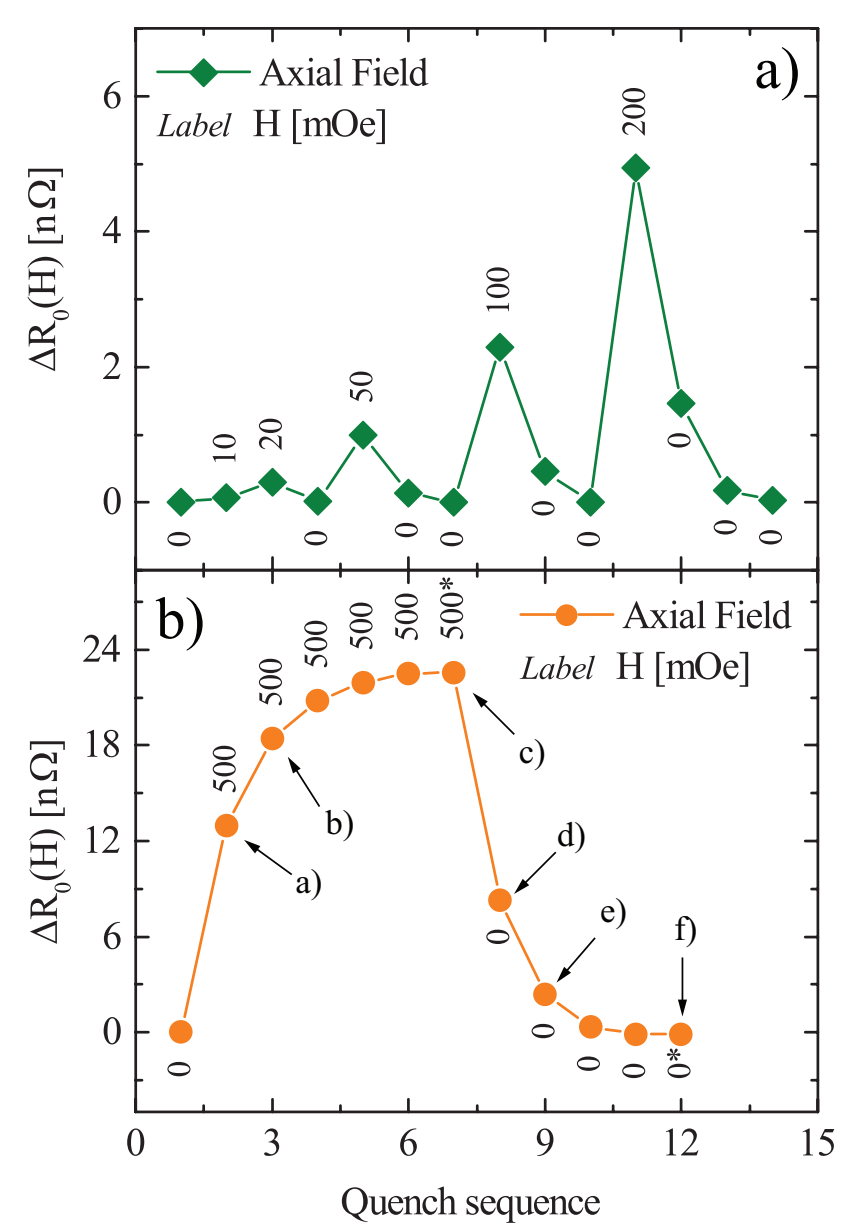

Figure 7.3. Quench study performed on cavity acc002: (a) variation of the residual resistance due to quenches in the presence of external magnetic field; (b) saturation of the residual resistance due to multiple quenches in the same external field. The labels "0" indicate the condition of compensated field, while the symbol "** refers to multiple quenches. $\Delta R_{0}(H)$ points that correspond to the T-maps of Figure 7.4 are indicated with arrows and letters [124].

resistance to reach a higher saturation value, as can be seen in Figure 7.3b. Such a saturation suggests that the maximum possible value of the magnetic flux trapped at the quench spot for a specific external magnetic field level was reached. Correspondingly, $Q_{0}$ could be totally recovered by several (and not a single) quenches in the compensated low field.

It is possible to gain a detailed insight into what happens during the multiquench saturation and recovery of $Q_{0}$ by analyzing the corresponding T-maps. In 


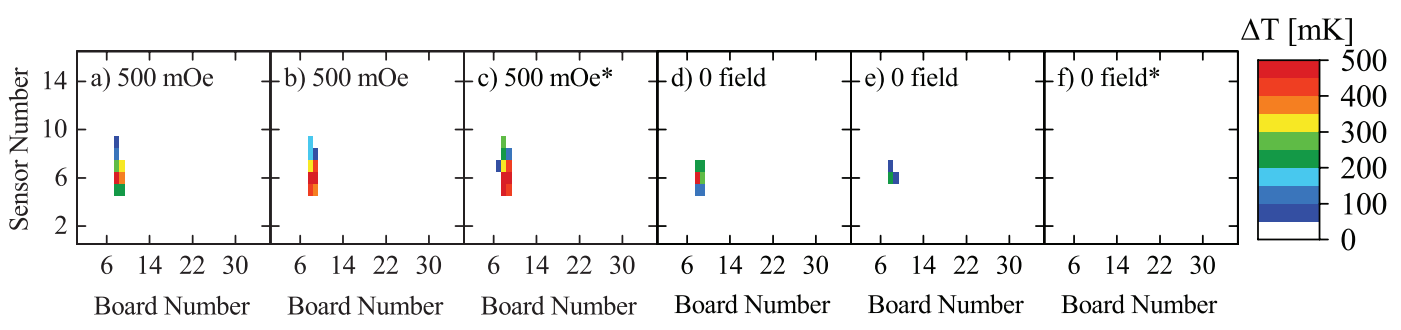

Figure 7.4. Evolution of the dissipation due to trapped field at the quench spot for acc002 after quenching: (a) a single, (b) two, (c) multiple times in 500 mOe, and after quenching (d) a single (e) two, (f) multiple times in compensated field. The symbol "** identifies multiple quenches. All the T-maps were acquired at $E_{a c c}=18 \mathrm{MV} / \mathrm{m}[124]$.

Figure 7.4, a sequence of temperature maps corresponding to the evolution of the magnetic flux trapped at the quench spot are shown. The corresponding residual resistance changes are highlighted with arrows in Figure 7.3b.

As it can be clearly observed, each of the quenches in 500 mOe (Figure 7.4a-c) leads to the progressive increase of the dissipation around the quench spot until the saturation is reached. Subsequent quenches in zero field (Figure 7.4d-f) cause the gradual decrease of the local dissipation until the pre-quench extra dissipation-free situation is attained, indicating the annihilation of the trapped flux.

The same $Q_{0}$ recovery effect was observed for all the cavities tested (Table 7.1), independently on their surface preparation.

7.3.3 Effect of the Magnetic Field Orientation. The effect of the external field orientation on the $Q_{0}$ degradation during quench was studied on one of the cavities (aes011) in a series of single quenches in the presence of either non-zero axial or nonzero orthogonal components of the external field $H$, with respect to the cavity beam axis. In Figure 7.5, $\Delta R_{0}(H)$ is plotted as a function of the applied field. For the same magnetic field level $\Delta R_{0}(H)$ is always higher for the orthogonal component, implying that most likely a larger amount of field was trapped. 


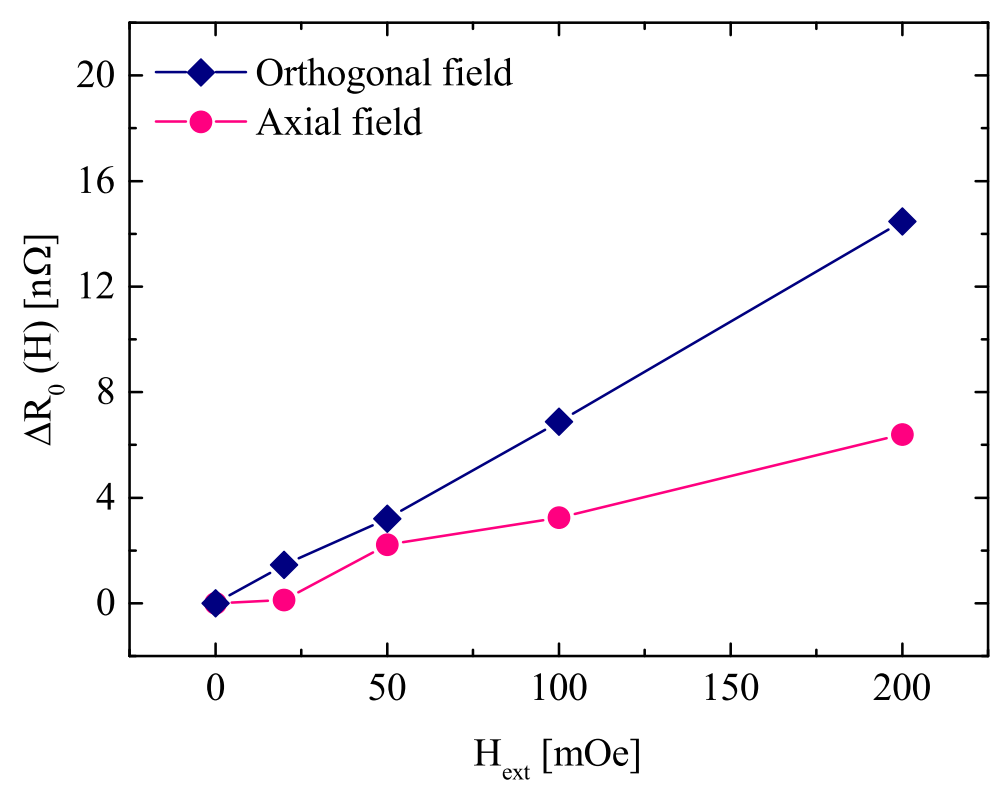

Figure 7.5. Variation of the residual resistance of aes011 after single quenches for different values and orientations of the external magnetic field [124].

In the Meissner state the superconducting phase behaves as a perfect diamagnet, and the magnetic field is expelled from the cavity bulk and confined outside leading to the redistribution of the local field amplitudes at the cavity surface. In Figure 7.6, a COMSOL Multiphysics simulation of the cavity in the Meissner state is shown for different field directions. As it is clearly seen, the final field configuration is strongly dependent upon the cavity axis orientation with respect to the applied field, and for a given axis orientiation the local field amplitude is strongly position-dependent on the cavity surface as well.

The local field amplitude at the quench spot might be different than that measured by the fluxgate at the equator. Furthermore, for the same quench spot and for the same magnitude of the applied field, by varying the field orientation with respect to the cavity axis, the local magnetic field value at the quench spot changes (as shown in Figure 7.6). Therefore, different $Q_{0}$ degradation for different magnetic field components may be then just a manifestation of this purely geometrical effect. 

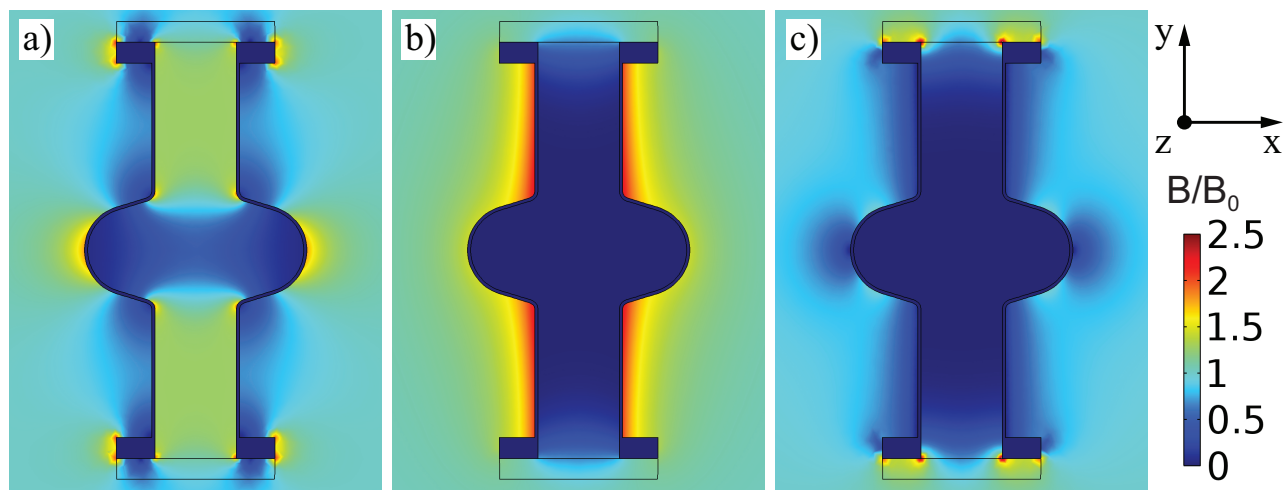

Figure 7.6. Perfect Meissner effect simulation for different orientation of the magnetic field. Figure (a) field applied along y, (b) along z and (c) along x direction [124].

7.3.4 Irrecoverable $Q_{0}$ Degradation. The experiments were extended to higher values of magnetic field ( $\gtrsim 1 \mathrm{Oe}$ ). Once the cavity is quenched multiple times and the residual resistance is saturated, the cavity quality factor can only be partially recovered compared to its original value before quench. This phenomenon is demonstrated in Figure 7.7 for aes019, where the residual resistance could be recovered to its original value only when the quench was performed in magnetic field values less or equal to $700 \mathrm{mOe}$.

After the cavity was quenched several times in 1 Oe, its quality factor could not be completely recovered anymore, even by quenching several times in compensated field. The same behavior was also observed for cavities acc002 and aes011, for which the magnetic field threshold above which the quality factor could not be completely recovered was $700 \mathrm{mOe}$ and $300 \mathrm{mOe}$ respectively. Cavity aes014 was quenched in fields up to 700 mOe, but no irrecoverable $Q_{0}$ threshold was observed.

In Figure 7.8, the evolution of the local dissipation due to trapped field at the quench spot for the recoverable and irrecoverable $Q_{0}$ degradation of aes019, as registered by the T-map system, is shown. The corresponding residual resistance variation is shown in Figure 7.7. Figure 7.8a reveals the dissipation due to trapped magnetic flux after quenched several times in 700 mOe. The ambient field was then 


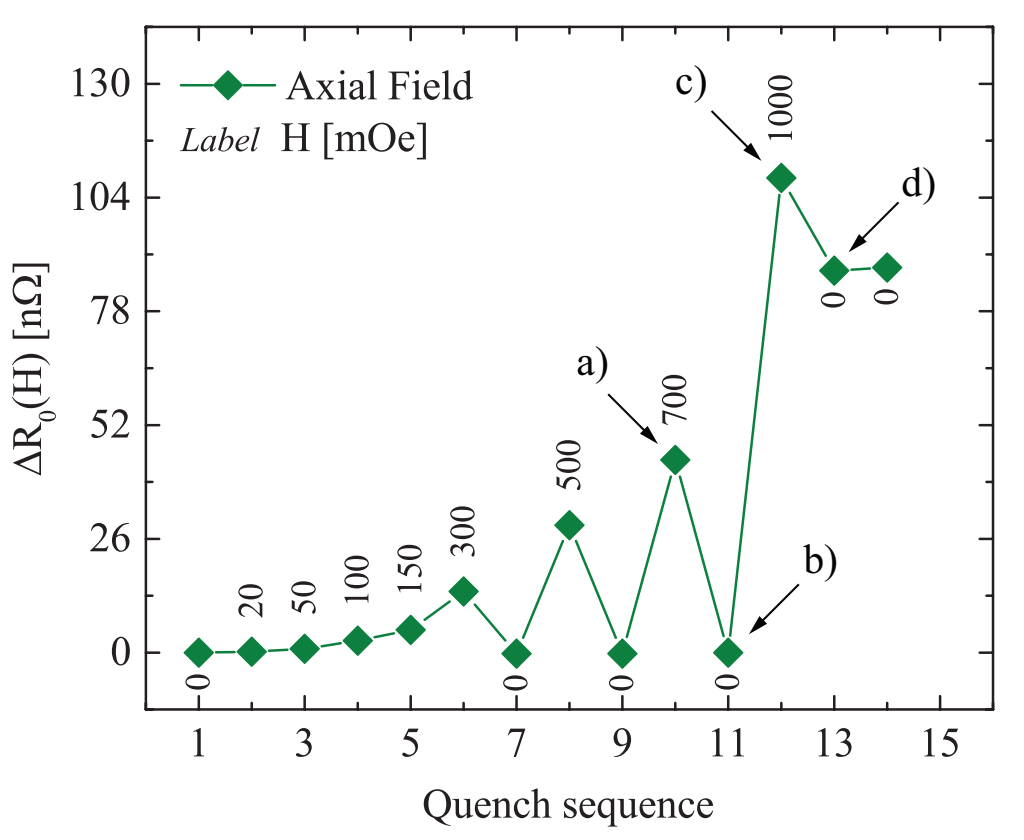

Figure 7.7. Residual resistance evolution of aes019 after quenching in different field values. Every point in the graph correspond to multiple quenches in the same applied field. The arrows indicates the data points that correspond to the T-maps of Figure 7.8. The labels "0" indicate the condition of compensated field [124].

compensated as much as possible, and the cavity was again quenched several times. Figure 7.8b indicates that most of the dissipation introduced by the previous quenches vanished. Still, some flux remained trapped at the quench spot, probably because of the non-axial field components of the field in the cryostat that could not be compensated by the axial coils.

The external magnetic field was then set to 1 Oe and the cavity quenched several times again. The corresponding T-map in Figure $7.8 \mathrm{c}$ shows that the vortex dissipation area has now spread out further than after 700 mOe quenches. In addition, two dissipative "lobes" clearly emerged separated by a less-dissipative region in the middle. After the field was subsequently compensated and the cavity quenched several times no complete field annihilation occurred, while some redistribution of the magnetic flux was recorded, as shown in Figure 7.8d.

\subsection{Magnetic Field Redistribution During Quench}




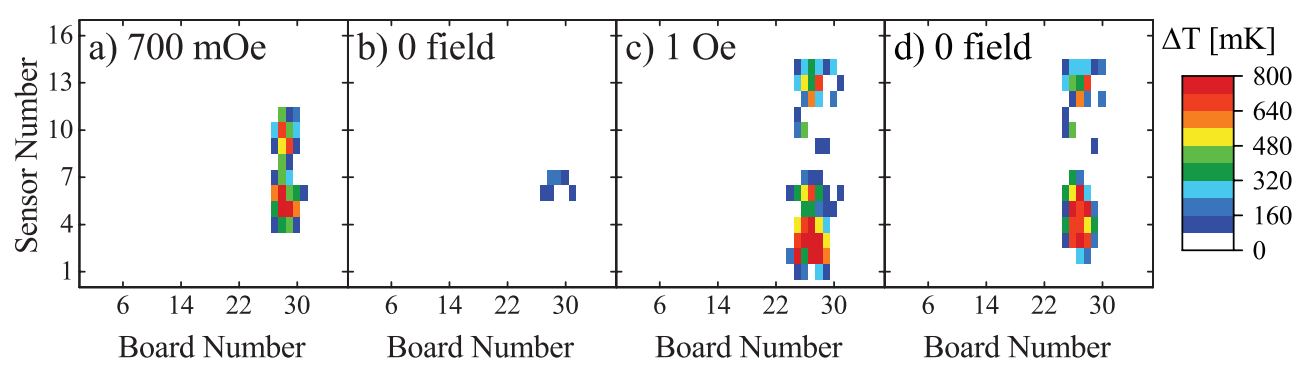

Figure 7.8. T-map images acquired after the cavity aes019 was quenched in presence of external magnetic field with the following sequence of magnitudes: (a) $700 \mathrm{mOe}$, (b) zero field, (c) 1 Oe and (d) zero field. Such sequence shows the impossibility of $Q_{0}$ recovery after the cavity was quenched in 1 Oe. The most part of the trapped flux could not be annihilated quenching again in zero field. All the T-maps were acquired at $E_{a c c}=17 \mathrm{MV} / \mathrm{m}[124]$.

In order to interpret the experimental results, it is first important to understand the dynamics of the magnetic flux during the quench event. In order to visualize this, COMSOL Multiphysics was used to perform magnetic field simulations during a quench event.

In the Meissner state before quench, the magnetic field (axial case is shown) is expelled from cavity walls and deflected around it, as shown in Figure 7.9a. During the cavity quench, a normal conducting hole opens on the cavity wall, which causes the redistribution of the magnetic induction $B$. These changes can be described as driven by the magnetic force, which is given (per unit volume) by

$$
\mathcal{F}_{\mathbf{m}}=-\nabla\left(\frac{B^{2}}{2 \mu_{0}}\right)+\frac{(\nabla \cdot \mathbf{B}) \mathbf{B}}{\mu_{0}},
$$

where the first term corresponds to the magnetic pressure, and the second one to the magnetic tension. The magnetic pressure is directed perpendicular to the magnetic field lines in the opposite direction to the field gradient. The magnetic tension is instead only present when the magnetic field is bent, and it has radial direction with aim directed toward the center of curvature. It introduces the same restoring action that the elastic force has when a stiff slab is bent. The magnetic tension then exerts a force to straighten out the bent magnetic field line. 

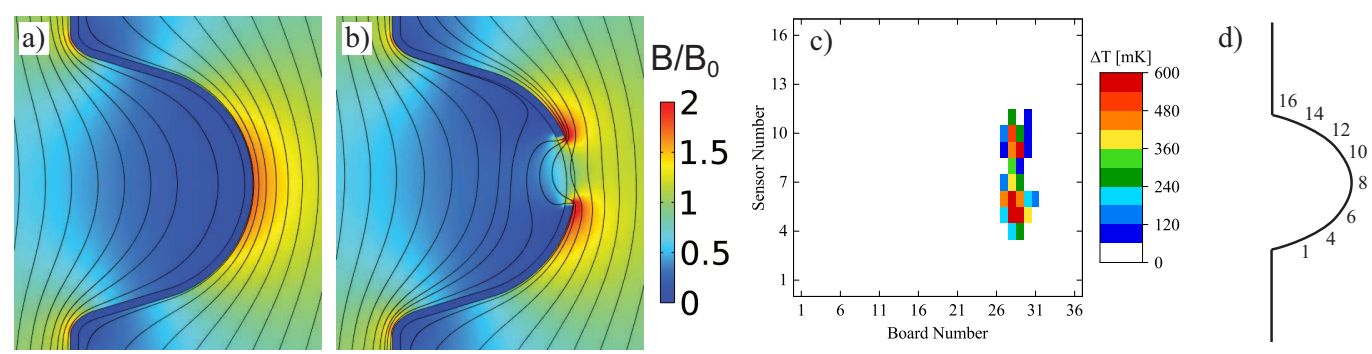

Figure 7.9. Simulations of the magnetic field distribution around the quench spot: (a) before quench; (b) during quench. Color scale represents the ratio between the local magnetic field and the applied magnetic field. (c) T-map of aes019 after multiple quenches in $500 \mathrm{mOe}$ (acquired at $E_{a c c}=17 \mathrm{MV} / \mathrm{m}$ ); (d) schematics of the thermometer positions [124].

Around the equatorial zone of the cavity the magnetic field lines are denser and more bent by the cavity in the Meissner state (Figure 7.9a), thus both the magnetic pressure and the magnetic tension are directed towards the cavity wall. When the normal conducting hole opens on the cavity wall, the magnetic field that was excluded from the cavity internal volume is now allowed to penetrate driven by the sum of the magnetic pressure and magnetic tension contributions.

In order to simulate the field configuration once trapped at the quench spot, the normal-superconducting boundary was simulated by using the following approximate sigmoidal form of the field-dependent relative magnetic permeability $\mu_{r}(H)$ :

$$
\mu_{r}(H)=\frac{1}{1+e^{-(H-\alpha) / c}}
$$

where $\alpha=c \cdot \ln (0.0001)+H_{c 2}$ is the parameter to ensure $\mu_{r} \cong 1$ for $H \geq H_{c 2}$, $H_{c 2}=H_{c 2}(0)\left[1-\left(T / T_{c}\right)^{2}\right], H_{c 2}(0)=410 \mathrm{mT}$ is the upper critical field at $0 \mathrm{~K}$, $T_{c}=9.25 \mathrm{~K}$ is the critical temperature, and $c$ is the parameter that defines the slope of $\mu_{r}(H)$ through transition, the smaller $c$ the steeper the function.

To approximate best the sequential progression of the field redistribution during quench, a point-like heat source was assumed in the equatorial zone (but not right at the equator), where quench most frequently occurs, and let the local temperature 
$T$ linearly increase with time. As the temperature increases, the local critical field $H_{c 2}(T)$ of niobium decreases, and when the external applied field $H$ exceeds $H_{c 2}(T)$ (so that $\mu_{r} \cong 1$ ), the external field starts penetrating the cavity wall at the heated region. Parameters used for niobium are thermal conductivity $\kappa=30 \mathrm{Wm}^{-1} \mathrm{~K}^{-1}$, the heat capacity $\mathrm{C}_{p}=0.126 \mathrm{Jkg}^{-1} \mathrm{~K}^{-1}$ and the thermal boundary resistance at the niobium-liquid helium interface $\mathrm{h}_{k}=5000 \mathrm{Wm}^{-2} \mathrm{~K}^{-1}$.

Simulated field distribution for the moment in time when the normal zone opening is largest is shown in Figure $7.9 \mathrm{~b}$. It can be seen that the magnetic field lines form a semi-loop inside the cavity volume, and when the quench region is cooled again below $T_{c}$, the field will be trapped in the form of vortices with magnetic field in the opposite direction - entering on one side of the normal zone and exiting from another.

In Figure 7.9c, a T-map taken during rf measurements of cavity aes019 after a series of quenches in $500 \mathrm{mOe}$ is shown, which is consistent with two lobes of dissipation corresponding to entry and exit points of the field lines in accordance with the simulation (Figure 7.9d can be used as a reference to understand the T-map image orientation and temperature sensor locations).

\subsection{Quality Factor Recovery Mechanism}

The suspected mechanism at the basis of the $Q_{0}$ recovery phenomenon is the annihilation of the magnetic field trapped at the quench spot when the cavity is allowed to quench again but in very low compensated field [125, 126].

With a finite magnitude of the applied magnetic field, the trapped magnetic field lines will create a closed loop passing through the two Helmholtz coils (Figure 7.10a). But when the external field is canceled out, in order to respect the Ampere's law the trapped magnetic field must be sustained only by the screening 
a)

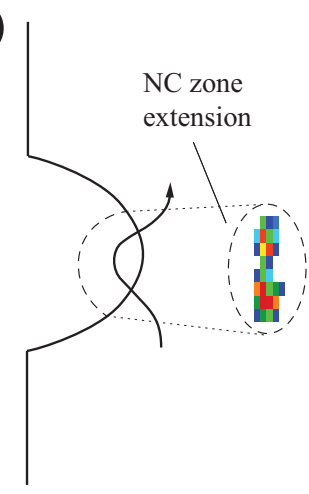

b)

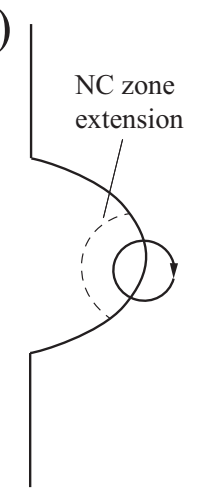

c)

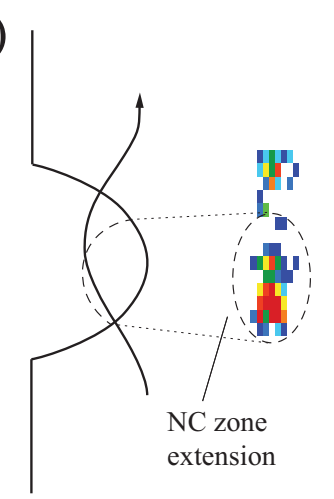

d)

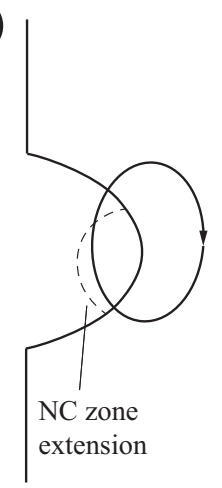

Figure 7.10. Sketch of the magnetic field trapped at the quench spot: (a) after quench in the presence of external magnetic field - the T-map shows a two-lobe shaped dissipation pattern; (b) after the external field cancellation; (c) trapped magnetic flux after the flux migration - the T-map shows two hot spots; (d) field compensated after the magnetic flux migration [124].

currents in the superconductor (Figure 7.10b).

When the quench occurs, a normal conducting region is created at the quench spot, and the trapped field vanishes upon the superconducting/normal transition annihilating the superconducting screening currents that sustained it. The dimension of the emerging normal-conducting region is governed by the total dissipated energy, which is set by the value of the maximum accelerating field at which the cavity quenches. Thus, if the quench field remains the same, the normal opening size should also be the same, and all the trapped field within the quench zone can be annihilated.

In this simple model, the trapped magnetic flux can be annihilated only if: i) it is trapped in the loop configuration, and ii) if vortex entry and exit points are both inside the maximum extension of the normal conducting area during the quench.

If the condition ii) is not satisfied (e.g. Figure 7.10c) and some of the vortex entry/exit points fall outside of the maximum extension of the normal conducting zone during the quench, then even if the external field is compensated and the cavity quenched again, those superconducting currents that sustain the trapped field outside 
of the normal opening will still exist, preventing the full annihilation of the trapped magnetic flux. Such a situation may occur either if the trapped flux is migrated away from the original location (discussed in detail below), or if the quench field is decreased due to the extra dissipation introduced by the trapped flux. The normal conducting opening size would be then smaller than before and less field can be annihilated.

As it was shown in Subsection 7.3.2, more than one quench is needed in order to reach a saturation level of $\Delta R_{0}(H)$ when quenching in non-zero field, and several quenches in zero field are typically needed to completely recover the quality factor, the more so the higher the trapped field. These findings can be interpreted as the possible manifestation of the finite time constant $\tau_{B}$ for the magnetic field configuration to change during the normal zone opening. The value of $\tau_{B}$ is determined by the damping time of eddy currents, which counteract the penetration of the magnetic field [127]. If the characteristic time of the normal zone existence $\tau_{n c} \sim 100 \mathrm{~ms}[128]$ is shorter than $\tau_{B}$, then the magnetic field distribution in the quench area will only reach the steady state after a number of quenches $N \sim \tau_{B} / \tau_{n c}$.

Lastly, it is important to emphasize again that the quality factor recovery in compensated field reveals the extrinsic nature of the magnetic flux trapped during quench. If the magnetic flux was intrinsic to the cavity, or in other words generated by the quench itself (i.e. local thermal currents), then the recovery of $Q_{0}$ could be achieved only by warming the cavity above $T_{c}$, as quenching in zero applied magnetic field would only be a source of extra dissipation as any other quench.

\subsection{Magnetic Flux Migration}

The equation of motion of a single vortex in the absence of drift current can be described by assuming thermal force, viscous drag force, Magnus force and pinning force, described in Section 2.4. In the present case though, vortices and anti-vortices 
are mutually connected sharing the same magnetic field lines. It means that the motion of the two connected vortices is coupled via the magnetic tension. The main action of such a force is to straighten the magnetic field lines that connect vortices and anti-vortices, pulling them apart. The modified equation of motion is then:

$$
\frac{\Sigma}{N} \frac{(\nabla \cdot \mathbf{B}) \mathbf{B}}{\mu_{0}}-S \cdot \nabla T-\eta \dot{\mathbf{x}}-\gamma n_{s} e\left(\dot{\mathbf{x}} \times \phi_{0} \hat{n}\right)-\mathbf{F}_{\mathbf{p}}=0
$$

The first term corresponds to the magnetic tension per vortex with $\mathbf{B}$ - the trapped field, $\Sigma$-the normal surface area through which the magnetic field bent inside the cavity volume passes and $N$ - the number of vortices. The second term is the thermal force, whose direction depends only on the thermal gradient $\nabla T$, and which pushes a vortex toward colder regions (as described in Section 2.4). The origin of the temperature gradient is the increased local rf dissipation at the trapped flux location, which makes the thermal force directed to spread the trapped flux around.

Here the trapped fields is considered far below $H_{c 2}$, and therefore the interaction between different vortices, which is a rapidly decreasing function of the inter-vortex spacing [33], is neglected. The time-dependent Lorentz force acting between the surface screening currents in the cavity and the trapped flux is neglected as well, since its net effect is the oscillation of the surface segments of the magnetic flux around the stable equilibrium position. Such force is needed in order to define the dissipation introduced by the trapped vortices (as described in Chapter 4), but not to describe the collective migration of the vortices observed.

The combination of the magnetic tension and thermal force will act against the Magnus force $\gamma n_{s} e\left(\dot{\mathbf{x}} \times \phi_{0} \hat{n}\right)$, the viscous drag force $\eta \dot{\mathbf{x}}$, and the pinning force $\mathbf{F}_{\mathbf{p}}$. Here $n_{s}$ is the superelectron density, $\gamma$ is the fraction of the Magnus force that is active, and the vortex motion viscosity per unit length $\eta$ is given by [44]:

$$
\eta=\frac{3}{2} \frac{\sigma_{n} \phi_{0}^{2}}{\pi^{2} \xi_{0} l}
$$


with $\sigma_{n}$ - the normal electron conductivity, $\xi_{0}$ - the coherence length, and $l$ - the electron mean free path.

Vortices starts migrating when the sum of magnetic tension and thermal force becomes larger than the pinning force:

$$
\frac{\Sigma}{N} \frac{(\nabla \cdot \mathbf{B}) \mathbf{B}}{\mu_{0}}-S \cdot \nabla T_{k} \geq \mathbf{F}_{\mathbf{p}} .
$$

Magnetic tension in Eq. 7.3 plays a crucial role as it allows to explain why the motion of lobes happens along a straight line. If the thermal force was the only driving force of flux migration then the net motion would be isotropic, i.e. we would see the lobes becoming broader and more blurry as the local temperature is increased driven by the rf dissipation. What we see instead is the lobes moving in the opposite directions along the same line, as it is expected if the magnetic tension were also nonnegligible. Both contributions are dependent on the amount of magnetic field trapped during the quench. The higher the trapped magnetic field, the larger the local thermal gradient appearing when the rf field is reestablished inside the resonator, and therefore the larger the thermal force. Similarly, the magnetic tension term is proportional to $B^{2}$ and is higher for larger trapped fields.

Using the flux motion description, we can estimate the pinning potential strength that would correspond to the observed vortex migration thresholds. We approximate the pinning potential as an ideal inverse Lorentzian curve [84] that is acting on the whole flux line crossing the cavity wall.

$$
U_{p}(x)=-\frac{U_{0}}{1+\left[\left(x-x_{0}\right) / \xi\right]^{2}},
$$

where $x_{0}$ is the initial vortex position, $U_{0}$ is the pinning potential depth, and $\xi=$ $\left(1 / l+1 / \xi_{0}\right)^{-1}$ is the effective coherence length that determines the depth of the pinning potential well. 

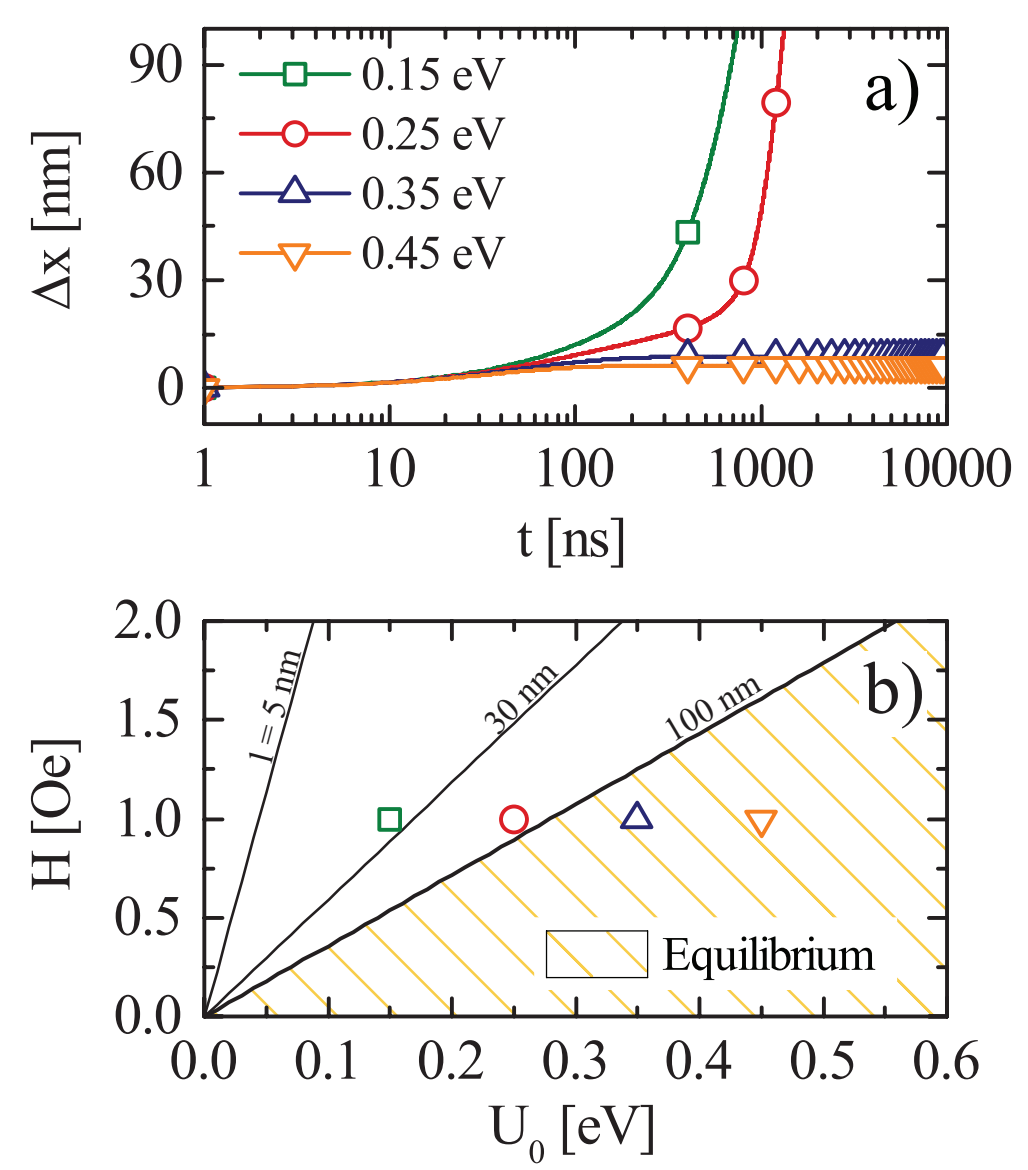

Figure 7.11. The numerical solutions of the vortex motion equation (Eq. 7.3) for the displacement $\Delta x$ as a function of time for different pinning potentials is plotted in (a). The magnetic field considered in the calculation was 1 Oe, with mean free path $100 \mathrm{~nm}$. The separatrix line between equilibrium and vortex migration is plotted in (b) (see Eq. 7.5). Different lines correspond to different mean free paths. The points refer to magnetic field $B$ and potential $U_{0}$ chosen for the numerical solutions in (a) [124].

The equation of motion (Eq. 7.3) is then numerically solved with the program Wolfram Mathematica, considering the flux line length equal to the cavity wall thickness and small displacements near the pinning potential minimum, which allows taking the radius of curvature for the magnetic field lines constant. The number of vortices $N$ was estimated as equal to the lobe area $A$ times the trapped magnetic field $B$, over the flux quantum: $N=A \cdot B / \phi_{0}$. Fixed parameters for the simulation are trapped field $B=1$ Oe, thermal gradient $|\Delta T| \approx 1.7 \mathrm{~K} \cdot \mathrm{cm}^{-1}$ and the flux area $A=4 \pi \mathrm{cm}^{2}$ (both estimated from the T-map in Figure $7.8 \mathrm{~d}$ ), the coherence length 
$\xi_{0}=39 \mathrm{~nm}$ [85], and the mean free path $l=100 \mathrm{~nm}$. Simulation starts from $t=0$ when the normal conducting zone has just finished closing, and trapped flux starts moving driven by thermal and magnetic forces.

Figure 7.11a shows the simulation results for the vortex displacement $\Delta x$ as a function of time for different values of the pinning potential $U_{0}$. In the case where $U_{0}$ is such that Eq. 7.5 is not satisfied, the vortex displacement approaches an equilibrium value of $\Delta x \approx 5 \div 10 \mathrm{~nm}$ from the potential minimum after $\sim 1 \mu \mathrm{s}$.

When instead Eq. 7.5 is respected, the vortex motion deviates from the previous situation. Initially the vortex drifts experiencing the pinning force, but after a certain time - depinning time - it depins and its displacement from the pinning site grows drastically (as shown in Figure 7.11a for $U_{0}=0.15 \mathrm{eV}$ and $0.25 \mathrm{eV}$ ). It is important to notice that deeper pinning potential wells correspond to longer depinning times.

Figure $7.11 \mathrm{~b}$ shows the separatrix line between the regions of constrained pinned and unconstrained depinning (Eq. 7.5) motion of flux, for pairs of trapped magnetic field $B$ and pinning potential $U_{0}$ values, and different mean free path values (labels on the lines). For the points from Figure 7.11a the separatrix falls in between the points for $U_{0}=0.25 \mathrm{eV}$ and $0.35 \mathrm{eV}$, giving the threshold value of $U_{0} \simeq 0.28 \mathrm{eV}$, which is a reasonable value for pure metals [84].

This simple migration model can also explain why different thresholds were observed. Assuming that different cavities might have different pinning potentials, we expect that the magnetic field threshold will be affected from that - the higher the pinning potential the higher the magnetic field threshold for the migration phenomenon.

An insightful analysis is shown in Figure 7.12 for the redistribution of the 

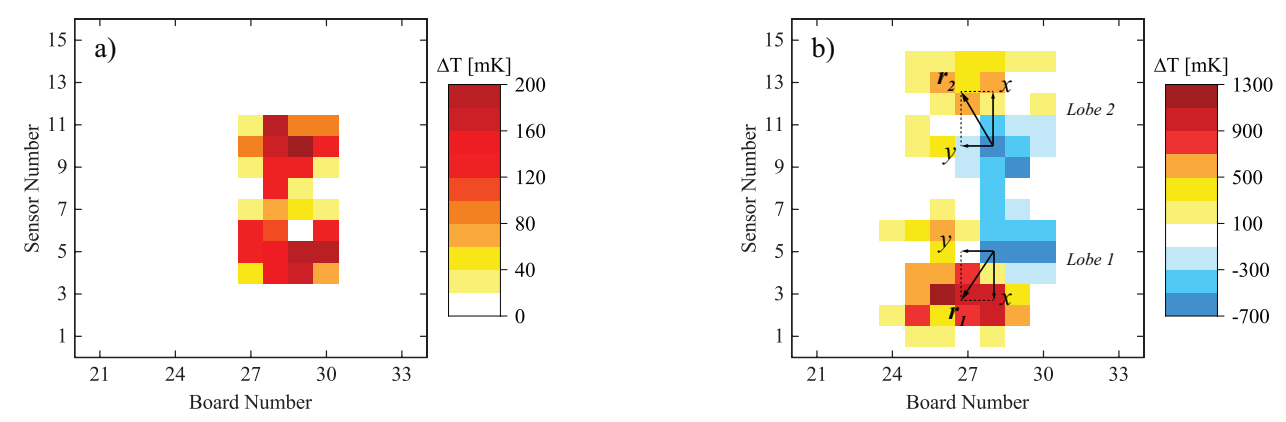

Figure 7.12. Redistribution of the magnetic flux. In figure (a) the difference between the T-map acquired after quenching in $700 \mathrm{mOe}$ and in $500 \mathrm{mOe}$ is reported, while in (b) between quenching in 1 Oe and in 700 mOe. The flux redistributes/adds from negative to positive values regions [124].

trapped flux for different quenches for cavity aes019. The relative changes were obtained by subtracting T-maps acquired after quenches in different external field values. Negative $\Delta T$ values in Figure 7.12 reveal zones from which the flux moved away, positive $\Delta T$ zones indicate the areas where the flux was added, and $\Delta T=0$ corresponds to areas where the flux remained the same. In Figure 7.12a such an incremental difference is shown for the trapped flux dissipation after quenching in 700 mOe as compared to quenching in 500 mOe. Clearly, no field redistribution occurred, and simply more flux was trapped. This case is consistent with the analysis above for the trapped field that is not high enough and vortices are displaced by only tens of nanometers from their initial post-quench position, therefore no migration is observed with the T-map.

The pattern is clearly different in Figure $7.12 \mathrm{~b}$, which shows the difference after quenching in $1000 \mathrm{mOe}$ with respect to $700 \mathrm{mOe}$. In this case a clear redistribution of the trapped magnetic field was found. The flux trapped after quenching in $1000 \mathrm{mOe}$ migrated away from the central zone: Lobe 1 followed the path indicated by the unit vector $\mathbf{r}_{\mathbf{1}}$, while Lobe 2 followed along the direction of unit vector $\mathbf{r}_{\mathbf{2}}$. Such a situation can emerge when Eq. 7.5 is satisfied and the pinning force is overwhelmed by magnetic tension and thermal forces, and consequently two lobes are pushed further apart. 
Both $\mathbf{r}_{1}$ and $\mathbf{r}_{2}$ vectors can be decomposed into their components $x$ and $y$, and surprisingly both possess a non-zero component along $y$ (see Figure 7.12b). As $y$-component is orthogonal to the path dictated only by the magnetic tension - along $x$-it should be attributable to some force orthogonal to the vortex motion, Magnus force being the likely suspect. The direction of the orthogonal motion is the same for the two lobes, which is also compatible with Magnus force acting on outgoing flux from Lobe 1, and incoming flux from Lobe 2 (see Section 2.4). For extremely pure superconductors Magnus force was shown to play an important role [33, 41], and high purity $\mathrm{Nb}(\mathrm{RRR} \sim 300)$ out of which cavities are made for our studies may also fall in this class.

\subsection{Summary}

In this chapter the description of the dissipation introduced by the quench event was discussed. When the cavity is quenched, the magnetic flux trapped at the quench spot is of pure extrinsic origin - ambient magnetic field.

The key supporting finding is no extra dissipation $\left(Q_{0}\right.$ is not affected) introduced by quenches in zero magnetic field, allowing every intrinsic mechanism of flux trapping - i.e. generation of thermal currents or trapping of rf field-to be ruled out. Such conclusion is further corroborated by: i) the clear relation of dissipation introduced by quenching to the orientation of the applied magnetic field, and ii) the possibility to totally recover the quality factor - without warming the cavity above $T_{c}$ - by compensating the external field and re-quenching several times. Interestingly, for larger values of the ambient field (that allows magnetic and thermal forces larger

than the pinning force) the Q-factor recovery may become impossible due to the outward flux migration beyond the normal zone opening during quench.

The flux migration process is attributed to the synergistic action of the thermal 
force generated by thermal gradients caused by the rf dissipation of the trapped flux itself, and the magnetic tension on the trapped flux lobes against the pinning force. If one of the two lobes migrates outside of the maximum extension of the normal conducting region during the quench, the $Q_{0}$ recovery becomes not possible. A transverse component in the vortices migration path was also observed, which is compatible with the presence of a non-negligible fraction of Magnus force.

Different magnetic field thresholds for the migration were observed. Based on simulations, this phenomenon is most likely introduced by different values of pinning potential that affects the migration - the deeper the pinning potential, the larger the minimum value of trapped magnetic field needed to ignite the flux migration. 


\section{CHAPTER 8}

\section{CONCLUSIONS}

The scope of this dissertation was to improve the phenomenological understating of some fundamental limitations of SRF cavities. As discussed in Section 1.2, by an accurate phenomenological description we may be able to overcome the current limitations of SRF cavities, and in principle allow for more affordable large superconducting accelerators.

\subsection{Quality Factor Limitations due to Pinned Vortices}

The limitations to the quality factor, as described in Section 2.5, are associated to the finite value of surface resistance. In particular, in Chapter 4 the resistance associated to trapped magnetic vortices in the resonator was discussed.

The surface resistance due to trapped vortices is described exhaustively in terms of the interplay of two different regimes: the pinning and the flux flow regime respectively. Experimental data acquired for $1.3 \mathrm{GHz}$ SRF cavities show a peculiar bell-shape trend as a function of the electron mean free path, i.e. the purity of the material. The vortex surface resistance increases as a function of the mean free path in the pinning regimes, for low mean free path values. In the large mean free path region instead (flux flow regime), the vortex resistance decreases.

Such opposite trends of the vortex surface resistance in these two mean free path limits, can be explained by imaging that the vortex motion is counteracted by the pinning force alone for small $l$, and from the viscous drag force alone for large $l$. In the pinning regime the real part of the vortex resistivity can be approximated as $\rho_{1} \approx \omega^{2} \eta / p^{2}$, and in the flux flow regime as $\rho_{1} \approx 1 / \eta^{2}$. While $\eta$ increases with the mean free path, $p$ decreases with it, and such opposite behavior generates the ball-shaped trend observed. 
In the pinning regime the motion equation can be written neglecting the viscous drag force, since way smaller than the pinning force. The motion equation so defined describes a total reactive response of vortices, and therefore the surface resistance tends to zero. On the other hand, for large $l$ the motion equation may be written neglecting the pinning force instead, returning a pure resistive behavior of the vortex dissipation. The vortex response may then be thought as varying from total reactive in the small mean free path limit to total resistive in the large mean free path limit.

Important to notice that only in the pinning regime the resistance has a dependence on the frequency, hence we should expect that in the flux flow regime all the cavities behaves in the same way, independently on the frequency. As the frequency is increased the vortex resistance peak is then enhanced since in the pinning regime $\rho_{1} \propto \omega^{2}$.

By means of this simple description of the vortex surface resistance we can predict that for small frequencies the dissipation is lowered and the peak of the surface resistance suppressed. Such finding can have an important technological followup: low frequency cavities may be screened less efficiently from the magnetic field than high frequency one, and therefore cut the cost of the magnetic shielding.

Moreover, by introducing artificial strong pinning defects in the cavity (as is done for superconducting magnets) the vortex surface resistance should in principle decrease driven by a stronger pinning force, and therefore limit the dissipation due to trapped flux. If we assume that such defects are not increasing the intrinsic residual resistance, but are only changing the pinning strength, then the Q-factor per some amount of trapped field should increase.

\subsection{Accelerating Gradient Limitation and Enhancement}


The fundamental limitation of the maximum achievable gradient is found to be dependent on the very surface condition of the cavity, as reported in Chapter 5.

Statistically, N-doped cavities are observed to quench below $B_{c 1}$, so far none of them was seen reaching the meta-stable Meissner state. T-map measurements suggest that $\mathrm{N}$-doped cavities are limited by the penetration of magnetic field at the quench spot. All the N-doped cavities tested with the T-map did not present any preheating at the location where the quench was going to happen. Also, since most of such cavities are usually seen to quench below $B_{c 1}$, the quench ignition mechanism is suspected to be magnetic field enhancement.

Differently, $120{ }^{\circ} \mathrm{C}$ baked cavities are statistically seen to quench in the metastable Meissner state, well above the lower critical field. Such finding is of particular interest since from the energetic of vortex penetration we would expect such cavities to quench at lower fields than N-doped.

In Chapter 6 the Bean-Livingston barrier to the vortex penetration was calculated for constant Ginzburg-Landau parameter $\kappa$. The calculation shows that as $\kappa$ is increased, the energy barrier keeping vortices out of the superconductor when $B>B_{c 1}$ is lowered. For such a reason we would expect $120^{\circ} \mathrm{C}$ baked cavities to quench at $B_{c 1}$ as the N-doped. In $120{ }^{\circ} \mathrm{C}$ baked cavities, the vortex penetration is instead delayed, since their very surface present a thin dirty layer that delays the vortex penetration.

A thin dirty layer grown at the very surface of the superconductor, with $\kappa$ greater than the bulk and thickness lower than the penetration depth at the surface $\left(d<\lambda_{s}\right)$, introduces only a perturbation on the superficial magnetic induction profile. On the other hand, the bulk properties are not effect by the presence of the layer at the surface, therefore the condition of stability of vortices in the superconductor 
bulk are not affected by the dirty layer at the surface, and are defined by the bulk Ginzburg-Landau parameter only.

Even if the surface is dirtier than the bulk, the lower critical field of the whole structure is still defined by the bulk's $B_{c 1}$. At the same time, the energy barrier to vortex nucleation is enhanced as well, and the vortex penetration may be delayed up to higher fields than the lower critical field of the bulk. In this scenario then, the vortex nucleation and therefore the cavity quench are enhanced. The lower limit of possible quench is then set by the lower critical field of the bulk, and not by the surface condition. This means that if N-doped cavities are prepared in such a way to present same $\kappa$ at the very surface, but clean bulk, their lower field limit for vortex penetration can be enhanced up to the lower critical field of the bulk $H_{c 1}\left(\kappa_{b}\right)$.

The superficial dirty layer can be produced in different ways. A smart choice is to N-dope only few nanometers $(10-20 \mathrm{~nm})$ of the $\mathrm{rf}$ surface. In such a way the benefices of both dirty layer and N-doping are exploited, and cavities can be pushed up to higher fields with higher Q-factors. Such behavior was indeed observed in Ninfused cavities, which possess a thin dirty doped layer at the surface. With such new treatment Q-factors of about $2 \cdot 10^{10}$ at $45 \mathrm{MV} / \mathrm{m}$ are possible.

Looking forward to the future construction of the ILC, this new treatment is extremely important. Because of the 2 times larger Q-factor at high fields than standard high gradient $\left(120^{\circ} \mathrm{C}\right.$ baked) resonators, cavities can be operated at the same gradient with half of the dissipated power. More than that, the lower dissipation at high gradients allows the accelerating field specification for the ILC $(31.5 \mathrm{MV} / \mathrm{m})$ to be raised to higher values, implying a huge cost reduction in terms of civil engineering, cavity and cryomodule production, as discussed in Section 1.2.

\subsection{Quality Factor Degradation by Quench}


Quench in accelerating cavities may in general increase the dissipation once the electromagnetic field in reestablished in the resonator. In Chapter 7 the purely extrinsic origin of the magnetic flux trapped at the quench spot due to quench is proven. The possible alternative mechanisms of magnetic flux generation during the quench, such as thermal currents or trapping of rf field are ruled out. Indeed, no extra dissipation $\left(Q_{0}\right.$ is not affected) is introduced by quenches in a magnetic free environment.

The study highlighted further effects that are proof of the total extrinsic origin of the flux trapped at the quench spot. Firstly, the extra dissipation introduced by the quench is related to the orientation of the applied magnetic field with respect the cavity. Secondly, once the quality factor is degraded after a quench, the total recovery to its initial valued is achievable by re-quenching several times in a magnetic field-free environment (compensating the external applied field to values as low as possible). If the magnetic flux generation at the quench spot was even only partially of intrinsic nature, then by re-quenching in compensated field the dissipation should increase, and certainly not decrease as instead observed.

The complete recovery of the quality factor by re-quenching in compensated field is achievable only up to a certain value of external magnetic field. For larger ambient field values the Q-factor recovery may become impossible due to the outward flux migration beyond the normal zone opening during quench.

Such flux migration process is generated by the combined effect of thermal force and magnetic tension acting against the pinning force. The position of the two dissipation lobes observed with the T-map system, is crucial to explain the recovery mechanism. If one of the two lobes migrates outside of the maximum extension of the normal conducting region during the quench, the $Q_{0}$ recovery becomes not possible. Based on simulations, the values of trapped magnetic field and pinning potential 
define the condition of migration. If the field trapped is large and the pinning potential shallow, then the migration is more like to happen, and the recovery impossible.

In SRF applications, these results can be helpful for $Q_{0}$ preservation in accelerators utilizing cavities at very high $Q_{0}$ values (requiring very challenging magnetic field shielding and cooldown process), as well as for designing cryomodules where SRF structures need to operate nearby sources of high magnetic field (usually solenoids or quadrupole magnets). 
APPENDIX A DIMENSIONLESS GINZBURG-LANDAU EQUATIONS 
In order to be independent on the material parameters (except for the GinzburgLandau parameter $\kappa$ ), the Ginzburg-Landau equations defined in Eq. 2.12, can be expressed in dimensionless quantities.

Let's consider the first Ginzburg-Landau equation in Eq. 2.12. If we normalize the order parameter $\Psi$ with respect the order parameter that minimizes the free energy $\Psi_{0}$ (Eq. 2.10), we can rewrite the equation as:

$$
f-|f|^{2} f+\frac{1}{2 m^{*} \alpha}\left(\frac{\hbar}{i} \nabla-e^{*} \mathbf{A}\right)^{2} f=0,
$$

with $f=\Psi / \Psi_{0}$, and by introducing the quantity $\xi=\hbar / \sqrt{2 m^{*} \alpha}$, the coherence length, it becomes:

$$
f-|f|^{2} f+\xi^{2}\left(\frac{\nabla}{i}-\frac{e^{*}}{\hbar} \mathbf{A}\right)^{2} f=0 .
$$

We can then normalize the quantities $x, y, z$ and $\mathbf{A}$ as defined in [103]:

$$
\begin{aligned}
& \left\{\begin{array}{l}
x \rightarrow \frac{x}{\lambda} \\
y \rightarrow \frac{y}{\lambda} \Rightarrow \nabla \rightarrow \frac{1}{\lambda} \nabla \\
z \rightarrow \frac{z}{\lambda}
\end{array}\right. \\
& \mathbf{A}=\mathbf{a} \sqrt{2} B_{c} \lambda
\end{aligned}
$$

where $\lambda$ is the penetration depth of the magnetic field and $B_{c}$ the thermodynamic critical field. Substituting such quantities in Eq. A.2, we get:

$$
f-|f|^{2} f+\frac{\xi^{2}}{\lambda^{2}}\left(\frac{\nabla}{i}-\frac{\sqrt{2} e^{*} B_{c} \lambda^{2}}{\hbar} \mathbf{a}\right)^{2} f=0 .
$$

At this point using the definition of Ginzburg-Landau parameter $\kappa$ [103]:

$$
\kappa=\frac{\lambda}{\xi}=\frac{\sqrt{2} e^{*} B_{c} \lambda^{2}}{\hbar},
$$

we obtain the first dimensionless Ginzburg-Landau equation:

$$
f-|f|^{2} f-\left(\mathbf{a}+\frac{i \nabla}{\kappa}\right)^{2} f=0 .
$$


Let's now consider instead the second Ginzburg-Landau equation in Eq. 2.12. We apply the same normalization of the order parameter, and by meaning of the Maxwell equations we express the current density as $\mathbf{J}=1 /\left(\mu_{0}\right) \nabla \times(\nabla \times \mathbf{A})$. The second Ginzburg-Landau equation takes then the form:

$$
\frac{\sqrt{2} H_{c}}{\left|\Psi_{0}\right|^{2} \lambda} \nabla \times(\nabla \times \mathbf{a})-\frac{e^{*} \hbar}{i 2 m^{*} \lambda}\left(f^{*} \nabla f-f \nabla f^{*}\right)+\frac{\sqrt{2} e^{* 2} B_{c} \lambda}{m^{*}}|f|^{2} \mathbf{a}=0 .
$$

Using the Ginzburg-Landau parameter definition (Eq. A.5) and $\left|\Psi_{0}\right|^{2}=m^{*} /\left(\mu_{0} e^{* 2} \lambda^{2}\right)[25]$ we obtain the second dimensionless Ginzburg-Landau equation:

$$
\nabla \times(\nabla \times \mathbf{a})+\frac{i}{2 \kappa}\left(f^{*} \nabla f-f \nabla f^{*}\right)+|f|^{2} \mathbf{a}=0
$$




\section{APPENDIX B}

VORTEX SURFACE RESISTANCE NUMERICAL CODE 


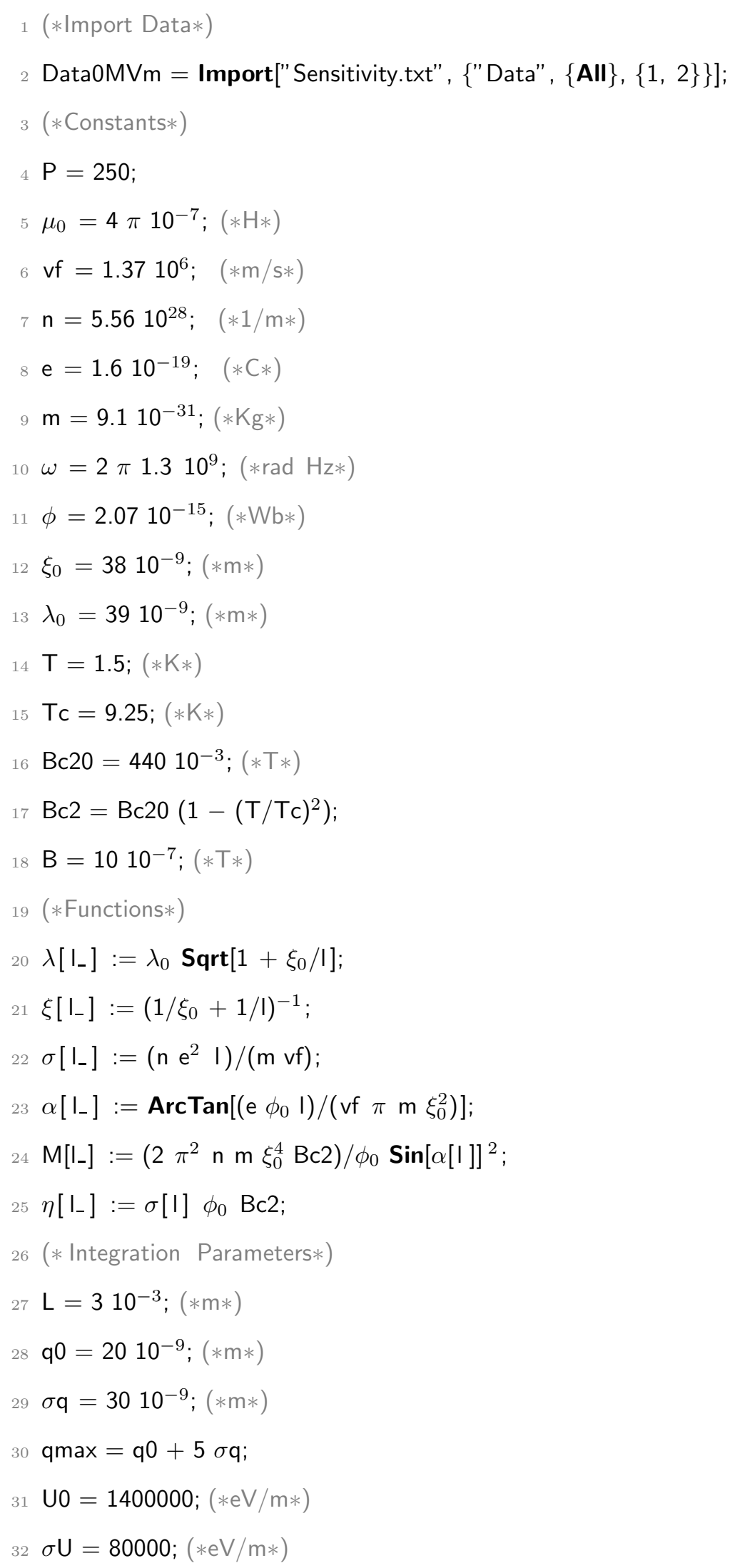




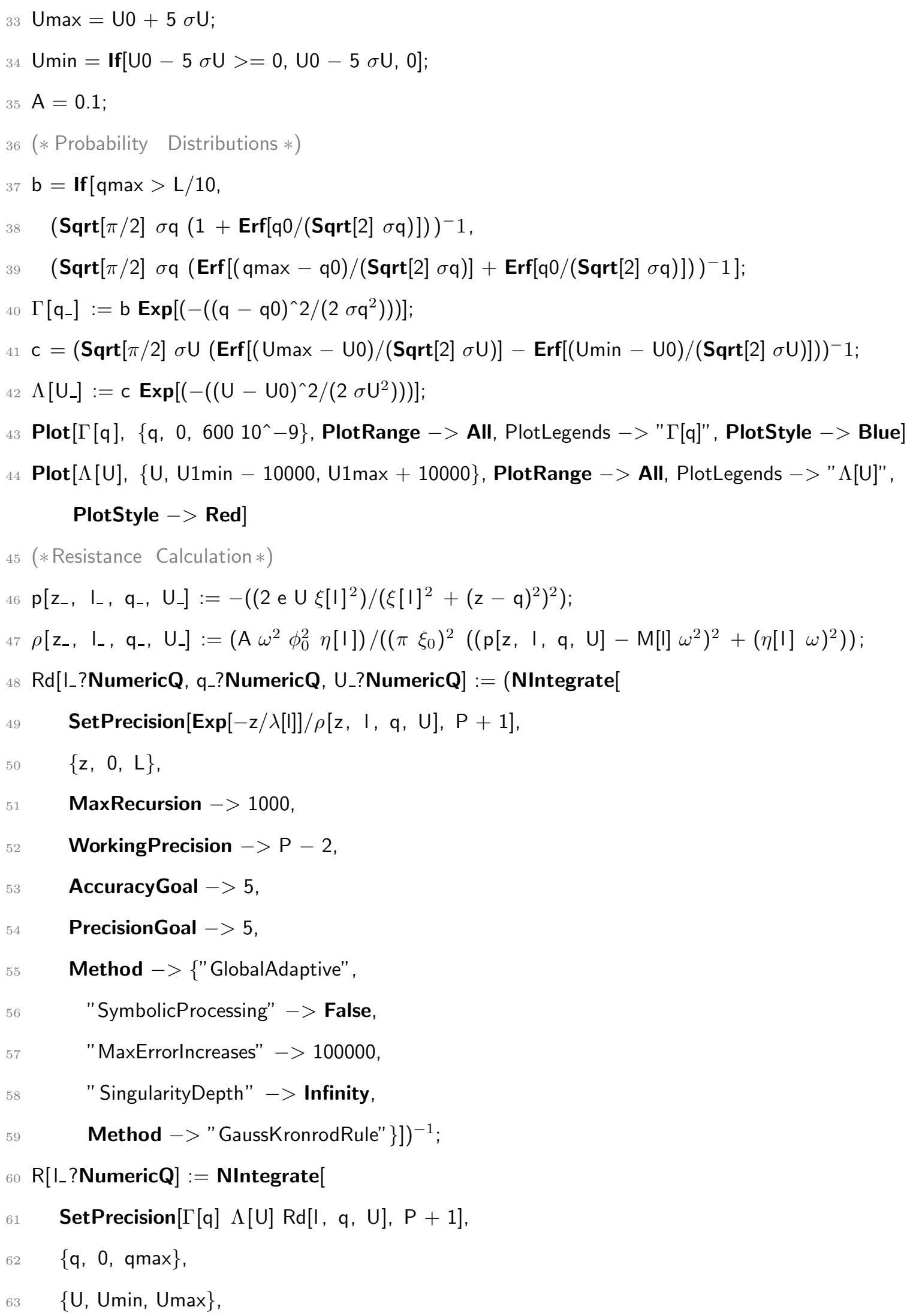




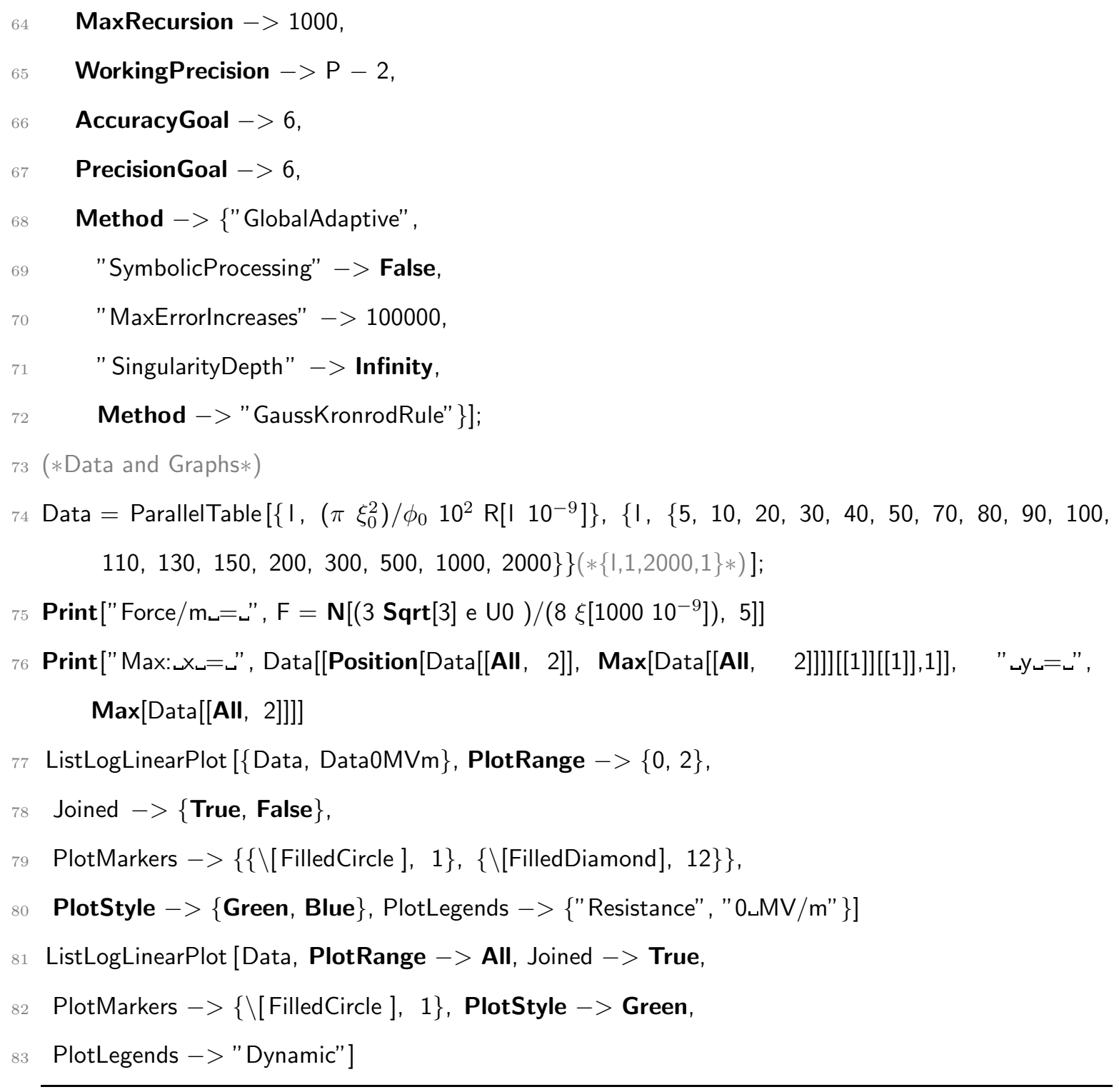


APPENDIX C SHOOTING METHOD 
The shooting method is a technique to solve numerical boundary value problems (BVP) by reducing them to initial value problems (IVP). The method is based on the fact that IVPs are usually less complicated to solve than BVPs. Therefore, a particular BVP that may not even converge to solution can be solved correctly and in a acceptable amount of time, by implementing the correct correspondent IVP.

The main idea is to transform the boundary value problem in a correspondent initial value problem, "shooting" the solution from the starting side of the resolution domain by means of an initial value solver, and "aiming" to the other end in such a way to match the boundary conditions. Such procedure is repeated until the solution converges to its correct form. In other words, the initial value problems is adjusted till its solution is also solution of the BVP.

Let us consider a boundary value problem of a second order ordinary differential equation:

$$
y^{\prime \prime}(x)=f\left(x, y(x), y^{\prime}(x)\right)
$$

with boundary conditions:

$$
\begin{aligned}
& y\left(x_{0}\right)=y_{0} \\
& y(X)=Y .
\end{aligned}
$$

Such BVP can be transformed in its representative IVP introducing the following initial conditions:

$$
\begin{aligned}
& y\left(x_{0}\right)=y_{0} \\
& y^{\prime}\left(x_{0}\right)=q,
\end{aligned}
$$

where $q$ must be chosen in such a way that the solution satisfies the boundary condition $y(X)=Y$. In order to choose the right initial value $q$, we can define a function $F(q)=y(X ; q)-Y$ which correspond to the difference of the IVP solution at $X$ (when the initial condition imposed is $y^{\prime}\left(x_{0}\right)=q$ ) and the boundary value $Y$. In 
other words, $F(q)$ represent how much the IVP solution at the boundary position $X$ is far from the BVP condition at the same point, as a function of the initial condition $q$.

If we now calculate the root of the function $F(q)$, we find the value $q$ for which $F(q)=0$, which corresponds to the correct initial value needed by the IVP to generate a solution that is also solution of the BPV. 


\section{APPENDIX D}

\section{$H_{C 1}$ AND $H_{S H}$ NUMERICAL CODES}




\section{D.1 Lower Critical Field Numerical Code}

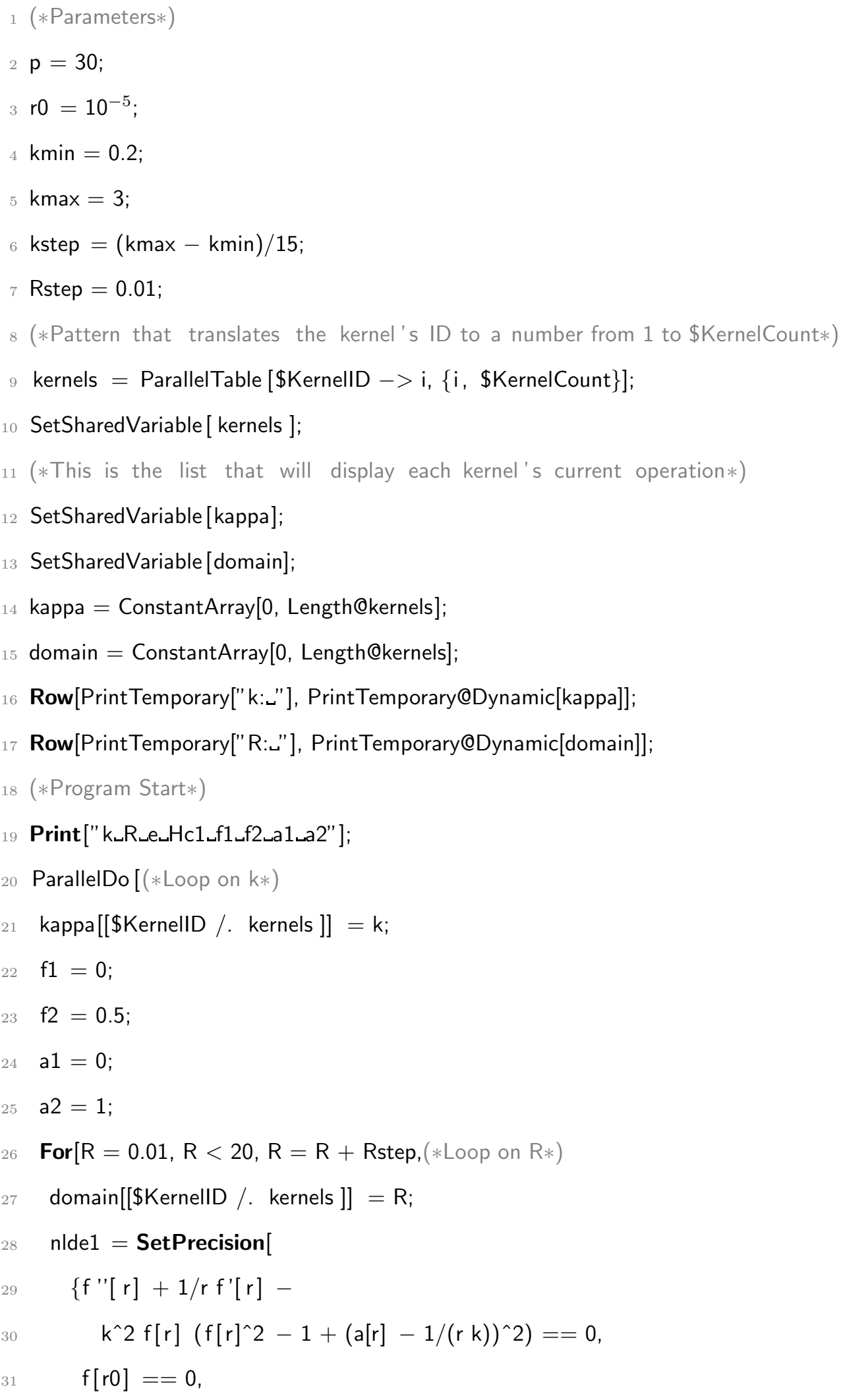




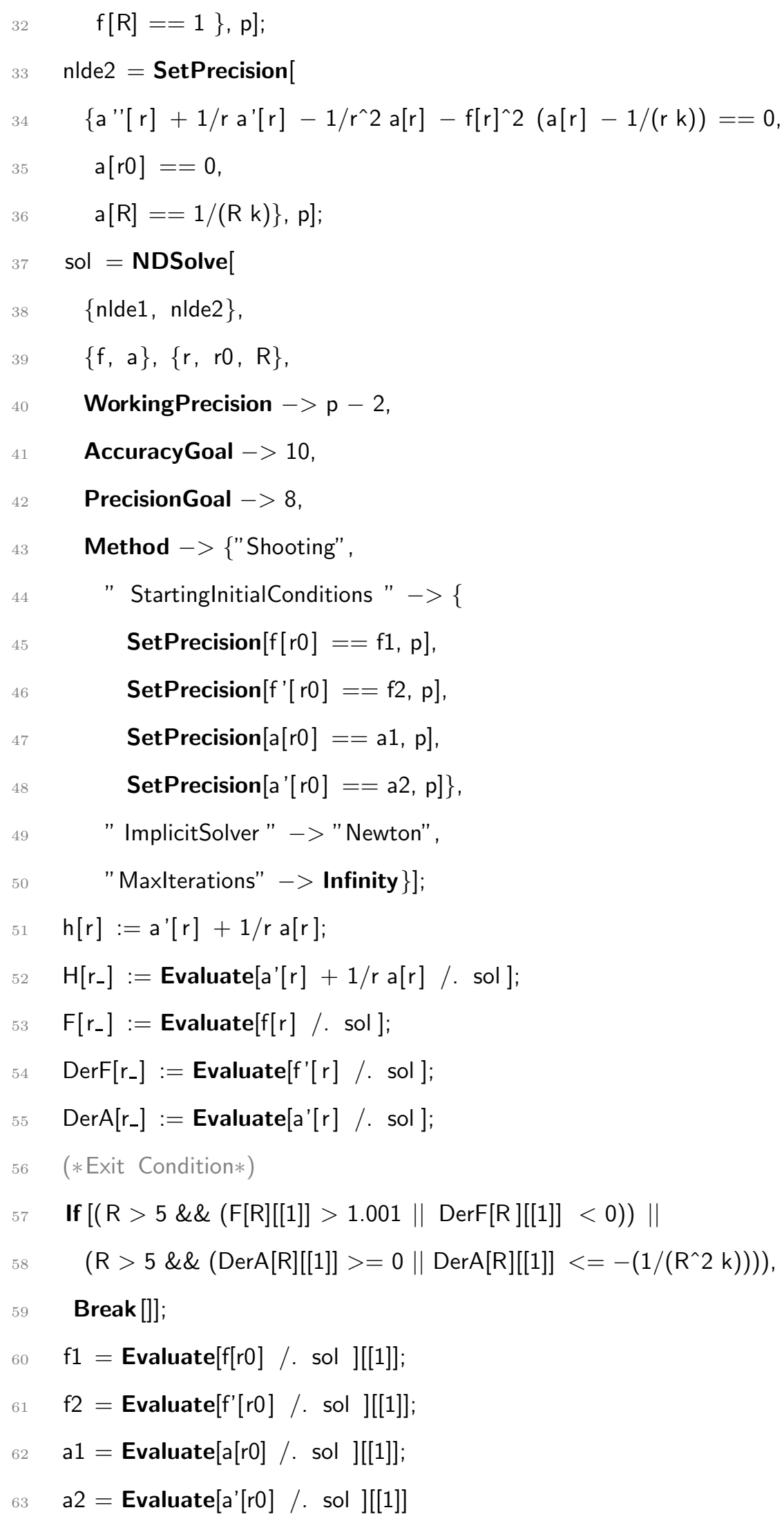




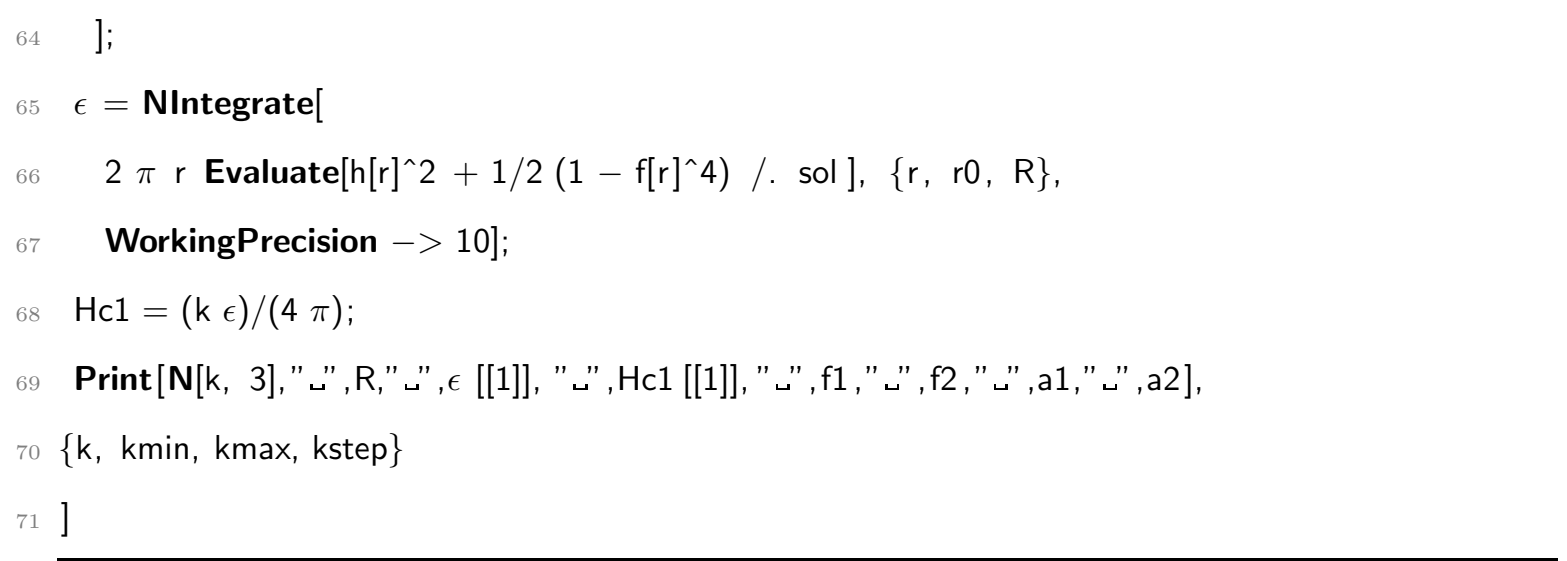

\section{D.2 Superheating Field Numerical Code}

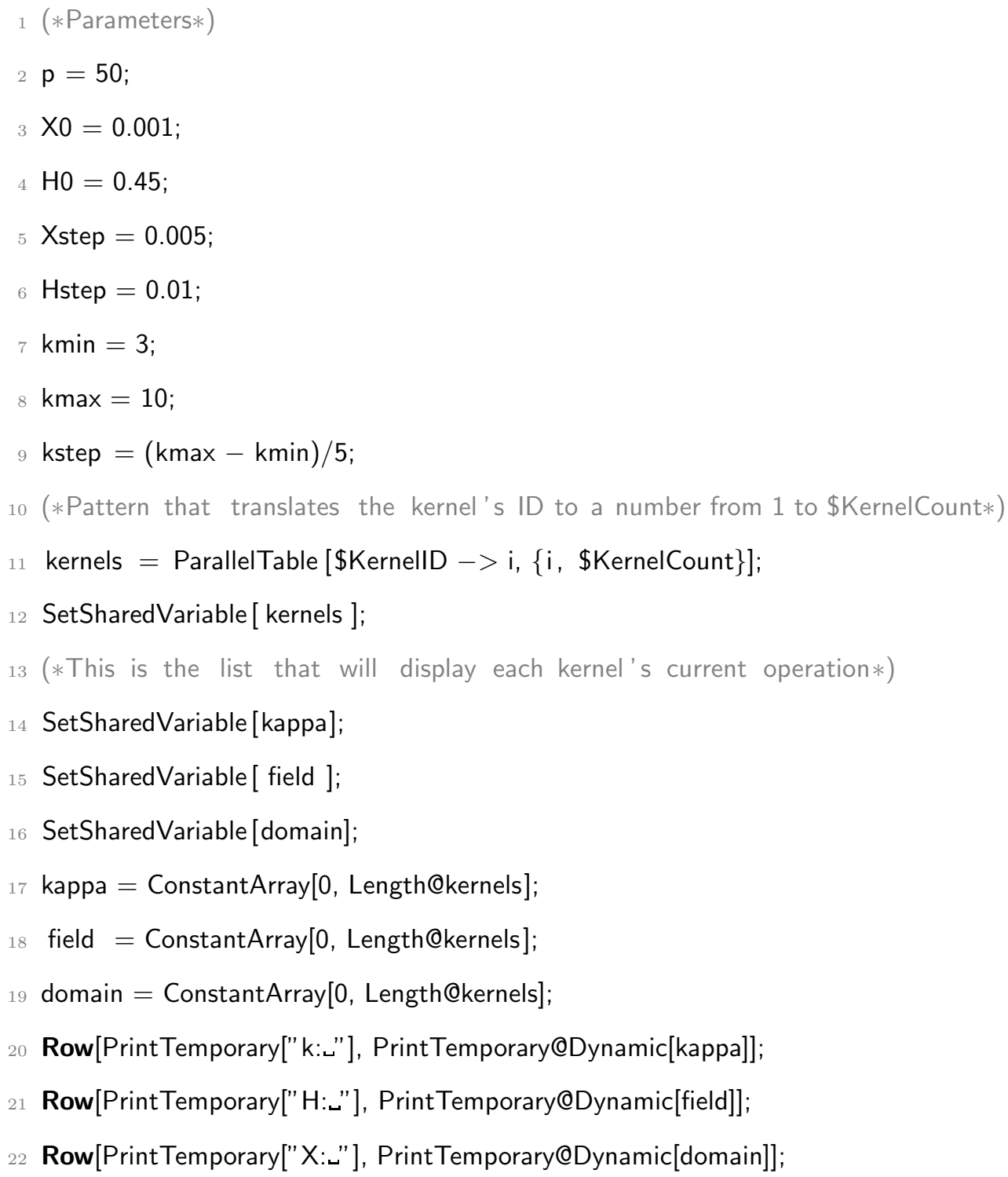




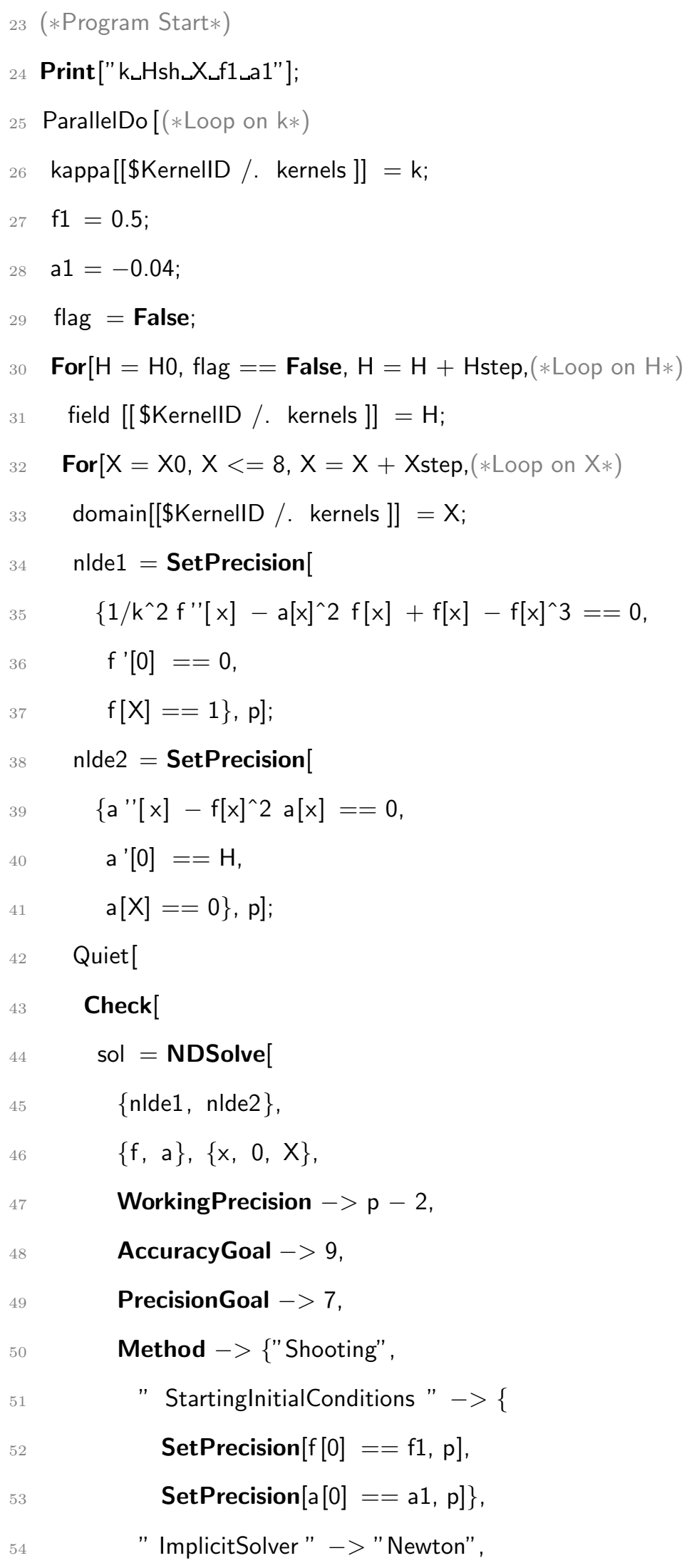




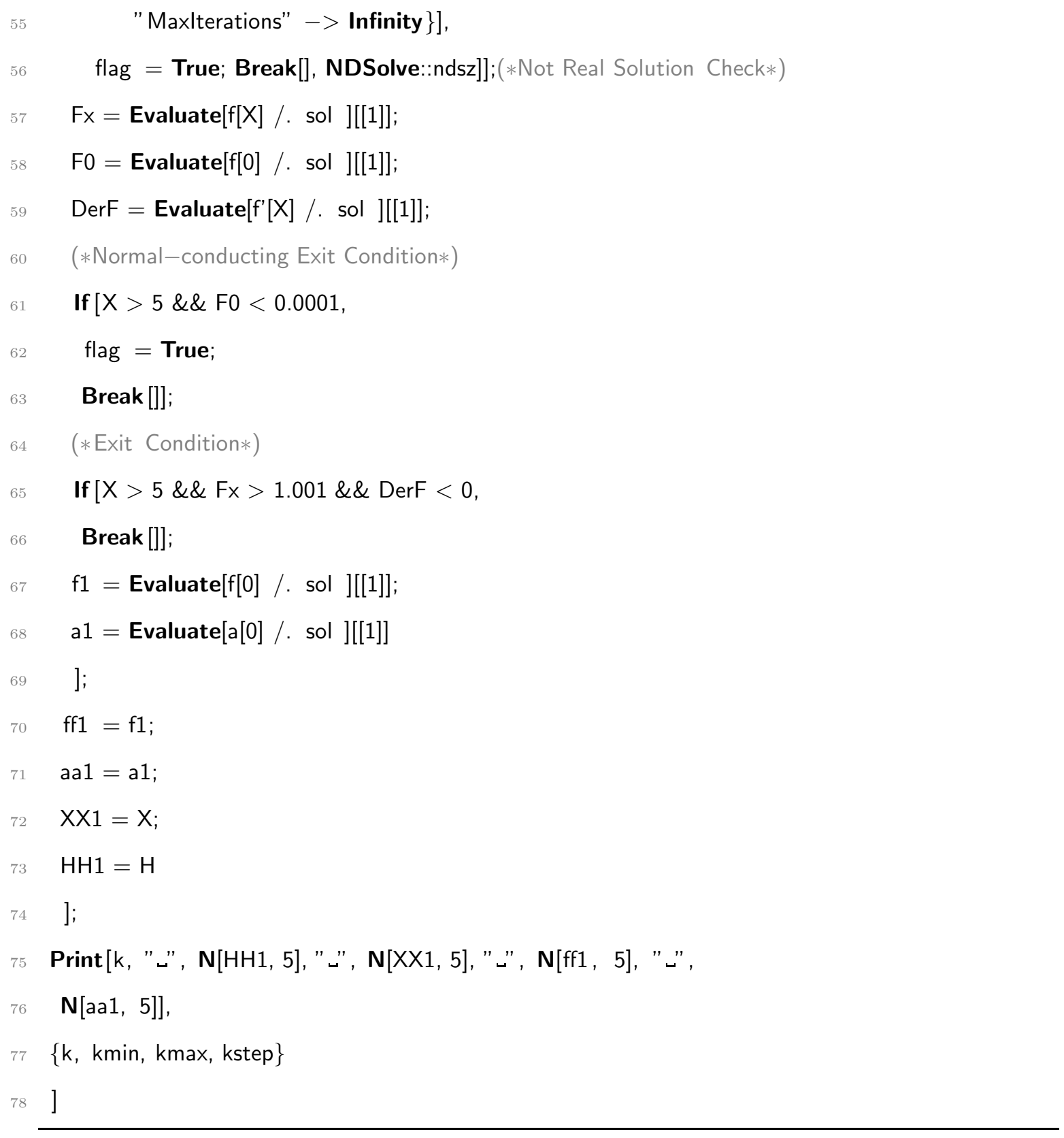




\section{BIBLIOGRAPHY}

[1] A. Yamamoto, ILC technical status, including cost and schedule (ILC Tokusui Workshop 2014), URL: https://agenda.linearcollider.org/event/6506/contributions/ 31389/attachments/25876/39733/ILC-ACc-Status-141216a.pdf

[2] R. P. Huebener and J. R. Clem, Magnetic flux structures in superconductors - a conference summary, Rev. Mod. Phys. 46 (1974), p. 409

[3] U. Essmann and H. Träuble, The direct observation of individual flux lines in type II superconductors, Physics Letters 24A (1967), p. 526

[4] H. F. Hess, R. B. Robinson, R. C. Dynes, J. J. M. Valles and J. V. Waszczak, Scanning-tunneling-microscope observation of the abrikosov flux lattice and the density of states near and inside a fluxoid, Phys. Rev. Lett. 62 (1989), p. 214

[5] M. R. Eskildsen, M. Kugler, S. Tanaka, J. Jun, S. M. Kazakov, J. Karpinski and $\varnothing$. Fischer, Vortex imaging in the $\pi$ band of magnesium diboride, Phys. Rev. Lett. 89 (2002), p. 187003

[6] P. E. Goa, H. Hauglin, M. Baziljevich, E. Il'yashenko, P. L. Gammel and T. H. Johansen, Real-time magneto-optical imaging of vortices in superconducting $\mathrm{NbSe}_{2}$, Supercond. Sci. Technol. 14 (2001), p. 729

[7] J. R. Delayen, RF properties of superconducting Pb electroplated onto $\mathrm{Cu}$, in Proc. of the 3rd Workshop on RF Superconductivity (1987), SRF87E02, p. 469

[8] A. Yamamoto, Advances in SCRF cavity performance in the technical design phase (2012),

URL: http://newsline.linearcollider.org/2012/03/29/

advances-in-scrf-cavity-performance-in-the-technical-design-phase/

[9] A. Septier and N. T. Viet, Microwave applications of superconducting materials, J. Phys. E: Sci. Instrum. 10 (1977), p. 1193

[10] M. Martinello, A. Grassellino, M. Checchin, A. Romanenko, O. Melnychuk, D. A. Sergatskov, S. Posen and J. F. Zasadzinski, Effect of interstitial impurities on the field dependent microwave surface resistance of niobium, Appl. Phys. Lett. 109 (2016), p. 062601

[11] A. Romanenko, A. Grassellino, F. Barkov, A. Suter, Z. Salman and T. Prokscha, Strong Meissner screening change in superconducting radio frequency cavities due to mild baking, Appl. Phys. Lett. 104 (2014), p. 072601

[12] A. A. Abrikosov, Fundamentals of the Theory of Metals (North Holland, 1988)

[13] H. Padamsee, J. Knobloch and T. Hays, RF Superconductivity for Accelerators (Wiley-VCH Verlag GmbH and Co., KGaA, Weinheim, 2008)

[14] H. Podlech, Superconducting versus normal conducting cavities, in Proc. of the CAS-CERN Accelerator School: High Power Hadron Machines (2011), p. 151 
[15] B. Aune, R. Bandelmann, D. Bloess, B. Bonin, A. Bosotti, M. Champion, C. Crawford, G. Deppe, B. Dwersteg, D. Edwards, H. T. Edwards, M. Ferrario, M. Fouaidy, P.-D. Gall, A. Gamp, A. Gössel, J. Graber, D. Hubert, M. Hüning, M. Juillard, T. Junquera, H. Kaiser, G. Kreps, M. Kuchnir, R. Lange, M. Leenen, M. Liepe, L. Lilje, A. Matheisen, W.-D. Möller, A. Mosnier, H. Padamsee, C. Pagani, M. Pekeler, H.-B. Peters, O. Peters, D. Proch, K. Rehlich, D. Reschke, H. Safa, T. Schilcher, P. Schmüser, J. Sekutowicz, S. Simrock, W. Singer, M. Tigner, D. Trines, K. Twarowski, G. Weichert, J. Weisend, J. Wojtkiewicz, S. Wolff and K. Zapfe, Superconducting TESLA cavities, Phys. Rev. ST Accel. Beams 3 (2000), p. 092001

[16] V. Shiltsev, A phenomenological cost model for high energy particle accelerators, J. Instrum. 9 (2014), p. T07002

[17] H. K. Onnes, Further experiments with liquid helium. C. On the change of electric resistance of pure metals at very low temperatures, etc. IV. The resistance of pure mercury at helium temperatures, Comm. Leiden 120b (1911)

[18] H. K. Onnes, Further experiments with liquid helium. D. On the change of electric resistance of pure metals at very low temperatures, etc. $V$. The disappearence of the resistance of mercury, Comm. Leiden 122b (1911)

[19] H. K. Onnes, Further experiments with liquid helium. G. On the change of electric resistance of pure metals at very low temperatures, etc. VI. On the sudden change in the rate at which the resistance of mercury disappears, Comm. Leiden 124c (1911)

[20] C. J. Gorter and H. Casimir, On supraconductivity I, Physica 1 (1934), p. 306

[21] F. London and H. London, The electromagnetic equations of the supraconductors, Proc. R. Soc. Lond. A 149 (1935), p. 71

[22] W. Meissner and R. Ochsenfeld, Ein neuer effekt bei eintritt der supraleitfähigkeit, Physica Naturwissenschaften 21 (1933), p. 787

[23] V. L. Ginzburg and L. D. Landau, On the theory of superconductivity, Zh. Eksp. Teor. Fiz. 20 (1950), p. 1064

[24] L. D. Landau, On the theory of phase transitions, Zh. Eksp. Teor. Fiz. 7 (1937), p. 19

[25] M. Tinkham, Introduction to Superconductivity (Dover Publications, 2004)

[26] A. A. Abrikosov, On the magnetic properties of superconductors of the second group, Zh. Eksp. Teor. Fiz. 32 (1957), p. 1442

[27] F. London, Superfluids, vol. 1 (John Wiley and Sons, New York, 1960)

[28] A. B. Pippard, An experimental and theoretical study of the relation between magnetic field and current in a superconductor, Proc. R. Soc. Lond. A 216 (1953), p. 547

[29] J. Bardeen, L. N. Cooper and J. R. Schrieffer, Theory of superconductivity, Phys. Rev. 108 (1957), p. 1175

[30] L. N. Cooper, Bound electron pairs in a degenerate fermi gas, Phys. Rev. 104 (1956), p. 1189 
[31] T. P. Sheehan, Rules for the energy gap and critical field of superconductors, Phys. Rev. 149 (1966), p. 368

[32] L. P. Gor'kov, Microscopic derivation of the Ginzburg-Landau equations in the theory of superconductivity, Zh. Eksp. Teor. Fiz. 36 (1959), p. 1918

[33] R. P. Huebener, Magnetic Flux Structures in Superconductors (Springer-Verlag, Berlin, Heidelberg, GmbH, 2001)

[34] L. V. Shubnikov, V. I. Khotkevich, Y. D. Shepelev and Y. N. Riabinin, Zh. Eksp. Teor. Fiz. 7 (1937), p. 221

[35] C. Caroli, P. G. de Gennes and J. Matricon, Bound fermion states on a vortex line in a type II superconductor, Phys. Lett. 9 (1964), p. 307

[36] J. Bardeen and M. J. Stephen, Theory on the motion of vortices in superconductors, Phys. Rev. 140 (1965), p. A1197

[37] T. Kubo, Flux trapping in superconducting accelerating cavities during cooling down with a spatial temperature gradient, Prog. Theor. Exp. Phys. (2016), p. 053G01

[38] A. Romanenko, A. Grassellino, O. Melnychuk and D. A. Sergatskov, Dependence of the residual surface resistance of superconducting radio frequency cavities on the cooling dynamics around Tc, J. Appl. Phys. 115 (2014), p. 184903

[39] M. Martinello, M. Checchin, A. Grassellino, A. C. Crawford, O. Melnychuk, A. Romanenko and D. A. Sergatskov, Magnetic flux studies in horizontally cooled elliptical superconducting cavities, J. Appl. Phys. 118 (2015), p. 044505

[40] S. Posen, M. Checchin, A. C. Crawford, A. Grassellino, M. Martinello, O. S. Melnychuk, A. Romanenko, D. A. Sergatskov and Y. Trenikhina, Efficient expulsion of magnetic flux in superconducting radiofrequency cavities for high $Q_{0}$ applications, J. Appl. Phys 119 (2016), p. 213903

[41] R. P. Huebener and A. Seher, Nernst effect and flux flow in superconductors. I. Niobium, Phys. Rev. 181 (1969), p. 701

[42] A. Gurevich and G. Ciovati, Effect of vortex hotspots on the radio-frequency surface resistance of superconductors, Phys. Rev. B 87 (2013), p. 054502

[43] G. Ciovati and A. Gurevich, Evidence of high-field radio-frequency hot spots due to trapped vortices in niobium cavities, Phys. Rev. ST Accel. Beams 11 (2008), p. 122001

[44] Y. B. Kim, C. F. Hempstead and A. R. Strnad, Flux-flow resistance in type-II superconductors, Phys. Rev. 139 (1965), p. A1163

[45] M. Tinkham, Viscous flow of flux in type-II superconductors, Phys. Rev. Lett. 13 (1964), p. 804

[46] L. P. Gor'kov and N. B. Kopnin, Vortex motion and resistivity of type-II superconductors in a magnetic field, Usp. Fiz. Nauk 116 (1975), p. 413

[47] P. G. de Gennes, Superconductivity of Metals and Alloys (Westview Press, 1999) 
[48] P. G. de Gennes and J. Matricon, Collective modes of vortex lines in superconductors of the second kind, Rev. Mod. Phys. 36 (1964), p. 45

[49] D. C. Mattis and J. Bardeen, Theory of the anomalous skin effect in normal and superconducting metals, Phys. Rev. 111 (1958), p. 412

[50] R. E. Glover and M. Tinkham, Conductivity of superconducting films for photon energies between 0.3 and $40 k T_{\mathrm{c}}$, Phys. Rev. 108 (1957), p. 234

[51] S. B. Nam, Theory of electromagnetic properties of strong-coupling and impure superconductors. II, Phys. Rev. 156 (1967), p. 487

[52] J. Halbritter, On surface resistance of superconductors, Z. Physik 266 (1974), p. 209

[53] J. P. Turneaure, J. Halbritter and H. A. Schwettman, The surface impedance of superconductors and normal conductors: The Mattis-Bardeen theory, J. Supercond. 4 (1991), p. 341

[54] A. Romanenko, F. Barkov, L. D. Cooley and A. Grassellino, Proximity breakdown of hydrides in superconducting niobium cavities, Supercond. Sci. Technol. 26 (2013), p. 035003

[55] C. Z. Antoine, How to achieve the best SRF performance: (Practical) Limitations and possible solutions, in Proc. of the CAS-CERN Accelerator School: Superconductivity for Accelerators (2013), p. 209

[56] M. Leitner, B. Bird, F. Casagrande, S. Chouhan, C. Compton, J. Crisp, K. Elliot, A. Facco, A. Fox, M. Hodek, M. Johnson, G. Kiupel, I. Malloch, D. Miller, S. Miller, D. Morris, D. Norton, R. Oweiss, J. Ozelis, J. Popielarski, L. Popielarski, A. Rauch, R. Rose, K. Saito, M. Shuptar, N. Usher, G. Velianoff, D. Victory, J. Wei, J. Whitaker, K. Witgen, T. Xu, Y. Xu, O. Yair and S. Zhao, The FRIB project at MSU, in Proc. of the 16th Workshop on RF Superconductivity (2013), MOIOA01, p. 1

[57] M. P. Kelly, J. D. Fuerst, S. Gerbick, M. Kedzie, K. W. Shepard, G. P. Zinkann and P. N. Ostroumov, Superconducting quarter-wave resonators for the ATLAS energy upgrade, in Proc. of the 24th Linear Accelerator Conference (2008), THP025, p. 836

[58] W. V. Delsolaro, S. Calatroni, B. Delaup, A. D’Elia, N. M. Jecklin, Y. Kadi, G. Keppel, D. Lespinasse, P. Maesen, I. Mondino, V. Palmieri, S. Stark, A. R. M. Sublet and M. Therasse, Nb sputtered quarter wave resonators for the HIE-ISOLDE, in Proc. of the 16th Workshop on RF Superconductivity (2013), WEIOA03, p. 767

[59] V. Yakovlev, T. Arkan, M. Awida, P. Berrutti, E. Borissov, A. Crawford, M. Foley, C. Ginsburg, A. Grassellino, C. Grimm, I. Gonin, S. Holmes, S. Kazakov, R. Kephart, T. Khabiboulline, V. Lebedev, A. Lunin, M. Merio, S. Nagaitsev, T. Nicol, Y. Orlov, D. Passarelli, T. Peterson, Y. Pischalnikov, O. Pronitchev, L. Ristori, A. Rowe, D. Sergatskov, N. Solyak, A. Sukhanov and I. Terechkine, Status of the SRF development for Project X, in Proc. of the 16th Workshop on RF Superconductivity (2013), MOP015, p. 117

[60] C. Darve, N. Elias, S. Molloy, P. Bosland, B. Renard, S. Bousson, G. Olivier, D. Reynet and J. P. Thermeau, The ESS superconducting RF cavity and cryomodule cryogenic processes, Physics Procedia 67 (2015), p. 1123 
[61] D. Boussard, E. Chiaveri, E. Haebel, H. Kindermann, R. Losito, S. Marque, V. Rödel and M. Stirbet, The LHC superconducting cavities, in Proc. of the 18th Particle Accelerator Conference (1999), p. 946

[62] e. C. Adolphsen, The International Linear Collider Technical Design Report, Accelerator in the Technical Design Phase - Volume 3.I (2013)

[63] B. W. J. McNeil and N. R. Thompson, X-ray free-electron lasers, Nat. Photon 4 (2010), p. 814

[64] M. Waldrop, The big guns, Nature 505 (2014), p. 604

[65] P. Bishop, M. Checchin, H. Conklin, A. Crawford, E. Daly, K. Davis, M. Drury, R. Eichhorn, J. Fischer, F. Furuta, G. M. Ge, D. Gonnella, A. Grassellino, C. Grimm, T. Gruber, D. Hall, A. Hocker, G. Hoffstaetter, J. Kaufman, G. Kulina, M. Liepe, J. Maniscalco, M. Martinello, O. Melnychuk, T. OConnel, J. Ozelis, A. D. Palczewski, P. Quigley, C. Reece, A. Romanenko, M. Ross, A. Rowe, D. Sabol, J. Sears, D. A. Sergatskov, W. Soyars, R. Stanek, V. Veshcherevich, R. Wang and G. Wu, LCLS-II SRF cavity processing protocol development and baseline cavity performance demonstration, in Proc. of the 17th International Conference on RF Superconductivity (2015), MOPB033, p. 159

[66] I. S. Park, Y. U. Sohn, J. H. Choi, M. S. Hong, H. C. Kwon, W. H. Hwang, D. T. Kim, Y. C. Kim, C. W. Chung, J. Y. Hung, I. S. Ko, S. H. Kim, S. U. Kim and S. S. Song, Fabbrication of Ichiro nine-cell cavities in PAL for STF of KEK, in Proc. of the 22nd Particle Accelerator Conference (2007), WEPMN060, p. 2173

[67] M. Checchin and M. Martinello, Analytic solution of the electromagnetic eigenvalues problem in a cylindrical resonator (2016),

URL: https://arxiv.org/pdf/1610.02083v1.pdf

[68] T. P. Wangler, RF Linear Accelerators (Wiley-VCH Verlag GmbH and Co., KGaA, Weinheim, 2008)

[69] J. Knobloch, H. Muller and H. Padamsee, Design of a high speed, high resolution thermometry system for $1.5 \mathrm{GHz}$ superconducting radio frequency cavities, Rev. Sci. Instrum. 65 (1994), p. 3521

[70] G. Ciovati, Effect of low-temperature baking on the radio-frequency properties of niobium superconducting cavities for particle accelerators, J. Appl. Phys. 96 (2004), p. 1591

[71] A. Romanenko, C. J. Edwardson, P. G. Coleman and P. J. Simpson, The effect of vacancies on the microwave surface resistance of niobium revealed by positron annihilation Spectroscopy, App. Phys. Lett. 102 (2013), p. 232601

[72] A. Grassellino, A. Romanenko, D. A. Sergatskov, O. Melnychuk, Y. Trenikhina, A. C. Crawford, A. Rowe, M. Wong, T. Khabiboulline and F. Barkov, Nitrogen and argon doping of niobium for superconducting radio frequency cavities: $A$ pathway to highly efficient accelerating structures, Supercond. Sci. Tech. 26 (2013), p. 102001

[73] E. H. Brandt, The flux-line lattice in superconductors, Rep. Prog. Phys. 58 (1995), p. 1465 
[74] A. M. Campbell and J. E. Evetts, Flux vortices and transport currents in type II superconductors, Adv. Phys. 21 (1972), p. 199

[75] M. Martinello, M. Checchin, A. Grassellino, O. Melnychuk, S. Posen, A. Romanenko, D. A. Sergatskov and J. F. Zasadzinski, Trapped flux surface resistance analysis for different surface treatments, in Proc. of the 17th International Conference on RF Superconductivity (2015), MOPB015, p. 115

[76] M. Martinello, M. Checchin, A. Grassellino, O. Melnychuk, S. Posen, A. Romanenko, D. A. Sergatskov and J. F. Zasadzinski, Tailoring surface impurity content to maximize Q-factors of superconducting resonators, in Proc. of the 7th International Particle Accelerator Conference (2016), WEPMR003, p. 2258

[77] J. I. Gittleman and B. Rosenblum, Radio-frequency resistance in the mixed state for subcritical currents, Phys. Rev. Lett. 16 (1966), p. 734

[78] M. W. Coffey and J. R. Clem, Vortex-motion dissipation in high- $T_{\mathrm{c}}$ superconductors at microwave frequencies, Phys. Rev. Lett. 67 (1991), p. 386

[79] R. Marcon, R. Fastampa, M. Giura and E. Silva, Vortex-motion dissipation in high- $T_{\mathrm{c}}$ superconductors at microwave frequencies, Phys. Rev. B 43 (1991), p. 2940

[80] M. Rabinowitz, Analysis of a critical loss in a superconductor, J. Appl. Phys. 42 (1971), p. 88

[81] M. Rabinowitz, Frequency dependence os superconducting cavity $Q$ and magnetic breakdown fields, Appl. Phys. Lett. 19 (1971), p. 73

[82] E. B. Sonin, A. K. Tagantsev and K. B. Traito, Two-mode electrodynamics of superconductors in the mixed state, Phys. Rev. B 43 (1992), p. 5830

[83] T. Matsushita, Flux Pinning in Superconductors (Springer-Verlag, Berlin, Heidelberg, 2014)

[84] L. Embon, Y. Anahory, A. Suhov, D. Halbertal, J. Cuppens, A. Yakovenko, A. Uri, Y. Myasoedov, M. L. Rappaport, M. E. Huber, A. Gurevich and E. Zeldov, Probing dynamics and pinning of single vortices in superconductors at nanometer scales, Sci. Rep. 5 (2015), p. 7598

[85] B. W. Maxfield and W. L. McLean, Superconducting penetration depth of niobium, Phys. Rev. 139 (1965), p. A1515

[86] S. J. Williamson and L. E. Valby, Nonlocal characteristic of the bulk upper critical field of niobium, Phys. Rev. Lett. 24 (1970), p. 1061

[87] N. W. Ashcroft and N. D. Mermin, Solid State Physics (Harcourt College Publishers, 1976)

[88] D. Janjušević, M. S. Grbić, M. Požek, A. Dulčić, D. Paar, B. Nebendahl and T. Wagner, Microwave response of thin niobium films under perpendicular static magnetic fields, Phys. Rev. B 74 (2006), p. 104501

[89] L. H. Allen and J. H. Claassen, Technique for measuring the elementary pinning force in thin films, Phys. Rev. B 39 (1989), p. 2054 
[90] G. S. Park, C. E. Cunningham, B. Cabrera and M. E. Huber, Vortex pinning force in a supereonducting niobium strip, Phys. Rev. Lett. 68 (1992), p. 1920

[91] M. Golosovsky, D. Davidov, E. Farber, T. Tsach and M. Schieber, Microwave transmission and harmonic generation in granular high- $T_{\mathrm{c}}$, superconducting films: Evidence for viscous flux motion and weak links, Phys. Rev. B 43 (1991), p. 43

[92] M. Golosovsky, M. Tsindlekht and D. Davidov, High-frequency vortex dynamics in $\mathrm{YBa}_{2} \mathrm{Cu}_{3} \mathrm{O}_{7}$, Supercond. Sci. Technol. 9 (1996), p. 1

[93] M. M. Asim and S. K. Hasanain, Modified flux flow in polycrystalline YBCO: Temperature and current dependence, Supercond. Sci. Technol. 9 (1996), p. 461

[94] C. Benvenuti, S. Calatroni, I. E. Campisi, P. Darriulat, M. A. Peck, R. Russo and A.-M. Valente, Study of the surface resistance of superconducting niobium films at $1.5 \mathrm{GHz}$, Physica C 316 (1999), p. 153

[95] D. L. Hall, J. J. Kaufman, M. Liepe and J. T. Maniscalco, RF measurements on high performance $\mathrm{Nb}_{3} \mathrm{Sn}$ Cavities, in Proc. of the 7th International Particle Accelerator Conference (2016), WEPMR024, p. 2320

[96] A. Godeke, A review of the properties of $\mathrm{Nb}_{3} \mathrm{Sn}$ and their variation with A15 composition, morphology and strain state, Supercond. Sci. Technol. 19 (2006), p. R68

[97] R. M. Scalan, W. A. Fietz and E. F. Koch, Flux pinning centers in superconducting $\mathrm{Nb}_{3} \mathrm{Sn}$, J. Appl. Phys. 46 (1975), p. 2244

[98] B. J. Shaw, Grain size and film thickness of $\mathrm{Nb}_{3} \mathrm{Sn}$ formed by solid-state diffusion in the range 650-800 C, J. Appl. Phys. 47 (1976), p. 2147

[99] H. Padamsee, RF Superconductivity: Volume II: Science, Technology and Applications (Wiley-VCH Verlag GmbH and Co., KGaA, Weinheim, 2009)

[100] A. Gurevich, Multiscale mechanisms of SRF breakdown, Physica C 441 (2006), p. 38

[101] T. Kubo, Magnetic field enhancement at a pit on the surface of a superconducting accelerating cavity, Prog. Theor. Exp. Phys. (2015), p. 073 G01

[102] T. Kubo, Models of the magnetic field enhancement at pits, in Proc. of the 16th International Conference on RF Superconductivity (2013), TUP008, p. 443

[103] P. Tholfsen and H. Meissner, Cylindrically symmetric solutions of the GinzburgLandau equations, Phys. Rev. 169 (1968), p. 306

[104] J. L. Harden and V. Arp, The lower critical field in the Ginzburg-Landau theory of superconductivitys, Cryogenics 3 (1963), p. 105

[105] J. Matricon and D. Saint-James, Superheating field in superconductors, Phys. Lett. 24 (1967), p. 241

[106] A. J. Dolgert, S. J. Di Bartolo and A. T. Dorsey, Superheating fields of superconductors: Asymptotic analysis and numerical results, Phys. Rev. B 53 (1996), p. 5650 
[107] M. K. Transtrum, G. Caletani and J. P. Sethna, Superheating field of superconductors within Ginzburg-Landau theory, Phys. Rev. B 83 (2011), p. 094505

[108] S. Casalbuoni, E. A. Knabbe, J. Kötzler, L. Lilje, L. von Sawilski, P. Schmüser and B. Steffen, Surface superconductivity in niobium for superconducting RF cavities, Nucl. Instr. Meth. Phys. Res. A 538 (2005), p. 45

[109] Orsay Group on Superconductivity, Field dependence of the superconducting properties induced by proximity effects: breakdown fields, Phys. Kondens. Materie 6 (1967), p. 307

[110] C. P. Bean and J. D. Livingston, Surface barrier in type-II superconductors, Supercond. Sci. Technol. 12 (1964), p. 14

[111] A. Gurevich, Maximum screening fields of superconducting multilayer structures, AIP Advances 5 (2015), p. 017112

[112] T. Kubo, Y. Iwashita and T. Saeki, Radio-frequency electromagnetic field and vortex penetration in multilayered superconductors, Appl. Phys. Lett. 104 (2014), p. 032603

[113] T. Kubo, Multilayer coating for high gradients (2016),

URL: https://arxiv.org/abs/1607.01495v2.pdf

[114] S. Posen, M. K. Transtrum, G. Caletani, M. U. Liepe and J. P. Sethna, Shielding superconductors with thin films as applied to rf cavities for particle accelerators, Phys. Rev. Applied 4 (2015), p. 044019

[115] T. Kubo, Superconducting nano-layer coating without insulator, in Proc. of the 27th Linear Accelerator Conference (2014), THPP074, p. 1026

[116] M. Checchin, M. Martinello, A. Grassellino, A. Romanenko, S. Posen and J. F. Zasadzinski, Ultimate gradient limitation in niobium superconducting accelerating cavities, in Proc. of the 7th International Particle Accelerator Conference (2016), WEPMR002, p. 2254

[117] M. Checchin, M. Martinello, A. Grassellino, A. Romanenko, S. Posen and J. F. Zasadzinski, Enhancement of the Accelerating Gradient in Superconducting Microwave Resonators, in Proc. of the 28th Linear Accelerator Conference (2016), TUPRL024

[118] M. Hein, High-Temperature Superconductor Thin Films at Microwave Frequencies (Springer-Verlag, Berlin, Heidelberg, 1999)

[119] J. Knobloch and H. Padamsee, Flux trapping in niobium cavities during breakdown events, in Proc. of the 8th Workshop on RF Superconductivity (1997), B06, p. 337

[120] I. M. Terechkine, T. N. Khabiboulline and D. A. Sergatskov, Performance degradation of a superconducting cavity quenching in magnetic field, in Proc. of the 16th International Conference on RF Superconductivity (2013), THP047, p. 1113

[121] T. N. Khabiboulline, J. Ozelis, D. A. Sergatskov and I. M. Terechkine, Superconducting cavity quenching in the presence of magnetic field, FNAL Note TD-11-020 (2011) 
[122] T. N. Khabiboulline, D. A. Sergatskov and I. M. Terechkine, SSR1 cavity quenching in the presence of magnetic field, FNAL Note TD-12-007 (2012)

[123] T. N. Khabiboulline, D. A. Sergatskov and I. M. Terechkine, $650 \mathrm{MHz}$ elliptical cavity performance degradation induced by magnetic field of a test coil, FNAL Note TD-13-002 (2013)

[124] M. Checchin, M. Martinello, A. Romanenko, A. Grassellino, D. A. Sergatskov, S. Posen, O. Melnychuk and J. F. Zasadzinski, Quench-induced degradation of the quality factor in superconducting resonators, Phys. Rev. Applied 5 (2016), p. 044019

[125] M. Checchin, M. Martinello, A. Romanenko, A. Grassellino, O. Melnychuk and D. A. Sergatskov, Origin of trapped flux caused by quench in superconducting niobium cavities, in Proc. of the 6th International Particle Accelerator Conference (2015), WEPT021, p. 3309

[126] M. Checchin, Nature of the quality factor degradation in SRF cavities due to quench, in Proc. of the 17 th International Conference on RF Superconductivity (2015), MOBA05, p. 41

[127] T. E. Faber and A. B. Pippard, Kinetics of the phase transition in superconductors, Progr. Low. Temp. Phys. 1 (1955), p. 159

[128] S. Antipov, E. Efimenko, A. Romanenko and D. A. Sergatskov, Time-resolved measurements of high field quench in SRF cavities, in Proc. of the 16th International Conference on RF Superconductivity (2013), TUP112, p. 743 